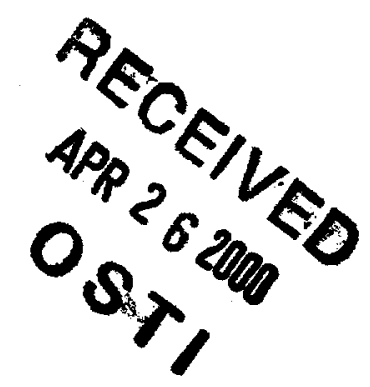

Development of Highly Magnetostrictive Composites for Applicationsin Magnetomechanical Torque Sensors

by

Chen, Yonghua

PHD Thesis submitted to Iowa state University

Ames Laboratory, U.S. DOE

Iowa State University

Ames, Iowa 50011

Date 1999

PREPARED FOR THE U.S. DEPARTMENT OF ENERGY

UNDER CONTRACT NO. W-7405-Eng-82 . 


\section{This report was prepared as an account of work sponsored by an agency of the
United States Government. Neither the United States Government nor any
agency thereof, nor any of their employees, makes any warranty, express or
implied, or assumes any legal liability or responsibility for the accuracy,
completeness or usefulness of any information, apparatus, product, or process
disclosed, or represents that its use would not infringe privately owned rights.
Reference herein to any specific commercial product, process, or service by trade
name, trademark, manufacturer, or otherwise, does not necessarily constitute or
imply its endorsement, recommendation, or favoring by the United States
Government or any agency thereof. The views and opinions of authors expressed
herein do not necessarily state or reflect those of the United States Government
or any agency thereof.}

This report has been reproduced directly from the best available copy.

AVAILABILITY:

To DOE and DOE contractors: Office of Scientific and Technical Information

P.O. Box 62

Oak Ridge, TN 37831

prices available from: $(615) 576-8401$

FTS: $626-8401$

To the public:

National Technical Information Service

U.S. Department of Commerce

5285 Port Royal Road

Springfield, VA 22161 


\section{DISCLAIMER}

Portions of this document may be illegible in electronic image products. Images are produced from the best available original document. 


\section{TABLE OF CONTENTS}

ABSTRACT $\quad$ vii

1. GENERAL INTRODUCTION

1.1 Background 1

1.2 Scope of work 4

2. FUNDAMENTALS OF MAGNETISM

2.1 Concepts of magnetism $\quad 6$

2.1.1 Magnetic field $\mathbf{H}$ and magnetic induction $\mathbf{B} \quad 6$

$\begin{array}{ll}\text { 2.1.2 Magnetic moment } \mathbf{m} \text { and magnetization } \mathbf{M} & 7\end{array}$

$\begin{array}{ll}\text { 2.1.3 Demagnetizing field } & 8\end{array}$

2.2 Magnetic materials $\quad 9$

2.2.1 Magnetic hysteresis loop 9

$\begin{array}{ll}\text { 2.2.2 Thermal effect on magnetic materials } & 11\end{array}$

$\begin{array}{ll}\text { 2.2.3 Magnetostriction } & 11\end{array}$

$\begin{array}{ll}\text { 2.2.4 Anisotropy } & 12\end{array}$

$\begin{array}{ll}\text { 2.3 Domains and domain dynamics } & 12\end{array}$

$\begin{array}{ll}\text { 2.3.1 Domains and domain wall } & 12\end{array}$

$\begin{array}{ll}\text { 2.3.2 Domain wall pinning } & 15\end{array}$

2.3.3 Domain processes 15

$\begin{array}{ll}\text { 2.4 Magnetic measurements } & 17\end{array}$

3. MAGNETOSTRICTION AND MAGNETOSTRICTIVE MATERIALS 19

3.1 Introduction 19 
3.2 Magnetostrictive rare earth- $\mathrm{Fe}_{2}$ compounds

3.3 Magnetostrictive ferrites

3.4 Composite materials

4. MAGNETOMECHANICAL EFFECT AND ITS APPLICATIONS

4.1 Villari effect - magnetomechanical effect under uniaxial stress

4.2 Inverse Wiedemann effect - magnetomechanical effect under torsional stress

4.2.1 Direct and inverse Wiedemann effect

4.2.2 Stresses distribution under torque

4.2.3 Torque dependence of the magnetization

5.1 Preparation of Terfenol-based composites

5.2 Preparation of Co ferrite and magnetic properties measurement

5.3 Magnetostriction measurement

5.4 Magnetic torque sensor test bed

$5.5 \mathrm{Fe}, \mathrm{Ni}$, Co rods and magnetomechanical effects measurement

5.6 Co-ferrite rings and measurement of magnetomechanical effects

5.7 Measurement of temperature dependence of magnetomechanical effects

\section{RESULTS AND DISCUSSION}

6.1 Magnetostriction of Terfenol-based composites

6.1.1 Terfenol + soda lime glass

6.1.2 Terfenol + resin 
6.1.4 Terfenol + metal $\left(\mathrm{Fe}, \mathrm{Cu}, \mathrm{Al}, \mathrm{CeFe}_{2}\right)+\mathrm{NaPO}_{3}$ glass $\quad 50$

6.1.5 Terfenol $+\mathrm{NaPO}_{3}$ glass 51

6.1.6 Summary of magnetostriction of Terfenol-based composites

6.2 Magnetostriction of Co ferrite and its composites $\quad 52$

6.3 Comparison of magnetostriction in Terfenol-based and Co ferrite composites 56

6.4 Magnetomechanical effect under torsional strain of iron, cobalt and nickel metals

6.4.1 $\sigma / \tau-\mathrm{H}$ process measurements

6.4.2 $\mathrm{H}-\sigma / \tau$ process measurements

6.4.3 Discussion on the magnetomechanical effect under torsional stress

6.4.4 Summary of magnetomechanical effects in metal rods

6.5 Magnetomechanical effects under torsional stress of Co-ferrite composites

6.5.1 Major loop

6.5.2 Minor loops

6.5.3 Homogeneity

6.6 Temperature dependence of magnetomechanical effects under torsional stress 78

6.6.1 Results

6.6.2 Discussion

6.7 Summary of metal-bonded Co ferrite composites

\section{MODEL OF MAGNETOSTRICTION AND MAGNETOMECHANICAL EFFECTS}

7.1 Magnetostriction model

7.1.1 Previous study

7.1.2 Effect of elastic modulus on composite magnetostrictive materials 
7.1.2.1 Uniform strain case

7.1.2.2 Uniform stress case

7.1.2.3 Magnetostriction limits

7.1.2.4 Comparison of experimental and modeled results

7.1.2.5 Summary

7.2 Magnetomechanical effect model

7.2.1 Effective field due to stress and stress dependent anhysteretic curve

7,2.2 Law of approach

7.3 Extension of the model under uniaxial stress to torsional stresses

7.3.1 Effective field due to torque

100

7.3.2 Law of approach

101

7.3.3 Rotation of magnetization with respect to torque

101

7.3.4 Modeling procedure

103

7.3.5 Comparison of experimental and modeled results

104

7.3.6 Summary

110

8. CONCLUSIONS

111

APPENDIX

114

REFERENCES

157

ACKNOWLEDGEMENTS 


\begin{abstract}
The objective of this work was to investigate and develop a magnetomechanical material with high magnetomechanical response and low hysteresis. This material will be used in electronic torque sensors for advanced steering systems in automobiles which will replace the costly and fuel inefficient hydraulic steering systems currently in use. Magnetostriction and the magnetomechanical effect under torsional stress of magnetostrictive composites have been investigated in the present study.

The effect of the matrix material on the magnetostriction of composites containing highly magnetostrictive particles was first studied. Experimental results showed that the elastic modulus of the matrix is an important factor determining the bulk magnetostriction of the composite. For a series of composites with the same volume fraction of Terfenol-D particles but different matrix materials, the bulk magnetostriction was found to increase systematically with decreasing elastic modulus of the matrix. A model theory for the magnetostriction of such composites was developed, based on two limiting assumptions: uniform strain or uniform stress inside the composite. The modeled results were in agreement with the experimental results.
\end{abstract}

A magnetic torque sensor test bed was developed as part of the present investigation. This instrumentation was used to make the magnetomechanical measurements under torsional stress. After investigating both the $\mathrm{H}-\sigma$ processes and $\sigma-\mathrm{H}$ processes, the change of magnetization as a function of applied torque at remanence state exhibited substantially linear behavior. The derivative of circumferential surface magnetic field with respect to torque for a $\mathrm{Ni}$ rod was $18.9 \mathrm{AN}^{-1} \mathrm{~m}^{-2}$ in the torque range of $\pm 5 \mathrm{~N} \cdot \mathrm{m}$. It 
was shown that a high piezomagnetic coefficient, together with a high saturation magnetostriction are two "figures of merit" for choosing magnetostrictive sensing materials.

A new class of magnetomechanical sensor materials, Co-ferrite $\left(\mathrm{CoO} \cdot \mathrm{Fe}_{2} \mathrm{O}_{3}\right)$ and metal-bonded $(\mathrm{Ag} / \mathrm{Ni} / \mathrm{Co})$ Co-ferrite composites, has been found for this application. These materials exhibited magnetostriction in excess of $200 \mathrm{ppm}$ and a $d_{33}$ coefficient, $1.3 \times 10^{-9} \mathrm{~A}^{-1} \mathrm{~m}$. Selected compositions were fabricated into test samples in the form of rings which were brazed onto stainless steel through-shafts. The sensitivity of the surface axial magnetic field to applied torque was observed for a demonstration sample to be as high as $65 \mathrm{AN}^{-1} \mathrm{~m}^{-2}$ in the torque range of $\pm 10 \mathrm{~N} \cdot \mathrm{m}$.

The temperature dependence of the magnetomechanical sensitivity and hysteresis were measured over the range -37 to $90^{\circ} \mathrm{C}$. Both decreased as the temperature increased throughout the entire range. The magnetomechanical hysteresis became negligible at temperatures higher than $60^{\circ} \mathrm{C}$, above which it gave a linear magnetic field change in response to applied torque. These temperature dependencies were explained by the changes of magnetostriction, anisotropy, spontaneous magnetization and pinning of domain walls caused by the availability of increased thermal energy.

The magnetomechanical effect under torque was modeled with an extension of the existing uniaxial model of the magnetomechanical effect. The modeled results show similar behavior to the experimental results and give quantitatively realistic values of sensitivity and hysteresis. 


\section{GENERAL INTRODUCTION}

\subsection{Background}

Since Joule discovered magnetostriction in 1842 [1], there has been continuing interest in the field of magnetoelasticity, often for the development of magnetostrictive devices. Magnetostriction may be defined as the change in shape and size of any substance due to a change in magnetic order. Magnetoelasticity is the science dealing with the coupling between the magnetic and elastic properties of matter [2]. Magnetostriction originates from the magnetoelastic coupling. Conversely, almost any stress applied to the ferromagnetic material will produce a change of its magnetization due to the magnetoelastic coupling. This phenomenon is the magnetomechanical effect (sometimes erroneously called inverse magnetostriction).

Magnetostriction can play an important role in all magnetic materials because the magnetization of a ferromagnetic material, even paramagnetic materials is in nearly all cases accompanied by changes in dimensions and vice versa. Sometimes, magnetostriction is detrimental and needs to be avoided or minimized because it tends to hinder the magnetization processes and to increase the coercivity and hysteresis loss. For instance, the magnetostriction in magnetic recording heads, and electric transformers should be eliminated if possible. On the other hand, highly magnetostrictive materials are good candidates for sensors and actuators due to their high sensitivity of magnetization to stress which can be used to develop non-contact sensors. The first technical applications of magnetostriction, the oscillator, the torquemeter [3] appeared before 1930. The interest 
in applied magnetoelasticity rose dramatically in 1970 s due to the discovery of new giant magnetostrictive materials, Terfenol-D $\left(\mathrm{Tb}_{0.3} \mathrm{Dy}_{0.7} \mathrm{Fe}_{2}\right)$ [4]. The goal of the research which formed this dissertation was to develop highly magnetostrictive composites for applications in magnetomechanical sensors, particularly for torque sensor applications in automobiles.

Since the 1970 s when the first electronic sensors were incorporated into automobiles, the number of microelectronic sensors has increased in the past twenty years from typically 20 per car in 1980 to 70 per car in 1990 and in over 100 per car in 1996 [5]. There is a well-established need for torque sensors for automobiles. Such sensors will be used for electronic control of steering and control of drive shafts. It is known that replacement of hydraulic steering systems with "electronic" systems can achieve a 5\% improvement in fuel economy because the hydraulic systems continuously draw power, whereas the electronic systems are low power and draw power as needed. The 5\% fuel efficiency improvement implies that 3.7 billions gallons of gasoline can be saved for US passengers cars alone by using the new magneto-electronic steering system. In addition, electronic systems are generally less massive.

A magnetomechanical sensor has been considered a promising candidate for steering sensor applications. The prime advantage of a magnetic torque sensor is that change of magnetization can be conveniently sensed by a Hall effect probe or other magnetic measurement method, such magnetoresistive (MR) and giant magnetoresistive (GMR) sensors without contacting the shaft subjected torque. 
Magnetic torque sensors can be divided into two categories. The first measures small angles or displacements resulting from torque, using a magnetic circuit [6]. This requires considerable space and is mechanically complicated. The second employs the effect of torque on magnetization in magnetic materials. In this case, highly magnetostrictive materials are required. In earlier studies $[7,8]$, magnetic torque sensing was investigated directly on iron/steel shafts using an external magnetic yoke carrying a drive coil and pickup coil. This kind of sensor was restricted to small stress and magnetic induction due to the low magnetostriction characteristics of iron. The accuracy and the dynamic range were further limited due to the magnetic inhomogeneities and induced eddy currents in the shaft.

Amorphous alloys have received much attention as a material for constituting force transducers [10] and torque sensors [11-13]. This series of materials are usually FeSi-B based Metglas ${ }^{\mathrm{TM}}$ alloys. They are made by cooling the molten alloy at a rate of $10^{6}$ ${ }^{\circ} \mathrm{C} / \mathrm{s}$. It is a general-purpose soft magnetic material (coercivity less than $10 \mathrm{~A} / \mathrm{m}$ ) for core loss applications. Amorphous ribbon transducers exhibit wide dynamic range and good linearity. However they need to be attached to the shaft to sense the applied torque. The amorphous magnetic torque transducers sense the change of permeability with excitation coils (or permanent magnets) under different torques. The sensor configuration and also detected signal can be complicated because of the relationship between applied field and magnetization. Garshelis [14] proposed a simple magnetized ring sensor, which did not require external field excitation. The sensor was operated in a remanent state. The surface magnetic field from such a sensor measured by a Hall probe was found to be proportional 
to applied torque. However, the maraging steel was used in that study did not exhibit a high magnetostriction (less than 30ppm).

\subsection{Scope of work}

The requirements for the magnetostrictive torque sensor for automobile applications are:- sufficiently high magnetoelastic response, temperature stability to allow it to operate over a temperature range of $-40^{\circ} \mathrm{C}$ to $85^{\circ} \mathrm{C}$, and adequate mechanical strength with the lowest possible cost. The choice of the sensor material is a critical factor in magnetic torque sensor applications, which determines the sensitivity, mechanical performance and cost as well. In order to develop materials to achieve these requirements, the research was composed of six parts:

1. Investigate magnetostriction of composite magnetostrictive materials and the effect of matrix material on magnetic and mechanical properties. Screen proper materials for magnetomechanical torque sensors. The measurement and sample preparation are much simpler and less expensive for magnetostrictive studies than those of the magnetomechanical effect. Two series of candidate materials are, Terfenol/Terfenolbased composites, Co ferrite \& metal-bonded composites.

2. Design and build a magnetic torque sensor test bed. This equipment was used to measure the magnetomechanical effect of materials under torsional stress.

3. Investigate the magnetomechanical effect of conventional magnetic materials ( $\mathrm{Fe}, \mathrm{Co}, \mathrm{Ni}$ ) under torsional stress. The purpose is to relate magnetostriction and the magnetomechanical effect of materials and to explore proper measuring mode(s) for 
magnetomechanical torque sensing. The conventional materials were chosen because their magnetostrictions have been fully investigated and the metal samples are easier and less expensive to prepare.

4. Design a prototype torque sensor using the candidate magnetostrictive materials with guidance of the results of part 1 and the recommendation of part 3. Conduct the magnetomechanical effect measurement under torsional. stress of the prototype sensors and evaluate materials performance.

5. Investigate the temperature dependence of the performance of the best prototype sensor selected in part 4.

6. Model magnetostriction, magnetomechanical effects and explore their relationship. The models are done interactively with the experimental results and used to assist selection of materials and design of the sensors. Eventually the computational models developed here will be used for computer aided design (CAD) of sensors. 


\section{FUNDAMETALS OF MAGNETISM}

\subsection{Concepts of magnetism}

\subsubsection{Magnetic field $\mathbf{H}$ and magnetic induction $\mathbf{B}$}

When a magnetic field is generated in a volume of space, there is a change in energy of that volume. A magnetic field $\mathbf{H}$ is produced according to Maxwell's equations whenever there is electrical current. Conventionally, a permanent magnet can also produce a magnetic field. In this case, in fact, there are the orbital motions and spins of electrons inside which generate the magnetic field. Therefore, broadly, it could be stated that magnetic field originates from any motion of electric charge. The unit of magnetic field is Ampere per meter. For standard purpose, all magnitudes in this dissertation will be in SI units (meter, kilogram, second and Ampere).

On the other hand, magnetic induction $\mathbf{B}$ is the response of a medium when a magnetic field is present in the medium. Magnetic induction is sometimes called the flux density. The unit of magnetic induction is Tesla. The relation between magnetic field and magnetic induction is determined by the permeability of the medium, $\mu$.

$$
\mathbf{B}=\mu \mathbf{H}
$$

where the permeability is not necessarily a constant, or even a single value for ferromagnetic materials. However, the permeability of free space, $\mu_{0}$, is a constant and its value is $4 \pi \times 10^{-7}$ Henry/meter. 


\subsubsection{Magnetic moment $\mathbf{m}$ and magnetization $\mathbf{M}$}

Magnetic moment is the most elementary unit of magnetism. There are two widely used models, equivalent current loop model and dipole model, which both are merely mathematical approximations, as shown in Fig. 2.1. In the current loop, the magnitude of the magnetic moment is the product of the current $i$ and the area of the loop

A. The direction of the magnetic moment is determined by the right-hand rule. Microscopically, the current can be described as the motion of electrons and spins of unpaired electrons. The magnitude of the magnetic moment in the dipole model equals the product of the pole strength $p$ and the distance between poles $l$ although the single magnetic pole (the monopole) has never been discovered. The direction of the magnetic moment in this case points from the south (-p) pole to the north pole $(+p)$.

Magnetization is defined as the vector sum of magnetic moment per unit volume of a solid.

$$
\mathbf{M}=\mathbf{m} / \mathbf{V}
$$
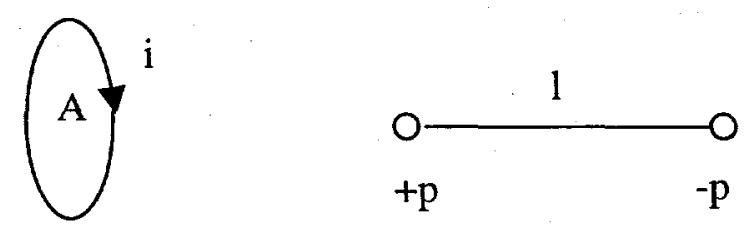

Fig. 2.1 Two basic mathematical entities: the current loop and dipole 
In bulk materials, the magnetizations of magnetic materials depend on the responses of these materials to the magnetic field. The relation is described by another quantity, susceptibility $\chi$.

$$
\chi=\mathbf{M} / \mathbf{H}
$$

The unit of magnetization is $\mathrm{A} / \mathrm{m}$.

The magnetic induction $\mathbf{B}$ is composed of two components: one from the magnetic field and the other from the magnetization. Now, we can relate the three important quantities, magnetic field $\mathbf{H}$, magnetic induction $\mathbf{B}$ and magnetization $\mathbf{M}$, using the following equation

$$
\mathbf{B}=\mu_{0}(\mathbf{H}+\mathbf{M})
$$

\subsubsection{Demagnetizing field}

When we consider a magnetized material of finite dimension in the dipole model, both ends of the material are two magnetic poles, which themselves cause a magnetic field in the material. The direction of this field is opposite to the direction of the magnetization. The field is then called the demagnetizing field $\mathbf{H}_{\mathrm{d}}$. The demagnetizing field depends on the magnetization of the material and demagnetizing factor, $\mathrm{N}_{\mathrm{d}}$, which is determined by the sample geometry.

$$
\mathbf{H}_{\mathrm{d}}=-\mathrm{N}_{\mathrm{d}} \mathbf{M}
$$

The demagnetizing field plays an important role in magnetic materials, particularly in high permeability materials $(\mathbf{M}>>\mathbf{H})$ and permanent magnets. 


\subsection{Magnetic materials}

Magnetic materials are mainly classified into three groups, diamagnets, paramagnets, and ferromagnets [15], according to their responses to a magnetic field, e.g. susceptibility. Diamagnets are those materials for which susceptibilities are negative and $\approx 10^{-5}$. Their magnetic responses oppose the applied magnetic field. Paramagnets are those materials for, which $\chi$ are positive and the value are $\approx 10^{-3}-10^{-5}$. The third group includes most widely recognized magnetic materials, which are called ferromagnets. Their susceptibility is positive and typically has value $50-10,000$ [15]. Other groups, such as ferrimagnetic materials and antiferromagnetic materials sometimes are classified into ferromagnet since they have strong response to a magnetic field. Antiferromagnetic materials have strong exchange like ferromagnets even though their susceptibilities are small like paramagnets.

In this dissertation, the phrase "magnetic materials" will refer to ferromagnetic materials, including ferrimagnetic and antiferromagnetic materials, while other materials will be called non-magnetic materials.

\subsubsection{Magnetic hysteresis loop}

The response of a magnetic material, which is represented by the magnetization $\mathbf{M}$ or magnetic induction $\mathbf{B}$ not only depends on the applied field $\mathbf{H}$ but also the magnetization history of the sample. The response of a magnetic material $\mathbf{B}$ usually depends non-linearly on the change of applied magnetic field $\mathbf{H}$ and then is called hysteresis. The most common way to describe the magnetic properties of a material is the 
hysteresis loop, which is a plot of $\mathbf{M}$ or $\mathbf{B}$ versus $\mathbf{H}$. One typical hysteresis loop, as shown in Fig.2-2 presents most characteristics of the materials, including saturation magnetization $\mathbf{M}_{\mathbf{s}}$, coercivity $\mathbf{H}_{\mathrm{c}}$, remanence $\mathbf{M}_{\mathrm{r}}$, permeability $\mu$ and initial permeability $\mu_{\mathrm{in}}$.

Generally, magnetic materials are simply classified into two groups according to their coercivities: soft magnets and hard magnets (also sometimes called permanent magnets). Broadly speaking, magnetic materials with coercivities above $10 \mathrm{kA} / \mathrm{m}$ are hard magnets and soft magnets are those with coercivities below $1 \mathrm{kA} / \mathrm{m}$. However, as more magnetic materials, better properties and more broad applications of magnetic materials are discovered and developed, this classification is inadequate, and even unclear.

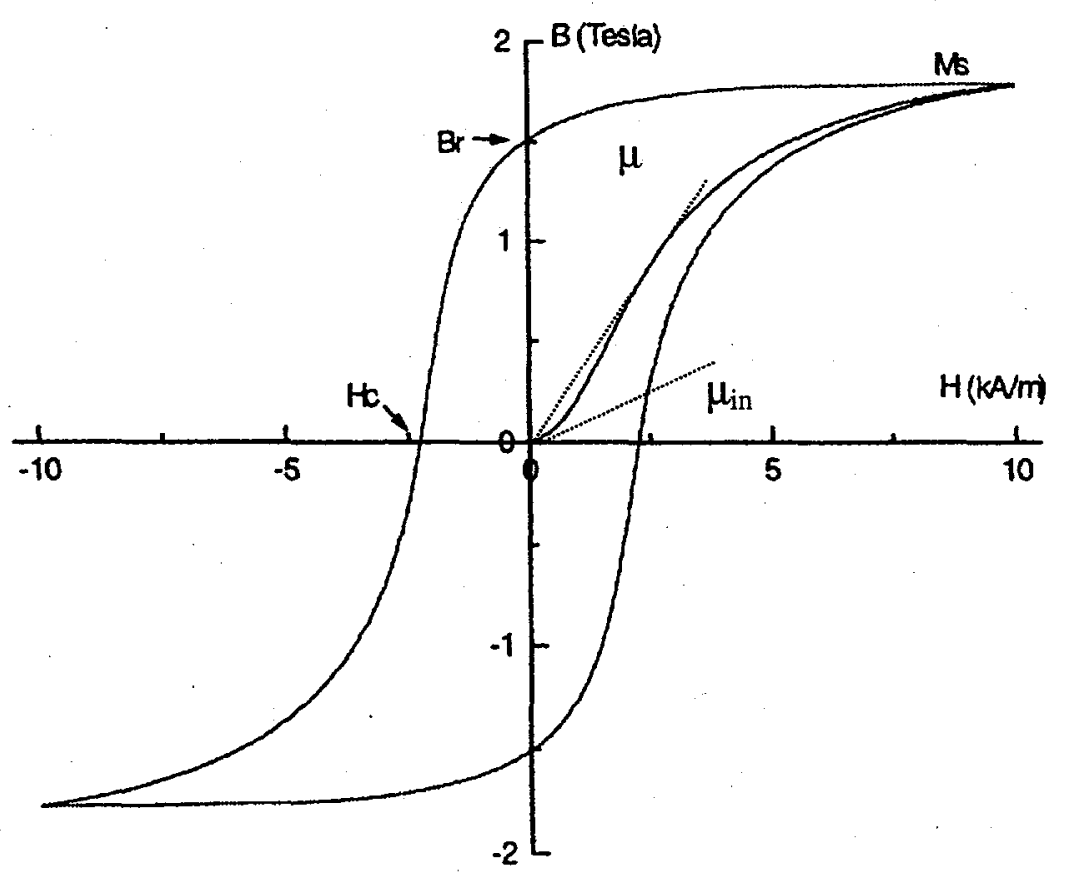

Fig 2-2 A typical hysteresis loop of a ferromagnetic material 


\subsubsection{Thermal effect on magnetic materials}

All ferromagnetic materials have the maximum spontaneous magnetization with the domains at $0 \mathrm{~K}$ and this decreases as the temperature increases. The decrease of spontaneous magnetization is due to magnetic moment precession caused by thermal energy. Furthermore, the materials become paramagnetic when heated to a characteristic temperature, which is called the Curie temperature. At the transition temperature, the saturation magnetization of the material suddenly drops. The reason is that the thermal energy of the magnetic moment reaches a critical value at Curie temperature which destroys the magnetic order in the ferromagnetic materials.

\subsubsection{Magnetostriction}

The magnetization of a ferromagnetic material is usually accompanied by changes in dimensions. The resulting strain is called magnetostriction. The magnetostriction originates from magnetoelastic coupling. The magnetoelastic energy associated with magnetostrictive strain can be expressed by [16]:

$$
\mathrm{E}=\frac{3}{2} \lambda_{\mathrm{s}} \sigma \sin ^{2} \theta
$$

where $\lambda_{s}$ is the saturation magnetostriction, $\sigma$ is the stress and, and $\theta$ is the angle between magnetization and stress. This energy predicts that the magnetization of a material with positive magnetostriction prefers to align along the tensile stress axis while the magnetization of a material with negative magnetostriction prefers the compressive stress axis. Hence, magnetostriction and its coupling with the stress field in magnetic materials has a strong effect on magnetic properties of magnetic materials. 


\subsubsection{Anisotropy}

Almost all materials are anisotropic to some degree, which implies that the magnetic properties are not identical in all direction. In crystalline materials, the preferred directions of magnetization are called magnetic easy axes, or preferred axes. The anisotropic energy is defined as the value of energy required to turn the magnetization from a preferred "easy" direction into a so-called "hard" direction. A oneconstant equation is used as a first approximation of the anisotropy of cubic materials [15]

$$
\mathrm{E}_{\mathrm{a}}=\mathrm{K}_{1}\left(\cos ^{2} \theta_{1} \cos ^{2} \theta_{2}+\cos ^{2} \theta_{2} \cos ^{2} \theta_{3}+\cos ^{2} \theta_{1} \cos ^{2} \theta_{3}\right)
$$

where $\theta_{1}, \theta_{2}, \theta_{3}$ are angles which the magnetization makes relative to the three crystal axes.

The anisotropy of hexagonal materials is given by one constant approximation [15]

$$
\mathrm{E}_{\mathrm{a}}=\mathrm{K}_{\mathrm{ul}} \sin ^{2} \phi
$$

where $\phi$ is the angle of magnetization with respect to the unique axis.

The anisotropy energy is strain dependent so that magnetostriction and anisotropy of materials are related.

\subsection{Domains and domain dynamics}

\subsubsection{Domains and domain wall}

The theory of static domains and domain walls can be explained by thermodynamic energy minimization in magnetic materials. 
According to theory, an individual atom has a magnetic moment due to the spins of unpaired electrons in ferromagnets, and also in paramagnets. However, in ferromagnets, there are strong mutual interactions among the magnetic moments, which make the behavior of ferromagnetic materials so different from non-magnetic materials. According to Heisenberg's expression, the exchange interaction between spin $S_{i}$ and $S_{j}$ is given by

$$
E_{i j}=-2 J S_{i} \cdot S_{j}=-2 J S^{2} \cos \varphi
$$

Macroscopically (domain scale), the exchange interaction can be approximately considered as a Weiss mean field.

$$
\mathrm{H}_{\mathrm{e}}=\alpha \mathrm{M}
$$

where, $\alpha$ is the Weiss mean field constant and $\mathbf{M}$ is the bulk magnetization. The exchange energy forces the magnetic moments to align parallel locally to decrease the energy of a ferromagnetic material, where $\alpha$ is positive. Another energy that should be taken into account is magnetostatic energy, which is expressed by

$$
E=-H_{d} M=\mu_{0} N_{d} M^{2} / 2
$$

The magnetostatic energy attempts to keep the net bulk magnetization zero also due to energy minimization. The breakup of magnetization into localized regions, providing for flux closure at the ends of specimen, reduces the magnetostatic energy. Those localized regions are called domains. Within each domain, the magnetic moments of all atoms are aligned parallel. In demagnetized states, the domains are aligned randomly if the 
materials are isotropic and are aligned along their crystallographic magnetic easy axes if the materials are anisotropic.

We may ask how the magnetic moments behave in the domain boundary. In general, there is a transition region in which the magnetic moments change directions gradually from one domain to the other domain. This region is known as the domain wall, or Bloch wall. If a specimen is in the form of thin film, the Bloch wall would have its magnetization normal to the plane of the material, which causes a large demagnetizing energy. The Neel wall, in which the moments rotate within the plane of the specimen, results in a lower overall energy for domain walls in thin films.

The domain wall thickness is determined by two energies, exchange energy between neighboring moments and anisotropy energy. The exchange energy tends to keep the angle between moments or neighboring atoms small because the exchange energy is smaller when neighboring moments are aligned closer to parallel. The anisotropy, on the other hand, tends to make the wall thinner since the anisotropy energy is smaller when all moments align along easy axes. The domain wall energy is considered as the sum of exchange energy and anisotropy energy of the moments in a unit area of domain wall.

The domain size is the result of competition between the magnetostatic energy and the domain wall energy. The magnetostatic energy tends to decrease the size of domains while the domain wall energy tends to increase the size. Typically, there are $10^{12}$ to $10^{18}$ atomic moments in each domain [15]. All these analyses above are based on 
thermodynamic equilibrium. The real situation is more complicated if the kinetics of the process are considered.

\subsubsection{Domain wall pinning}

The domain and domain wall structures in magnetic materials not only depend on the material's composition, which determines the exchange energy and the domain wall energy, but also depend on the metallurgy and treatment of the materials, which determines, stress distribution, dislocations, voids and second phases. Since these defects result in local energy minima when domain walls intersect them, an extra energy is needed for domain walls to move. This is called the domain wall pinning energy. In other words, it is the magnetoelastic coupling between the structural defects and the domain walls that affects the motion of the domain wall, therefore controls the magnetic properties, such as permeability, coercivity.

\subsubsection{Domain processes}

In the demagnetized state, magnetic domains are randomly arranged while magnetic moments within domains are all aligned collinear to each other along one of the crystallographic easy axes. Under the action of an applied magnetic field (other "effective fields" will be discussed later), magnetic domain processes occur. The driving force comes from the magnetic field energy,

$$
\mathrm{E}=-\mu_{0} \mathbf{m} \cdot \mathbf{H}
$$


because the energy minimum occurs when all moments are aligned parallel with the applied field. Basically, there are two domain processes: domain wall motion and domain rotation. The domain wall motion process causes the growth of domains which are aligned with respect to the field together with a reduction in size of domains which are aligned opposing the field. The domain rotation process occurs when the domains which are not in the direction of the applied magnetic field, overcome the anisotropy energy and rotate towards the direction of the field. Both processes are either reversible or irreversible. In fact, the domain wall motion is also caused by magnetic moment rotations, where the rotating moments are located within the domain walls and each moment rotates through a small angle.

Generally, the number of moments within domain walls is much less than the number of moments within domains. At low applied field, domain wall motion usually dominates the magnetization processes unless there are very strong pinning sites and domain wall energies are too high to be moved, e.g. very hard materials. Wall motion incorporates two distinct effects: domain wall bowing and translation. Domain wall bowing occurs when the domain wall energy is low and the pinning strength is strong. Domain wall bowing is a reversible process at low field, in which a domain wall under the action of a magnetic field behaves like an elastic membrane. When the field is removed the wall returns to its original position. Domain wall bowing becomes irreversible once the domain wall is sufficiently deformed that the expansion continues without further increase of field. Wall translation is usually irreversible and occurs at moderate field strength unless the material is nearly free of defects, which implies few domain wall 
pinning sites. In the domain wall translation process, the domain wall breaks away from its current pinning site and becomes pinned by another pinning site. At higher applied magnetic field, the domain wall bowing and translation processes might occur together.

At moderate field strength, domain rotation becomes significant because the magnetic field energy can be high enough to overcome the anisotropy energy. When the angular displacement of the domain's magnetization is slight, it is reversible. If the moments rotate from their original easy axes to the easy axis closest to the field direction, it is irreversible.

At some point, all moments are aligned along their closest easy axes within all crystalline grains. In other words, single domains are formed at high field in all single grains in a polycrystalline material and a single domain is obtained in a single crystal material. At yet higher applied field, coherent rotation occurs. The magnetic moments are gradually rotated from preferred easy axes towards the field direction. This process is also reversible. Finally at very high fields there is a reversible change in which the magnetic moments within the sample, which is already a single domain, are aligned more closely with the field direction. This occurs because the individual magnetic moments precess about the field direction due to thermal energy. As the field strength increases the angle of precession is reduced.

\subsection{Magnetic measurements}

There are many means of measuring the magnetic field, magnetic induction and magnetization. The two common methods most used in magnetic measurements are the 
induction method and the Hall effect method. The principle of the induction method is Faraday's law. It states that the e.m.f. induced in a circuit equals the rate of change of magnetic flux passing through the circuit.

$$
\mathrm{V}=-\mathrm{Nd} \Phi / \mathrm{dt}=-\mathrm{NAdB} / \mathrm{dt}
$$

where $A$ is the area of the circuit and $N$ is the number of turns of the circuit and $\mathbf{B}, \Phi$ are flux density and flux, respectively. This method can only measure the rate of change of magnetic induction not the absolute value.

The Hall effect can be briefly described as follows: when a magnetic field is applied to a conducting material carrying an electric current, there is a transverse Lorentz force on the charge carriers. Under the Lorentz force, the direction of motion of the charge carriers deviates from the original direction of the current and a potential difference is generated between the two transverse sides. The potential is proportional to the magnetic field strength $\mathbf{H}$. Therefore, this potential can be used to sense the magnetic field strength. The advantage of this method is that it can measure static magnetic field.

Magnetostriction of materials is usually measured using strain gauges. When a thin metallic wire is stretched, its length is increased and its cross-section is reduced; both effects tend to increase its electrical resistance. When the wires are bonded well on the surface of sample, the change of resistance is proportional to deformation of the sample. This is the basic idea of strain gage. 


\section{MAGNETOSTRICTION AND MAGNETOSTRICTIVE MATERIALS}

There are several excellent books which described magnetostriction $[2,15-19]$. Sometimes, the distinction between magnetostriction and the magnetomechanical effect (inverse magnetostriction) is not made. In the present work, the term magnetostriction is strictly reserved to describe the change in dimensions of a material as its magnetization changes. The term magnetomechanical effect refers to the magnetization change caused by stress.

\subsection{Introduction}

Magnetic material's behavior is so different from non-magnetic materials because of the strong exchange interaction among the moments, spin-orbit coupling. The exchange interaction is further dependent on the distance between magnetic moments. That is the origin of magnetoelastic coupling: magnetic states of a material, especially a ferromagnetic material, affect the dimension (magnetostriction), and its magnetic properties are influenced by the applied and internal stresses (magnetomechanical effect, Villari effect [2]).

Phenomenologically, magnetostriction can be divided into two types: spontaneous magnetostriction which arises from the ordering of magnetic moments into domains at the Curie temperature, and field-induced magnetostriction arising from the reorientation of domains. Field-induced magnetostriction is the more interesting feature of magnetostrictive materials for applications. 
Magnetic anisotropy plays an important role in the magnetostriction of materials. A crystal deforms spontaneously if to do so lowers the anisotropy energy. On the other hand, anisotropy may be an obstacle to the application of a magnetostrictive material, if the anisotropy is too high. In that case, much higher magnetic field is needed to produce the saturation magnetostriction.

The linear magnetostriction is simply defined as $\lambda$, the fractional change in length under the influence of an applied magnetic field

$$
\lambda=\frac{\mathrm{dl}}{1}
$$

While the magnetostriction arises from magnetic anisotropy energy, the magnetostriction itself is anisotropic and is not identical in all directions. The anisotropic magnetostriction in a single crystal cubic material is given by [15]

$$
\lambda_{s}=\frac{3}{2} \lambda_{100}\left(\alpha_{1}^{2} \beta_{1}^{2}+\alpha_{2}^{2} \beta_{2}^{2}+\alpha_{3}^{2} \beta_{3}^{2}-\frac{1}{3}\right)+3 \lambda_{111}\left(\alpha_{1} \alpha_{2} \beta_{1} \beta_{2}+\alpha_{2} \alpha_{3} \beta_{2} \beta_{3}\right)
$$

where $\alpha$ and $\beta$ are the direction cosines of magnetization and strain measurement relative to the crystal axes, respectively. $\lambda_{100}$ is the saturation magnetostriction along the $\langle 100\rangle$ direction and $\lambda_{111}$ is the saturation magnetostriction along the $<111>$ direction. The saturation magnetostriction of a polycrystalline material, an assembly of domains, can then be calculated by averaging the effects. In a totally randomly oriented polycrystalline cubic material (i.e. no texture), the saturation magnetostriction is given by

$$
\lambda_{s}=\frac{2}{5} \lambda_{100}+\frac{3}{5} \lambda_{111}
$$

The saturation magnetostriction measured at an angle to the field direction is expressed as 


$$
\lambda_{s}(\theta)=\frac{3}{2} \lambda_{s}\left(\cos ^{2} \theta-\frac{1}{3}\right)
$$

Magnetostrictions and anisotropies of some conventional magnetic materials and typical magnetostrictive materials are shown in Table 3-1. Besides linear magnetostriction, there are two other magnetostrictions: transverse magnetostriction and volume magnetostriction. At low field, the volume of materials keep constant, then transverse magnetostriction is equal to half of longitude magnetostriction.

$$
\lambda_{t}=-\lambda / 2
$$

Volume magnetostriction, the change in volume caused by the application of a magnetic field is usually much smaller than the change in length and often occurs at high field.

Table 3-1 magnetostriction, anisotropy and Curie temperature of selected materials

\begin{tabular}{lccccc}
\hline \hline Material & $\lambda_{100}\left(10^{-6}\right)$ & $\lambda_{111}\left(10^{-6}\right)$ & $\lambda s\left(10^{-6}\right)$ & $\mathrm{K}_{1}\left(10^{3} \mathrm{~J} / \mathrm{m}^{3}\right)$ & $\mathrm{T}_{\mathrm{c}}(\mathrm{K})$ \\
\hline Iron [15] & 21 & -21 & & 47 & 1043 \\
Nickel [15] & -46 & -24 & -33 & -59 & 631 \\
$\mathrm{CoO} \cdot \mathrm{Fe}_{2} \mathrm{O}_{3}[16]$ & -920 & 180 & -260 & $60^{*}$ & 793 \\
$\mathrm{TbFe}_{2}[4]$ & & & 1753 & $>1000$ & 700 \\
$\mathrm{SmFe}_{2}[4]$ & & & -1560 & & 676 \\
$\mathrm{Terfenol}$ [-D [15] & 90 & 1640 & $\sim 1000$ & 60 & $650-700$ \\
\hline \hline
\end{tabular}

* This value is very sensitive to the exact stoichiometry, $\mathrm{Co} / \mathrm{Fe}$ ratio and heat treatments. 


\subsection{Magnetostrictive rare earth- $\mathrm{Fe}_{2}$ compounds}

Although the phenomenon of magnetostriction has been known for more than one and half centuries, its wide applications were not realized until the huge magnetostrictions $(\sim 1 \%)$ of $\mathrm{Tb}$ and Dy was discovered at low temperature in 1963 [4]. Since then, the research activity in magnetostriction has been dramatically stimulated $[4,18,21-26]$.

There existed two questions before the application of these magnetostrictive properties could be achieved at room temperature. First of all, almost all rare earths possess Curie temperatures lower than room temperature. In order to increase the Curie temperatures, highly magnetostrictive rare earths, $\mathrm{Tb}$ and $\mathrm{Dy}$ were combined with the magnetic transition metals, $\mathrm{Ni}, \mathrm{Co}$ and $\mathrm{Fe}$. Some of those compounds possess high Curie temperatures, up to $1000 \mathrm{~K}$ and perform moderate magnetostriction at room temperature. In 19.71, Laves phase $\mathrm{RFe}_{2}\left(\mathrm{TbFe}_{2}, \mathrm{SmFe}_{2}\right)$ compounds were found to exhibit high magnetostrictions ( $>1000 \mathrm{ppm})$ at room temperature [4].

However, the anisotropies of those compounds are extremely large, the high magnetostriction can only obtained at very high magnetic field $(8 \mathrm{MA} / \mathrm{m})$, two orders of magnitude higher than that of $\mathrm{Ni}, \mathrm{Fe}$. At first thought, high magnetostriction and low anisotropy are incompatible. But if two rare-earth compounds have the opposite sign of magnetic anisotropy and the same sign of magnetostriction, the compound combining then will have very interesting properties. Clark at the Naval Ordnance Laboratory, discovered that $\mathrm{Tb}$ and Dy make such a perfect pair [21]. The compound is $\mathrm{Tb}_{0.3} \mathrm{Dy}_{0.7} \mathrm{Fe}_{2}$, which is named as Terfenol-D ("Ter" derived from terbium, "fe" to represent the iron, "nol" for the Naval Ordnance Laboratory and D to denote the dysprosium). 
However, Terfenol-D is quite expensive and susceptible to corrosion because of the rare-earth elements $\mathrm{Tb}$ and $\mathrm{Dy}$. It is also mechanically brittle. So, a Terfenol-based composite was considered as a promising solution to improve the mechanical properties and to lower the cost $[27,28]$. For the same purpose, $\mathrm{SmFe}_{2}$ and its composites have been studied as well [29].

\subsection{Magnetostrictive ferrites}

Ferrites are ferrimagnetic materials, which consist of oxides where small magnetic ions are localized in crystallographic sites embedded with the lattice formed by oxygen ions. The general theory of ferrimagnetism was developed by Neel. One of the most important features is the magnetization of the sublattices having opposite signs due to their negative magnetic coupling. Furthermore, the temperature dependencies of magnetic moments on the two sublattices are different. So, the magnetization may even change sign with changing temperature. The temperature, at which the sublattice magnetizations just cancel, is called $\mathrm{T}_{\text {comp. }}$

Ferrites are classified into two main groups: soft ferrites and hard ferrites. Soft ferrites exhibit high permeability, low coercivity and high resistivity suitable for high frequency applications, although their saturation magnetizations are somewhat low $(0.30-$ $0.5 \mathrm{MA} / \mathrm{m}$ ). Soft ferrites are usually in cubic structures (spinel or garnet). Hard ferrites $\left(\mathrm{BaO} \cdot 6 \mathrm{Fe}_{2} \mathrm{O}_{3}\right.$ or $\left.\mathrm{SrO} \cdot 6 \mathrm{Fe}_{2} \mathrm{O}_{3}\right)$ are highly anisotropic materials with hexagonal structures.

The magnetostrictions of ferrites are also strongly temperature dependent. Among those materials, Co ferrite $\left(\mathrm{CoO} \cdot \mathrm{Fe}_{2} \mathrm{O}_{3}\right)$ has high magnetostriction at room temperature. 
Co ferrite has a cubic spinel structure. The spinel ferrites generally have the chemical formula $\mathrm{MO} \cdot \mathrm{Fe}_{2} \mathrm{O}_{3}$, where $\mathrm{M}$ could be a metal such as nickel, iron, cobalt, titanium, chromium, manganese, copper or zinc. The $\mathrm{MO} \cdot \mathrm{Fe}_{2} \mathrm{O}_{3}$ unit cell consists of 56 atoms. 32 oxygen atoms form a fcc unit and $8 \mathrm{M}^{2+}$ bivalent ion and $16 \mathrm{Fe}^{3+}$ occupy interstitial sties, 8 tetrahedral (a) sites and 16 octahedral sites (b).

In a normal spinel, $\mathrm{M}^{2+}$ ions are located at the (a) sites, and $\mathrm{Fe}^{3+}$ ions at the (b) sites; Sometimes, all $\mathrm{M}^{2+}$ ions are located at (b) sites while the $\mathrm{Fe}^{3+}$ ions are equally distributed between (a) and (b) sites, which is called an inverse spinel. In many cases, intermediate cation distribution has been observed. The degree of inversion depends on the preparation, particularly on the cooling rate after sintering. Meanwhile, spinel ferrites are usually non-stoichiometric, which plays an important role in the magnetic properties, e.g. anisotropy, magnetostriction, and even Curie temperature $[20,30]$.

In summary, the interpreting of the magnetoelastic coupling in spinel ferrites is not a simple task, due to the interplay of various parameters: electron states, crystallographic ordering, stoichiometry, inverse rate, oxygen state of ions.

The magnetostriction of Co ferrite has been studied in the Soviet Union [31, 32] and occasionally in the US [33,34]. It exhibits high magnetostriction $\left(\lambda_{100}=-920 \mu, \lambda_{111}\right.$ $=180 \mu[2])$ with an adequate Curie temperature (793K) [35] for the present application. However, the brittleness due to porosity, structural imperfections and its relative high magneto-crystalline anisotropy, restrict its wider applications.

The preparation of ferrite materials includes four basic stages: powder preparation, greenbody forming, sintering and finishing. First, the $\mathrm{Fe}_{2} \mathrm{O}_{3}$ and the other oxides are 
mixed and milled in ballmills. After drying, the powder is compacted by dry-pressing to obtain a greenbody. Finally, sintering is carried out at temperatures between 1000-1400C for 2-20 hours and in atmospheres of $\mathrm{O}_{2}, \mathrm{~N}_{2}$ or noble gas. A finishing process is usually needed to obtain a final piece with the required dimension. It was found that the preparation and heat treatment have strong effects on the magnetic properties of ferrites [35]. Some new methods have been used to improve the properties of ferrites, e.g. coprecipitation [36], sol-gel [37] method to prepare ultra-fine powder, hot isotatic pressing to increase the sintering density.

\subsection{Composite materials}

Many of our modern technologies require materials with unusual combinations of properties that cannot be met by the conventional metal alloys, ceramics, or polymers. For example, low density and high strength, high hardness and impact strength. The development of composite materials has extended the materials property combination and ranges.

In the present work, high magnetostriction, good mechanical properties, and corrosion resistance need to be combined together. As stated in section 3.2 and 3.3, both Terfenol and ferrite materials have high magnetostrictions with poor mechanical properties (brittle, corrosive). Therefore, composite materials are obvious choices. Composites are considered to be multiphase materials that exhibit a significant proportion of the properties of both constituent phases such that a better combination of properties is realized. A compromise or "trade-off" in properties is usually made for many composites. 
Composites are composed of two or more phases. Generally, one is termed the matrix, which is continuous and surrounds other phase(s), often called the dispersed phase(s).

From the materials science point of view, there are three criteria to consider for composite materials. First, in order to obtain good composites, the match of thermal expansion coefficients of matrix and dispersed materials are always required, especially for those materials manufactured and/or used in different temperatures. The thermal strain will produce high internal stresses, even cracks after the composite undergoes environmental temperature change if the thermal expansion coefficients do not match. Second, the matrix material should be chemically compatible with the dispersed phase(s) under the conditions to fabricate and operate the composite material, i.e. no detrimental reactions occur between the matrix and dispersed phases(s). Third, similar elastic moduli among the components is advantageous.

From the magnetics point of view, magnetic interaction needs to be taken into account. For instance, magnetic shielding can occur between materials with different permeability. The presence of a second phase usually increases the domain wall pinning density, thus usually decreases the magnetic sensitivity. The magnetic properties would be more complex if there is magnetic coupling among components. 


\section{MAGNETOMECHANICAL EFFECT AND ITS APPLICATIONS}

\subsection{Villari effect - Magnetomechanical effect under uniaxial stress}

While the magnetostriction is known as Joule effect, the inverse effect, that is the stress dependence of the magnetization, is usually named after Villari's work [2] and called the Villari effect.

A summary of this effect was given by Bozorth [16]. Recently, there has been a resurgence of interest in the magnetomechanical effect [39-46]. It is now well recognized that stress is one of the major factors which influence the magnetic properties of magnetic materials and is comparable to magnetic field and temperature in its influence. Although the saturation magnetization in all materials is unaffected by a stress within the elastic limit, the stress has a strong effect on magnetization processes. For those materials which have positive magnetostriction, the permeability is increased by tension and decreased by compression. For instance, Permalloy containing $68 \%$ nickel has positive magnetostriction and high strain sensitivity. The maximum permeability is increased sevenfold by a stress of 20MPa. In those materials which have negative magnetostriction, the magnetization is increased by compression and there is a corresponding decrease in permeability with tension. Nickel is a typical example of a material having negative magnetostriction. Hysteresis loops of nickel and 68 Permalloy are shown in Fig. 4-1. The effects of stress on polycrystalline iron samples are complicated because the magnetostriction changes sign from low magnetic field to high magnetic field. 


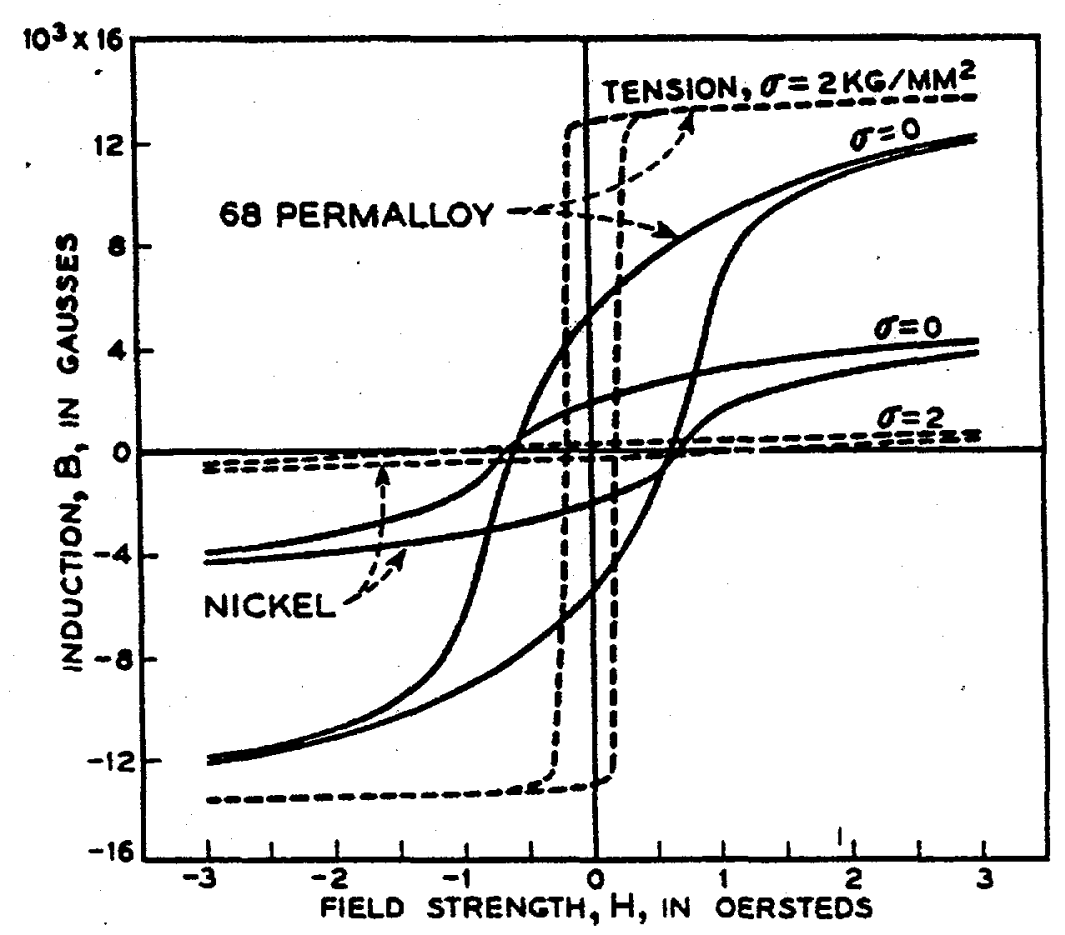

Fig. 4-1 Effect of stress on the hysteresis loops nickel and 68 Permalloy (after Bozorth [16]).

The magnetoelastic energy associated with the stress in magnetic materials is derived by Bozorth [16].

$$
\mathrm{E}_{\sigma}=\frac{3}{2} \lambda_{\mathrm{s}} \sigma \sin ^{2} \theta
$$

where $\lambda_{s}$ is the saturation magnetostriction and $\theta$ is the angle between magnetization and the stress $\sigma$.

Jiles [38] considered the effect of Poisson's ratio of materials, and then the energy relating to the magnetostriction was written by 


$$
E_{\sigma}=-\frac{3}{2} \lambda \sigma\left(\cos ^{2} \theta-v \sin ^{2} \theta\right)
$$

where $v$ is the Poisson's ratio of the material. Note in (4-2), $\lambda$ is the magnetostriction as a function of magnetization rather than simply the saturation magnetostriction. Therefore, this equation can be used in more cases.

Both the Bozorth and Jiles magnetostriction energy expressions show that the energy is zero when the stress is aligned parallel with the magnetization. If the magnetostriction is positive and the stress is in tension, the energy is a minimum in this position. The domains will tend to rotate to the direction of tension and consequently the component of magnetization along the tension direction is increased by the tension and tends to orient perpendicular to the axis of compression. Similarly, there exists a converse effect when the magnetostriction is negative.

Bozorth [16] and Cullity [19] also discussed the thermodynamic relation between magnetostriction and the magnetomechanical effect. For small reversible changes, there exists a relation,

$$
\left(\frac{\partial \lambda}{\partial H}\right)_{\sigma}=\left(\frac{\partial B}{\partial \sigma}\right)_{H}
$$

In practice, there are two distinctly different processes which can occur during the magnetomechanical effect. The first is called the $\sigma-\mathrm{H}$ process [16], which concerns the change of hysteresis curves that result from a constant stress, as shown in Fig. 4-1. Another process is $H-\sigma[38,39,46]$, in which the magnetization of a magnetic material changes with a change of stress under constant applied magnetic field, even without 
applied field, i.e. at remanence state. The $H-\sigma$ process has appeared to be more complex than $\sigma-\mathrm{H}$ process, however, is more suitable for magnetic sensor applications as described in chapter 6.

\subsection{Inverse Wiedemann effect - magnetomechanical effect under torsional stress}

4.2.1 Direct and inverse Wiedemann effect [2]

The direct Wiedemann effect occurs when a ferromagnetic rod is simultaneously magnetized by a longitudinal field and a circular field generated by a longitudinal current, the resulting helical magnetic field causes the sample to be twisted [2]. There is more than one definition of the inverse Wiedemann effect.

1. When a ferromagnet with longitudinal magnetization is subjected to a mechanical torque, the longitudinal magnetization changes and a circular magnetization is observed. Or, similarly, when a ferromagnet with circular magnetization is submitted to a torque, the circular magnetization changes and a longitudinal magnetization occurs.

2. When a ferromagnet is subjected to a torque, a longitudinal electric field associated with the change of the circular magnetization appears, i.e. a voltage appears between the ends of the sample (also called Matteucci effect).

The inverse Wiedemann effect is now receiving increased attention because of its relevance to magnetic torque sensors $[7-9,11-13,47-51]$. In the present work, the change of magnetization/magnetic properties of a magnetic material (either a rod or a ring) under a torque has been studied. Both axial and circular initial magnetizations were investigated. 


\subsubsection{Stress distribution under torque}

When a rod (or a ring) undergoes a torque $\tau$ in the plane right to its axis, the torsion can be considered as created by an inhomogeneous field of orthogonal stresses applied at $\pm 45^{\circ}$ from the axis, as shown in Fig 4-2. The stress can be calculated by

$$
\sigma=2 \frac{\tau r}{\pi R^{4}}
$$

where $\mathrm{R}$ is the radius of the rod and $\mathrm{r}$ is the distance from the axis. Thus, the magnetomechanical effect under torsional strain can be treated as the sum of the effects caused by two uniaxial stresses [49].

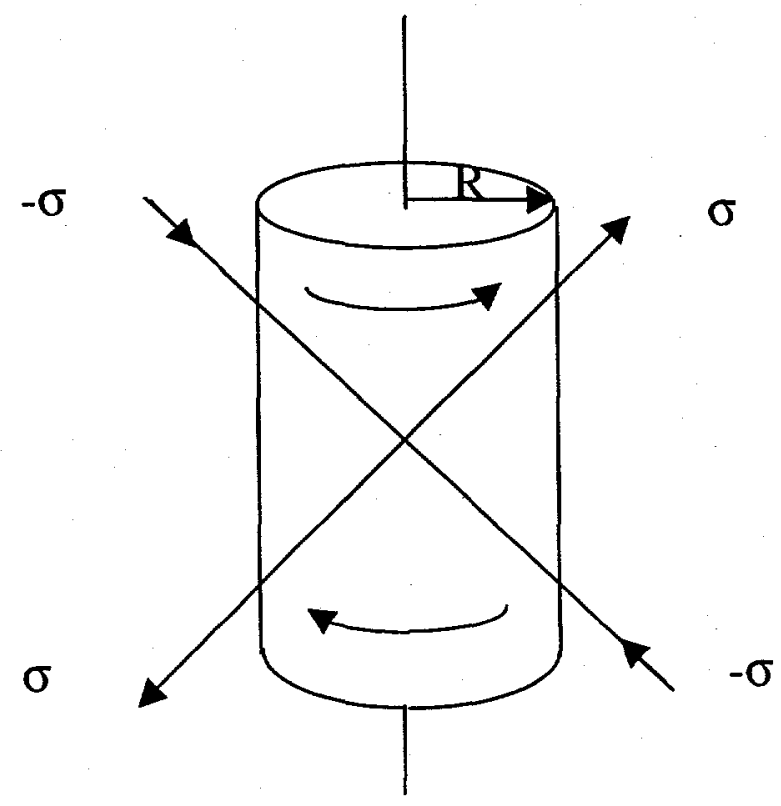

Fig.4-2 Distribution of the stresses in a rod under a torque. The torsional stress can be decomposed into biaxial stress, where each stress is perpendicular to the other and has 45 uniaxial stresses 


\subsubsection{Torque dependence of the magnetization}

In the demagnetized state, neither longitudinal nor circular magnetization can be observed under a torque. But when the sample is magnetized either by an axial field or a circular field, the torque induces both a longitudinal and circular magnetization [2]. The magnetization process may be either magnetic domain rotation or domain wall motion, or both. In the process, magnetization changes not only its direction but also its magnitude as well. As we noticed, both compressive and tensile stresses act on the material and the stress field is not uniform from the center to the surface. Hence, the torque dependence of the magnetization is much more complicated than that of uniaxial stress.

\subsubsection{Application for torque sensors}

The inverse Wiedemann effect tells us that torque can change magnetization of magnetostrictive materials. If the change of magnetization is linear and the signal is large enough, it can be used for torque sensing. The advantageous features of a magnetic torque sensor include, contactless measurement, rapid response, stability, accuracy, and high overload capacity [23]. Non-contact magnetic torque sensors have been considered to be perhaps the most suitable means of transferring information between computers and mechanical systems such as robotic systems and automobile applications [7, 12]. Such a torque sensor could make it possible for electromechanical steering systems to replace the costly and fuel inefficient hydraulic power steering systems currently in use. 
In order to improve the performance of magnetostrictive torque sensor, different materials and sensing modes have been tried [7-9, 11-13, 52]. Most conventional magnetic torque transducers sense the change of permeability or hysteresis parameters with excitation coils under different torques $[7-9,11-13,48]$. In earlier studies $[7,8]$, magnetic torque sensing was investigated directly on steel shafts using an external magnetic yoke carrying a driving coil and pickup coil. This kind of sensor has a narrow dynamic range (small stress and small magnetic induction) because steels exhibit small magnetostriction and which also changes sign at high magnetic induction. The accuracy is also limited by induced eddy currents in the shaft. Magnetostrictive amorphous alloys were suggested as the new material for developing torque sensors [11-13]. The structure is shown in Fig. 4-3. An amorphous ribbon or a group of ribbons were glued on the shaft under torque. A sensing coil generates the magnetic field and a pickup coil to sense the change of magnetic induction and thus the torque. This series of materials are usually $\mathrm{Fe}$ Si-B based Metglas ${ }^{\mathrm{TM}}$ alloys. They are made by cooling the molten alloy at a rate higher than $10^{6}{ }^{\circ} \mathrm{C} / \mathrm{s}$. As amorphous materials, they have very low anisotropy and coercivity and thus exhibit wide dynamic range and good linearity. However, the excitation coils, or permanent magnets which provide the applied magnetic field make the sensor structure complicated and expensive. The excitation magnetic field also induces noises because of the relationship between excitation field and the magnetization of sensor.

Garshelis $[10,25]$ proposed a simple magnetized ring sensor, which did not require external excitation. A typical structure is shown in Fig. 4-4. The magnetostrictive material, maraging steel, was made in a ring-shape. The ring was magnetized 
circumferentially before the measurement was made at remanence state. The surface magnetic field from such a sensor was measured using a Hall probe as a function of torque. This sensing mode required that the materials should have good magnetomechanical response plus immediate coercivity to maintain a high magnetic remanence. The magnetostriction $(<30 \mathrm{ppm})$ and coercivity $(<10 \mathrm{~A} / \mathrm{m})$ were somewhat low for the practical torque sensor applications.

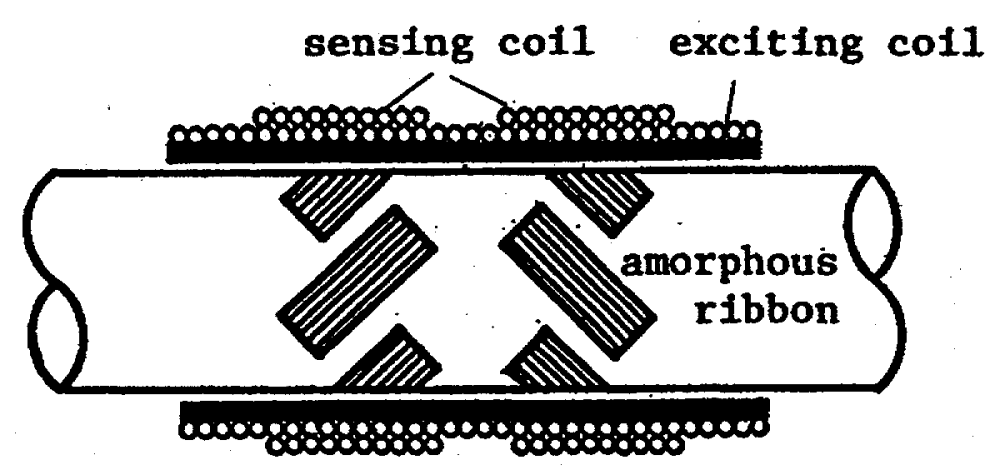

Fig. 4-3 Torque transducer using stress-sensitive amorphous ribbons (after Sasada [11]). Chevron-pattern of ribbons was used to improve the output.

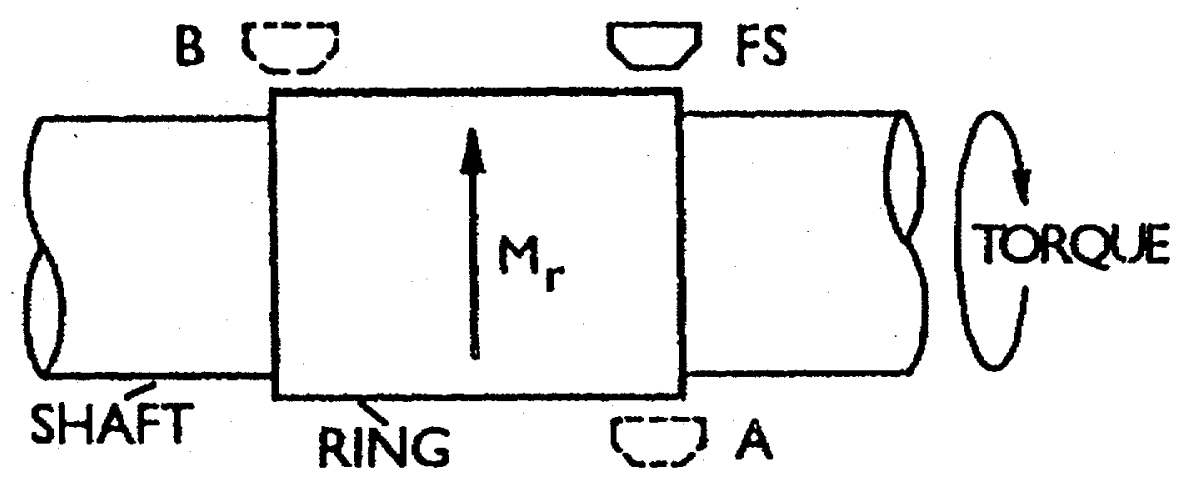

Fig. 4-4 Torque sensor using a maraging steel ring and a hall element (after Garshelis [53]) 


\section{EXPERIMENTAL PROCEDURE}

\subsection{Preparation of Terfenol-based composites}

The Terfenol-based composites were prepared by blending powders in an argon atmosphere. The composition of Terfenol-D particles used in this study was $\mathrm{Tb}_{0.3} \mathrm{Dy}_{0.7} \mathrm{Fe}_{2}$ while the matrix materials consisted of metal, resin and glass (soda-lime and phosphate). The metals were $\mathrm{Fe}, \mathrm{Cu}, \mathrm{Al}$ or $\mathrm{CeFe}_{2}$. Blended powder was poured into a $6 \mathrm{~mm}$ diameter die, pressed at 1 to $3 \mathrm{kN}$ under an inert argon atmosphere and heated at $10^{\circ} \mathrm{C} / \mathrm{min}$ to 300 $900^{\circ} \mathrm{C}$ depending on the matrix material. The load was removed and the material cooled to ambient temperature while still under argon atmosphere.

\subsection{Preparation of Co ferrite composites and magnetic properties measurement}

Co ferrite powder was prepared by blending stoichiometric amounts of fine, high purity cobalt oxide and iron oxide powders in a ball mill then firing at $1100^{\circ} \mathrm{C}$ for 72 hours in flowing dry air to form the compound. The Co ferrite compound powder was then milled to $<38 \mu \mathrm{m}$ and blended with chosen ratios of submicron metal powders (Ag, $\mathrm{Ni}, \mathrm{Co}$ ) to establish the metal bonded composite starting powder. These powders were cold pressed into shapes and sintered at up to $1450^{\circ} \mathrm{C}$. The resulting samples were typically cylinders of $5 \mathrm{~mm}$ diameter and $10-15 \mathrm{~mm}$ length. Coercivity and saturation magnetization were also measured for one of the samples. The specimen of composition 98 vol\% $\mathrm{CoO} \cdot \mathrm{Fe}_{2} \mathrm{O}_{3}+2$ vol\% $\mathrm{Ag}_{0.97} \mathrm{Ni}_{0.03}$, and dimensions $2 \times 2 \times 4.5 \mathrm{~mm}$ was measured along its long axis in a Quantum Design MPMS SQUID magnetometer. 


\subsection{Magnetostriction measurement}

All magnetostrictions in the present work were measured using strain gauges. The samples were cut in cylinders of $5 \mathrm{~mm}$ diameter and $10 \mathrm{~mm}$ length. Fine emery cloth $(600$ grit) was used to abrade the side surface of each cylindrical sample. One flat side face of $3.5 \mathrm{~mm}$ wide was obtained for bonding with a strain gage. Special adhesives were used to secure a good contact between the sample and the gages. Any air bubbles between the two faces to be glued would decrease the amplitude of the measured magnetostriction.

The magnetic field was applied parallel to the cylindrical axis of the specimens and the strain was measured along the same direction as the field was cycled in order to change the magnetization from positive saturation, through zero to negative saturation, then back through zero to positive saturation again. The magnetic field was measured using a standard Hall effect probe. Maximum magnetic field strength of $2 \mathrm{MA} / \mathrm{m}$ was applied to saturate the samples.

\subsection{Magnetic torque sensor test bed}

A magnetic torque sensor test bed as shown in Fig. 5-1 was developed as part of the present investigation and this was used to make the magnetomechanical measurements under torsional strain. The test bed provided pure applied torque with no associated bending moment, from $-10 \mathrm{~N} \bullet \mathrm{m}$ (counter clockwise, $\mathrm{CCW}$ ) to $+10 \mathrm{~N} \bullet \mathrm{m}$ (clockwise, $\mathrm{CW}$ ) using a computer-controlled servo motor (part 2 in Fig. 5-1). The precision of the applied 


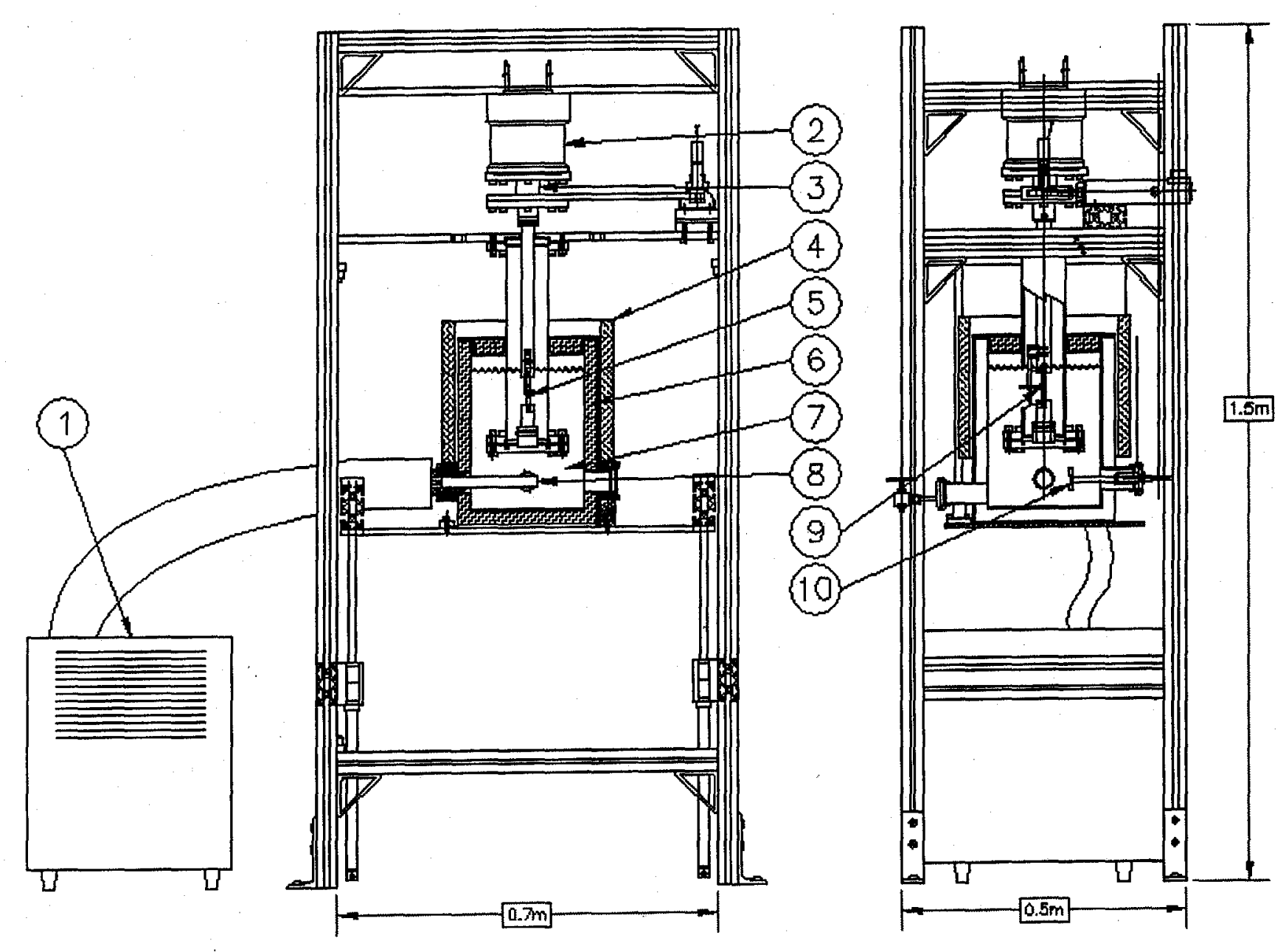

Fig. 5-1 The schematic diagram of magnetic torque sensor test bed

1.chiller, 2. servo motor, 3. commercial torque sensor, 4. Helmholz coil, 5. sample, 6 . liquid chamber, 7 fluid, 8 . cooling probe, 9 . Hall probe, 10. stirring propeller 
torque measured and monitored by a commercial torque sensor (part 3) was $0.05 \mathrm{~N} \bullet \mathrm{m}$. An applied magnetic field up to $\pm 2500 \mathrm{~A} / \mathrm{m}$ could be generated by a Helmholtz coil (part 4).

The chamber (part 6) was designed to contain heat-transfer fluids (part 7) to change the temperature of the sample. A stirring propeller (part 10) was used to increase the fluid circulation and improve the temperature homogeneity. The fluid used below ambient temperature was an ethanol water solution $(75 \mathrm{vol} \%$ ethanol $+25 \mathrm{vol} \%$ water $)$. The liquid was cooled by the cooling probe (part 8 ) of a commercial chiller (part 1), FTS FC80A11. Measurements between ambient temperature and $90^{\circ} \mathrm{C}$ were performed with the sample immersed in water which was heated in a separate tank, and circulated using a centrifugal pump. The magnetic-mechanical effect under torque could therefore be measured in a temperature range of $-40^{\circ} \mathrm{C}$ to $90^{\circ} \mathrm{C}$. During each measurement, the temperature maintained a fluctuation less than $\pm 0.2^{\circ} \mathrm{C}$.

The external magnetic field near the sample surface was measured using a Hall effect sensor and a Walker Gaussmeter with an IEEE 488 interface. The precision of magnetic field was $0.8 \mathrm{~A} / \mathrm{m}$. The distance of the Hall element from the sample surface was about $0.6 \mathrm{~mm}$. The magnetic induction of the sample was measured with a coil of 250 turns by a Walker flux meter. The control and data acquisition program was written using "Labview" software.

The interface of the program is shown in Fig. 5-2. This program can measure both $\mathbf{H}-\sigma / \tau$ process and $\tau / \sigma-\mathbf{H}$ process. In $\tau / \sigma-\mathbf{H}$ process, a torque was applied to the sample and then the hysteresis loop of the sample was measured. Then magnetic parameters (B, 


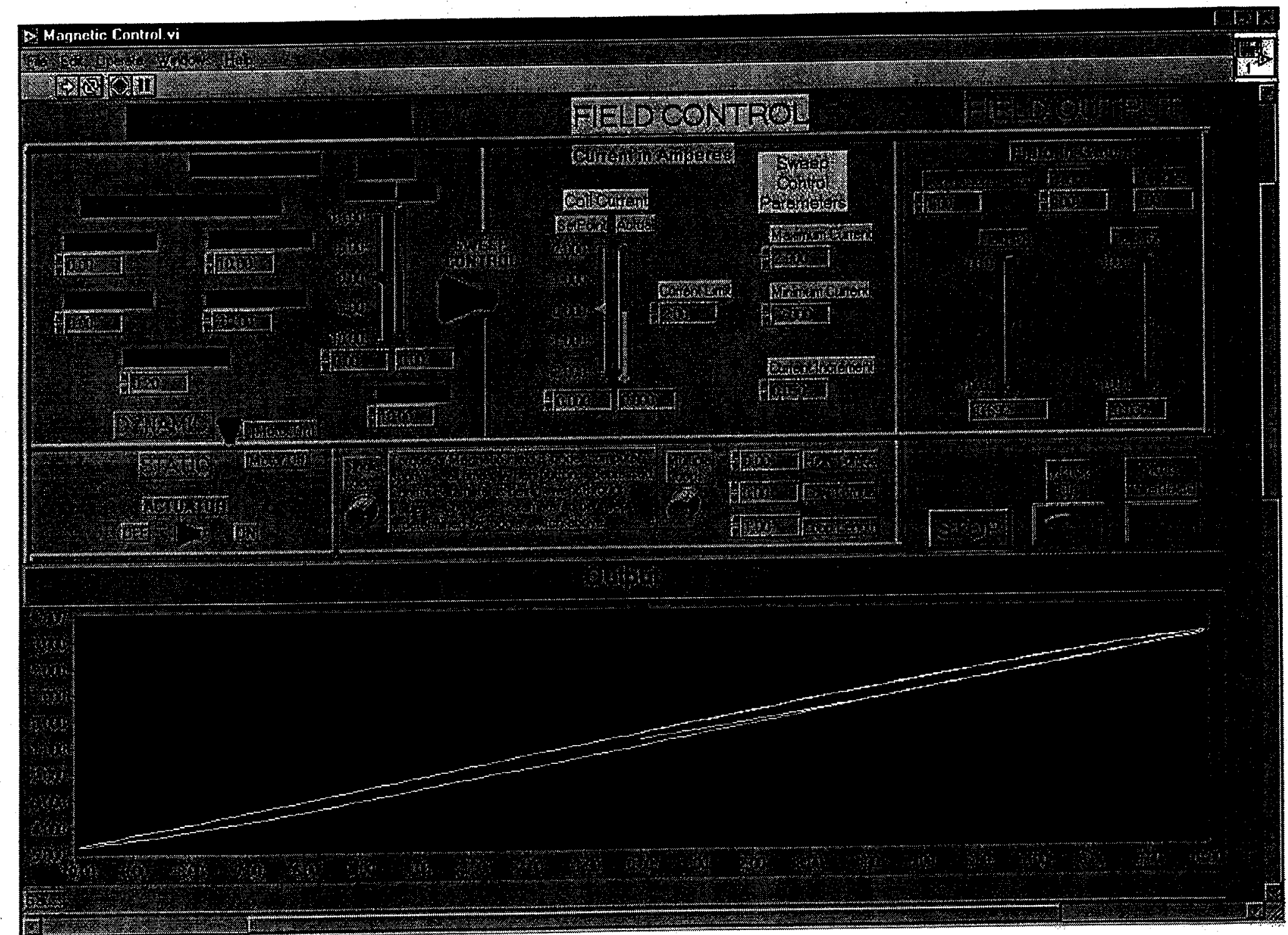

Fig. 5-2 The interface of the measurement program of the magnetic torque sensor test bed 
$H_{c}, B_{r}$ ) from those hysteresis loops at different torques were considered for torque sensing. In $\mathbf{H}-\sigma / \tau$ type processes, the applied magnetic field was kept constant. The applied torque was changed and the magnetic response measured. This included either change of surface magnetic field or magnetic induction/magnetization in the specimen. The program measured major loops (either magnetization Vs torque or $\mathbf{B}$ Vs $\mathbf{H}$ ), as well as minor loops. In a major loop, the applied field $\mathbf{H}$ or torque (horizontal axis) only reversed its change direction at the maximum value, i.e. the applied field/torque was increased from zero to the positive maximum, then reversed to negative maximum and back to zero. One major loop is referred to as a cycle. For minor loops, the value of horizontal axis reversed its direction at immediate values.

\section{$5.5 \mathrm{Fe}, \mathrm{Ni}$, Co rods and magnetomechanical effects measurement}

The dimensions of the $\mathrm{Fe}, \mathrm{Co}$ and $\mathrm{Ni}$ rods are shown in Fig.5-3. All these samples are electrolytic pure and were cast into cylindrical rods and machined to the desired shape. As-annealed samples were obtained with 24 hours annealing after machining. The samples are rods with length of $70 \mathrm{~mm}$ (3inches) and outer diameter of $13 \mathrm{~mm}(0.5 \mathrm{inch})$. Two square ends were used to fasten the sample to the shaft of the test bed and transfer the torque to the sample. Both $\mathbf{H}-\sigma$ and $\sigma-\mathbf{H}$ processes were measured. For $\sigma-\mathbf{H}$ processes, hysteresis loops (B vs $\mathbf{H}$ ) were measured at different torque values. The magnetic induction was measured using a coil wound around the sample. 
For $H-\sigma$ processes, the magnetic response was measured as the surface magnetic field. The samples were measured under an applied field of $2.5 \mathrm{kA} / \mathrm{m}$ or at remanence states. The remanence states were obtained after axial saturation of magnetization in an applied field of $53 \mathrm{kA} / \mathrm{m}$ before the magnetomechanical response was measured. The changes of external magnetic fields, including axial field $\left(\mathbf{H}_{\mathrm{a}}\right)$, and circumferential field $\left(\mathbf{H}_{\mathrm{cm}}\right)$, at the center of the rods were measured as a function of applied torque. For each sample, 10-100 torque cycles were tested. In each cycle, the applied torque was changed from 0 to $5 \mathrm{~N} \bullet \mathrm{m}(\mathrm{CW})$, then reversed to $-5 \mathrm{~N} \bullet \mathrm{m}(\mathrm{CCW})$ and then back to zero. For reference purposes, the magnetomechanical response was also measured in the demagnetized state.

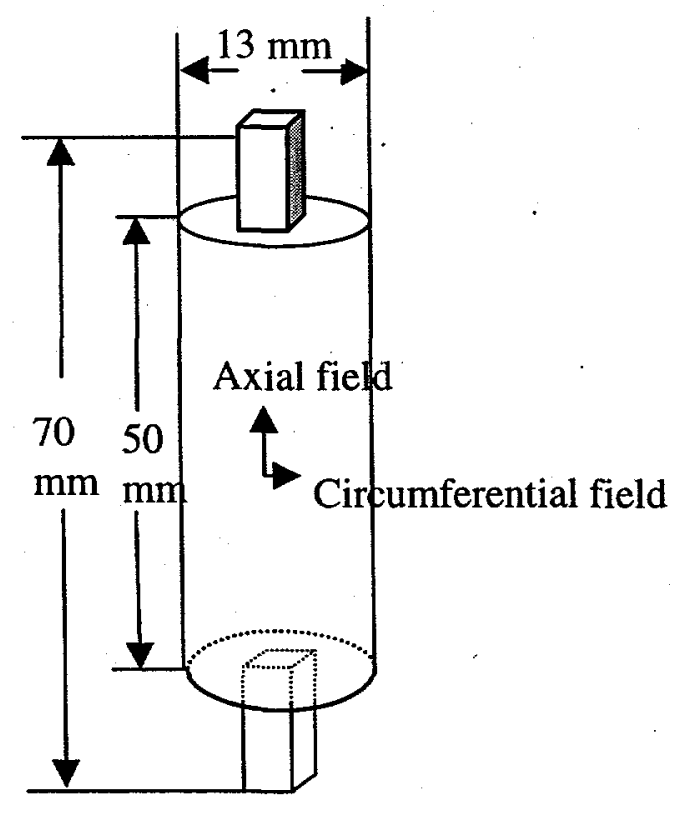

Fig. 5-3 Geometry of the samples. The torque was applied to the square ends. The surface magnetic fields were measured using a Hall effect sensor. The induction of the sample was measured using a flux meter. 


\subsection{Co-ferrite rings and measurement of magnetomechanical effects}

Selected compositions were pressed and sintered in the form of rings that were brazed on to stainless steel shafts. The configuration of the samples is shown in Fig. 5-4. The magnetic response to torque was measured using the magnetic torque sensor test bed. All samples were magnetized circumferentially to remanence before the torque response was tested. To achieve this, the ferrite rings were rotated in the fringing field of a narrowgap $(1 \mathrm{~mm})$ electromagnet, as shown in Fig.5-5, while the field was decreased from 160 $\mathrm{kA} / \mathrm{m}$ to 0 .

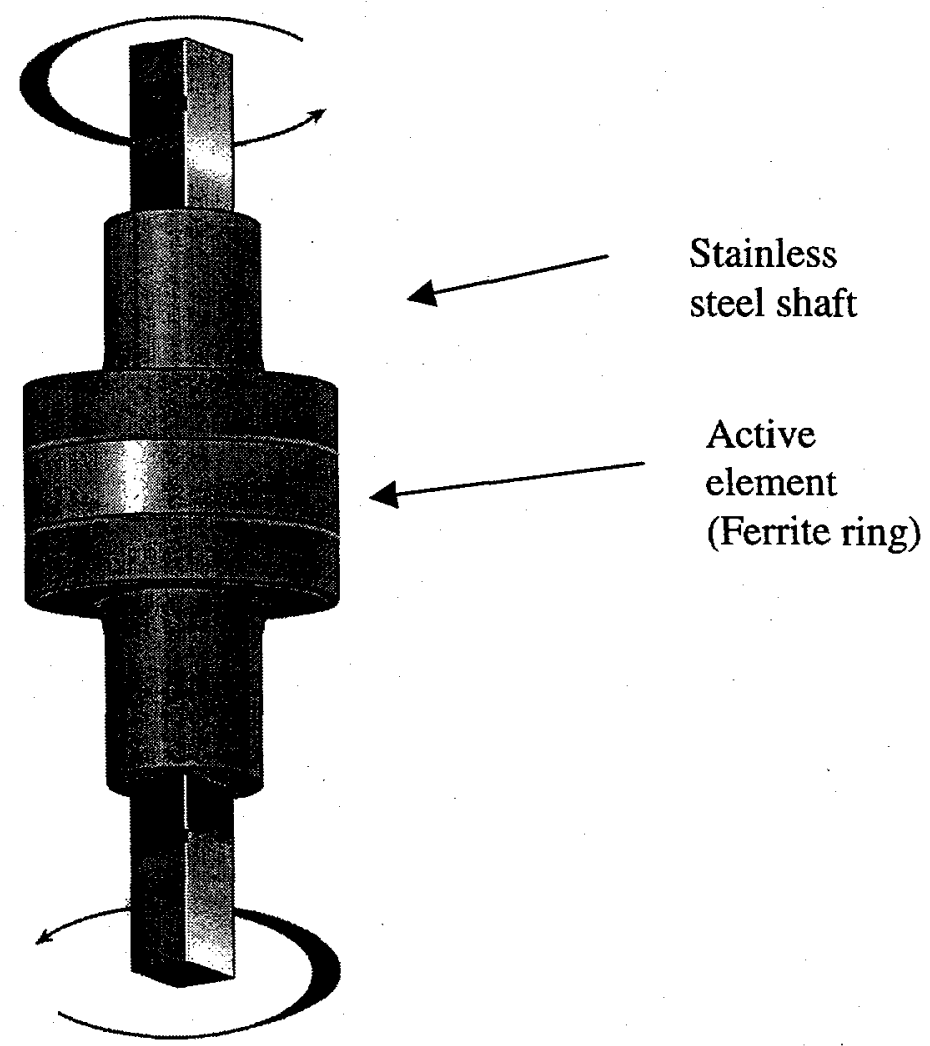

Fig. 5-4 The configuration of the ferrite ring samples bonded on stainless steel shaft 


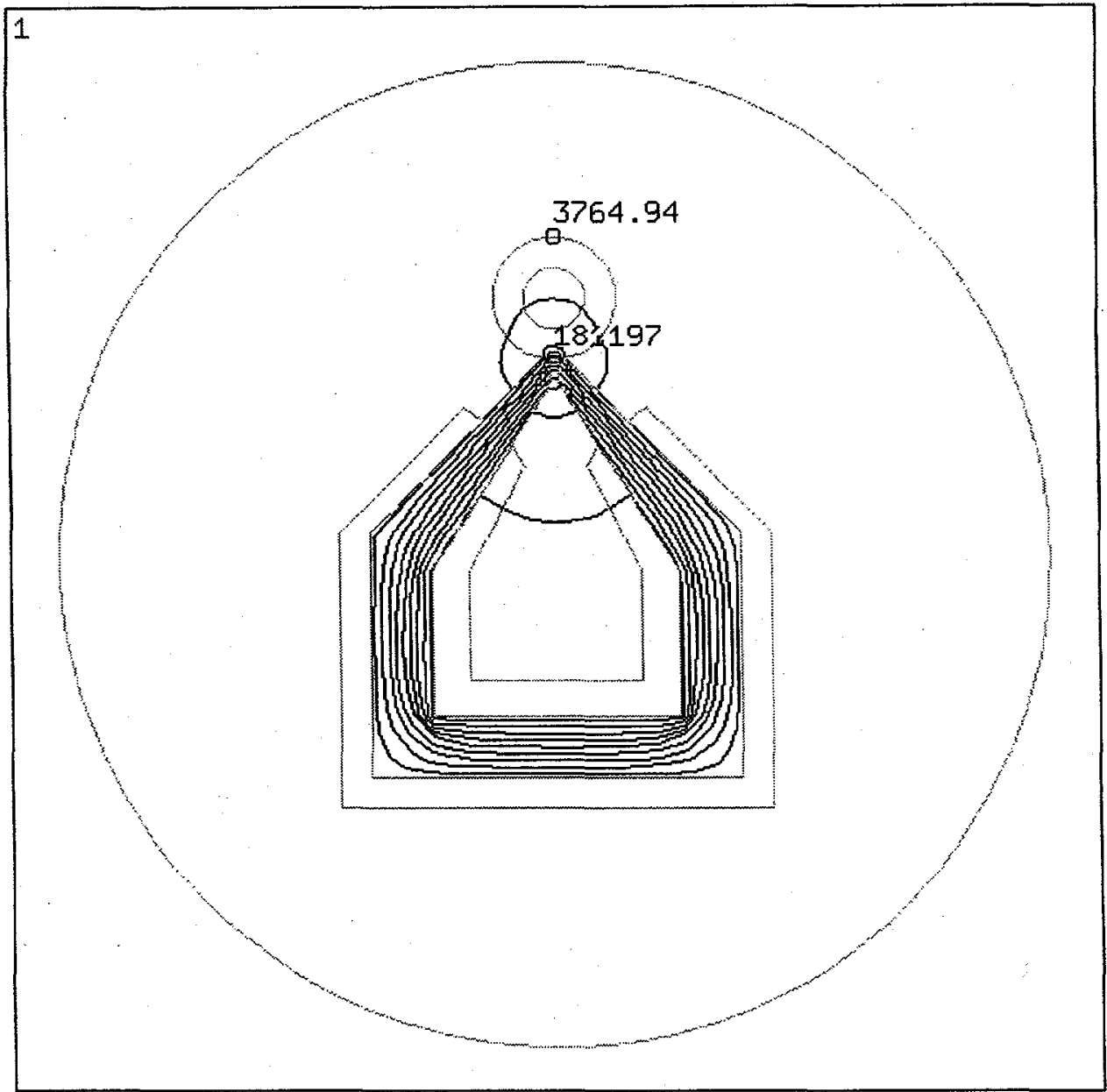

ANSYS 5.3

NOV 241998

$21: 59: 40$

NODAL SOLUTION

STEP $=2$

SUB $=1$

TIME $=2$

A2

RSYS $=0$

SMN $=-.003295$

$\operatorname{SMX}=.267376$

$A=.011742$

$\mathrm{B} \quad=.041817$

$\mathrm{C}=.071892$

$\mathrm{D}=.101966$

$E \quad=.132041$

$F=.162115$

$G=.19219$

$\mathrm{H}=.222264$

$I=.252339$

Fig. 5-5 The small-gap and high gradient electromagnet for magnetizing the ringshape Co ferrite circumferentially ( $g a p=1 \mathrm{~mm}$ ). The fringing field at the highest point of the ferrite ring, $3764 \mathrm{~A} / \mathrm{m}$ is less than the coercivity of the ferrite composites $(6-10 \mathrm{kA} / \mathrm{m})$.

The special yoke was designed based on FEM calculations. The material is high permeability soft iron. When a ring shape material is rotated on the top of the yoke, the fringing field is opposite the direction of the remanent magnetization at the highest point. Therefore, the yoke should have a small gap to generate a high gradient magnetic field so 
that the maximum field at the lowest point of the ring is strong enough to saturate or nearly saturate the ferrite and the maximum field at the highest point is less than the coercivity of the ferrite. The results of FEM calculation showed that the fringing field at the highest point of the ferrite ring, $3.7 \mathrm{kA} / \mathrm{m}$ is less than the coercivity of Co ferrite composites (generally $6-10 \mathrm{kA} / \mathrm{m}$ ).

After being magnetized circumferentially, the changes of external axial magnetic

fields, close to the surface at the midpoint along the axis, were measured as a function of applied torque in different routines (major, minor loops).

\subsection{Measurement of temperature dependence of magnetomechanical effects}

A chiller (FTS FC 80A11) manufactured by FTS Systems Inc. was used to cool the fluid in the chamber of the test bed. The lowest temperature of this chiller was $-80^{\circ} \mathrm{C}$. In order to obtain good fluid circulation, a fluid with a low freezing point $\left(<-50^{\circ} \mathrm{C}\right)$ and low viscosity is needed. In present work, ethanol-water solution (25 vol\% water) was chosen. The sample could be cooled down to $-37^{\circ} \mathrm{C}$ in 4 hours. The changes of external axial magnetic fields were measured as a function of applied torque (torque response) in the range of $-37^{\circ} \mathrm{C}$ to $23^{\circ} \mathrm{C}$.

Measurements between ambient temperature and $90^{\circ} \mathrm{C}$ were performed with the sample immersed in water which was heated in a separate tank, and circulated using a centrifugal pump. The torque response was measured at each preset point before it was heated to the next higher temperature. During each measurement, the temperature was 
kept constant to within $\pm 0.2^{\circ} \mathrm{C}$, which was monitored using two thermocouples. Finally, the torque response was measured after the sample had cooled back down to $22^{\circ} \mathrm{C}$.

In order to compare the temperature dependences between magnetomechanical hysteresis and M-H hysteresis, M-H hysteresis loops were also measured in the temperature range from $-40^{\circ} \mathrm{C}$ to $100^{\circ} \mathrm{C}$, using the Quantum Design MPMS SQUID magnetometer. 


\section{RESULTS AND DISCUSSION}

\subsection{Magnetostriction of Terfenol-based composites}

The rare-earth-iron compounds have been long recognized as highly magnetostrictive materials (room temperature magnetostriction, 1000-2000ppm) [4]. However, the high cost and brittleness of these materials restrict their applications. Terfenol-based composites were considered a potential solution to lower the cost and strengthen the material while maintaining adequate magnetostriction.

\subsubsection{Terfenol + soda lime glass}

Considering the chemical compatibility, a soda-lime glass matrix was chosen to bond the Terfenol particle. The magnetostrictions of this series of composites with different weight fractions of Terfenol are shown in Fig. 6-1. Compared with polycrystalline Terfenol, the composites exhibited much lower magnetostriction and particularly low $d_{33}$ coefficient at low applied field. It was usually considered that the second phase induces more domain wall pinning sites and additional anisotropy, thus inhibit the magnetization processes and reduces the initial permeability $[26,55]$.

Those composites with soda-lime matrix exhibited moderate saturation magnetostrictions (70-120ppm). However, they appeared to be very porous and had inadequate mechanical properties. It also required a relatively large fraction (40wt\%-50\%) of Terfenol to get acceptable levels of magnetostriction (>100ppm). 


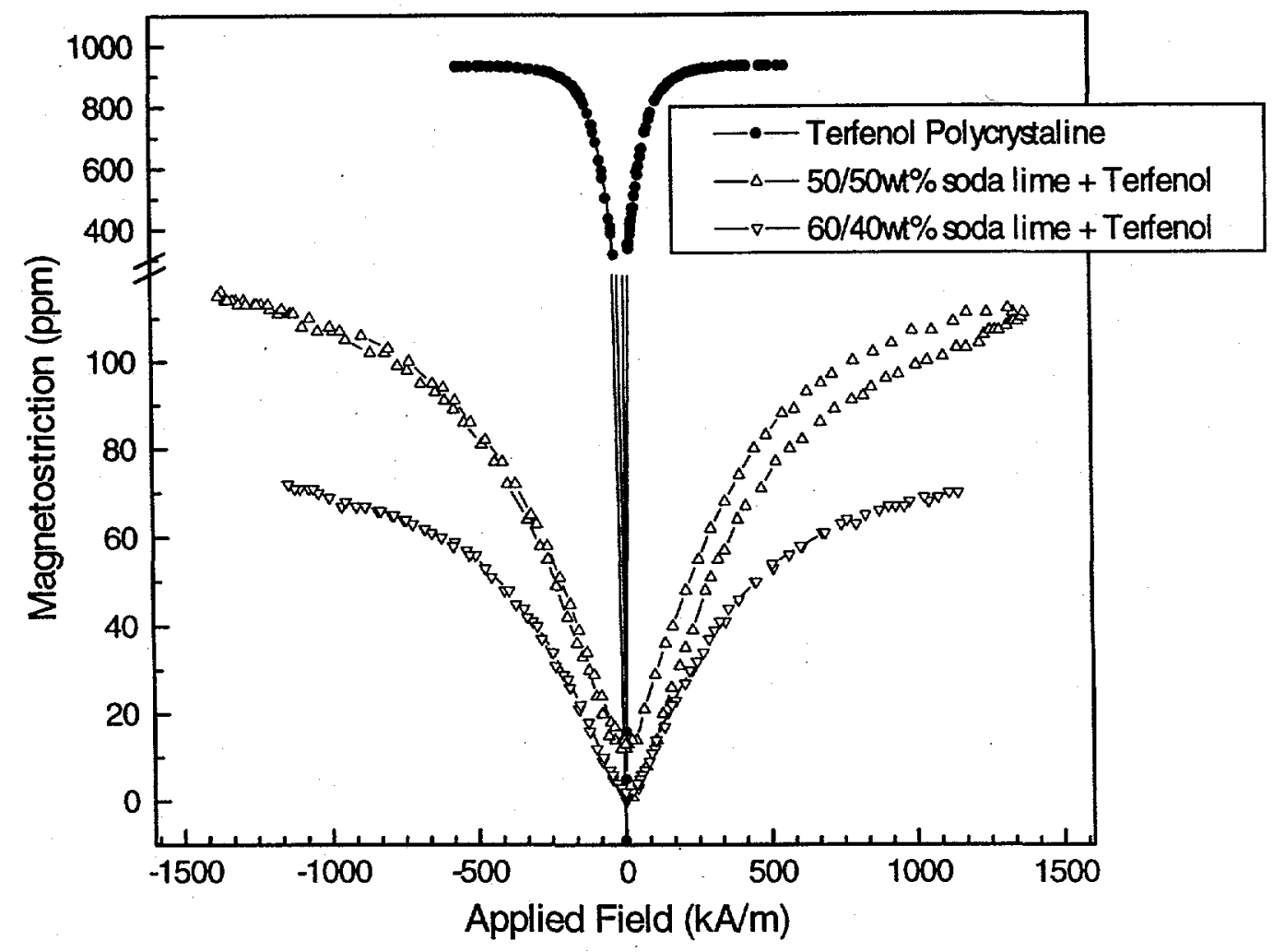

Fig. 6-1 Magnetostriction of Terfenol composites in soda lime matrix

Therefore, these materials are not considered ideal candidates for magnetostrictive sensor applications.

\subsubsection{Terfenol + resin}

Subsequently, polymer (resin) matrix materials were investigated. Embedding the Terfenol in a resin matrix can form a composite with low porosity which does not degrade the magnetic properties of the Terfenol phase through chemical reaction. 


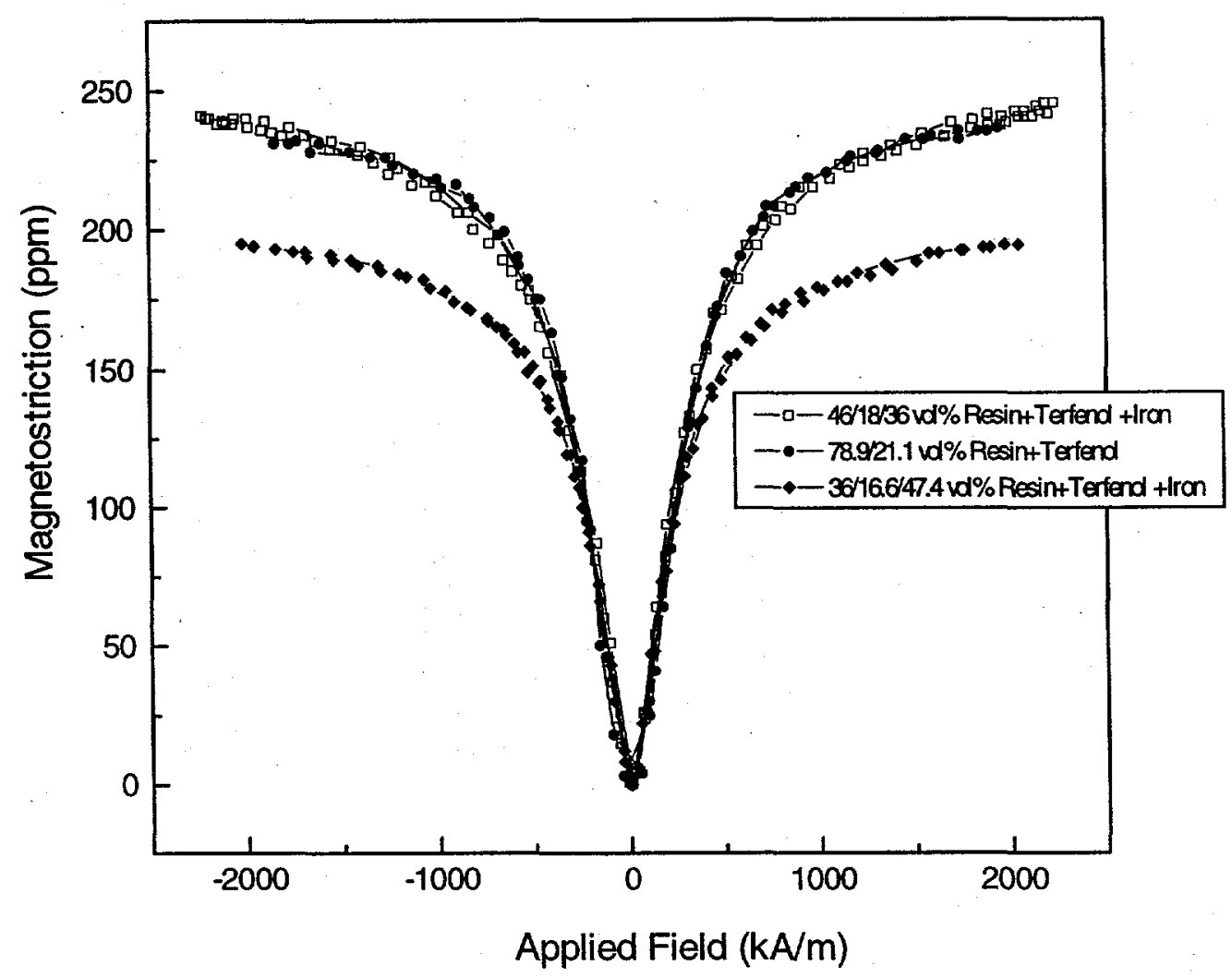

Fig. 6-2 Magnetostriction of Terfenol composites in resin matrix

The results are shown in Fig. 6-2. With 16-21 vol\% Terfenol in those composites, saturation magnetostrictions ranging from 200 to $250 \mathrm{ppm}$ were measured. Nevertheless, the mechanical properties (plastic deformation, creep, low tensile strength, low Young's modulus) of the resin are of questionable value for the application. Also, field strengths higher than $2 \mathrm{MA} / \mathrm{m}$ (almost the highest magnetic field generated using conventional methods in lab) were needed to achieve the saturation magnetostrictive strains, which implies that the magnetostriction was not sensitive to applied field. 


\subsubsection{Terfenol + iron $+\mathrm{NaPO}_{3}$ glass}

At processing temperatures lower than $600^{\circ} \mathrm{C}$, adequate saturation magnetostriction was obtained (100 150ppm), but final samples were porous. The results in Fig. 6-3 show that the magnetostriction increased with increasing volume fraction of Terfenol. In order to improve the density of the composites, the processing temperatures were increased to $900^{\circ} \mathrm{C}$. Despite this, the measured magnetostrictions were as low as $10-15 \mathrm{ppm}$.

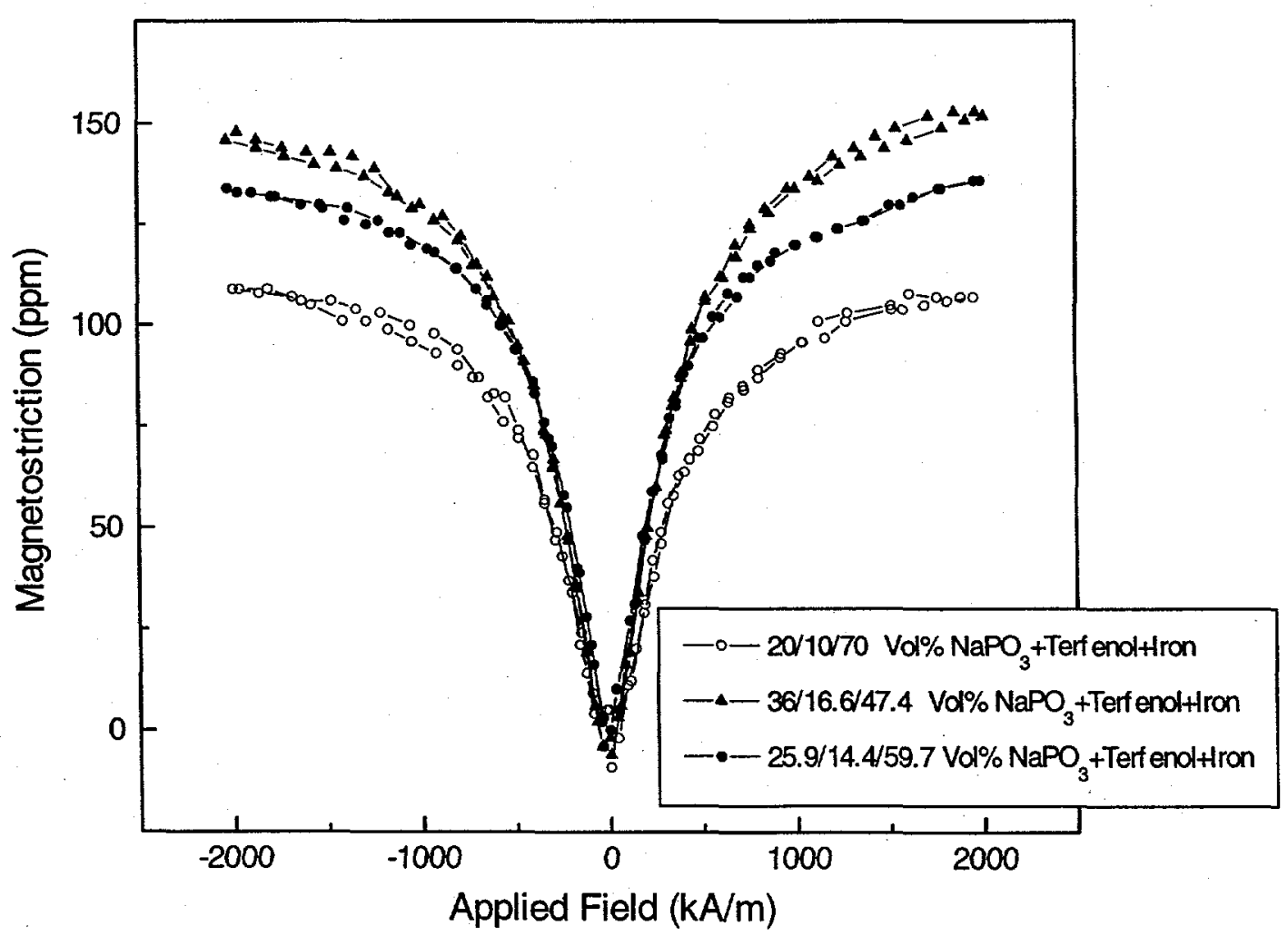

Fig. 6-3 Magnetostriction of Terfenol composites in $\mathrm{Fe}$ and $\mathrm{NaPO}_{3}$ matrix at low processing temperatures $\left(<600^{\circ} \mathrm{C}\right)$ 


\subsubsection{Terfenol + metal $(\mathrm{Fe}, \mathrm{Cu}, \mathrm{Al}, \mathrm{CeFe} 2)+\mathrm{NaPO}_{3}$ glass}

In order to investigate the effect of the modulus of the matrix, a series of samples with a fixed volume fraction $(10 \%)$ of Terfenol in matrix materials with different elastic moduli were measured. Magnetostriction curves of the series of composite materials are shown in Fig. 6-4. The matrix materials were: $\mathrm{A}\left(\mathrm{Fe}+\mathrm{NaPO}_{3}\right), \mathrm{B}\left(\mathrm{Cu}+\mathrm{NaPO}_{3}\right), \mathrm{C}(\mathrm{Al}+$ $\left.\mathrm{NaPO}_{3}\right), \mathrm{D}\left(\mathrm{CeFe}_{2}+\mathrm{NaPO}_{3}\right), \mathrm{E}\left(\mathrm{NaPO}_{3}\right)$. Magnetostriction of sample $\mathrm{A}$ was too low to be shown in the same figure. The Young's modulus of iron, copper, aluminum, $\mathrm{NaPO}_{3}$ glass, and $\mathrm{CeFe}_{2}$ are known to be $220 \mathrm{MPa}, 110 \mathrm{MPa}, 69 \mathrm{MPa}, 50 \mathrm{MPa}$, and $30 \mathrm{MPa}$, respectively [56]. These results show that the magnetostriction of the material increased with decreasing elastic modulus of the matrix.

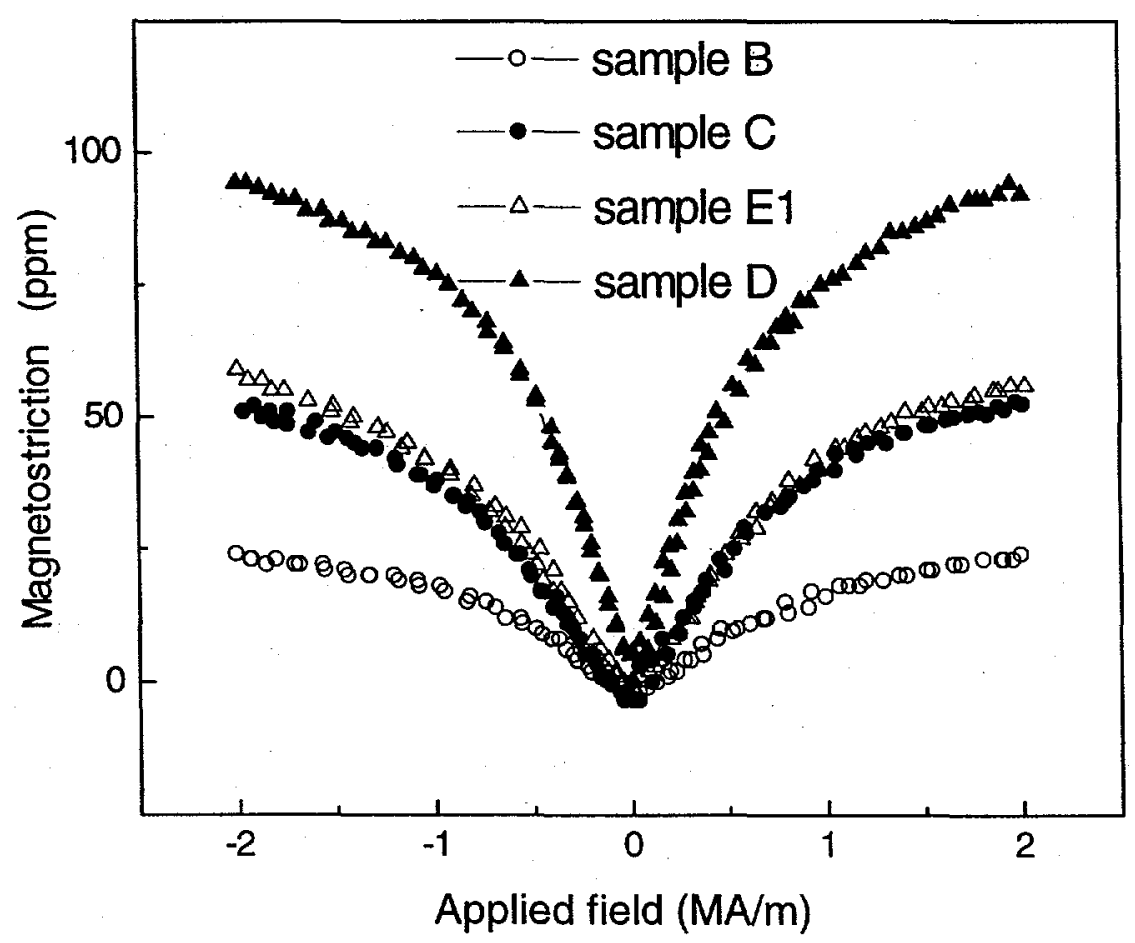

Fig. 6-4 Magnetostriction of several composites with equal volume fractions of Terfenol particles in different matrix materials 


\subsubsection{Terfenol $+\mathrm{NaPO}_{3}$ glass}

A series of samples with different volume fractions of Terfenol in the same matrix material, $\mathrm{NaPO}_{3}$ glass, were measured. Results shown in Fig. 6-5 indicate that the saturation magnetostriction of the material increased with increasing volume fraction of the magnetostrictive phase. This series of samples also appeared solid.

\subsubsection{Summary of magnetostriction of Terfenol-based composites}

Based on the above results, it appears that the elastic modulus of the matrix has a strong impact on the magnetostriction of magnetostrictive composites. The measured

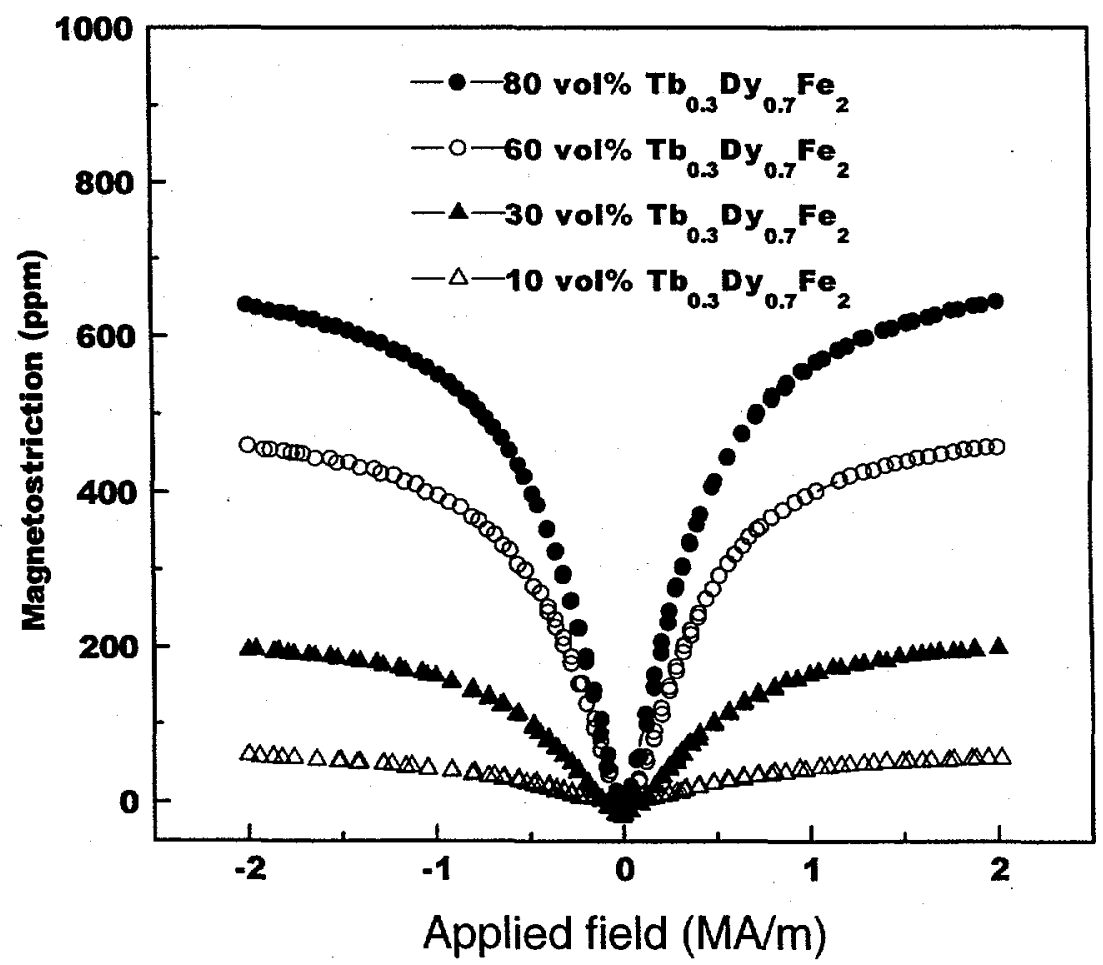

Fig. 6-5 Magnetostriction of Terfenol composites with different fraction of $\mathrm{NaPO}_{3}$ glass. 
saturation magnetostriction decreased from $90 \mathrm{ppm}$ to a value of $15 \mathrm{ppm}$ in composites with matrices with different elastic modulus. Higher elastic modulus was found to lead to lower magnetostriction.

Composites with $\mathrm{NaPO}_{3}$ glass matrix provided high magnetostriction/Terfenol fraction ratio together with good mechanical properties. However, initial piezomagnetic coefficients $\mathrm{d} \lambda / \mathrm{dH}$ of all the Terfenol-based composites are low. In addition, 30vol\% Terfenol is needed to obtain $200 \mathrm{ppm}$ in $\mathrm{NaPO}_{3}$ glass matrix composites, and as a rare earth intermetallic, the high content of rare earth metal makes the material extremely susceptible to corrosion.

\subsection{Magnetostriction of Co ferrite and its composites}

Polycrystalline Co ferrite $\left(\mathrm{CoO} \cdot \mathrm{Fe}_{2} \mathrm{O}_{3}\right)$ exhibits high magnetostriction $(\sim 200 \mathrm{ppm})$ at room temperature. But due to porosity [20], and relatively low mechanical properties (fracture strength) restricts its applications [57]. In the present study, $\mathrm{Ag} / \mathrm{Ni}$ or $\mathrm{Ag} / \mathrm{Co}$ was used as the metallic constituent in order to bind the ferrite together [58]. Ag, which has a low oxygen affinity, has the advantage of not reducing the ferrite. However, the addition of other elements such as $\mathrm{Ni}$ or $\mathrm{Co}$ is required, in order to cause the metal to wet the ferrite properly during fabrication. This wetting improves the mechanical properties of the Coferrite by producing a stronger bond. The presence of transition magnetic metals $(\mathrm{Co} / \mathrm{Ni})$ could also change the magnetic properties of the Co ferrite composite.

Maximum magnetostrictions of Co-ferrite and selected metal-bonded Co-ferrite composites are listed in Table 6-1. While some of the metal-bonded composites have 
lower peak magnetostrictions than pure $\mathrm{CoO} \cdot \mathrm{Fe}_{2} \mathrm{O}_{3}$, they generally offer better mechanical properties with fracture strength higher than $69 \mathrm{MPa}$ (10ksi). Furthermore, Co ferrite, being an oxide, has excellent corrosion resistance.

Besides the peak magnetostriction, the piezomagnetic coefficient (initial slope of magnetostriction curve, $\left.(\partial \lambda / \partial \mathrm{H})_{\sigma}\right)$ is another important parameter of magnetostrictive materials, especially for sensor materials [26]. The magnetostriction curves of a series of sample with $\mathrm{Ag} / \mathrm{Ni}$ additives are shown in Fig. 6-6. This series of samples had 2 vol\% $\mathrm{Ag} / \mathrm{Ni}$ metal additives to the $\mathrm{CoO} \cdot \mathrm{Fe}_{2} \mathrm{O}_{3}$. With careful adjustment of the composition of metal fraction of $\mathrm{Ag} / \mathrm{Ni}(97 \% \mathrm{Ag}$ and $3 \% \mathrm{Ni})$, higher initial piezomagnetic coefficients together with comparable peak magnetostrictions to Co ferrite were obtained.

Table 6-1

Magnetostrictions of $\mathrm{CoO} \cdot \mathrm{Fe}_{2} \mathrm{O}_{3}$ and various composites bonded with metals

\begin{tabular}{ll}
\hline \hline Composition & Maximum magnetostriction \\
\hline $100 \% \mathrm{CoO} \cdot \mathrm{Fe}_{2} \mathrm{O}_{3}$ & $-225 \mathrm{ppm}$ \\
$2 / 98 \mathrm{vol} \% \mathrm{Ag}_{0.1} \mathrm{Ni}_{0.9}+\mathrm{CoO} \cdot \mathrm{Fe}_{2} \mathrm{O}_{3}$ & $-168 \mathrm{ppm}$ \\
$2 / 98 \mathrm{vol} \% \mathrm{Ag}_{0.5} \mathrm{Ni}_{0.5}+\mathrm{CoO} \cdot \mathrm{Fe}_{2} \mathrm{O}_{3}$ & $-195 \mathrm{ppm}$ \\
$2 / 98 \mathrm{vol} \% \mathrm{Ag}_{0.8} \mathrm{Ni}_{0.2}+\mathrm{CoO} \cdot \mathrm{Fe}_{2} \mathrm{O}_{3}$ & $-210 \mathrm{ppm}$ \\
$2 / 98 \mathrm{vol} \% \mathrm{Ag}_{0.97} \mathrm{Ni}_{0.03}+\mathrm{CoO} \cdot \mathrm{Fe}_{2} \mathrm{O}_{3}$ & $-233 \mathrm{ppm}$ \\
$2 / 98 \mathrm{vol} \% \mathrm{Ag}_{0.98} \mathrm{Ni}_{0.02}+\mathrm{CoO} \cdot \mathrm{Fe}_{2} \mathrm{O}_{3}$ & $-205 \mathrm{ppm}$ \\
$1 \mathrm{vol} \% \mathrm{Co}+\mathrm{CoO} \cdot \mathrm{Fe}_{2} \mathrm{O}_{3}$ & $-155 \mathrm{ppm}$ \\
$2 \mathrm{vol} \% \mathrm{Co}+\mathrm{CoO} \cdot \mathrm{Fe}_{2} \mathrm{O}_{3}$ & $-80 \mathrm{ppm}$ \\
$5 \mathrm{vol} \% \mathrm{Co}+\mathrm{CoO} \cdot \mathrm{Fe}_{2} \mathrm{O}_{3}$ & $-25 \mathrm{ppm}$ \\
\hline \hline
\end{tabular}




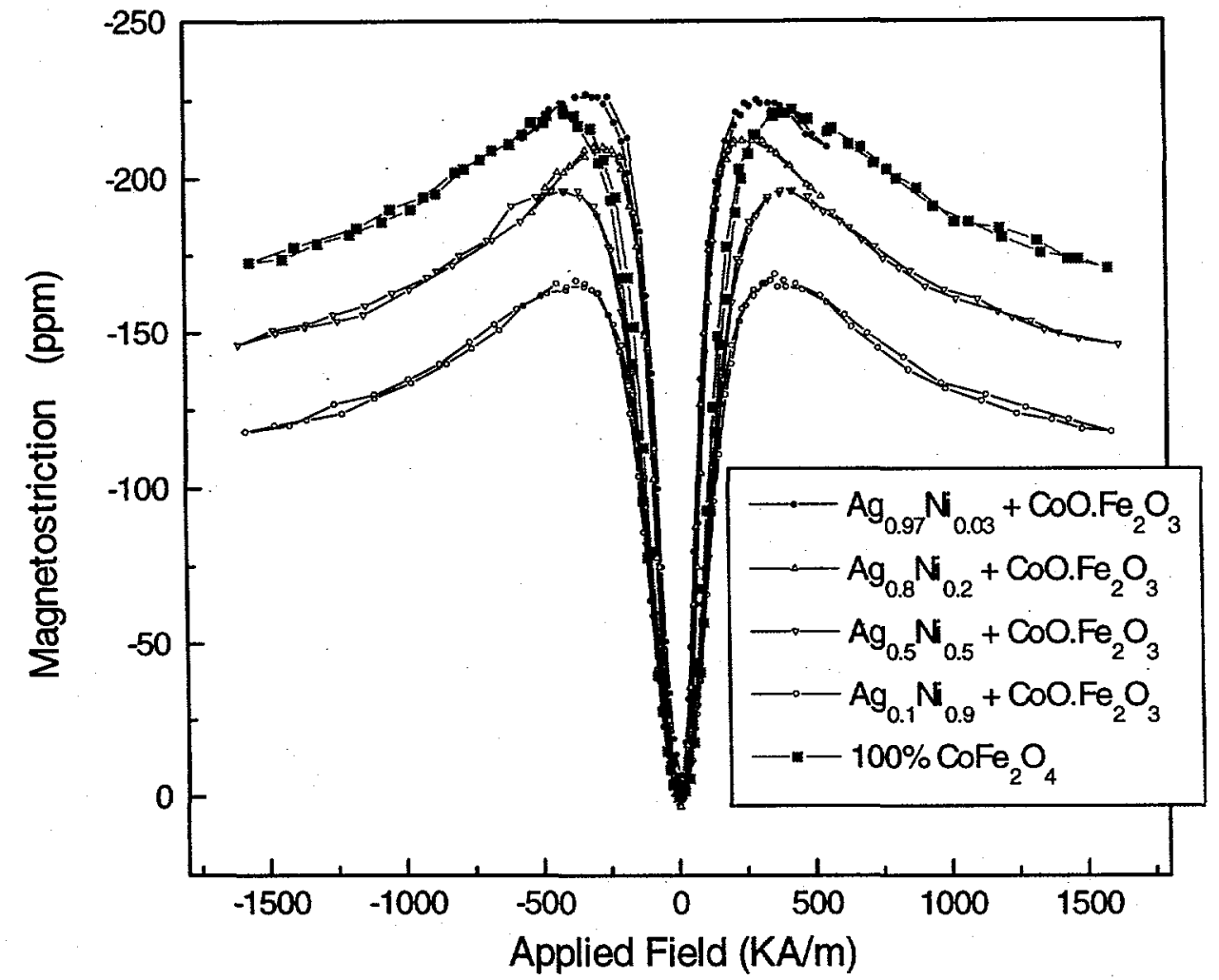

Fig.6-6 Magnetostrictions of Co-ferrite and selected $\mathrm{Ag} / \mathrm{Ni}$ - bonded Co-ferrite composites

Magnetostriction curves of another series of $\mathrm{Co}$ ferrite composites ( $\mathrm{Co}+$ $\mathrm{CoO} \cdot \mathrm{Fe}_{2} \mathrm{O}_{3}$ ) are shown in Fig. 6-7. While these composites have lower peak magnetostriction than $\mathrm{CoO} \cdot \mathrm{Fe}_{2} \mathrm{O}_{3}$, some of them exhibit higher initial piezomagnetic coefficients $\mathrm{d} \lambda / \mathrm{dH}$. The results show that the saturation magnetostriction decreased as the Co volume percentage increased from 0 to $5 \%$ while the Co additive increased the sensitivity to magnetic field, $\mathrm{d} \lambda / \mathrm{dH}$. 


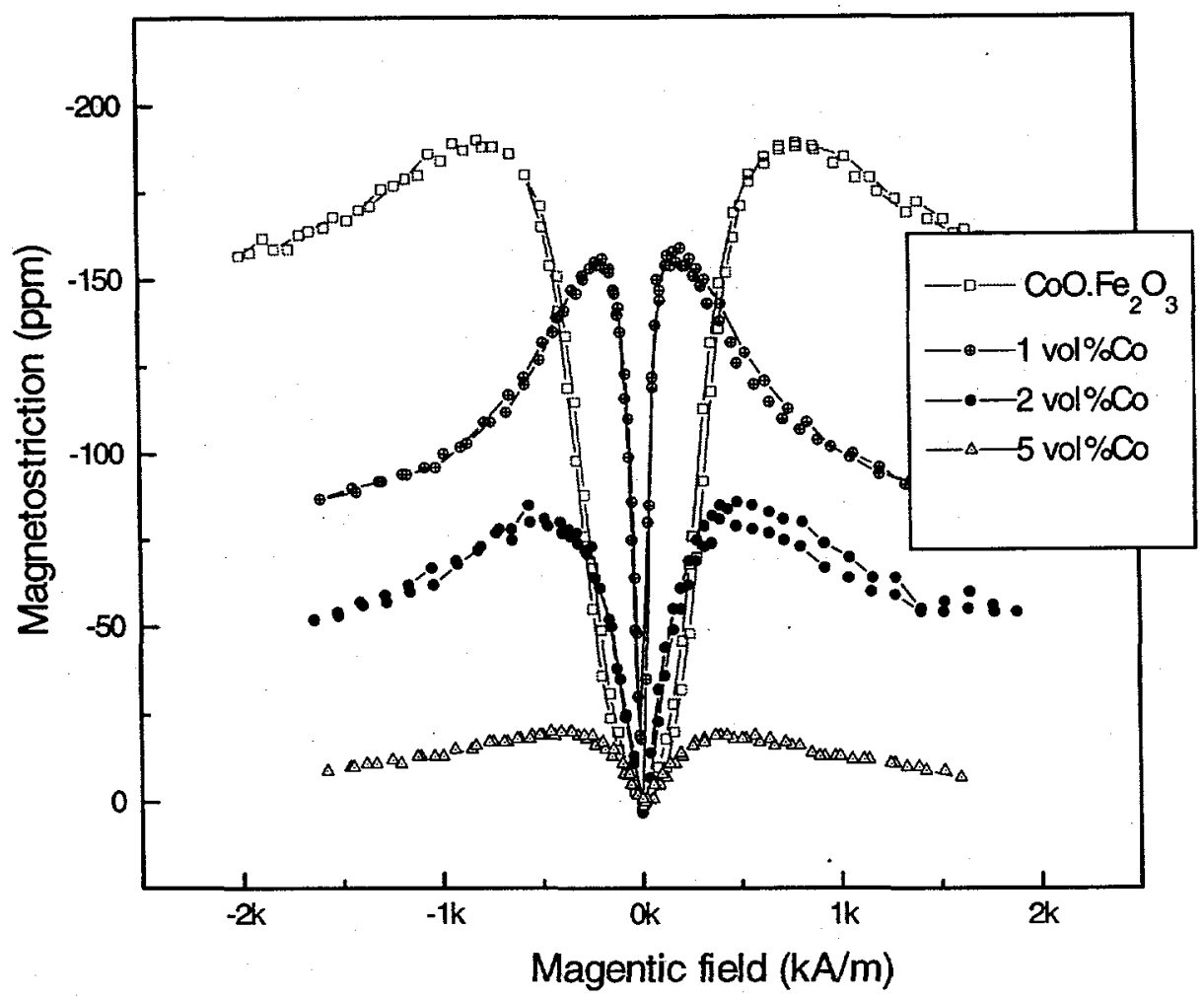

Fig 6-7 Magnetostriction of Co ferrite composite bonded with Co additive. Co decreased the anisotropy energy of the Co ferrite and then decreased the saturation magnetostriction and increased the piezomagnetic coefficient

In order to analyze the magnetostriction properties of metal-bonded Co ferrite composites, the hysteresis loops of those materials have been measured. The metal-bonded Co ferrite composites generally exhibited coercivities in a range of $6-8 \mathrm{kA} / \mathrm{m}$. This was much lower than that $(160 \mathrm{kA} / \mathrm{m})$ reported for Co ferrite $[34,59,60]$. Generally, there are two contributing factors to the coercivity of materials; anisotropy and domain wall pinning. As a second phase in ferrimagnetic materials, metal additives should increase the number density of domain wall pinning sites. Therefore, the decrease of coercivity must be induced by the decrease of anisotropy. 
From the magnetostriction point of view, the decrease of anisotropy energy leads to an increase of the initial permeability and thus initial piezomagnetic coefficient. However, the magneto-elastic coupling decreases at the same time because the anisotropy energy is strain-dependent. So, the peak magnetostriction of metal-bonded Co ferrites was usually lower than plain Co ferrite.

Usually, the presence of a nonmagnetic second phase in magnetic materials phase hinders the magnetization processes and decreases initial piezomagnetic coefficient, $\mathrm{d} \lambda / \mathrm{dH}$ in low fields. However, the metals in Co ferrite composites had the reverse effect. This can be explained since $\mathrm{Ni}$, Co changes the stoichiometry and $\mathrm{Co} / \mathrm{Fe}$ ratio in ferrite and thereby decreases the anisotropy of $\mathrm{CoO} \cdot \mathrm{Fe}_{2} \mathrm{O}_{3}$ because the anisotropy of spinel ferrite is stoichiometric and sensitive to the $\mathrm{Co} / \mathrm{Fe}$ ratio [30].

\subsection{Comparison of magnetostriction in Terfenol-based and Co ferrite composites}

The magnetostriction of a typical Co-ferrite composite with a composition of $2 \% \mathrm{Ag}_{0.97} \mathrm{Ni}_{0.03}+98 \mathrm{vol} \% \mathrm{CoO} \cdot \mathrm{Fe}_{2} \mathrm{O}_{3}$ was comparable in magnitude to that of Terfenolbased composite (30vol\% Terfenol $\left.+70 \mathrm{vol} \% \mathrm{NaPO}_{3}\right)$ in Fig.6-8. $\mathrm{CoO} \cdot \mathrm{Fe}_{2} \mathrm{O}_{3}$ is known to have a cubic spinel crystal structure with magnetic easy axes along the $<100>$ directions. The magnetostriction coefficients $\lambda_{100}$ and $\lambda_{111}$ have opposite signs, and the easy axis magnetostriction coefficient $\lambda_{100}$ is approximately 5 times the magnitude of $\lambda_{111}$ [20]. The measured magnetostriction of the $\mathrm{CoO} \cdot \mathrm{Fe}_{2} \mathrm{O}_{3}$ peaks at relatively low field $(200-300 \mathrm{kA} / \mathrm{m})$, then decreases with increasing field, as the magnetization of the particles is rotated away from easy directions. 


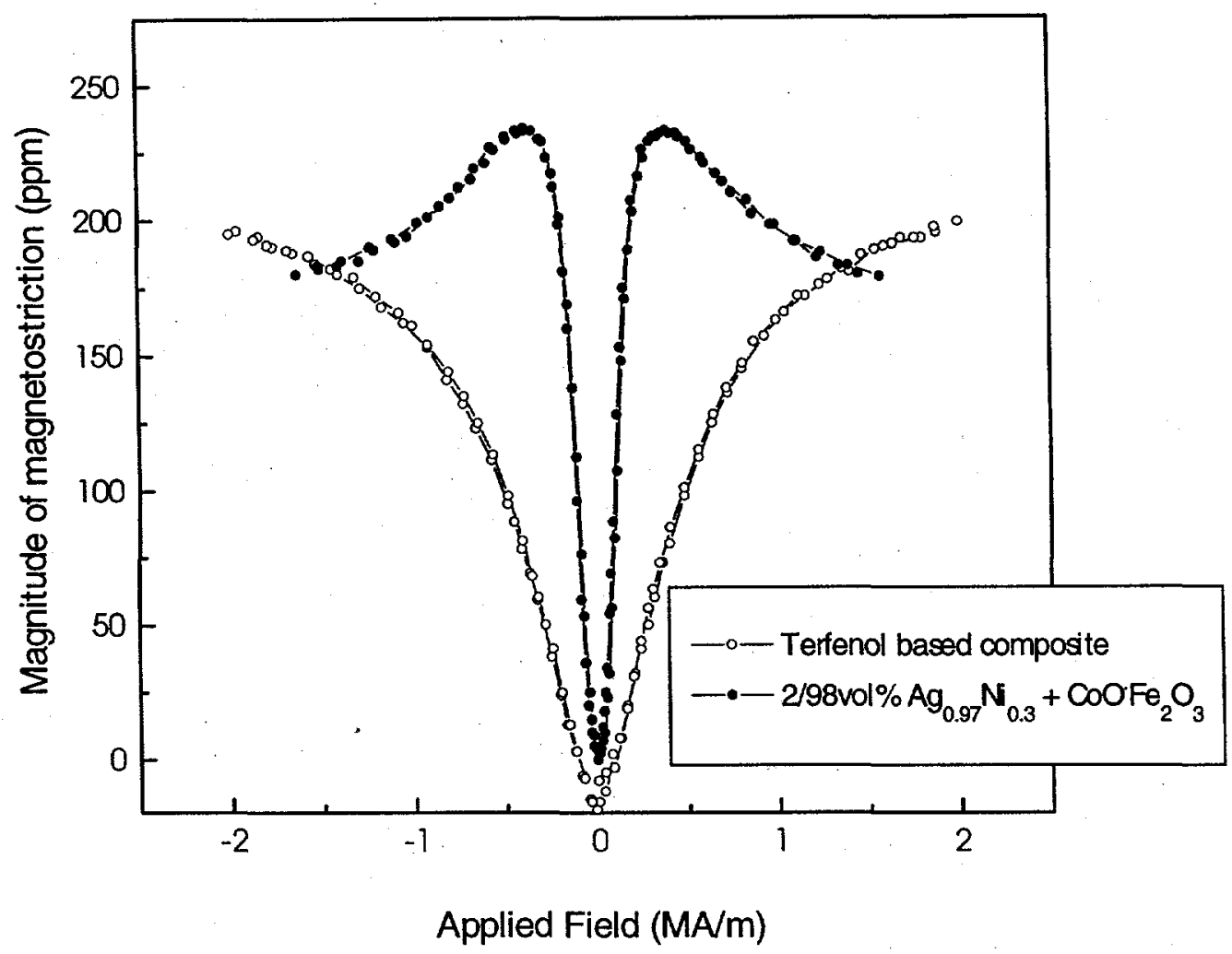

Fig. 6-8 Comparison of magnetostrictions of Co-ferrite composite and Terfenol composite

This maximum at low applied field contrasts with that of the Terfenol-based composites, which only saturate at very high applied fields $\left(2 \times 10^{6} \mathrm{~A} / \mathrm{m}\right.$ or larger). Coferrite and its metal-bonded composites also have strain derivatives $(\partial \lambda / \partial \mathrm{H})_{\sigma}$ of typically $1 \times 10^{-9} \mathrm{~A}^{-1} \mathrm{~m}$, at low applied fields $(<100 \mathrm{kA} / \mathrm{m})$, which are comparable to those of polycrystalline Terfenol [61], and much larger than those of Terfenol-based composites, which had values of typically $2 \times 10^{-10} \mathrm{~A}^{-1} \mathrm{~m}$.

The magnetostriction together with mechanical and corrosion properties of polycrystalline Terfenol, Co ferrite, Terfenol-based, and metal-based Co ferrite composites are compared in Table 6-2. Although the single crystalline and polycrystalline Terfenol can 
provide even higher magnetostriction (1000-2000ppm), its cost is much higher than Co ferrite composites. Furthermore, the mechanical and corrosive properties could not meet the requirements for sensor applications. The Terfenol-based composites studied in the present work lowered the cost and improved the mechancial properties. However, the low piezomagnetic coefficient implies low stress sensitivity for torque sensing. Metal bonded Co ferrite exhibits the optimum combination of sufficient magnetostriction, high piezomagnetic coefficient, very good mechanical properties, corrosion resistance, and low cost. The prime characteristics of this series of materials appear promising for magnetomechanical sensing and actuating applications.

Table 6-2 Comparison of Terfenol, Terfenol composite and Co-ferrite composite

\begin{tabular}{lccccc}
\hline \hline & $\begin{array}{c}\text { Initial slope } \\
\frac{d \lambda}{d H}\left(\mathrm{~A}^{-1} \mathrm{~m}\right)\end{array}$ & $\begin{array}{c}\text { Saturation } \\
\text { magnetostriction } \\
\left(\times 10^{-6}\right)\end{array}$ & $\begin{array}{c}\text { Cost/gram } \\
(\$)\end{array}$ & $\begin{array}{c}\text { Mechanical } \\
\text { properties }\end{array}$ & $\begin{array}{l}\text { Corrosion } \\
\text { resistance }\end{array}$ \\
\hline $\begin{array}{l}\text { Polycrystalline } \\
\text { Terfenol }\end{array}$ & $1.3 \times 10^{-9}$ & 1000 & 15.00 & Poor & poor \\
$\begin{array}{l}\text { Terfenol-based } \\
\text { Composite }\end{array}$ & $\begin{array}{c}2 \times 10^{-10} \\
(30 \mathrm{vol} \%)\end{array}$ & 200 & 5.00 & Good & good \\
$\begin{array}{l}\text { Co ferrite } \\
1.0 \times 10^{-9}\end{array}$ & 225 & 0.75 & Poor & excellent \\
$\begin{array}{l}\text { Metal-bonded Co } \\
\text { ferrite composite }\end{array}$ & $1.3 \times 10^{-9}$ & 230 & 0.75 & very good & excellent \\
\hline \hline
\end{tabular}

* Composition: $2 \% \mathrm{Ag}_{0.97} \mathrm{Ni}_{0.03}+98 \mathrm{vol} \% \mathrm{CO} \cdot \mathrm{Fe}_{2} \mathrm{O}_{3}$ 


\subsection{Magnetomechanical effect under torsional stress of iron, cobalt and nickel metals}

There are two reasons for measuring the magnetomechanical effect of these conventional transition metals under torsional stress:

(i) To explore the relation of magnetostriction and the magnetomechanical effect experimentally. Then to use the relationship to prescreen materials for magnetomechanical sensors by measuring magnetostriction. This is much simpler than measuring the magnetomechanical effect directly.

(ii) To propose proper torque sensing modes. Proper torque sensing modes can be used for evaluation of the performance of prototype magnetic torque sensor materials.

Three materials $(\mathrm{Fe}, \mathrm{Co}, \mathrm{Ni})$ were chosen for the following reasons. First of all, as metallic materials, iron, cobalt, and nickel samples are easily machined to fit the test bed while both Terfenol and ferrite are mechanically brittle and thus difficult to form. Second, the magnetostrictions of these conventional magnetic materials have been fully investigated and therefore it is easier to relate the well-documented magnetostrictive properties to the magnetomechanical effect. Since the magnetostriction of $\mathrm{Ni}$ is higher than $\mathrm{Fe}$ and $\mathrm{Co}$, most measurements were made on $\mathrm{Ni}$ samples. Magnetomechanical measurements were then made on $\mathrm{Fe}$ and Co using the best sensing mode determined from the Ni samples.

\subsection{1 $\sigma / \tau-\mathbf{H}$ process measurements}

$\sigma / \tau-\mathbf{H}$ process is the process, in which the torque was applied first to the sample, and then the field was applied. Parameters from the hysteresis loops are examined for 
torque sensing. Fig. 6-9 shows the hysteresis loops under 0 torque and $10 \mathrm{~N} \bullet \mathrm{m}$ torque of an as-machined $\mathrm{Ni}$ rod. The applied magnetic field was $\pm 2.5 \mathrm{kA} / \mathrm{m}$. Considering the demagnetizing factor, the magnetic field inside the sample was much less than $2.5 \mathrm{kA} / \mathrm{m}$. The dotted line represents the hysteresis loop without torsional stress while the solid line represents the hysteresis loop under a torque of $10 \mathrm{~N} \bullet \mathrm{m}(\mathrm{CW})$. The results show that the magnetic induction under torque is higher than the induction without torque, implying higher permeability under torque. The results also showed that the initial permeability under $10 \mathrm{~N} \bullet \mathrm{m}(\mathrm{CW})$ was significantly higher than that under 0 torque. This could be induced by an effective (stress-equivalent) field, which increased the total effective magnetic field and hence the anhysteretic magnetization under a given applied field strength. The concept of stress-effective field will be presented in chapter 7. Another observation is that the static drift of the flux meter affected the accuracy of the induction measurements (positive drift in this case). The hysteresis loop under $-10 \mathrm{~N} \cdot \mathrm{m}$ (CCW) torque showed similar behavior to that of the hysteresis loop under $10 \mathrm{~N} \bullet \mathrm{m}(\mathrm{CW})$ because only the axial component of magnetic flux was measured by the flux meter.

The hysteresis loops under 0 torque and $10 \mathrm{~N} \bullet \mathrm{m}$ torque of an as-annealed Ni rod are shown in Fig 6-10. The annealed sample has higher magnetization/permeability than asmachined nickel rods because there are fewer domain wall pinning sites in annealed materials. Applied torque increased the magnetization, as well the magnetic induction due to the effective (stress-equivalent) field. In the hysteresis loop under $10 \mathrm{~N} \bullet \mathrm{m}$, there was a negative drift of the flux meter so that the loop did not exhibit closure. 


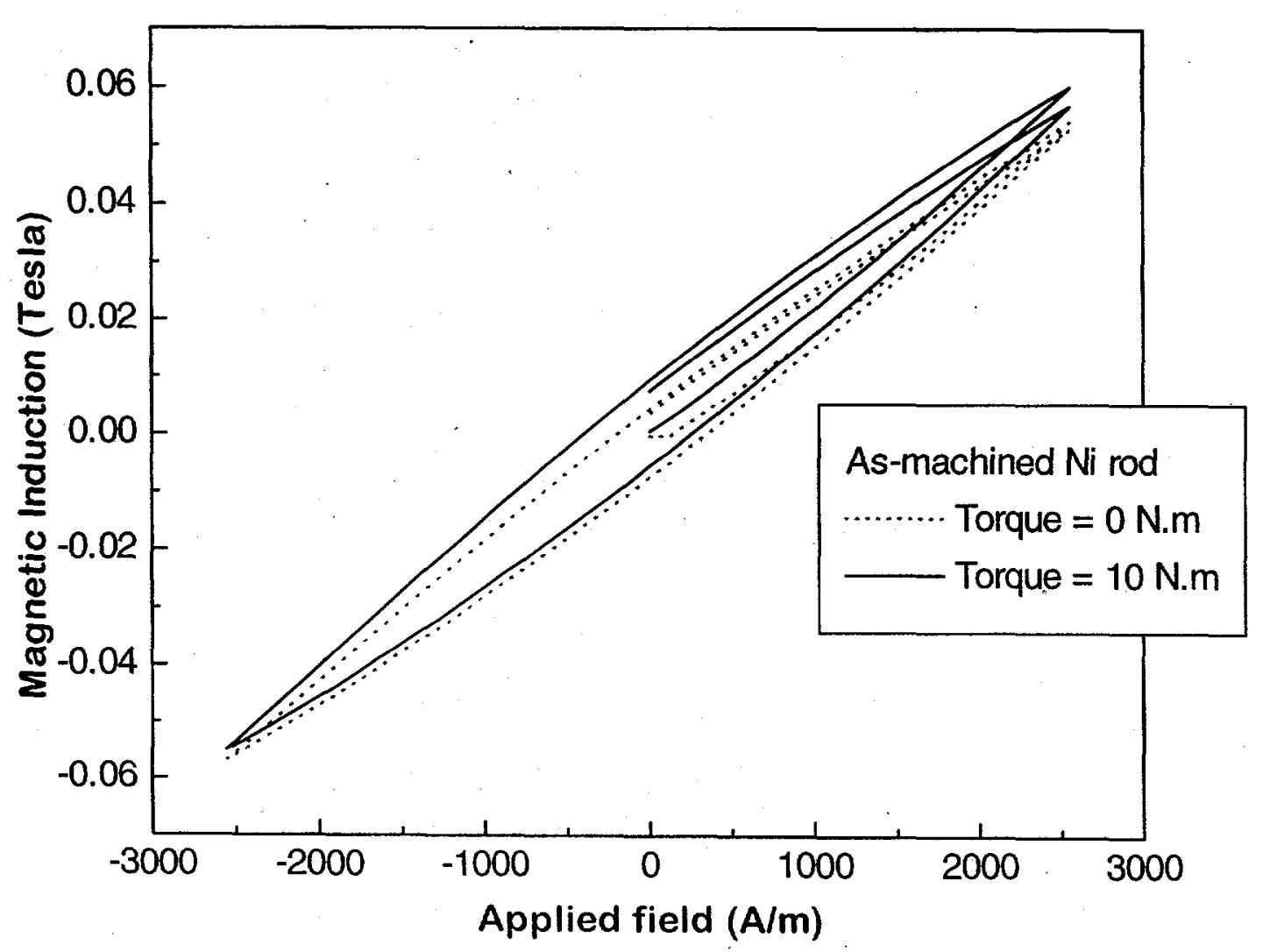

Fig. 6-9 The hysteresis loops at different torque levels of a as-machined $\mathrm{Ni}$ rod. Applied torque increased the initial permeability and magnetic induction.

In summary, in the $\sigma / \tau-\mathbf{H}$ process, torques have only a slight effect on magnetization, which is difficult to separate form the magnetic flux drift of the measurement equipment. In addition, torques in CW and CCW have the same effect on the hysteresis loop ( $\mathbf{B}$ Vs $\mathbf{H}$ ). In other words, the response of change in the hysteresis loop of the sample can not distinguish the direction of torque. Therefore, in this respect, the $\sigma / \tau-\mathbf{H}$ process is not suitable sensing mode for torque sensors. 


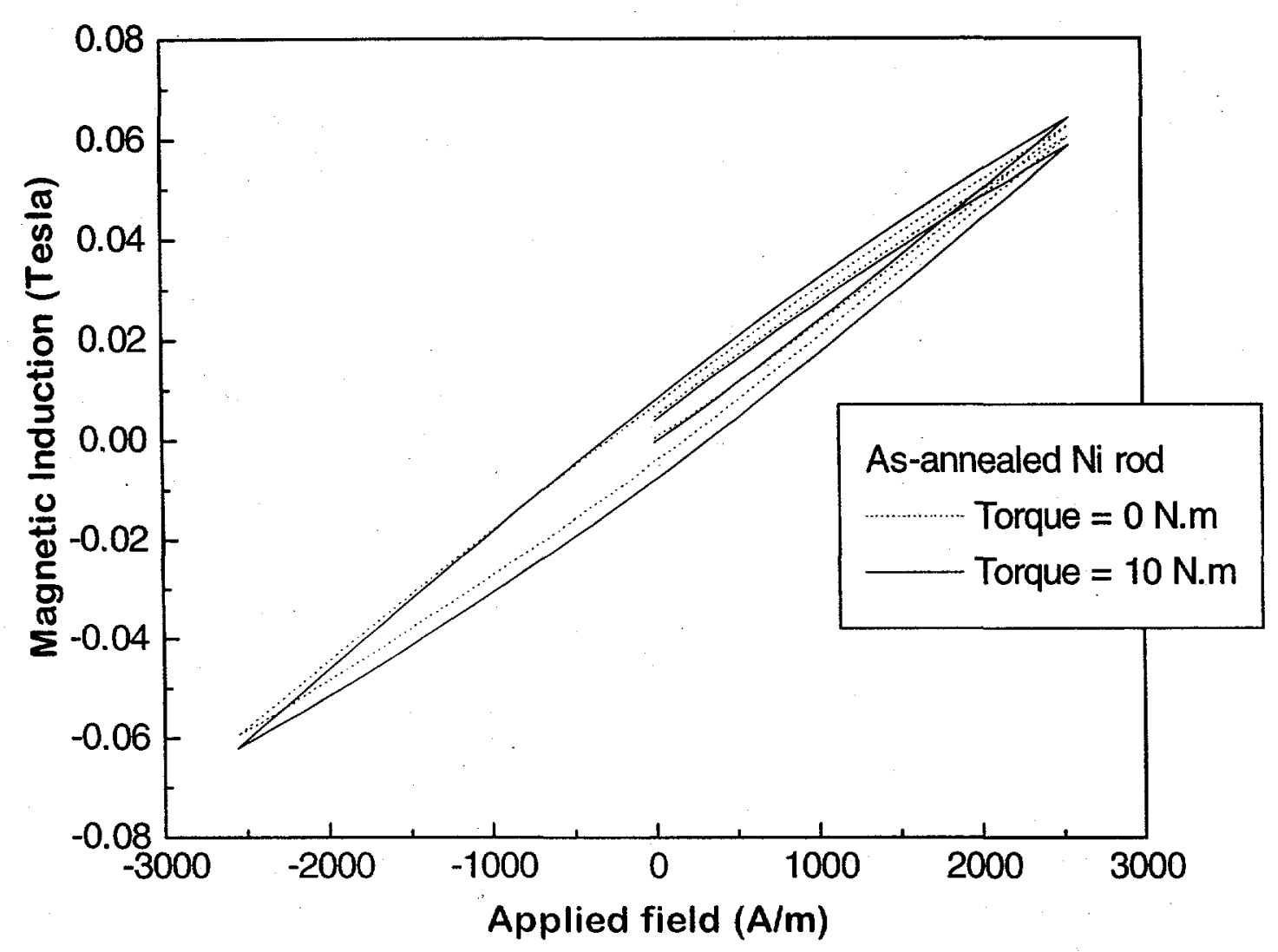

Fig. 6-10 The hysteresis loops at different torque levels of an annealed Ni rod. Annealed $\mathrm{Ni}$ has higher permeability due to fewer domain wall pinning sites. Applied torque increased magnetic induction.

\subsection{2 $\mathbf{H}-\sigma / \tau$ process measurements}

First, the surface field response with respect to applied torque was measured at an applied magnetic field of $2.5 \mathrm{kA} / \mathrm{m}$. The axial surface magnetic field was measured as a function of applied torque. The result of the as-machined rod is shown in Fig. 6-11. In this case irreversible processes dominate the change in field during the first stress cycle. It is known that the axial surface field is the vector sum of the applied field and the demagnetizing field which is proportional to magnetization and opposite to applied field. Therefore, the overall-decreasing trend of the axial magnetic field with stress cycling 
implies that the magnetization was increasing, i.e. was approaching to its anhysteretic magnetization. As the displacement between magnetization and anhysteretic magnetization decreased, the change of surface magnetic field with stress became smaller.

The results also show that the change of surface magnetic field (and also the magnetization) under the torque application process was much larger than under torque removal process. The elastic energy introduced by the applied torque has strong impact on the irreversible process governed by the law of approach. After one torque cycle, the

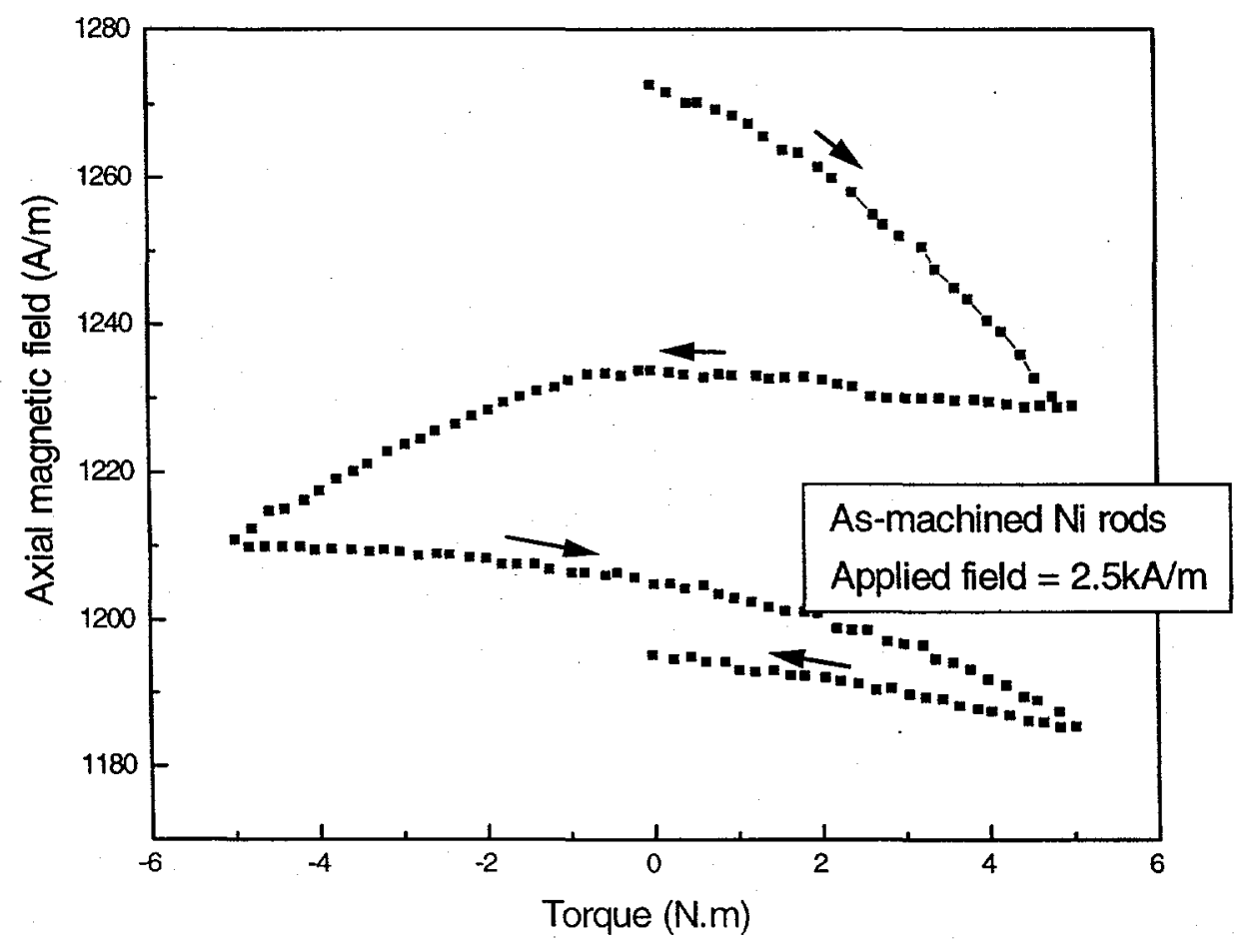

Fig. 6-11 Torque response at an applied field $2.5 \mathrm{kA} / \mathrm{m}$ of an as-machined $\mathrm{Ni}$ rod. Irreversible processes dominates the change in magnetization over the first cycle. The magnetization of the material approaches to its anhysteretic magnetization. Reversible processes appear after one torque cycle. 
existence of a reversible component of the change of field with stress became obvious when the torque was decreased from maximum $(5 \mathrm{~N} \cdot \mathrm{m})$ to zero. In this range, the irreversible component decreased significantly.

Similar results were obtained from the as-annealed sample, shown in Fig 6-12. The irreversible component dominated this process as well. The change of the magnetic field was smaller $(\sim 45 \mathrm{~A} / \mathrm{m})$ than that of the as-machined sample $(\sim 80 \mathrm{~A} / \mathrm{m})$. It was due to the smaller displacement of the magnetization from its anhysteretic value in the annealed sample. In summary, the $H-\sigma / \tau$ process under a constant applied field (at least small magnetic field) were not suitable for torque sensing either.

The torque responses were measured in the "at remanence" state. That is to say, sample magnetization was saturated in an axial applied magnetic field, the applied field was removed and then the surface magnetic fields (both axial and circumferential fields) were measured as a function of applied torque.

Annealed samples exhibited very small coercivity $(<200 \mathrm{~A} / \mathrm{m})$ which was insufficient to ensure adequate remanence and thus no useful results were obtained. The as-machined samples were tested in this sensing mode. The samples in the "as-machined" state have high residual stresses, especially surface stresses. The residual stresses imply higher dislocation density and then induce more and stronger domain wall pinning to ensure adequate coercivity and remanence for sensor applications. Applied torsional stresses can be considered as bias stresses. 


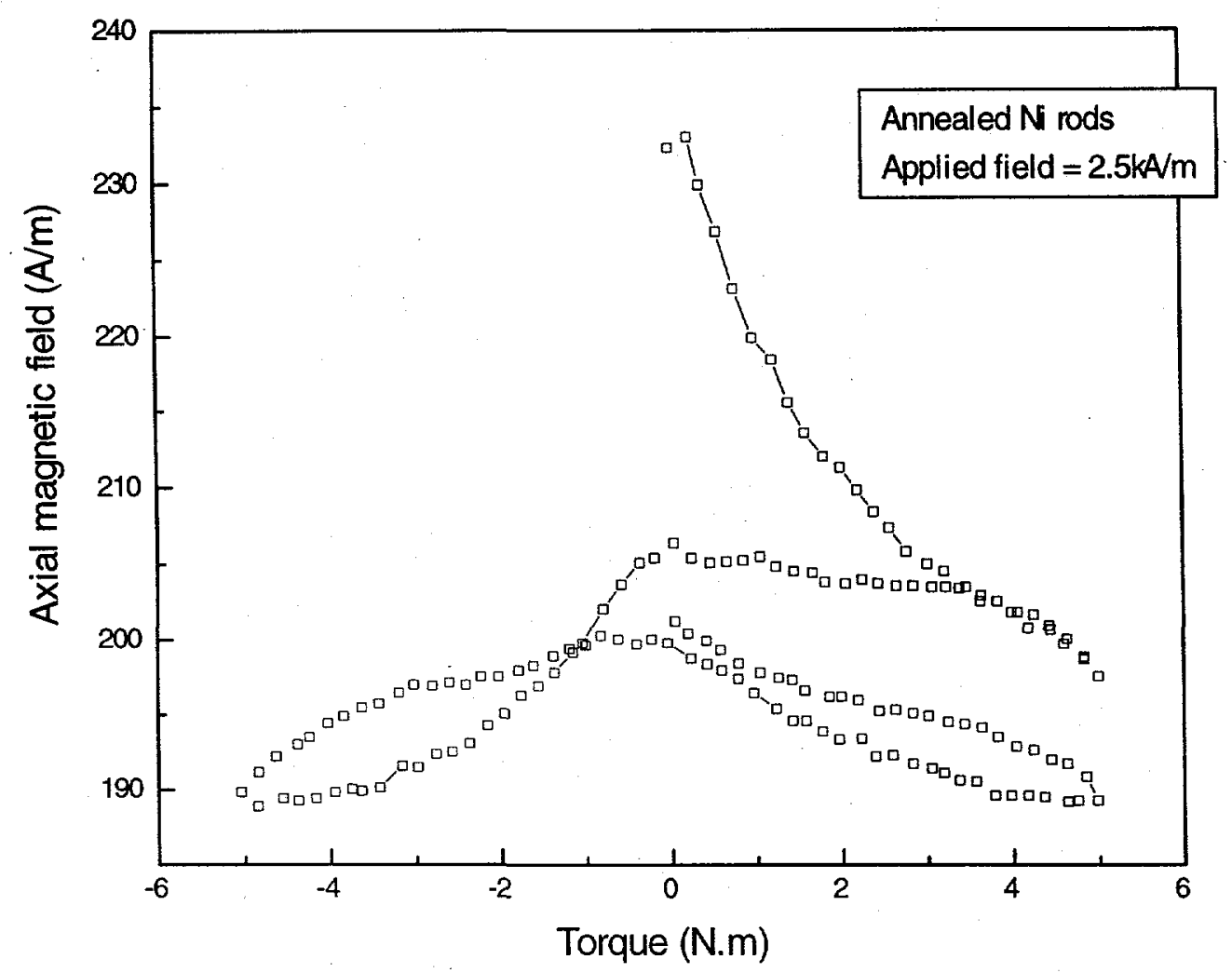

Fig. 6-12 Torque response at an applied field $2.5 \mathrm{kA} / \mathrm{m}$ of an as-annealed $\mathrm{Ni}$ rod.

It was observed that the amplitude of the axial surface field was not a single valued function of the applied torque, but rather depended on the history of torsional strain. The result for the Ni rod is shown in Fig. 6-13. The squares represent the axial field strength at $-5 \mathrm{~N} \cdot \mathrm{m}$ torque; the asterisks represent the field strength at 0 torque; and the circles represent the axial field strength at $5 \mathrm{~N} \cdot \mathrm{m}$ torque. For the same sample or same geometry, the axial surface field is proportional to the leakage magnetic flux density as well as the axial magnetization of the sample. As the number of torque cycles was increased, the amplitude of the axial field, as well as the magnetization, decreased approximately exponentially and the results became more reversible. Fitting an exponential function to the data gave a decay coefficient $\mathrm{N} 1$ of 3.3 cycles. 


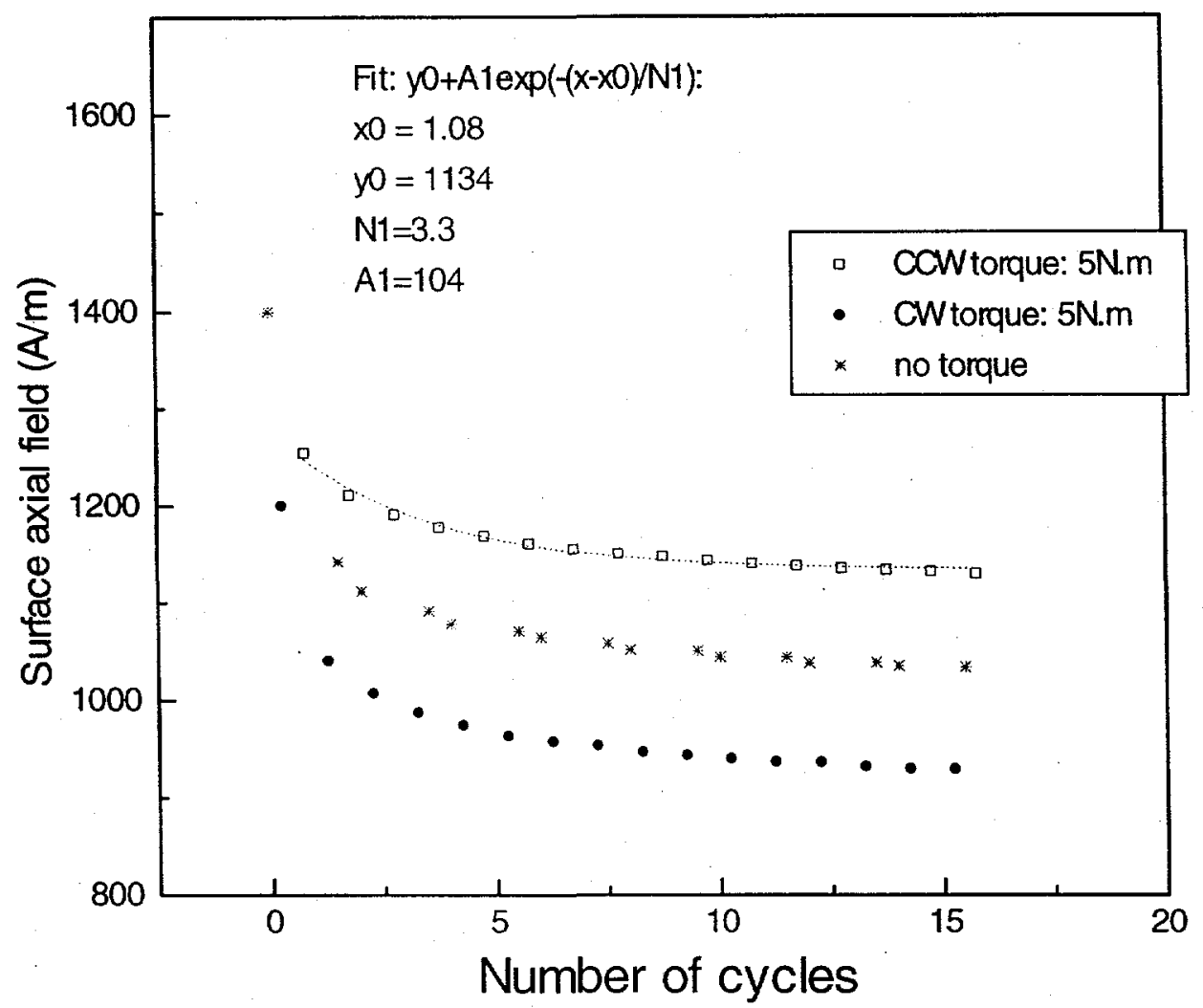

Fig. 6-13 The amplitude of the axial surface field of a Ni rod as a function of number of cycles. The squares represent the field strength at $-5 \mathrm{~N} \cdot \mathrm{m}$ torque; the asterisks represent the field strength at 0 torque; and the circles represent for the field strength at $5 \mathrm{~N} \cdot \mathrm{m}$, at different cycles, respectively. The amplitude of the axial field decreased approximately exponentially with a decay coefficient $\mathrm{N} 1$ of 3.3cycles.

However, there were still some irreversible magnetization processes taking place even after 12 torque cycles as shown in Fig. 6-14(a). These results show that there is a small amount of hysteresis in the torque cycle. Lower sensitivity but also little hysteresis was obtained by using the circumferential field from nickel after several torque cycles. The changes in circumferential field with torque after 12 cycles are shown in Fig. 6-14(b). The 
change of surface circumferential field $\mathrm{dH}_{\mathrm{cm}} / \mathrm{d} \tau$ was $18.9 \mathrm{AN}^{-1} \mathrm{~m}^{-2}$. From the directions of the axial and circumferential fields, shown in Fig. 6-10, Fig. 5-3, it was also found that, the direction of total surface field, and magnetization as well, rotated towards the compressive axis under the action of torsional stress. It is due to the negative sign of magnetostriction of nickel.

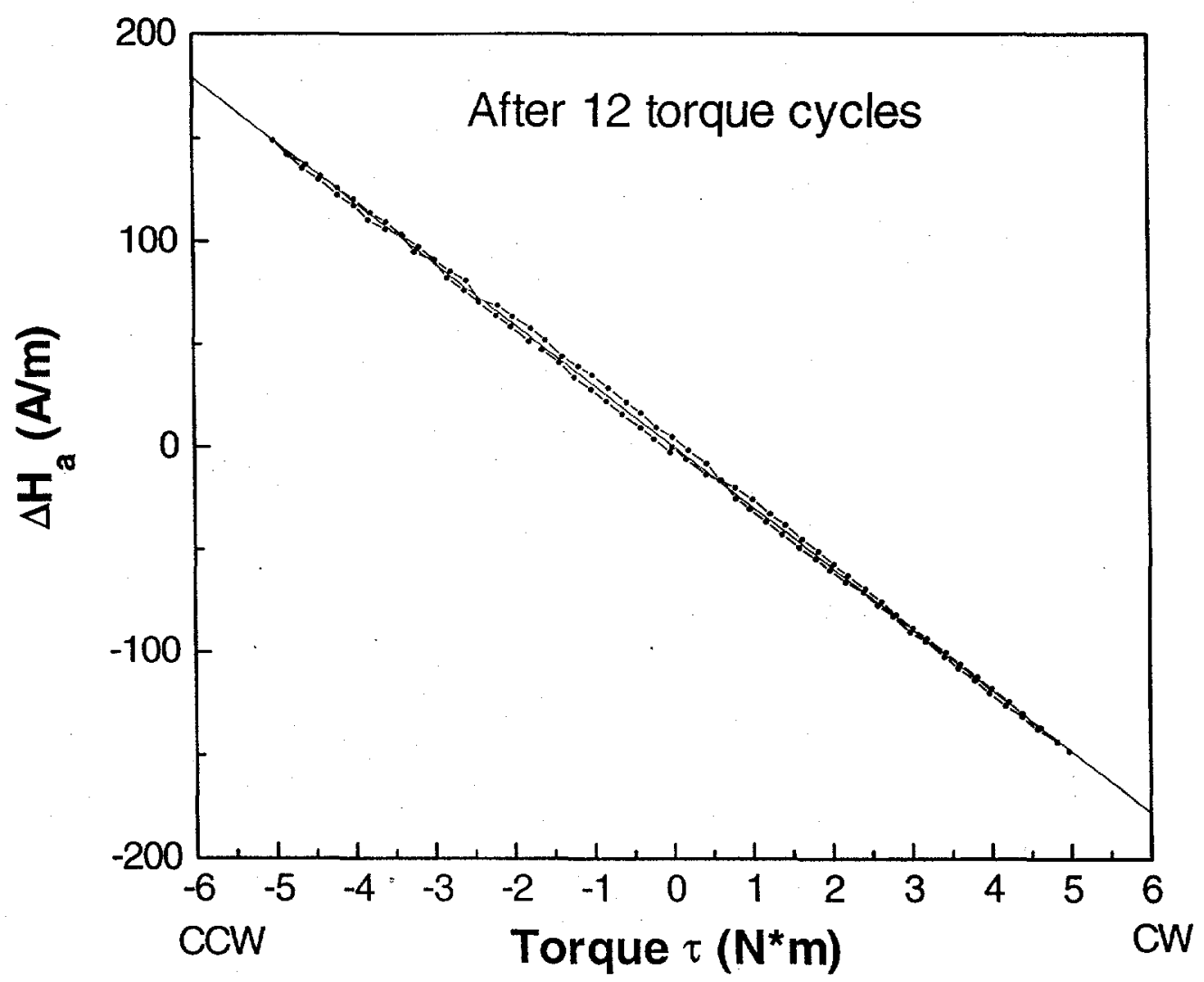

Fig. 6-14 (a) The variation of axial surface magnetic field $\mathbf{H}_{\mathbf{a}}$ of a typical Ni rod changes with applied torque after 12 torque cycles. A small hysteresis can be observed. The calculated sensitivity $\mathrm{d} \mathbf{H}_{2} / \mathrm{d} \tau$ was $29.7 \mathrm{AN}^{-1} \mathrm{~m}^{-2}$. 


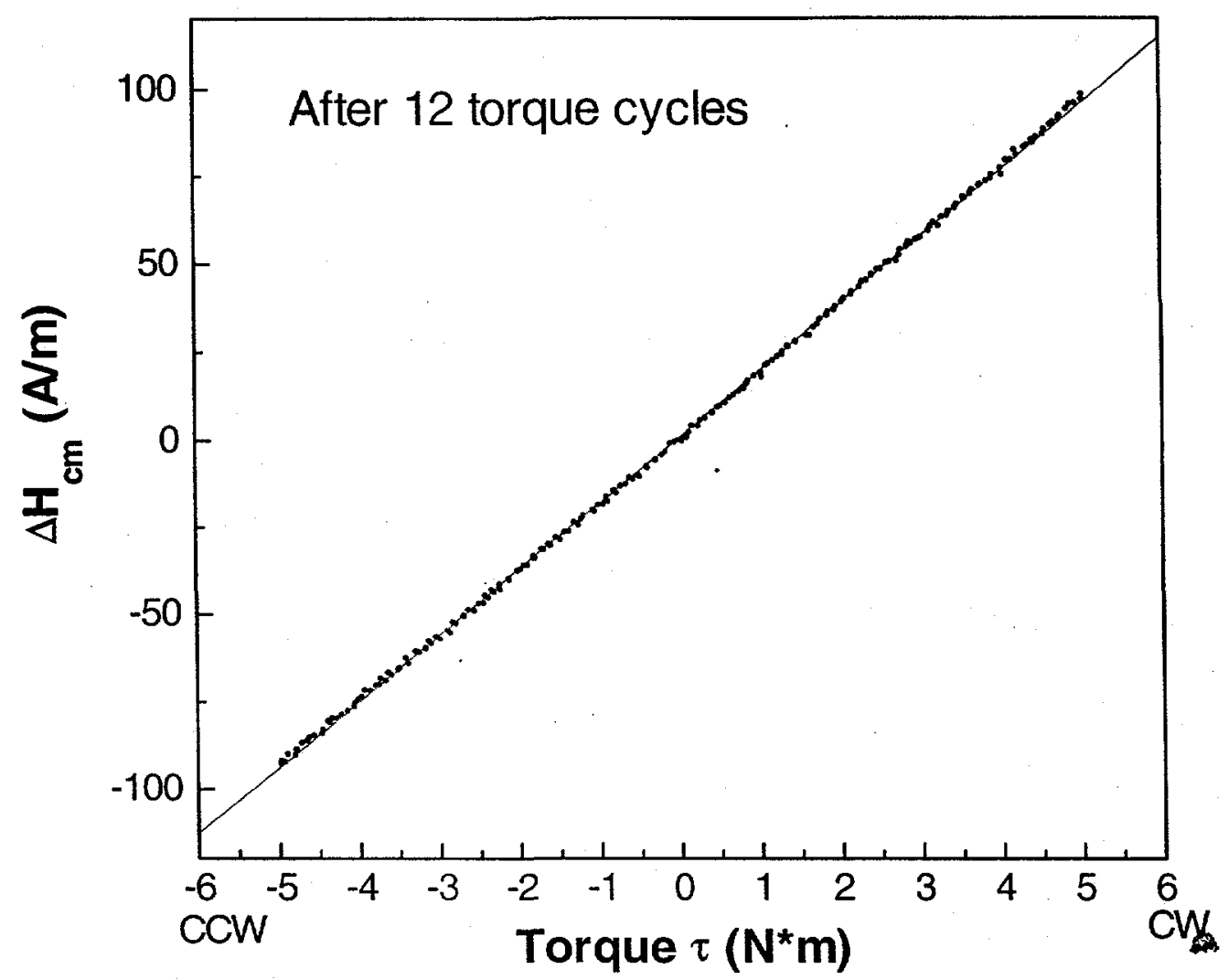

Fig. 6-14 (continued) (b) The variation of circumferential surface magnetic field $\mathbf{H}_{\mathrm{cm}}$ of the $\mathrm{Ni}$ rod changes with applied torque after 12 torque cycles. The sensitivity $\mathrm{d} \mathbf{H}_{\mathrm{cm}} / \mathrm{d} \tau$ was $18.9 \mathrm{AN}^{-1} \mathrm{~m}^{-2}$.

Cobalt and iron showed much smaller sensitivity to torsional strain. The changes of circumferential fields of $\mathrm{Fe}$ and Co rods with applied torque were $\mathrm{dH} \mathbf{H}_{\mathrm{cm}} / \mathrm{d} \tau=0.7$ and 0.2 $\mathrm{AN}^{-1} \mathrm{~m}^{-2}$, respectively.

All samples showed no sensitivity of magnetic field or magnetization to applied torque when measured in the demagnetized state. It could be considered that due to the random distribution of domains inside the sample. The effect of torsional stresses did not lead to any preferred orientation of domain magnetizations under these conditions. 
6.4.3 Discussion on the magnetomechanical effect under torsional stress

The magnetomechanical effect can be explained in terms of domain processes in magnetostrictive materials. It is known that torsional stresses on a cylinder can be considered as biaxial stresses, in which the two stress axes are perpendicular to each other and at $45^{\circ}$ to the cylinder axis. The stresses along these two axes are equal in magnitude but opposite in sign [49]. After torque is applied to the sample, domain wall motion occurs in response to the torsional stresses. The stresses have two effects on domain wall motion. From a thermodynamic viewpoint, the effect of the stresses can be considered as an effective magnetic field [38], which induces pressure on $90^{\circ}$ domain walls in magnetostrictive materials and leads to domain wall motion. This process could be thermodynamically reversible. Due to its negative magnetostriction, the magnetization of nickel would rotate towards the compressive stress direction [61], while the magnetization in iron or cobalt will rotate towards the tensile axis $[16,49]$.

However, domain wall motion can be either reversible or irreversible, depending on the strength of the domain wall pinning. If a domain wall is strongly pinned at some points but relatively free to move in-between, application of stress can cause it to bow, much like an elastic membrane, as shown in Fig. 6-15. When the torque is removed, it can return to its original position, thus giving reversible behavior. It is the reversible part of the magnetoelastic coupling that determines the sensitivity of the magnetization of the material to stress.

The second effect is that the applied stress/torque changes in the domain wall pinning energies and releases the domain wall to move towards its anhysteretic state. If 
this applied stress/torque is strong enough to release a domain wall from a pinning site and move it ahead to another location, the domain wall will not return when the stress/torque is removed, thus causing irreversible changes in magnetization, and hysteresis in the magnetomechanical response. After several torque cycles, most weak domain wall pinning will have been overcome and the domain walls will have settled into stronger pinning sites between which the domain walls could be considered as elastic membranes. The processes therefore become increasingly reversible. In this stage, magnetic anisotropy and local variations of magnetic properties mainly contribute to the hysteretic behavior shown in Fig 6-14 (a).

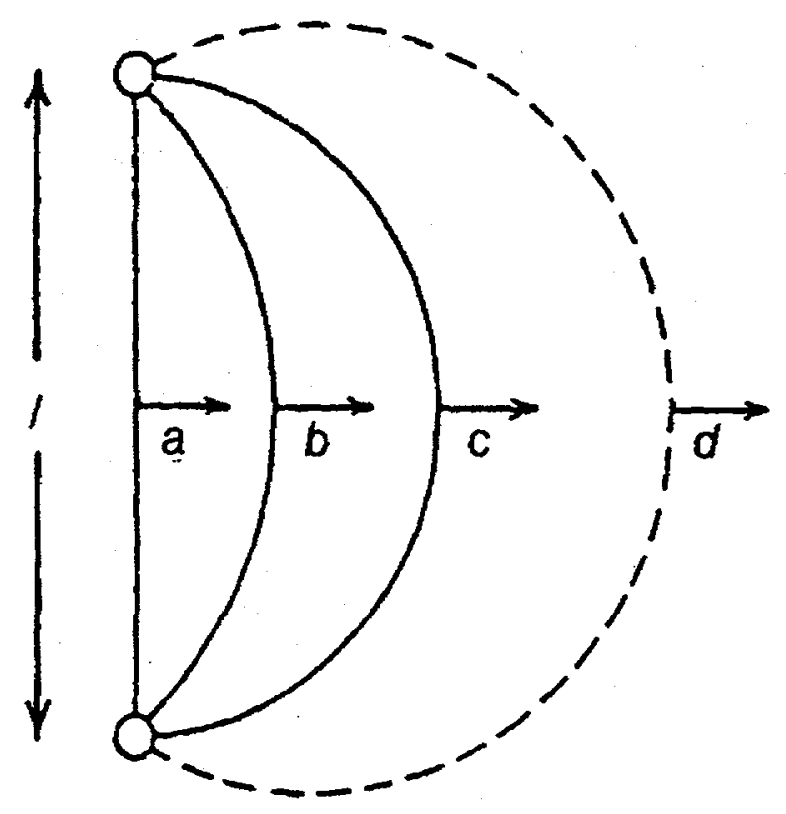

Fig.6-15 Reversible bowing of domain walls. (after Jiles [15]) 
Under reversible conditions the magnetoelastic properties can be discussed in terms of Le Chatelier's principle. For small reversible changes of magnetization, a thermodynamic relation exists, namely

$$
\left(\frac{\partial \lambda}{\partial \mathrm{H}}\right)_{\sigma}=\left(\frac{\partial \mathrm{B}}{\partial \sigma}\right)_{\mathrm{H}}=\mu_{\mathrm{o}}\left(\frac{\partial \mathrm{M}}{\partial \sigma}\right)_{\mathrm{H}}
$$

Thus a relationship between the derivative of magnetostriction with respect to field and the derivative of magnetic induction with respect to stress exists. Note that the sensitivity of magnetization to a small applied torsional stress (for example less than $10 \mathrm{~N} \cdot \mathrm{m}$ ) depends principally on the piezomagnetic coefficient $d_{33}=(\partial \lambda / \partial \mathbf{H})_{\sigma}$, rather than the saturation magnetostriction $\lambda_{s}$.

The magnetostrictions of polycrystalline iron, cobalt and nickel [19], are shown in Fig.6-16. Comparison of the current results of $\mathrm{Ni}$, $\mathrm{Co}$, and $\mathrm{Fe}$ together with their magnetostrictive properties is shown in Table 6-2. The measured magnetomechanical sensitivities of rods of $\mathrm{Ni}, \mathrm{Fe}$, and Co correlated much better with the slopes of magnetostriction $\mathrm{d} \lambda / \mathrm{dH}$ at low field, than with their saturation magnetostriction values. In view of this, a high piezomagnetic coefficient (which determines sensitivity), in conjunction with a sufficiently high saturation magnetostriction (which determines dynamic range), are the critical factors in selecting materials for magnetic torque sensors.

\subsubsection{Summary of magnetomechanical effects in metal rods}

- The magnetomechanical effect includes both reversible and irreversible components. The irreversible components change towards to the anhysteretic states of 


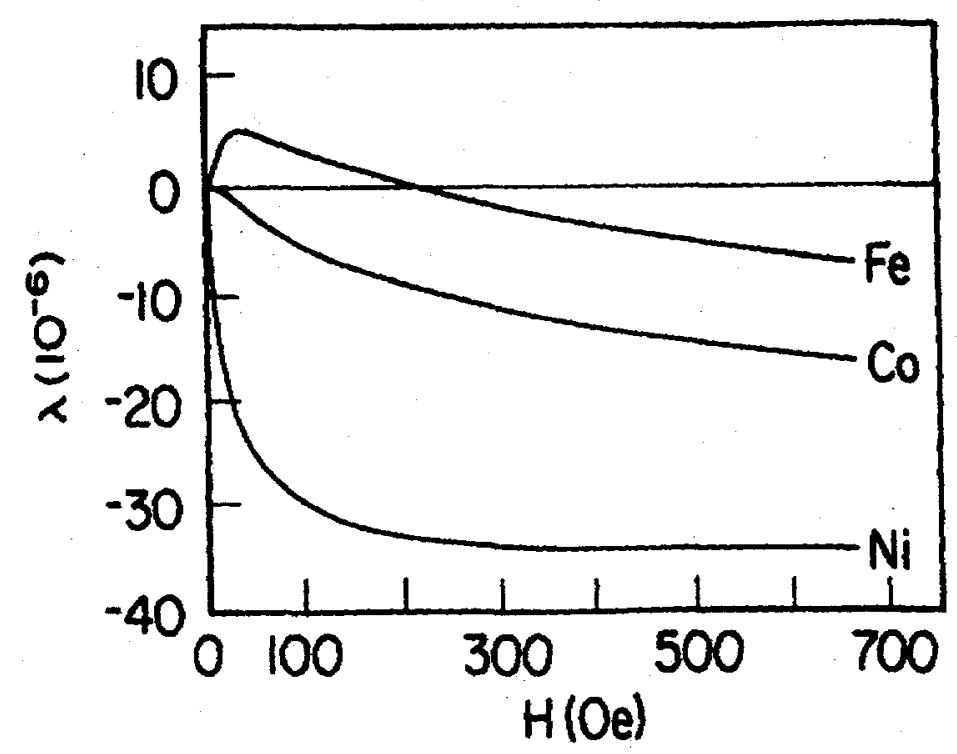

Fig.6-16 Bulk magnetostrictions of polycrystalline iron, cobalt and nickel versus applied magnetic field (after Cullity [19]).

Table 6-2 Magnetostriction and magnetomechanical effect of $\mathrm{Fe}$, $\mathrm{Co}$ and $\mathrm{Ni}$

\begin{tabular}{lccc}
\hline \hline & $\mathrm{Fe}$ & $\mathrm{Co}$ & $\mathrm{Ni}$ \\
\hline MM sensitivity & 0.7 & 0.2 & 18.9 \\
$(\mathrm{~A} / \mathrm{m}$ per N.m) & & & \\
$\lambda_{s}\left(10^{-6}\right)$ & 10 & -15 & -35 \\
$\mathrm{~d}_{33}$ at low field & Medium & Low & High \\
\hline \hline
\end{tabular}

materials and the change decreases as the displacement between magnetization and anhysteretic states decreases. The reversible component is related to the piezomagnetic coefficient.

- Sensing mode at remanence is proposed for practical magnetomechanical torque sensing which needs reversible processes. In this sensing mode, the reversible component 
dominates the process after several stress cycles.

- The magnetomechanical effect behavior has been explained on the basis of domain wall motion and pinning.

- High initial piezomagnetic coefficient, together with high saturation has been recommended for torque sensing and other magnetostrictive sensor materials.

- Reversible response was obtained from as-machined $\mathrm{Ni}$ rods. However, its repeatability is problematic for practical sensor applications.

\subsection{Magnetomechanical effects under torsional stress of Co-ferrite composites}

\subsubsection{Major loop}

Two compositions of metal-bonded Co ferrite composites were considered primary candidates for torque sensing applications. Composition of $98 \mathrm{vol} \% \mathrm{CoO} \cdot \mathrm{Fe}_{2} \mathrm{O}_{3}+2 \mathrm{vol} \%$ $\mathrm{Ag}_{0.97} \mathrm{Ni}_{0.03}$ exhibits the highest peak magnetostriction $(-230 \mathrm{ppm})$ while composition of 99 vol\% $\mathrm{CoO} \cdot \mathrm{Fe}_{2} \mathrm{O}_{3}+1$ vol\% Co exhibits the highest initial piezomagnetic coefficient $\left(2 \times 10^{-9}\right.$ $\mathrm{A}^{-1} \mathrm{~m}$ ). Several samples were sintered in the shape of a ring with different height/diameter ratio and were then brazed onto stainless steel shaft as described in chapter 5 . The performance of the former composition exhibited better stability due to its better brazability (bonding strength and uniformity) with stainless steel shafts.

The axial surface magnetic field of the prototype sensor using the method described in chapter 5 was measured as a function of applied torque. Among those prototype sensors, the sample with dimensions of $25 \mathrm{~mm}$ OD, $12.5 \mathrm{~mm} \mathrm{ID}$, and $10 \mathrm{~mm}$ high offered the best 
performance (high sensitivity and low hysteresis). The performance of this prototype sensor is shown in Fig.6-17.

In this study, magnetomechanical sensitivity $\mathrm{dH}_{\mathrm{a}} / \mathrm{d} \tau$, is defined as the slope of the linear portion of the axial field vs. torque plot and the magnetomechanical hysteresis as half of the width in $\mathrm{N} \cdot \mathrm{m}$ of the major loop when $\Delta \mathbf{H}_{\mathrm{a}}$ is zero. The magnetomechanical sensitivity was $65 \mathrm{~A} / \mathrm{m}$ per $\mathrm{N} \cdot \mathrm{m}$ with a hysteresis of $+/-0.5 \mathrm{~N} \bullet \mathrm{m}$ within a major loop of $+/-$ $10 \mathrm{~N} \cdot \mathrm{m}$. Considering the precision of the Hall probe is the $0.8 \mathrm{~A} / \mathrm{m}$. Sensitivity of $65 \mathrm{~A} / \mathrm{m}$ per $\mathrm{N} \bullet \mathrm{m}$ provides a sufficient signal to noise ratio.

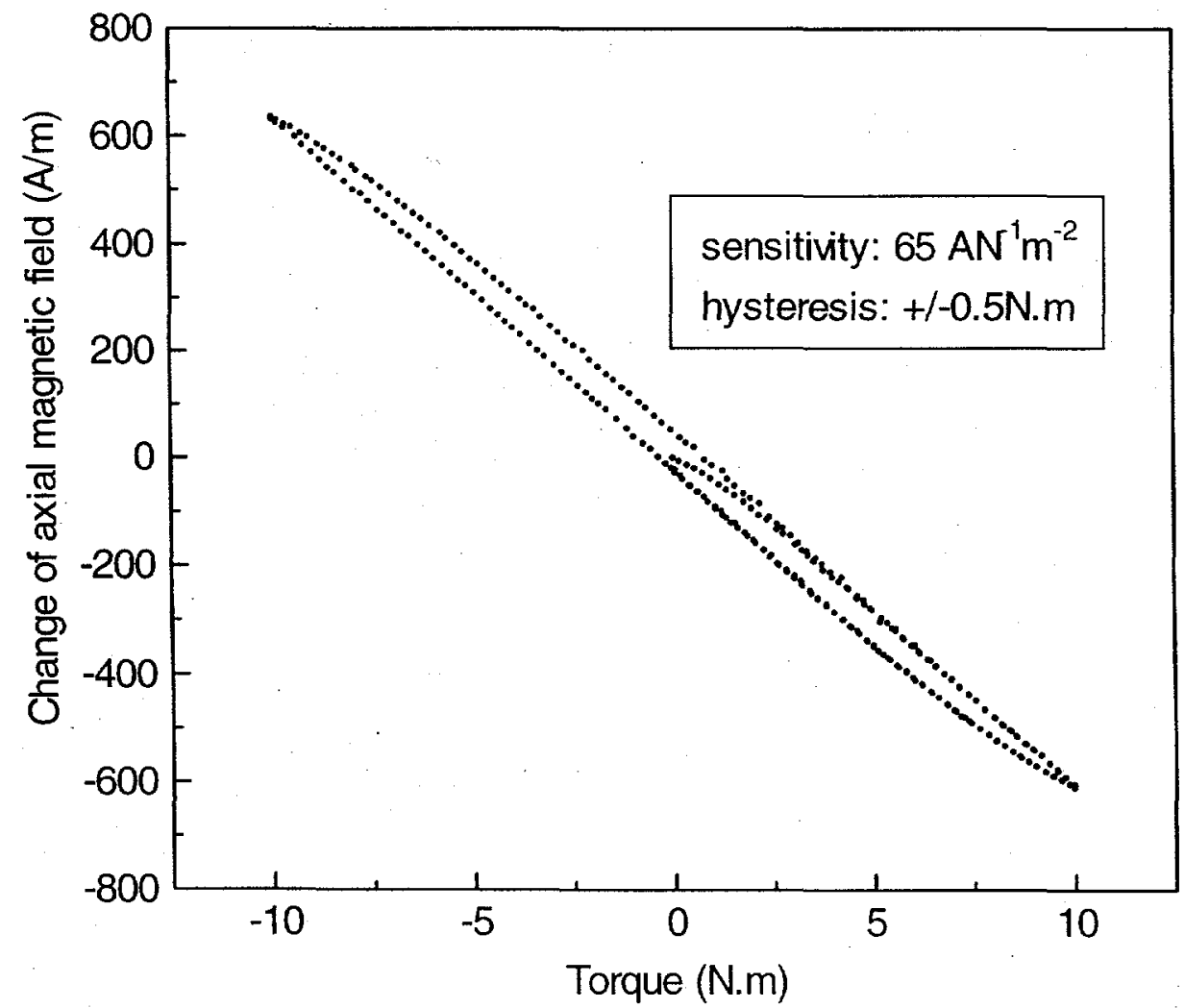

Fig. 6-17 Torque test of Co-ferrite composite ring. Composition: 2vol\% $\mathrm{Ag}_{0.97} \mathrm{Ni}_{0.03}+98 \mathrm{vol} \% \mathrm{CoO} \cdot \mathrm{Fe}_{2} \mathrm{O}_{3}$ 
Except for the magnetomechanical hysteresis, the prototype sensor showed better performance than Ni rods. Significantly it gave higher sensitivity, better repeatability, and wider torque range. The remanence of nickel depends on the residual stress which is not repeatable and its coercivity is small $(1600 \mathrm{~A} / \mathrm{m}$, as machined, $130 \mathrm{~A} / \mathrm{m}$, annealed). On the other hand, the Co ferrite composite (coercivity, $\sim 8 \mathrm{kA} / \mathrm{m}$ ) is magnetically much harder than $\mathrm{Ni}$ and thus the remanence state is much more stable. In addition, the circumferential magnetization is the preferred magnetic direction with low magnetostatic energy. Due to strong domain wall pinning in Co ferrite, domain wall unpinning seldom occurs in Co ferrites and domain wall bowing dominates the magnetization processes under torque. Thus, the magnetic field near the surface of a Co ferrite ring sample did not decay after a few torque cycles. The hysteresis of the torque response mainly comes from the anisotropy and local variations of magnetic properties.

\subsubsection{Minor loops}

For practical applications, minor loops, (i.e. arbitrary change direction of torque) occur much more frequent than a major loop, in which the torque just changes its direction at maximum torque values $( \pm 10 \mathrm{~N} \cdot \mathrm{m})$ and under which the change of torque is symmetric. A minor loop refers to any magnetic response changing with respect to any range of torque. In the present study, minor loops were chosen as a special case. The measurement started from $-10 \mathrm{~N} \cdot \mathrm{m}$ and then cycled with a step length of $1 \mathrm{~N} \cdot \mathrm{m}$ and finally ended at $10 \mathrm{~N} \cdot \mathrm{m}$. It consists of 20 minor loops (or call cycles) with step size of $1 \mathrm{~N} \bullet \mathrm{m}$. The results were shown

in Fig. 6-18. The response of surface magnetic field from the most recent torque reverse point returned to the major loop after $1 \mathrm{~N} \cdot \mathrm{m}$. 


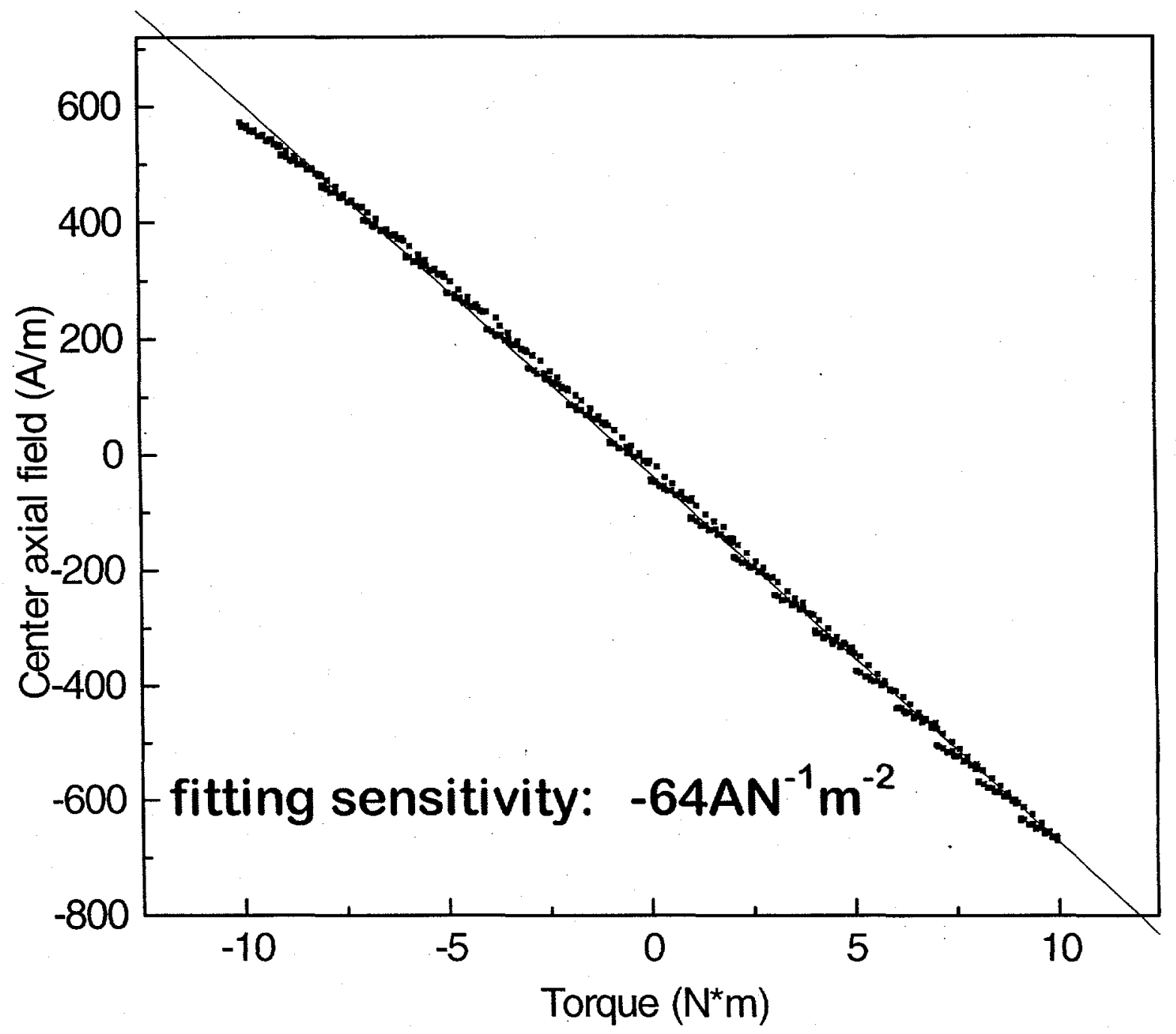

Fig. 6-18 Minor loops of the torque test. The measurements started from -10N.m and then cycled with a step length of $1 \mathrm{~N} \cdot \mathrm{m}$. After completing every cycle, the torque was increased to next cycle. The overall linear fitting sensitivity is $d \mathbf{H} / \mathrm{d} \tau$ $64 \mathrm{~A} / \mathrm{m}$ per $\mathrm{N} \cdot \mathrm{m}$. 


\subsubsection{Homogeneity}

By homogeneity is meant the uniformity of performances, including hysteresis and sensitivity at different positions of sample. Magnetic field as a function of torque at four locations equally spaced around the circumference of the prototype torque sensor was measured individually. The results are shown in Fig 6-19.
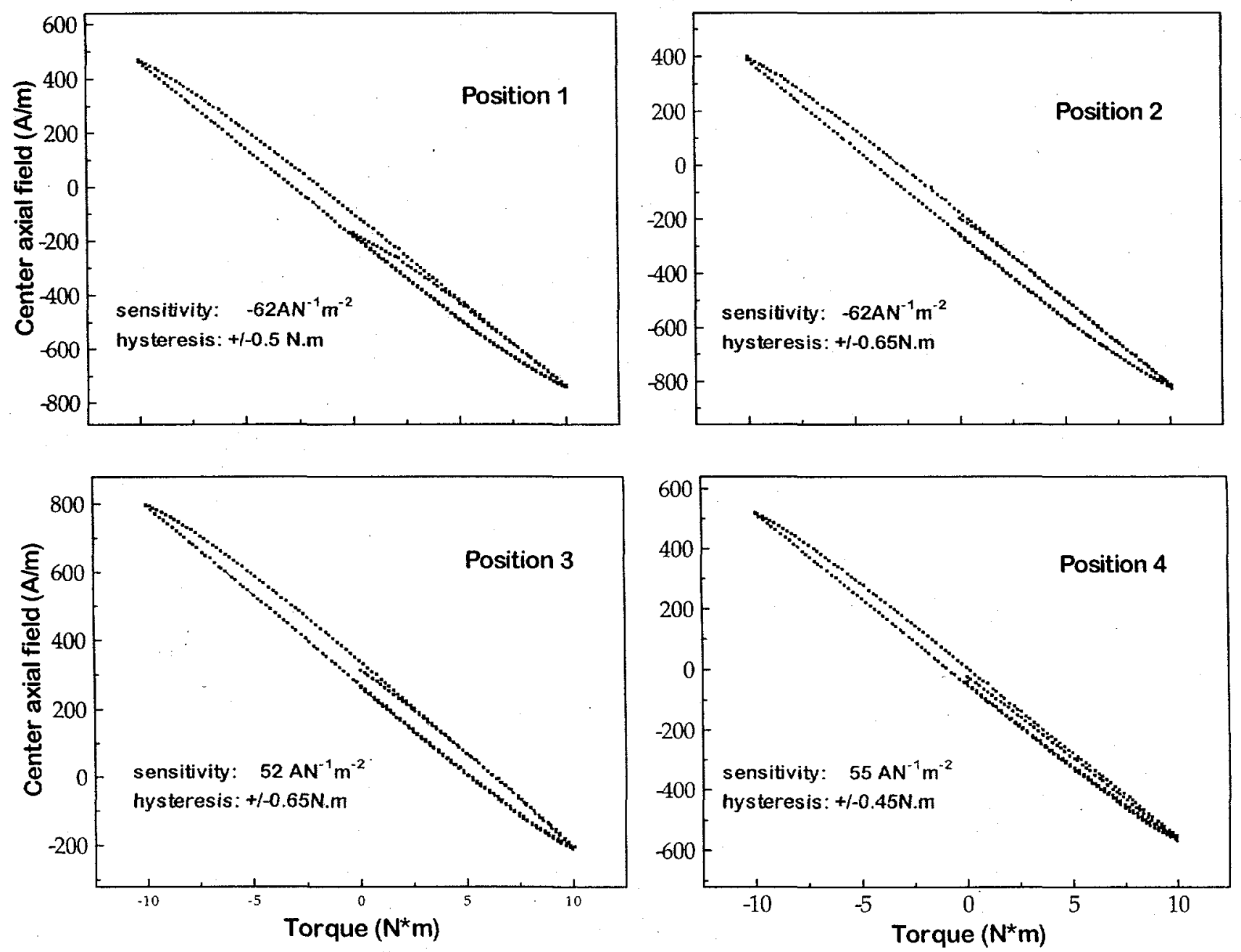

Fig. 6-19 Magnetic field as a function of torque at four locations equally spaced around the circumference of the torque sensor. The average sensitivity is $57 \mathrm{~A} / \mathrm{m}$ per $\mathrm{N} \bullet \mathrm{m}$. 
The average magnetomechanical sensitivity was calculated as $57 \mathrm{~A} / \mathrm{m}$ per $\mathrm{N} \cdot \mathrm{m}$ with a standard deviation of $\pm 5 \mathrm{~A} / \mathrm{m}$ per $\mathrm{N} \bullet \mathrm{m}$. The average magnetomechanical hysteresis is $0.56 \mathrm{~N} \cdot \mathrm{m}$ with a standard deviation of $\pm 0.09 \mathrm{~N} \cdot \mathrm{m}$. The homogeneity depends on both the uniformity of braze and uniformity of the circumferential magnetization. In order to obtain adequate homogeneity, both brazability and magnetizing procedures must be carefully controlled.

\subsection{Temperature dependence of magnetomechanical effects under torsional stress}

\subsubsection{Results}

The result of torque tests at $-37^{\circ} \mathrm{C}, 22^{\circ} \mathrm{C}$ and $80^{\circ} \mathrm{C}$ are shown in Fig. $6-20(\mathrm{a}-\mathrm{c})$. The temperature dependence of the magnetomechanical sensitivity and the magnetomechanical hysteresis over the range $-37^{\circ} \mathrm{C}$ to $90^{\circ} \mathrm{C}$ are plotted in Fig. $6-21$.

The magnetomechanical sensitivity decreased from $78 \mathrm{AN}^{-1} \mathrm{~m}^{-2}$ at $-37^{\circ} \mathrm{C}$ to $65 \mathrm{AN}$ ${ }^{1} \mathrm{~m}^{-2}$ at $22^{\circ} \mathrm{C}$ and to $34 \mathrm{AN}^{-1} \mathrm{~m}^{-2}$ at $90^{\circ} \mathrm{C}$. It decreased approximately linearly with increasing temperature as shown in Fig. 6-21. The decrease in sensitivity was $56 \%$ (on average $0.4 \%$ per deg.C). The magnetomechanical hysteresis also decreased from $\pm 1.8 \mathrm{~N} \cdot \mathrm{m}$ to \pm 0.62 $\mathrm{N} \cdot \mathrm{m}$ as the temperature increased from $-37^{\circ} \mathrm{C}$ to $22^{\circ} \mathrm{C}$ and to less than $\pm 0.1 \mathrm{~N} \cdot \mathrm{m}$ at $60^{\circ} \mathrm{C}$. Over the range $-37^{\circ} \mathrm{C}$ to $60^{\circ} \mathrm{C}$ the hysteresis decreased linearly with temperature giving a decrease of $0.02 \mathrm{~N} \cdot \mathrm{m}$ per deg.C (on average $1 \%$ per deg.C). Interestingly, the magnetomechanical hysteresis remained close to zero as the temperature increased beyond $60^{\circ} \mathrm{C}$, giving a magnetomechanical response $\mathrm{dH}_{\mathrm{a}} / \mathrm{d} \tau$ that was linear and reversible. 


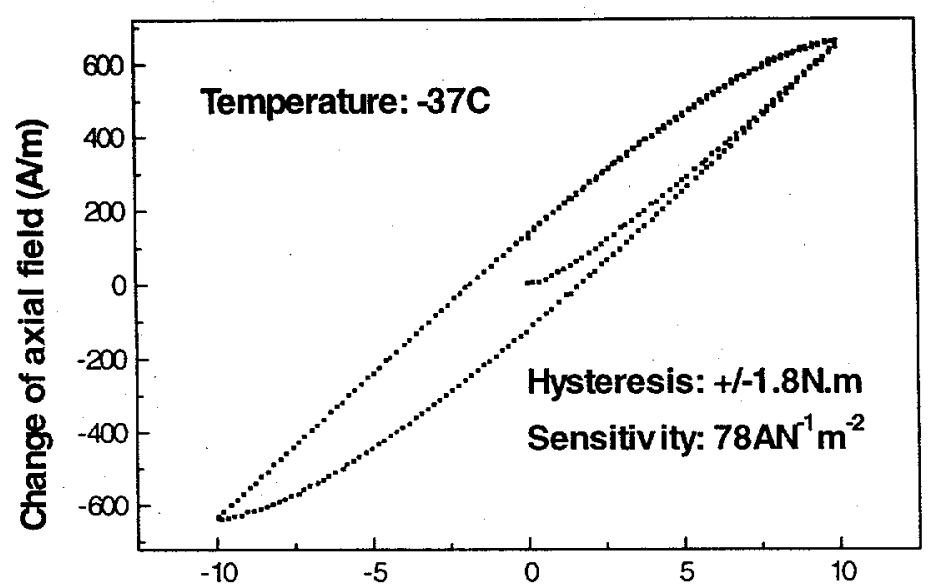

(a)

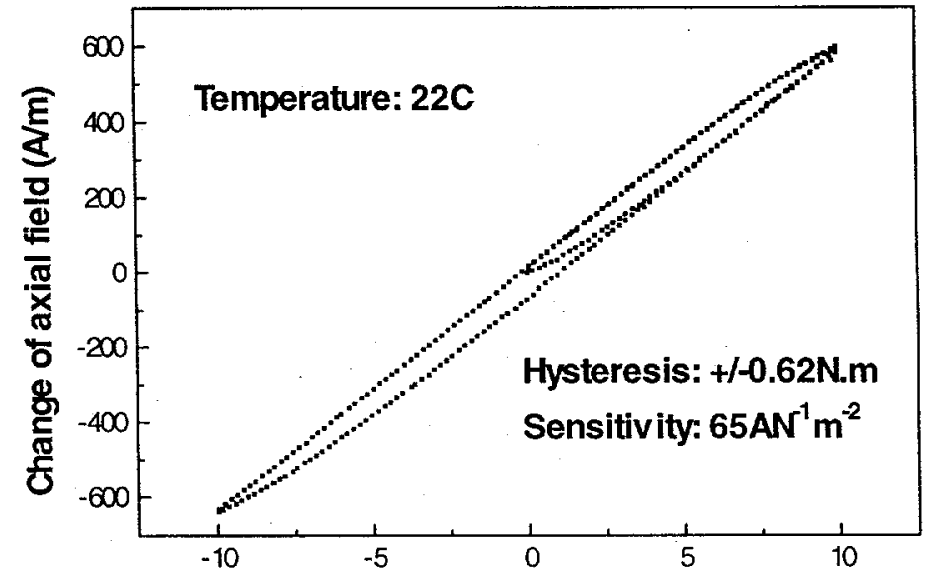

(b)

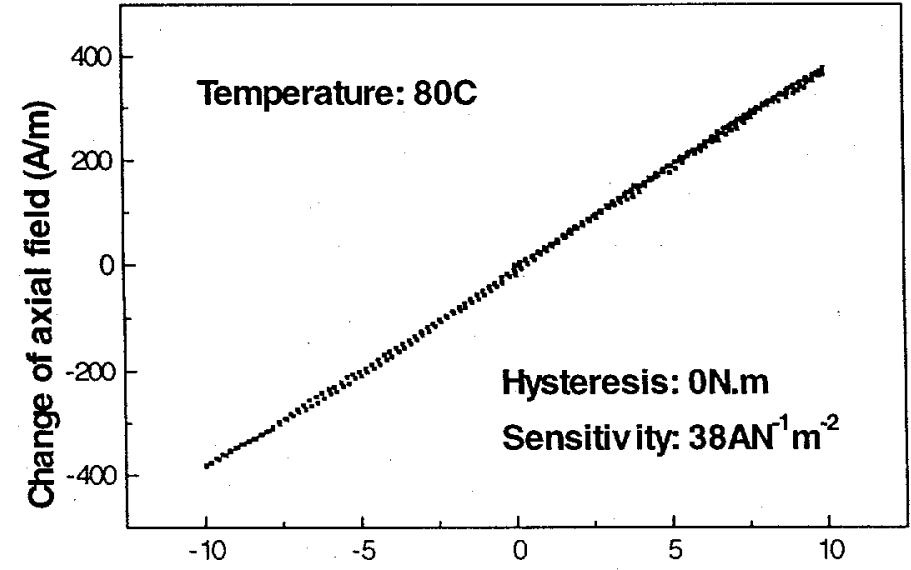

(c)

Torque $\left(\mathrm{N}^{\star} \mathrm{m}\right)$

Fig 6-20 The magnetomechanical torque responses at three typical temperatures.
(a) $-37^{\circ}$
, (b) $22^{\circ} \mathrm{C}$,
(c) $80^{\circ} \mathrm{C}$. 


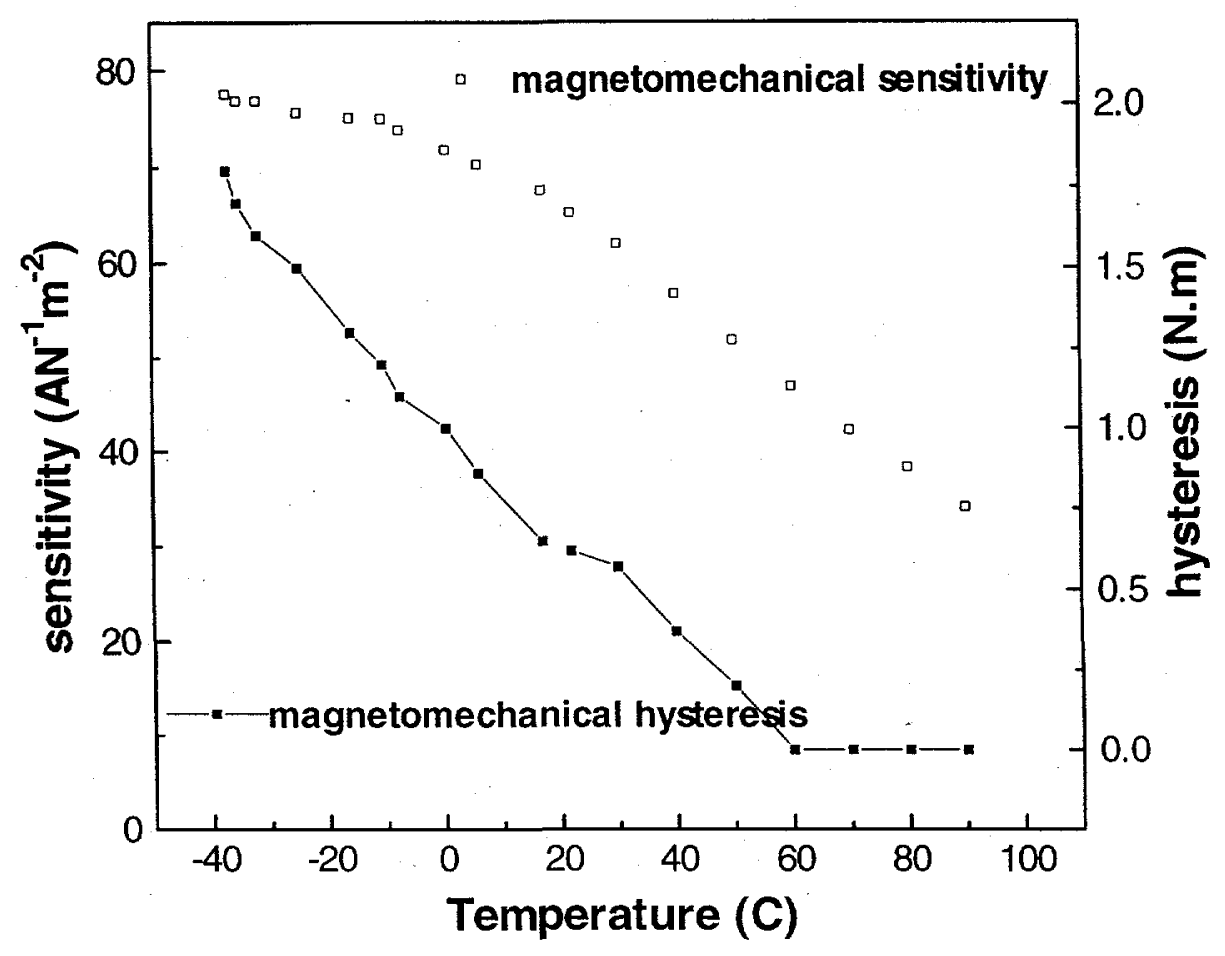

Fig. 6-21 The temperature dependence of the magnetomechanical effect of the ringshape Co-ferrite composite under torsional strain in the temperature range of $-37^{\circ} \mathrm{C}$ to $90^{\circ} \mathrm{C}$. The circles represent magnetomechanical sensitivity and the squares represent magnetomechanical hysteresis.

The torque response was also measured after the sample had been heated to $90^{\circ} \mathrm{C}$ and cooled back to $22^{\circ} \mathrm{C}$. The sensitivity and magnetomechanical hysteresis were $48 \mathrm{AN}^{-1} \mathrm{~m}^{-2}$ and $\pm 0.6 \mathrm{~N} \cdot \mathrm{m}$ respectively. As a result of thermal cycling the sensitivity had decreased by about $26 \%$, while the magnetomechanical hysteresis returned to its earlier value. These results showed some significant thermal hysteresis in the sensitivity, while the magnetomechanical hysteresis appeared to be unaffected by thermal cycling.

M-H hysteresis loops were measured over a similar temperature range as the magnetomechanical response. The coercivity, remanence, and saturation magnetization 
decreased with temperature in the range from $-40^{\circ} \mathrm{C}$ up to $100^{\circ} \mathrm{C}$, as shown in Fig. 6-22. The coercivity changed from 14.4 to $4.4 \mathrm{kA} / \mathrm{m}$ (a total of $69 \%$, or $0.5 \%$ per deg.C), the remanent magnetization changed from 70 to $26.5 \mathrm{kA} / \mathrm{m}$ (a total of $62 \%$, or $0.44 \%$ per deg.C), and the saturation magnetization changed from 445 to $387 \mathrm{kA} / \mathrm{m}$ (a total of $13 \%$, or $0.09 \%$ per deg.C).

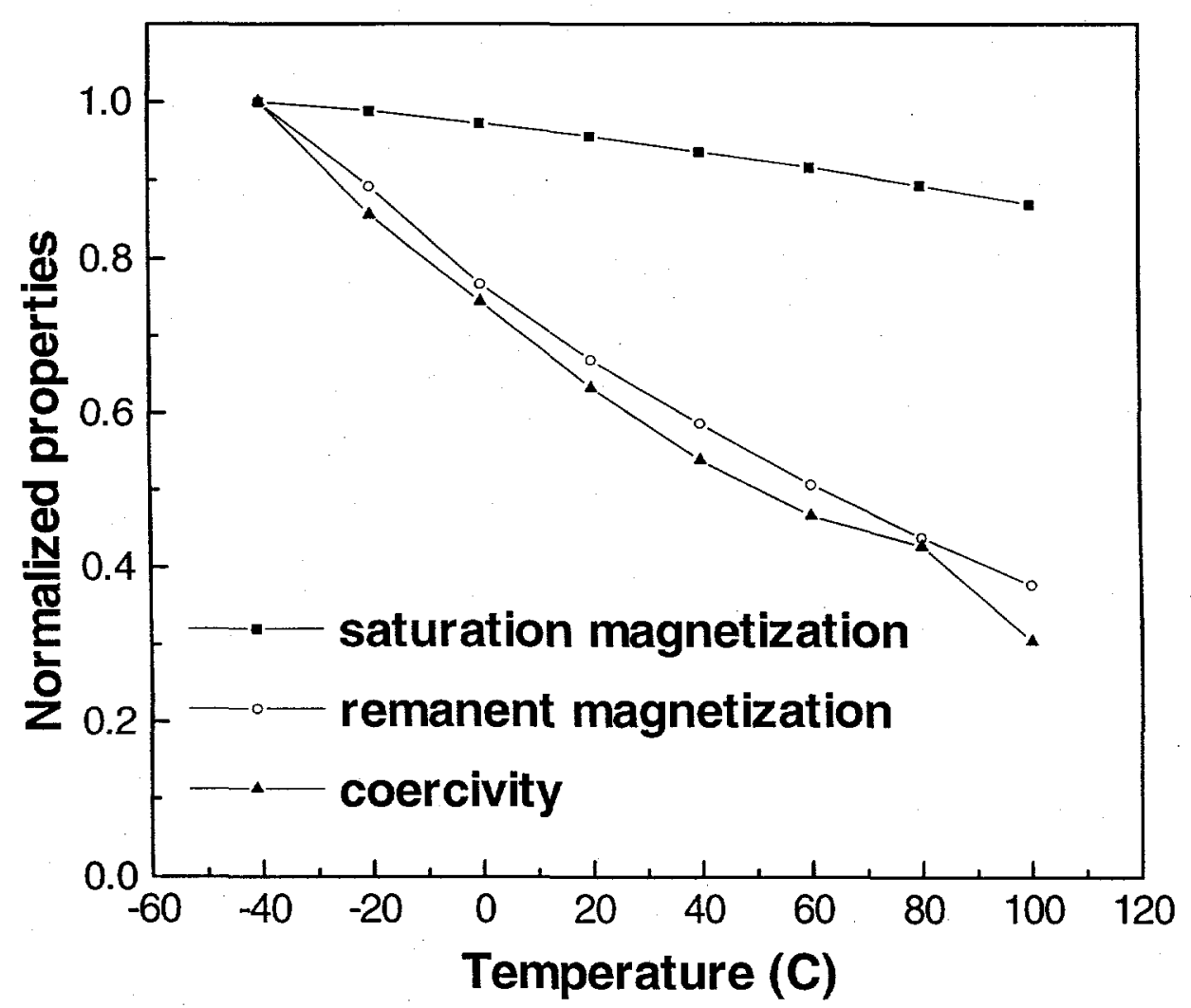

Fig. 6-22 The temperature dependence of coercivity, remanent magnetization and saturation magnetization of metal-bonded Co ferrite composite. Saturation magnetization, remanent magnetization and coercivity are normalized by their values at $-40^{\circ} \mathrm{C}$. 


\subsubsection{Discussion}

As described above, metal-bonded Co ferrite composites have been shown to have sufficient magnetoelastic response for certain types of torque sensors and other magnetostrictive sensing and actuating applications [57]. In addition, the materials have adequate mechanical properties (fracture strength $>69 \mathrm{MPa}$ ) and corrosion resistance for the application. However, little is known about the temperature dependence of the magnetomechanical response in these materials. Only a few studies have reported any results on the temperature dependence of magnetostriction or anisotropy of Co ferrites [33, $34,63]$. Due to the fact that the magnetizations of the sublattices of ferrites have opposite signs and different temperature dependence, the temperature dependence of the magnetic properties of ferrites, such as saturation magnetization and anisotropy can be complicated $[2,20]$. Metal-bonded Co ferrite composites are an even more complex systems due to the metal additives and different processing from pure Co ferrite $[20,30]$. The temperature dependence can be explained by the changes of magnetostriction, anisotropy, spontaneous magnetization and pinning of domain walls caused by the availability of increased thermal energy.

It is known that the sensitivity of the magnetomechanical effect is related to magnetostriction [38]. The temperature dependence of magnetostriction was investigated by Guillaud [34] where it was reported that Co ferrite exhibited magnetostrictions of $-270,-190$, and $-110 \mathrm{ppm}$ at $-196,-80$ and $20^{\circ} \mathrm{C}$, respectively. The magnitude of magnetostriction decreased approximately linearly from $-196^{\circ} \mathrm{C}$ to room temperature with an average decrease of magnetostriction of $0.4 \%$ per deg.C. The change of 
magnetostriction plays an important role in the temperature dependence of the magnetomechanical effect. A decrease in magnetostriction with increasing temperature indicates a decreasing magnetomechanical coupling strength, which could cause a decrease in magnetomechanical response with increasing temperature.

Although the Curie temperature of $\mathrm{Co}$ ferrite is $520^{\circ} \mathrm{C}$ [20], the saturation magnetization decreases significantly as the temperature increases in the temperature range of $-40^{\circ} \mathrm{C}$ to $100^{\circ} \mathrm{C}$, from about $490 \mathrm{kA} / \mathrm{m}$ to $427 \mathrm{kA} / \mathrm{m}$ (or 85 to $75 \mathrm{Gauss} \cdot \mathrm{cm}^{3} / \mathrm{g}$ ) [20]. Our experimental results confirmed that the metal bonded Co ferrite composite exhibits the same behavior. This effect which is due to the magnetic moments on different lattice sites changing differently with temperature caused the remanent magnetization of the ring-shape ferrite composite to decrease with temperature. This effect was reversible.

The temperature had another effect on the remanent magnetization when the temperature was raised: specifically it caused partial thermal demagnetization of the sample through release of domain walls from pinning sites. This effect was irreversible. The component of remanence decrease due to the reversible processes at higher temperatures was regained as the sample was cooled back to $22^{\circ} \mathrm{C}$, that component of remanence due to the irreversible process was not regained. Therefore, the thermal energy has only a reversible effect on the measurements at temperatures lower than ambient temperature while having both reversible and irreversible effects on the measurements at temperatures high than ambient temperature. Hence, in situations where the measurement depends on $\mathrm{M}_{\mathrm{R}}$, the sensitivity will be dependent on the thermal history since it was last magnetized. 
It is known that torsional stresses on a rod or a ring can be considered to be composed of biaxial stresses, in which the" two stress axes are perpendicular to each other and at $45^{\circ}$ to the axis of torsion [64]. The change of axial field is caused by the magnetization of the Co-ferrite ring rotating towards the compressive stress direction [57]. Therefore, the magneto-mechanical hysteresis mainly comes from the anisotropy of the ferrite composite. From the existing literature [63], it was found that the anisotropy of $\mathrm{CoO} \cdot \mathrm{Fe}_{2} \mathrm{O}_{3}$ decreased as the temperature increased. The coefficient $\mathrm{K}_{1}$ is $90 \mathrm{KJ} / \mathrm{m}^{3}$ at $90^{\circ} \mathrm{C}, 6.6 \mathrm{KJ} / \mathrm{m}^{3}$ at $200^{\circ} \mathrm{C}$, and is negligible at $280^{\circ} \mathrm{C}$ [63]. The coercivity of the metal bonded Co-ferrite composite of the present study, which would depend strongly on anisotropy, was likewise observed to decrease linearly as the temperature increased from 40 to $100^{\circ} \mathrm{C}$. Thus the temperature dependence of the magnetomechanical hysteresis is caused principally by the temperature dependence of the anisotropy. The metal additives $(\mathrm{Ag} / \mathrm{Ni})$ and the bonding treatment of the Co ferrite composite could also change the anisotropy and the temperature dependence of anisotropy. The coercivity $(\sim 8 \mathrm{kA} / \mathrm{m})$, which is mainly determined by its anisotropy of the present metal-bonded Co ferrite composite is much lower than that reported for Co ferrite at $22{ }^{\circ} \mathrm{C}(\sim 160 \mathrm{kA} / \mathrm{m})[34,59,60]$.

It is interesting to note that the magnetomechanical hysteresis effectively disappears above $60^{\circ} \mathrm{C}$ while a sufficient sensitivity still exists at that temperature to give a good signal/noise ratio (about $50 \mathrm{AN}^{-1} \mathrm{~m}^{-2}$ ). Therefore, it is possible to adjust the temperature dependent anisotropy of Co-ferrite composites by adjusting the levels of metallic additives $(\mathrm{Ag} / \mathrm{Ni} / \mathrm{Co})$ in order to obtain linear torque response at room temperature. 


\subsection{Summary of metal-bonded Co ferrite composites}

Metal-bonded Co-ferrite composites have been fabricated. These have a combination of saturation magnetostriction in excess of $200 \mathrm{ppm}$ and coercivity of about $8 \mathrm{kA} / \mathrm{m}$, which together give a high initial piezomagnetic coefficient, $(\partial \lambda / \partial \mathbf{H})_{\sigma}$ of $1.3 \times 10^{-9}$ $\mathrm{A}^{-1} \mathrm{~m}$. This is comparable to polycrystalline Terfenol [61], and much larger than Terfenolbased composites of $70 \mathrm{vol} \% \mathrm{NaPO}_{3}$, which had derivative values of typically $2 \times 10^{-10} \mathrm{~A}^{-}$

${ }^{1} \mathrm{~m}$. The magnetomechanical effect under torsional stress measured at room temperature shows that the changes of surface axial magnetic field in response to applied torque were up to $65 \mathrm{AN}^{-1} \mathrm{~m}^{-2}$, with only a small hysteresis of $\pm 0.5 \mathrm{~N} \cdot \mathrm{m}$. In addition, the metal-bonded composites are mechanically robust, corrosion-resistant, and can be brazed to shafts.

Thus metal-bonded Co-ferrite composites appear to be prime candidates for practical magnetomechanical torque sensors in the range $\pm 10 \mathrm{~N} \cdot \mathrm{m}$, and for other magnetomechanical sensing and actuating applications. The temperature dependence of the magneto-mechanical effect under torsional strain in metal-bonded Co ferrite composite was investigated. The sensitivity changed from $78 \mathrm{AN}^{-1} \mathrm{~m}^{-2}$ at $-37^{\circ} \mathrm{C}$ to $34.2 \mathrm{AN}^{-1} \mathrm{~m}^{-2}$ at $90^{\circ} \mathrm{C}$ while the magnetomechanical hysteresis decreased from $\pm 1.8 \mathrm{~N} \cdot \mathrm{m}$ at $-37^{\circ} \mathrm{C}$ to negligible above $60^{\circ} \mathrm{C}$. The effects of temperature on magnetomechanical effect under torque at remanence can be described in terms of four processes: changing magnetostriction, changing anisotropy, changing spontaneous magnetization and releasing the pinning sites of domain walls. Metal-bonded Co ferrite composites show capacity for linear magnetomechanical response to torque which can be achieved by changing the temperature dependence of anisotropy through the use of metallic additives. 


\section{MODEL OF MAGNETOSTRICTION AND MAGNETOMECHANICAL EFFECTS}

Theoretical and computational modeling is becoming increasingly important in magnetics because of the complexity of magnetism and the resurgence of applications of magnetic materials. Advances in computer technology also promote the development of numeric magnetic modeling. Preisach's model [65], Stoner-Wohlfarth's domain rotation model [66] and Jiles-Atherton [67] based initially on domain wall motion model are some examples.

\subsection{Magnetostriction model}

Magnetostriction has been extensively modeled $[26,50,68,69]$ in different respects. In the present work, a magnetostriction model has been developed [56] which is concerned with magnetostriction of composite materials, in which magnetostrictive particles are dispersed in non-magnetostrictive matrix assuming there is no magnetic coupling between the two phases.

\subsubsection{Previous study}

Herbst et al [70] have studied $\mathrm{SmFe}_{2} / \mathrm{Fe}$ and $\mathrm{SmFe}_{2} / \mathrm{Al}$ composites and described a model to predict the magnetostriction of composite materials. This model is a solution of the equation for elastic equilibrium considering an elastically isotropic magnetostrictive sphere embedded in an infinite, elastically isotropic, non-magnetostrictive medium. It 
assumed a force balance at the boundary of the particle and the matrix and predicted that the magnetostriction of the composite depends only on the fill fraction of particles and Poisson's ratio $\sigma$ of the matrix. The normalized magnetostriction was written as

$$
\lambda^{*}=\frac{f}{1-1.25 \sigma}\left(1.1538-1.2628 \sigma-0.2122 f^{\frac{2}{3}}\right)
$$

where $\mathrm{f}$ is the fill fraction and $\sigma$ is the Poisson's ratio of the matrix. Poisson's ratio for isotropic materials is 0.25 while the maximum value for which there is no net volume change is 0.5 . For many metal and alloys, the value of Poisson's ratio ranges between 0.25-0.35 [71]. The modeled results with three different values of Poisson's ratios are plotted in Fig. 7-1. The figure shows that for different composite materials with the same fill fraction of magnetostrictive phases, the predicted magnetostrictions are very close. In other words, the Poisson's ratio of matrix material has only a slight effect on the magnetostriction of composite materials. This model does not agree with the experimental results obtained in the present study. The measured saturation magnetostriction with $10 \mathrm{vol} \%$ Terfenol changed from a value of $90 \mathrm{ppm}$ to a value of $15 \mathrm{ppm}$ in different materials. It was also noticed, in the same paper [70], the experimental magnetostriction of $\mathrm{Al}$ based samples is only half of the result predicted by this model. However, Herbst's model does not explain the role of the matrix material in magnetostrictive composites. Recently, Nan [71] has also proposed an analytical model for the effective magnetostriction of magnetostrictive composites based on Green's function technique. Although it gives better agreement with experiment than Herbst's model, the effect of elastic modulus was not taken account. 


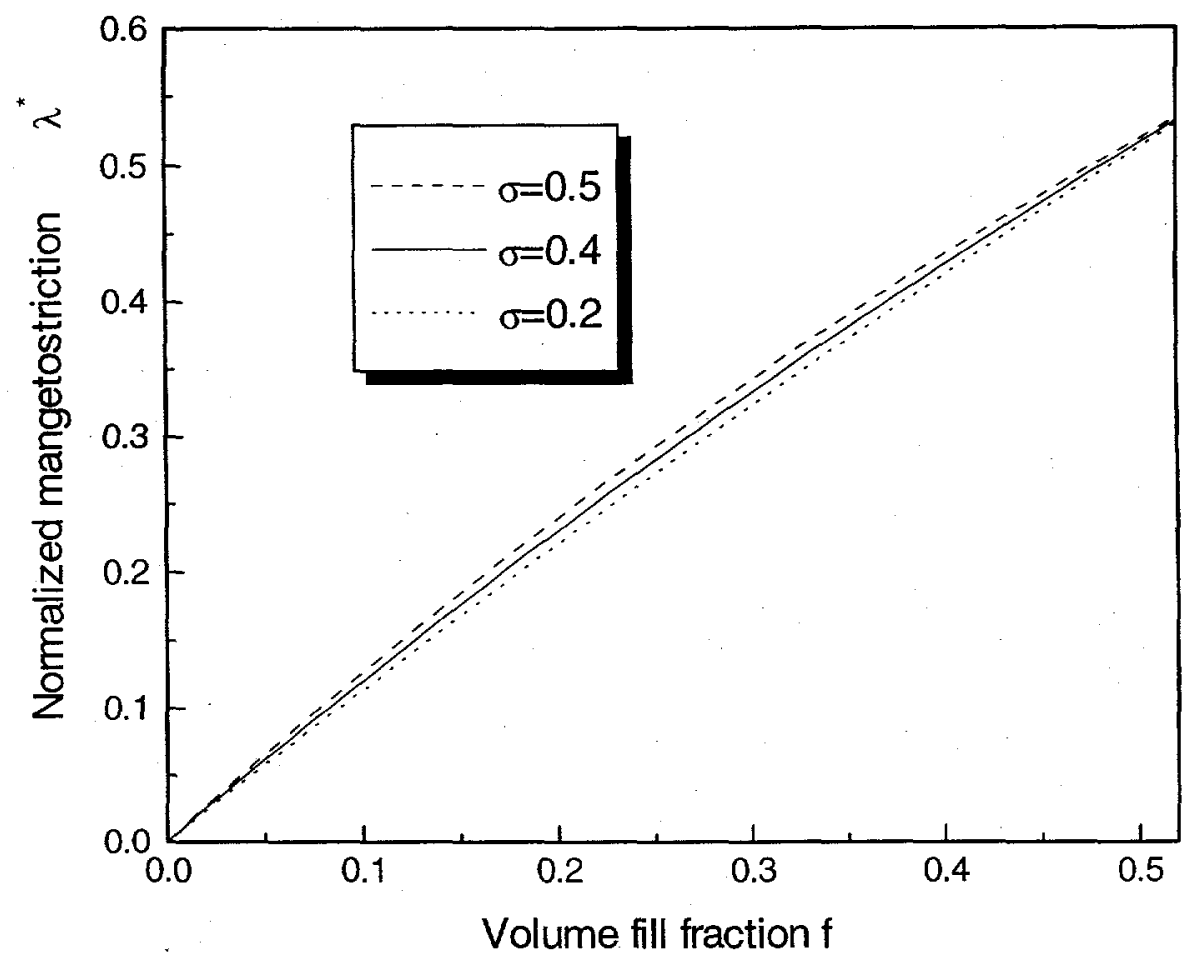

Fig. 7-1 Normalized magnetostriction of magnetostrictive composites modeled by Herbst et al [70].

\subsubsection{Effect of elastic modulus on composite magnetostrictive materials}

In the present study, a model based on the effect of elastic modulus on composite magnetostrictive materials has developed. This magnetostriction model [56] is based on that developed for the elastic modulus of fiber and particle-reinforced composites [71]. This predicts that the elastic modulus of a particle-reinforced composite should fall between an upper bound, which is based on an assumption of uniform strain, and a lower bound which is based on an assumption of uniform stress [71]. The equations for the 
elastic modulus in the two limiting cases are given in the following sections. The same approach was used to develop a model of magnetostriction.

\subsubsection{Uniform strain case}

If the strain is the same throughout the composite then the integrated stress across the entire composite can be expressed as the product of the strain and the overall elastic modulus of the composite, $\mathrm{E}_{\mathrm{c}}$

$$
\varepsilon \mathrm{E}_{\mathrm{c}}=\varepsilon \mathrm{E}_{\mathrm{t}} \mathrm{V}_{\mathrm{i}}+\varepsilon \mathrm{E}_{\mathrm{m} 1} \mathrm{~V}_{\mathrm{m} 1}+\varepsilon \mathrm{E}_{\mathrm{m} 2} \mathrm{~V}_{\mathrm{m} 2}+\ldots
$$

where $\varepsilon=$ strain, $\mathrm{V}=$ volume fraction, $\mathrm{E}=$ elastic modulus. The subscript $\mathrm{c}$ implies composite, $\mathrm{m}$ implies matrix, $\mathrm{t}$ implies the magnetostrictive phase. Canceling the strain throughout, the resulting upper bound of the elastic modulus of the composite is given by

$$
E_{c}=E_{t} V_{t}+E_{m 1} V_{m 1}+E_{m 2} V_{m 2}+\ldots
$$

\subsubsection{Uniform stress case}

If the stress throughout the material is uniform then from Hooke's law

$$
\sigma=\mathrm{E} \varepsilon
$$

where $\sigma=$ stress. It follows that uniform stress implies the product E $\varepsilon$ must be the same for each component of the composite, and therefore

$$
\sigma=\mathrm{E}_{1} \varepsilon_{\mathrm{i}}=\mathrm{E}_{\mathrm{m} 1} \varepsilon_{\mathrm{m} 1}=\mathrm{E}_{\mathrm{m} 2} \varepsilon_{\mathrm{m} 2}=\ldots
$$


The bulk or average strain of the entire composite is given by the sum of the strains weighted in proportion to their volume fractions

$$
\varepsilon_{\mathrm{c}}=\varepsilon_{\mathrm{t}} \mathrm{V}_{\mathrm{t}}+\varepsilon_{\mathrm{m} 1} \mathrm{~V}_{\mathrm{m} 1}+\varepsilon_{\mathrm{m} 2} \mathrm{~V}_{\mathrm{m} 2}+\ldots
$$

Substituting (7-5) into (7-6) gives,

$$
\varepsilon_{c}=\frac{\sigma}{E_{c}}=\sigma \frac{V_{t}}{E_{1}}+\sigma \frac{V_{m 1}}{E_{m 1}}+\sigma \frac{V_{m 2}}{E_{m 2}}+\ldots
$$

and canceling the stress throughout, gives a lower bound for the elastic modulus of the composite

$$
\mathrm{E}_{\mathrm{c}}=\frac{1}{\frac{\mathrm{V}_{\mathrm{t}}}{\mathrm{E}_{\mathrm{t}}}+\frac{\mathrm{V}_{\mathrm{m} 1}}{\mathrm{E}_{\mathrm{m} 1}}+\frac{\mathrm{V}_{\mathrm{m} 2}}{\mathrm{E}_{\mathrm{m} 2}}+\ldots}
$$

The above equations (7-3) and (7-8) for elastic modulus of composites are well known [71]. They correspond two examples, longitudinal and transverse loading on fiberreinforced composites and were considered as the lower bound and upper bound of the elastic modulus of particle-reinforced composites. Similar concepts can be applied to the magnetostriction of composite materials.

\subsubsection{Magnetostriction limits}

In the magnetostriction model developed in the present work, it is assumed that the stress generated in the composite is caused by the magnetostrictive phase and that the overall stress in the composite when it is magnetically saturated is the product of the 
volume fraction of magnetostrictive phase and the stress exerted by it. Under these conditions the following equation is obtained

$$
\mathrm{E}_{\mathrm{c}} \lambda_{\mathrm{s}}=\mathrm{E}_{\mathrm{t}} \lambda_{\mathrm{t}}^{\mathrm{s}} \mathrm{V}
$$

where $\lambda_{s}$ is the saturation magnetostriction of the composite, and $\lambda_{t}^{s}$ is the saturation magnetostriction of the magnetostrictive phase alone. Substituting for the upper bound and the lower bound of the elastic modulus from equations (7-3) and (7-8) respectively, the limiting values of the saturation magnetostriction are given by:

$$
\begin{aligned}
& \lambda_{\mathrm{se}}=\frac{\mathrm{E}_{\mathrm{t}} \lambda_{\mathrm{t}}^{s} \mathrm{~V}_{\mathrm{t}}}{\mathrm{E}_{\mathrm{t}} \mathrm{V}_{\mathrm{t}}+\mathrm{E}_{\mathrm{m} 1} \mathrm{~V}_{\mathrm{m} 1}+\mathrm{E}_{\mathrm{m} 2} \mathrm{~V}_{\mathrm{m} 2}+\ldots} \\
& \lambda_{\mathrm{s} \sigma}=\mathrm{E}_{\mathrm{t}} \lambda_{1}^{s} \mathrm{~V}_{\mathrm{t}}\left(\frac{\mathrm{V}_{1}}{\mathrm{E}_{1}}+\frac{\mathrm{V}_{\mathrm{m} 1}}{\mathrm{E}_{\mathrm{m} 1}}+\frac{\mathrm{V}_{\mathrm{m} 2}}{\mathrm{E}_{\mathrm{m} 2}}+\ldots\right)
\end{aligned}
$$

Generally, the elastic modulus of a composite lies between these limits [72] and therefore, the saturation magnetostrictions of composites should fall between the values of $\lambda_{s \varepsilon}$ and $\lambda_{\text {so }}$ given by equations (7-10) and (7-11).

\subsubsection{Comparison of experimental and modeled results}

Magnetostriction curves of two series of samples were measured in order to test these theoretical predictions. The first was a series of samples with a fixed volume fraction of the same magnetostrictive phase in matrix materials with different elastic moduli. The second was a series of samples with different volume fractions of the magnetostrictive phase in the same matrix material. This allowed the effects of the elastic 
modulus of the matrix and volume fraction of the magnetostrictive phase to be studied independently. The value of the elastic moduli of the matrix materials, measured values of saturation magnetostrictions and calculated magnetostrictions based on the upper bound and lower bound model equations are given in Table 7-1.

Table 7-1 Measured and predicted saturation magnetostrictions of composites

\begin{tabular}{|c|c|c|c|c|c|c|}
\hline \multirow[b]{2}{*}{ Sample } & \multirow{2}{*}{$\begin{array}{c}\text { Vol\% } \\
\text { Terfenol+matrix }\end{array}$} & \multirow{2}{*}{$\begin{array}{l}\text { Measured saturation } \\
\text { magnetostriction } \lambda_{\mathrm{e}} \\
(\mathrm{ppm})\end{array}$} & \multicolumn{2}{|c|}{ Uniform strain } & \multicolumn{2}{|c|}{ Uniform stress } \\
\hline & & & $\begin{array}{c}\mathrm{E}_{\mathrm{m}} \\
(\mathrm{GPa})\end{array}$ & $\begin{array}{c}\lambda_{u} \\
(\mathrm{ppm})\end{array}$ & $\begin{array}{c}\mathrm{E}_{\mathrm{m}} \\
(\mathrm{GPa})\end{array}$ & $\begin{array}{c}\lambda_{1} \\
(\mathrm{ppm})\end{array}$ \\
\hline $\bar{A}$ & $10 / 90 \mathrm{~T}+\mathrm{F}$ & 15 & 175 & 18 & 123 & 26 \\
\hline B & $10 / 90 \mathrm{~T}+\mathrm{C}$ & 25 & 112 & 27 & 96 & 33 \\
\hline $\mathrm{C}$ & $10 / 90 \mathrm{~T}+\mathrm{A}$ & 55 & 66 & 45 & 64 & 48 \\
\hline $\mathrm{D}$ & $10 / 90 \mathrm{~T}+\mathrm{E}$ & 90 & 38 & 79 & 35 & 79 \\
\hline E1 & $10 / 90 \mathrm{~T}+\mathrm{G}$ & 60 & 50 & 58 & 50 & 60. \\
\hline E2 & $30 / 70 \mathrm{~T}+\mathrm{G}$ & 200 & 50 & 190 & 50 & 201 \\
\hline E3 & $60 / 40 \mathrm{~T}+\mathrm{G}$ & 462 & 50 & 438 & 50 & 469 \\
\hline $\mathrm{E} 4$ & $80 / 20 \mathrm{~T}+\mathrm{G}$ & $640^{\circ}$ & 50 & 657 & 50 & 684 \\
\hline $\mathrm{T}$ & Terfenol & 930 & 30 & & & \\
\hline
\end{tabular}

Where $\mathrm{E}_{\mathrm{m}}=$ Elastic modulus of matrix

$\lambda_{\mathrm{e}}=$ measured saturation magnetostriction (at $2 \times 10^{6} \mathrm{~A} / \mathrm{m}$ )

$\lambda_{u}, \lambda_{1}=$ saturation magnetostriction predicted by the upper and lower bound models

A comparison of experimental measurements with model calculations based on equations (7-10) and (7-11) is given in Fig.7-2. For a two-component matrix (glass + metal), the value for elastic modulus of the matrix was calculated by avaraging the limiting cases of the uniform strain value (Eqn. 7-3) and the uniform stress value (Eqn. 77). The elastic modulus of the glass was $50 \mathrm{GPa}$ as shown in Table 7-1. Literature values 


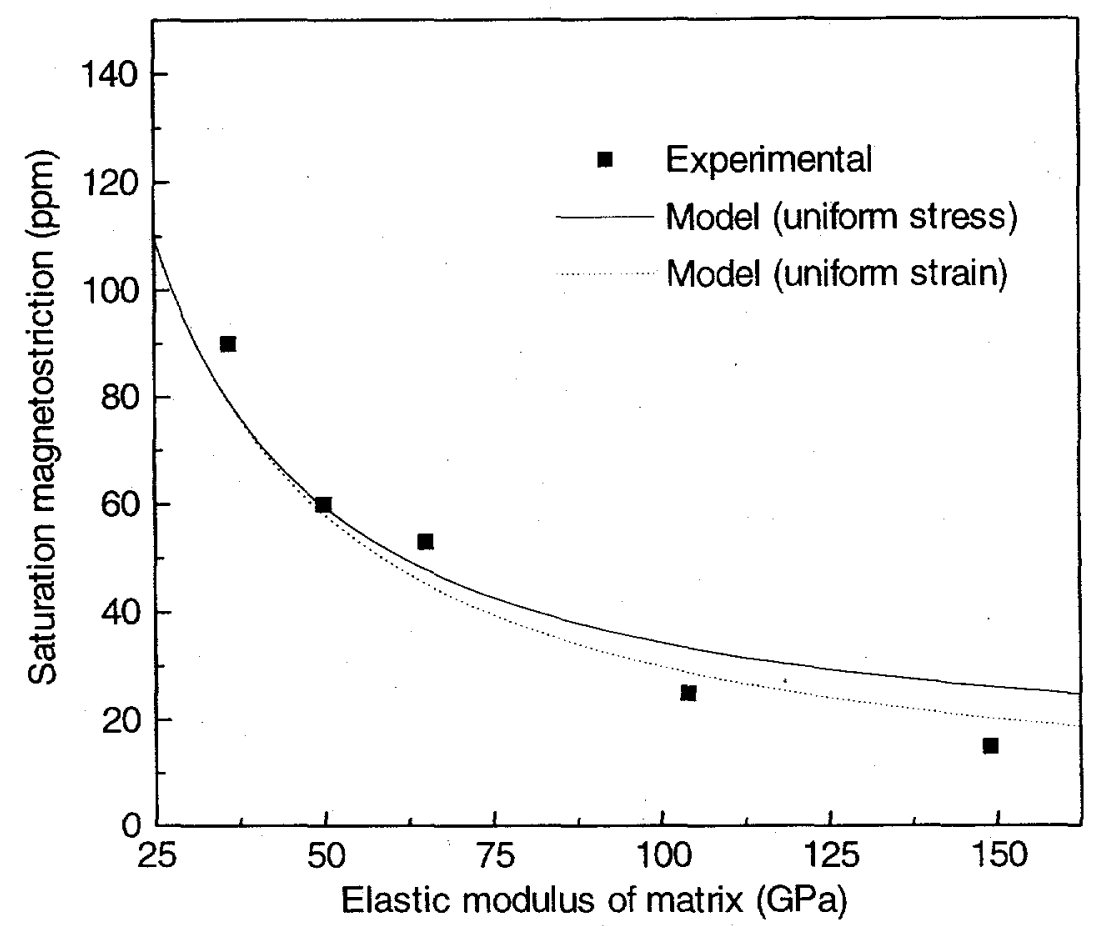

Fig.7-2. Measured and modeled results of composites with fixed volume fraction of magnetostrictive phase and different matrix materials. Symbols are experimental measurement results at $\mathrm{H}=2 \times 10^{6} \mathrm{~A} / \mathrm{m}$ on materials with given values of matrix elastic modulus. The curves are model predictions for the uniform stress approximation and the uniform strain approximation.

were used for the elastic moduli of the various components of the matrix such as $\mathrm{Fe}, \mathrm{Cu}$, $\mathrm{Al}$ and $\mathrm{CeFe}_{2}$ [73]. The model calculations show the predicted magnetostriction as a function of the elastic modulus of the matrix using the known value of $30 \mathrm{GPa}$ for the elastic modulus of the magnetostrictive phase [74]. The measured saturation magnetostriction decreased from $90 \times 10^{-6}$ in a composite with a matrix materials of elastic modulus $36 \mathrm{GPa}$ to a value of $15 \times 10^{-6}$ in a composite with a matrix material of elastic modulus $149 \mathrm{GPa}$. When the elastic moduli of the matrix and magnetostrictive 
phases were similar the predicted limits for the saturation magnetostriction, $\lambda_{\mathrm{se}}$ and $\lambda_{s \sigma}$, were close in value, but the difference between these predicted limits increased with increasing difference between the moduli of the matrix and magnetostrictive phases. These results suggest that the elastic modulus of the matrix material has a significant impact on the saturation magnetostriction of composites, with a lower matrix modulus leading to higher bulk magnetostriction.

Model calculations were performed to determine the magnetostriction as a function of the volume fraction of magnetostrictive phase, again using the known elastic modulus value of $30 \mathrm{GPa}$ for the magnetostrictive phase and a value of $50 \mathrm{GPa}$ for the matrix material. The results are compared with experimental measurements on materials with the same values of matrix elastic moduli as shown in Fig.7-3. In this figure, the solid line represents the modeled results based on the assumption of uniform stress and the dotted line represents the modeled results based on the assumption of uniform strain. It is seen that the experimental results lie very close to the model calculations, and with one exception those values lie between the curves that represent the theoretical upper and lower bounds based on the proposed model.

\subsubsection{Summary}

The model developed in the present work predicts that the magnetostriction of these composites should fall between an upper limit (based on uniform stress) and a lower limit (based on uniform strain). The calculated magnetostrictions obtained on the basis of this model are in good agreement with the experimental results. In both experimental 


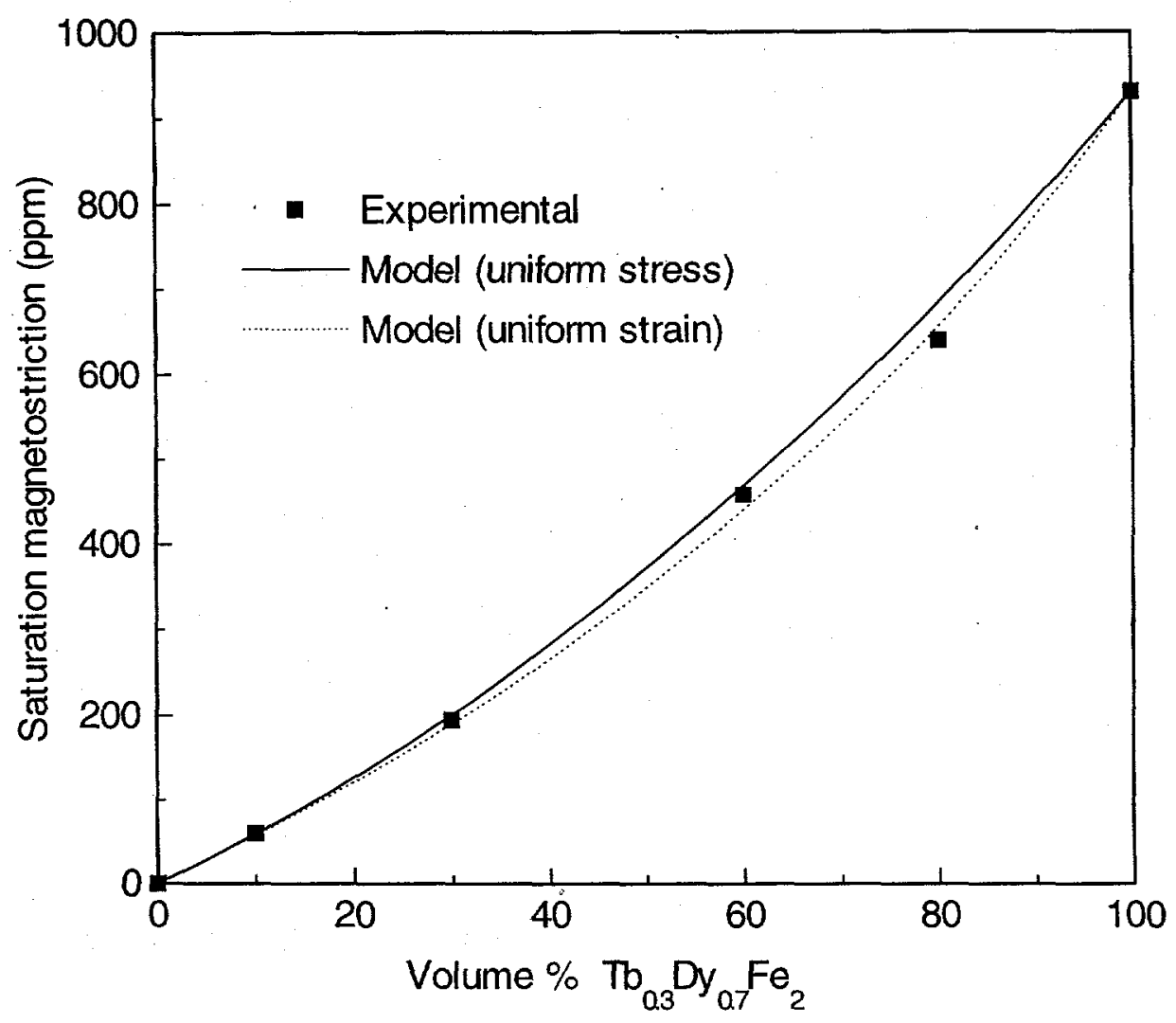

Fig. 7-3 Measured and modeled results for magnetostriction of composites with a matrix phase of known elastic modulus and different volume fractions of magnetostrictive phase. Measurements were made at $\mathrm{H}=2 \times 10^{6} \mathrm{~A} / \mathrm{m}$. Model calculations were made using $\mathrm{E}_{\mathrm{t}}=30 \mathrm{GPa}$, and $\mathrm{E}_{\mathrm{m}}=50 \mathrm{GPa}$.

measurements and modeling the elastic modulus of the matrix seems to play a significant role in determining the bulk magnetostriction of a composite. Specifically the results show that the lower the elastic modulus of the matrix the higher the bulk magnetostriction of the composite. In practical terms the elastic modulus of the matrix can not be too low without adversely affecting the mechanical performance of the material as a whole. In fact matching the elastic moduli of the matrix material and the magnetostrictive phase will in 
many cases be the best way to optimize this situation and obtain the necessary high bulk magnetostriction together with adequate mechanical properties for a sensor material.

\subsection{Magnetomechanical effect model}

Among various modeling approaches $[38,74-78]$, the law of approach to the anhysteretic magnetization was extensively used to model the magnetomechanical effect $[38,45,49]$. Applying the law of approach to the magnetomechanical effect, there are three factors that determine the change of the magnetization of material [38].

- How far the magnetization is above or below the anhysteretic state, i.e. thermodynamic equilibrium state. This may be termed the displacement.

- How sensitive this displacement is to stress (the rate of approach) and

- How the magnetization changes with stress thermodynamically. In other words, how sensitive the anhysteretic curve of the material is to stress.

7.2.1 Effective field due to stress and stress dependent anhysteretic curve

The sensitivity of the anhysteretic to stress is analyzed using an effective field representation of stress. It is usually considered that an applied stress acts in some respects like an applied magnetic field for a magnetostrictive material [77]. The magnetoelastic energy caused by magnetostriction can be expressed by [38]

$$
\mathrm{E}_{\sigma}=-\frac{3}{2} \sigma \lambda\left(\cos ^{2} \theta-v \sin ^{2} \theta\right)
$$


where $\theta$ is the angle between stress $\sigma$ and magnetization, $v$ is Poisson's ratio. Therefore the effective field is obtained by

$$
\mathrm{H}_{\sigma}=-\frac{1}{\mu_{0}} \frac{\partial \mathrm{E}_{\sigma}}{\partial \mathrm{M}}=\frac{3}{2} \frac{\sigma}{\mu_{0}}\left(\frac{\partial \lambda}{\partial \mathrm{M}}\right)_{\sigma}\left(\cos ^{2} \theta-v \sin ^{2} \theta\right)
$$

The bulk magnetostriction $\lambda$ is magnetization dependent and in general is non-linear. An empirical equation can be used to describe the relation between magnetostriction and magnetization based on even order terms of a power series since the magnetostriction must be symmetric about $\mathrm{M}=0$ [38],

$$
\lambda=\sum_{i=1}^{\infty} \gamma_{i} M^{2 i}
$$

where the $\gamma_{i}$ 's are stress-dependent. Considering only the first two terms, then,

$$
\lambda=\gamma_{1} \mathrm{M}^{2}+\gamma_{2} \mathrm{M}^{4}
$$

In a material such as cobalt ferrite or iron, in which magnetostriction peaks at intermediate field, i.e. unsaturated magnetization, the two coefficient $\gamma_{1}$ and $\gamma_{2}$ are opposite in sign and the slope of magnetostriction changes its sign at $M=\left[-\gamma_{1} /\left(2 \gamma_{2}\right)\right]^{1 / 2}$. Since $\gamma_{1}$ and $\gamma_{2}$ are stress-dependent, they can be described by,

$$
\begin{aligned}
& \gamma_{2}=\gamma_{11}+\gamma_{12} \sigma \\
& \gamma_{2}=\gamma_{21}+\gamma_{22} \sigma
\end{aligned}
$$

where $\gamma_{12}, \gamma_{22}$ are negative because the tensile stresses tend to decrease magnetostriction and compressive stresses tend to increase magnetostriction [28, 74, 79]. 
Using these equations, the effective field $\mathbf{H}_{\sigma}$ can be determined at different stress and field levels. With the stress dependent field, the total effective magnetic field is then expressed by

$$
\mathrm{H}_{\mathrm{e}}=\mathrm{H}+\mathrm{H}_{\sigma}+\alpha \mathrm{M}
$$

where $\mathbf{H}$ is the "real" magnetic field, $\mathbf{M}$ is the magnetization and $\alpha$ is Weiss mean field constant representing the coupling between moments. Therefore, the stress-dependence of anhysteretic magnetization curve based on the Langevin function [15], can be expressed by

$$
M_{\text {an }}(H, \sigma)=M_{s}\left[\operatorname{coth}\left(\frac{H+H_{\sigma}+\alpha M}{a}\right)-\frac{a}{H+H_{\sigma}+\alpha M}\right]
$$

where $a=k_{B} T / \mu_{0} m$ and $\mathbf{m}$ is magnetic moment of a typical domain

\subsubsection{Law of approach}

In the law of approach, any change in magnetization is composed of two components: reversible and irreversible components. The reversible component of magnetization is given by [38]

$$
\mathrm{M}_{\mathrm{rev}}=\mathrm{c}\left(\mathrm{M}_{\mathrm{an}}-\mathrm{M}_{\mathrm{irt}}\right)
$$

where $\mathrm{c}$ is the reversibility. The law of approach states that the derivative of the irreversible component is proportional to the displacement of the irreversible component from the anhysteretic magnetization.

$$
\frac{\mathrm{dM}_{\mathrm{irr}}}{\mathrm{dW}}=\frac{1}{\xi}\left(\mathrm{M}_{\mathrm{an}}-\mathrm{M}_{\mathrm{irr}}\right)
$$


where $\xi$ is a coefficient reflecting the rate of approach and $\mathrm{W}$ is the elastic energy generated by applied stress and causing the unpinning of domain walls. Combining equations (7-20) and (7-21) with $M=M_{r e v}+M_{i r}$, the following equation is obtained

$$
\frac{\mathrm{dM}}{\mathrm{dW}}=\frac{1}{\xi}\left(\mathrm{M}_{\mathrm{an}}-\mathrm{M}_{\mathrm{irr}}\right)+\mathrm{c} \frac{\mathrm{d}}{\mathrm{dW}}\left(\mathrm{M}_{\mathrm{an}}-\mathrm{M}_{\mathrm{irr}}\right)
$$

The elastic energy is the product of stress and strain and is given by

$$
\mathrm{dW}=\sigma \mathrm{d} \sigma / \mathrm{E}
$$

where $\mathrm{E}$ is the Young's modulus of material. Then equation (7-22) can be transformed to

$$
\frac{\mathrm{dM}}{\mathrm{d} \sigma}=\frac{\sigma}{\mathrm{E} \xi}\left(\mathrm{M}_{\mathrm{an}}-\mathrm{M}_{\mathrm{irr}}\right)+\mathrm{c} \frac{\mathrm{d}}{\mathrm{d} \sigma}\left(\mathrm{M}_{\mathrm{an}}-\mathrm{M}_{\mathrm{irr}}\right)
$$

or with defining, $\varepsilon^{2}=\mathrm{E} \xi$

$$
\mathrm{dM}=\frac{\sigma}{\varepsilon^{2}}\left(\mathrm{M}_{\mathrm{an}}-\mathrm{M}_{\mathrm{irr}}\right) \mathrm{d} \sigma+\mathrm{c} \cdot \mathrm{d}\left(\mathrm{M}_{\mathrm{an}}-\mathrm{M}_{\mathrm{ir}}\right)
$$

Combining (7-20) and (7-25), the change of magnetization with stress can be expressed by

$$
\mathrm{d} M=\frac{\sigma}{\varepsilon^{2}}(1-\mathrm{c})\left(\mathrm{M}_{\mathrm{an}}-\mathrm{M}_{\mathrm{irr}}\right) \mathrm{d} \sigma+\mathrm{c} \cdot \mathrm{d} \mathrm{M}_{\mathrm{an}}
$$

\subsection{Extension of the model under uniaxial stress to torsional stresses}

If a rod (or a ring) is subjected to torque $\tau$ in the plane normal to its axis, the torsion can be decomposed into biaxial stresses, as shown in Fig.7-4. The stress can be calculated by

$$
\sigma=2 \tau \mathrm{r} / \pi \mathrm{R}^{4}
$$


where $\mathrm{R}$ is the radius of the rod and $\mathrm{r}$ the distance from the axis. In Fig.7- 4, $\mathbf{M}, \mathbf{M}_{\mathrm{a}}$ and $\mathbf{M}_{\mathrm{c}}$ are magnetization, the axial component of magnetization, and circumferential component of magnetization, respectively. $\mathbf{H}_{d}$ is the demagnetizing field associated with $\mathbf{M}_{\mathrm{a}}$ which is dependent on geometry. It has a direction opposite to that of $\mathbf{M}_{\mathrm{a}} \cdot \sigma$ and $-\boldsymbol{\sigma}$ represent the tensile and compressive stresses, respectively.

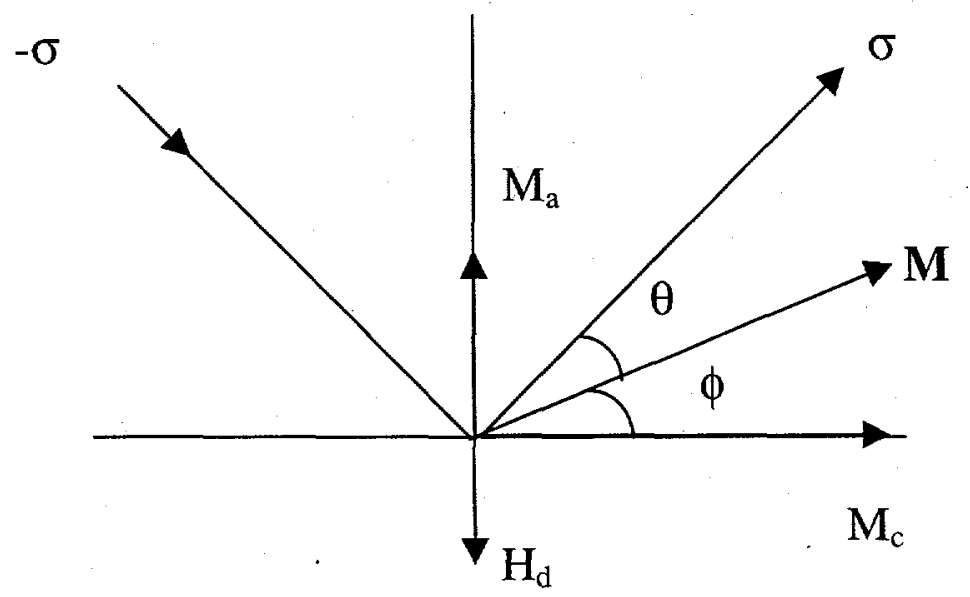

Fig. 7-4 Biaxial stresses in a ring under a torque. The magnetization can be decomposed into two components, circumferential magnetization $\mathbf{M}_{\mathrm{c}}$ and axial magnetization $\mathbf{M}_{\mathbf{a}}$. The demagnetizing field $\mathbf{H}_{\mathrm{d}}$ is associated with $\mathbf{M}_{\mathrm{a}}$ with an opposite direction.

7.3.1 Effective field due to torque

In this case, the magnetoelastic energy can be written as [49]

$$
\mathrm{E}_{\sigma}=-\frac{3}{2} \sigma \lambda(\sigma)\left(\cos ^{2} \theta-v \sin ^{2} \theta\right)+\frac{3}{2} \sigma \lambda(-\sigma)\left(\sin ^{2} \theta-v \cos ^{2} \theta\right)
$$

where $\theta$ is the angle between the magnetization and the tensile stress. Consequently, the effective field to stress can be easily obtained 


$$
\mathrm{H}_{\sigma}=\frac{3 \sigma}{2 \mu_{0}}\left(\left(\frac{\partial \lambda(\sigma)}{\partial \mathrm{M}}\right)\left(\cos ^{2} \theta-v \sin ^{2} \theta\right)-\left(\frac{\partial \lambda(-\sigma)}{\partial \mathrm{M}}\right)\left(\sin ^{2} \theta-v \cos ^{2} \theta\right)\right)
$$

where $\lambda(\sigma), \lambda(-\sigma)$ are the magnetostriction under tension and compressive stress respectively and can be calculated by (7-15) to (7-17).

\subsubsection{Law of approach}

For torsional stress, the total elastic energy is the sum of elastic energy caused by the compressive and tensile stresses, (7-23)

$$
\mathrm{dW}=2 \sigma \mathrm{d} \sigma / \mathrm{E}
$$

The law of approach in this case is expressed by

$$
\mathrm{dM}_{\mathrm{irr}}=2 \frac{\sigma}{\varepsilon^{2}}\left(\mathrm{M}_{\mathrm{an}}-\mathrm{M}_{\mathrm{irr}}\right) \mathrm{d} \sigma
$$

\subsubsection{Rotation of magnetization with respect to torque}

The law of approach above can tell us how the magnetization changes its amplitude. Moreover, the magnetization of the material rotates with respect to torque as well. Garshelis [14] and Sablik [49] derived some formulae to predict the rotation of magnetization under the action of a torque. Both calculations were made under assumptions of thermodynamic equilibrium, i.e. energy minima. The equilibrium angle was calculated by Garshelis [14], considering the demagnetizing energy, anisotropy energy and magnetoelastic energy. 


$$
\phi_{\mathrm{e}}=\frac{1}{2} \tan ^{-1} \frac{3 \lambda \sigma}{\mathrm{K}_{\mathrm{u}}+\mu_{0} \mathrm{~N}_{\mathrm{d}} \mathrm{M}^{2} / 2}
$$

where $\phi$ is the angle of rotation from the original direction needed to minimize the total energy, $K_{u}$ is the uniaxial anisotropy, $N_{d}$ is the demagnetizing factor, $M$ is the remanent magnetization. In this calculation, the magnetization was initially in circumferential direction and the magnetostriction was assumed to be constant. Another concern in this approach is that Garshelis $[10,47,80]$ assumed that the magnitude of magnetization was a constant with applied torque, however this is unlikely.

In the present work, all of these issues are taken account into the calculation. The magnetostriction is considered as dependent on magnetization and stress. The equilibrium deviation of magnetization from its initial direction (either axial or circumferential direction) is expressed by

$$
\phi_{e}=\frac{1}{2} \tan ^{-1} \frac{\frac{3}{2} \sigma \lambda(\sigma)\left(\cos ^{2} \theta-v \sin ^{2} \theta\right)-\frac{3}{2} \sigma \lambda(-\sigma)\left(\sin ^{2} \theta-v \cos ^{2} \theta\right)}{\mathrm{K}_{\mathrm{u}} \pm \mu_{0} \mathrm{~N}_{\mathrm{d}} \mathrm{M}^{2} / 2}
$$

The minus sign in the denominator signifies rotation from axial to circular direction. The plus sign signifies rotation from the circular direction to axial direction. $\mathbf{M}$ is calculated from the law of approach as described in section 7.3.2.

However, the magnetomechanical effect is a kinetic process rather than an equilibrium process, and magnetization is hysteretic and inherently irreversible in nature. Therefore, in the present work, the law of approach of magnetization is extended to the rotation of magnetization. 


$$
\mathrm{d} \phi=\frac{1}{1-\mathrm{c}}\left[\mathrm{cd}\left(\phi_{\mathrm{e}}-\phi\right)+\frac{\sigma}{\varepsilon^{2}}\left(\phi_{\mathrm{e}}-\phi\right) \mathrm{d} \sigma\right]
$$

where $\mathrm{c}$ is the reversibility and $\varepsilon$ reflects the rate of approach.

Combining the changes of both magnitude and direction of magnetization, the change of magnetization as a function of torque could be modeled numerically step by step.

\subsubsection{Modeling procedure}

The magnetization model parameters $\left(M_{s}, a, k, \alpha, c\right)$, can be obtained using the method provided in the literature [81]. The magnetostriction parameters $\left(\gamma_{11} \gamma_{12} \gamma_{21}, \gamma_{22}\right)$ can be determined from the magnetostriction and magnetization curve at different stresses. $\xi$ is a coefficient reflecting the rate of approach and $\mathrm{W}$ is the elastic energy generated by applied stress and causing the unpinning of domain walls. Considering $\varepsilon^{2}=\mathrm{E} \xi$, thus the value of $\varepsilon$ depends on the strength of pinning sites of materials.

The axial magnetic field is the vector sum of the applied field and demagnetizing field. The demagnetizing field is expressed by

$$
\mathrm{H}_{\mathrm{d}}=-\mathrm{N}_{\mathrm{d}} \mathrm{M}_{\mathrm{a}}
$$

where $N_{d}$ is the demagnetizing factor and $\mathbf{M}_{\mathrm{a}}$ is the axial magnetization. The demagnetizing factor is determined by shape of sample. Assuming the axial magnetic field is continuous on the surface of the sample, the surface axial field can be calculated by

$$
\mathrm{H}_{\mathrm{axial}}=\mathrm{H}_{\mathrm{app}}-\mathrm{N}_{\mathrm{d}} \mathrm{M}_{\mathrm{a}} \text {. }
$$

where $\mathbf{H}_{\text {app }}$ is the applied magnetic field. 


\subsubsection{Comparison of experimental and modeled results}

Following the procedure described above, data from both $\mathrm{Ni}$ rods and $\mathrm{Co}$-ferrite composite ring were modeled. The hysteresis loop of an annealed Ni rod is shown in Fig. 7-5. The remanence and the coercivity were measured as $56 \mathrm{kA} / \mathrm{m}$ and $105 \mathrm{~A} / \mathrm{m}$, respectively. Then the model parameters were obtained using the method described in the literature [81], giving. $\mathrm{Ms}=510 \mathrm{kA} / \mathrm{m}, \mathrm{a}=900, \mathrm{k}=2000, \alpha=0.004, \mathrm{c}=0.2 . \quad$ The demagnetizing factor $N_{d}$ was calculated as 0.04 . Since there are few domain wall pinning in the annealed $\mathrm{Ni}, \varepsilon$ was chosen as $4 \times 10^{6}$. The Poisson's ratio of $\mathrm{Ni}$ is 0.31 . The magnetostriction parameters used were: $\gamma_{11}=-9 \times 10^{-17}, \gamma_{12}=-3 \times 10^{-24}, \gamma_{21}=2 \times 10^{-28}, \gamma_{22}=$ $-9 \times 10^{-36}$. The uniaxial anisotropy was chosen as $5000 \mathrm{~J} / \mathrm{m}^{3}$.

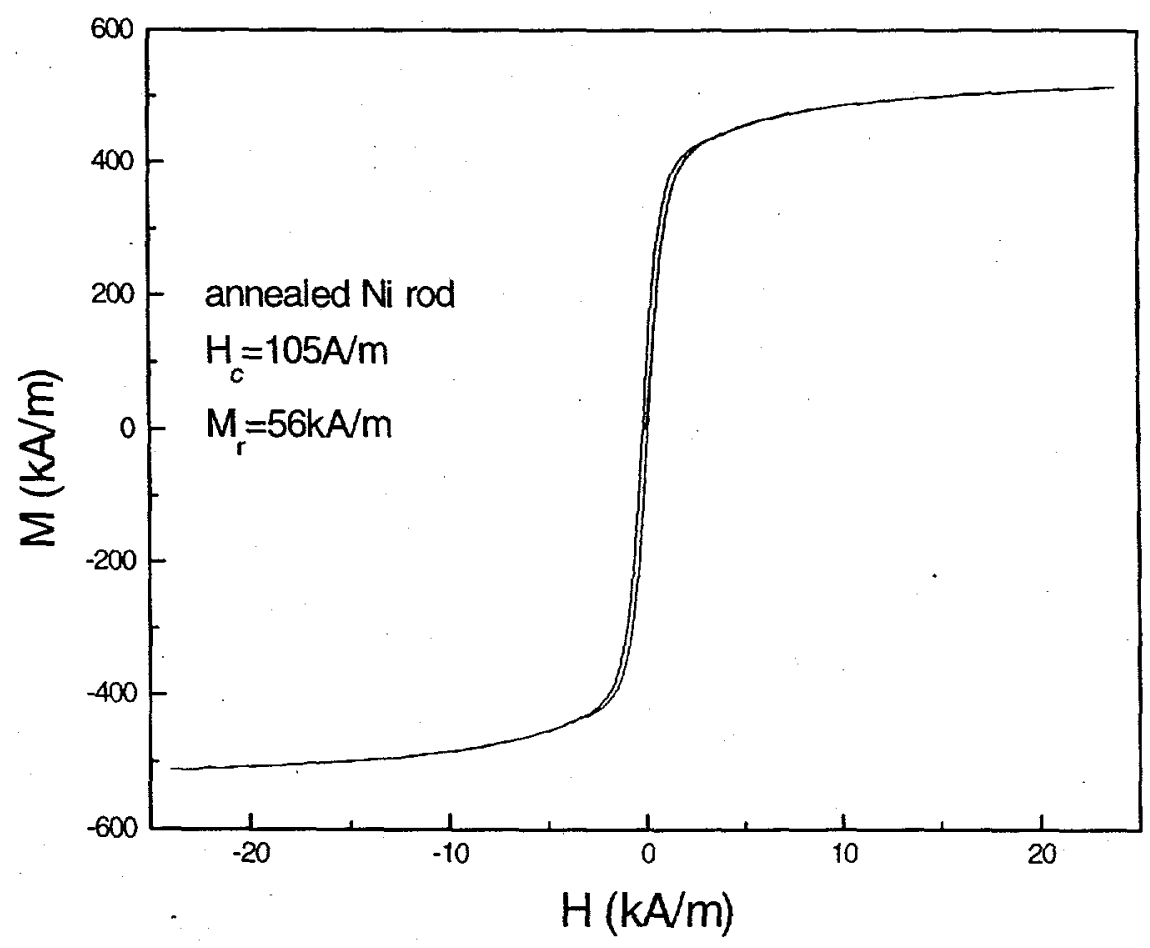

Fig.7-5 Hysteresis loop of an as-annealed Ni rod. 
Using these parameters, the modeled results of $\sigma-\mathrm{H}$ processes of the as-annealed $\mathrm{Ni}$ rod are shown in Fig. 7-6. The axial magnetization under torque of $10 \mathrm{~N} \cdot \mathrm{m}$ was slightly higher than the magnetization without torque. This was due to the increase of the effective magnetic field from the applied torque. This trend agrees with our experimental results and also the results of Sablik's model [24].

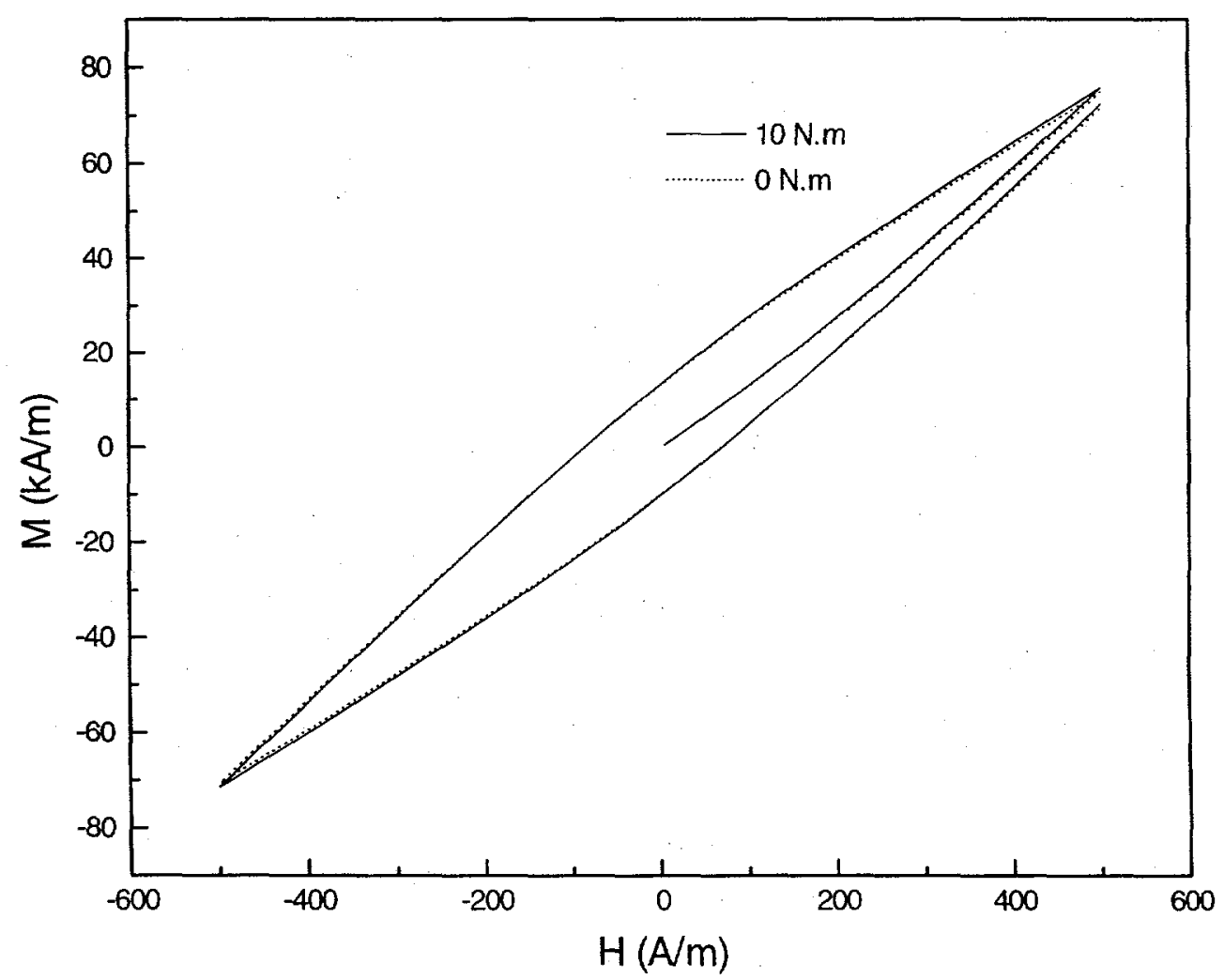

Fig 7-6 Modeled $\sigma-\mathrm{H}$ process of an as-annealed Ni rod with an outer diameter $13 \mathrm{~mm}$. The dotted line represented the hysteresis loop without torque. The solid line represents the hysteresis loop under an applied torque of $10 \mathrm{~N} \bullet \mathrm{m}$. 
The H- $\sigma$ process was modeled for the as-annealed Ni rod as well. After applying a $2.5 \mathrm{kA} / \mathrm{m}$ magnetic field, the change of surface field with torque was modeled. The results are shown in Fig.7-7. The magnetization of the sample increased with the application of torque, and approached its anhysteretic magnetization, which was calculated as $72.4 \mathrm{kA} / \mathrm{m}$ by equation $7-19$ and $7-29$. As the magnetization increased, the demagnetizing field increased so that the surface magnetic field decreased as shown in Fig. 7-7(b). The results agree well with the experimental results in Fig. 6-12.

It was difficult to model the magnetomechanical effect of as-machined $\mathrm{Ni}$ rods entirely from measured data because the applied stresses from torque are just the biased stresses superimposed on the residual stresses of the samples. The residual stresses are obviously not uniform in the materials. In fact, the sum of residual stresses is always zero in a material. Another difficulty in modeling magnetomechanical effect of $\mathrm{Ni}$ rod at remanence from the experiments is that there is not an easy way to estimate the circumferential field although similar behavior of circumferential magnetization can be modeled.

In the modeling of ring shape materials, the magnetization was initialized in the circumferential direction, i.e. circumferential remanent magnetization. Then the magnetization rotated towards tensile or compressive stress axis, which was 45 degrees from its initial direction and then an axial component of magnetic field arose. Using the different algorithm described in 7.3.3, the magnetomechanical effect of Co ferrite composite with a ring shape, i.e. the prototype torque sensor was modeled. 


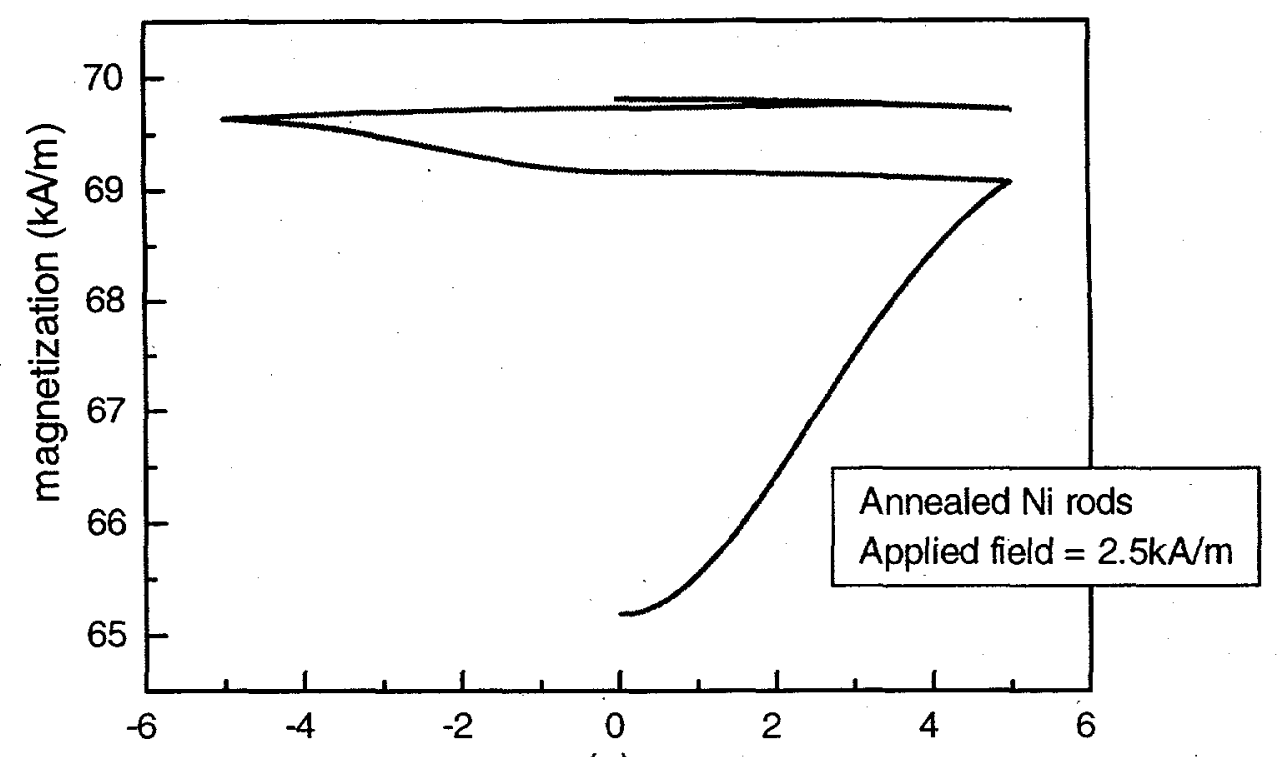

(a)

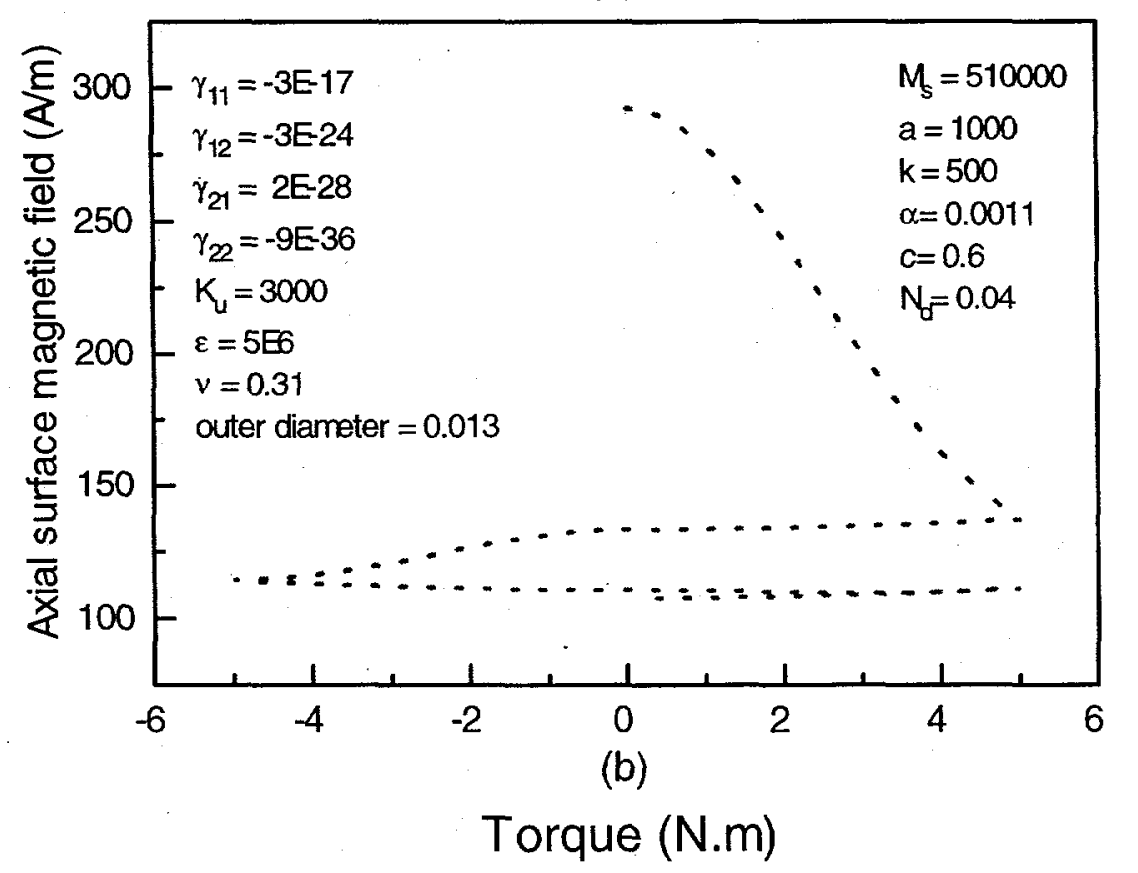

Fig. 7-7 Modeled $\mathrm{H}-\sigma$ process of an as-annealed $\mathrm{Ni}$ rod. The result was measured at an applied field of $2.5 \mathrm{kA} / \mathrm{m}$. (a) the change of magnetization as a function of torque. (b) the change of surface field with applied torque. 
The magnetization curve of the metal-bonded Co ferrite composite (2vol\% $\left.\mathrm{Ag}_{0.97} \mathrm{Ni}_{0.03}+98 \mathrm{vol} \% \mathrm{CoO} \cdot \mathrm{Fe}_{2} \mathrm{O}_{3}\right)$ at room temperature, is shown in Fig. 7-8. The remanence and coercivity are $48 \mathrm{kA} / \mathrm{m}$ and $8 \mathrm{kA} / \mathrm{m}$, respectively. The magnetization model parameters were chosen through the method described above, $\mathrm{Ms}=450 \mathrm{kA} / \mathrm{m}$, a $=600, \mathrm{k}=2000, \alpha=0.0011, \mathrm{c}=0.2$. A demagnetizing factor $\mathrm{N}_{\mathrm{d}}=0.05$, was chosen. The pinning sites of ferrite materials are much stronger than nickel, so $\varepsilon$ was chosen to be $4 \times$ $10^{8}$. The Poisson's ratio of $\mathrm{Ni}$ was chosen as 0.20 . The magnetostriction curve of this material was shown in Fig. 6-6.

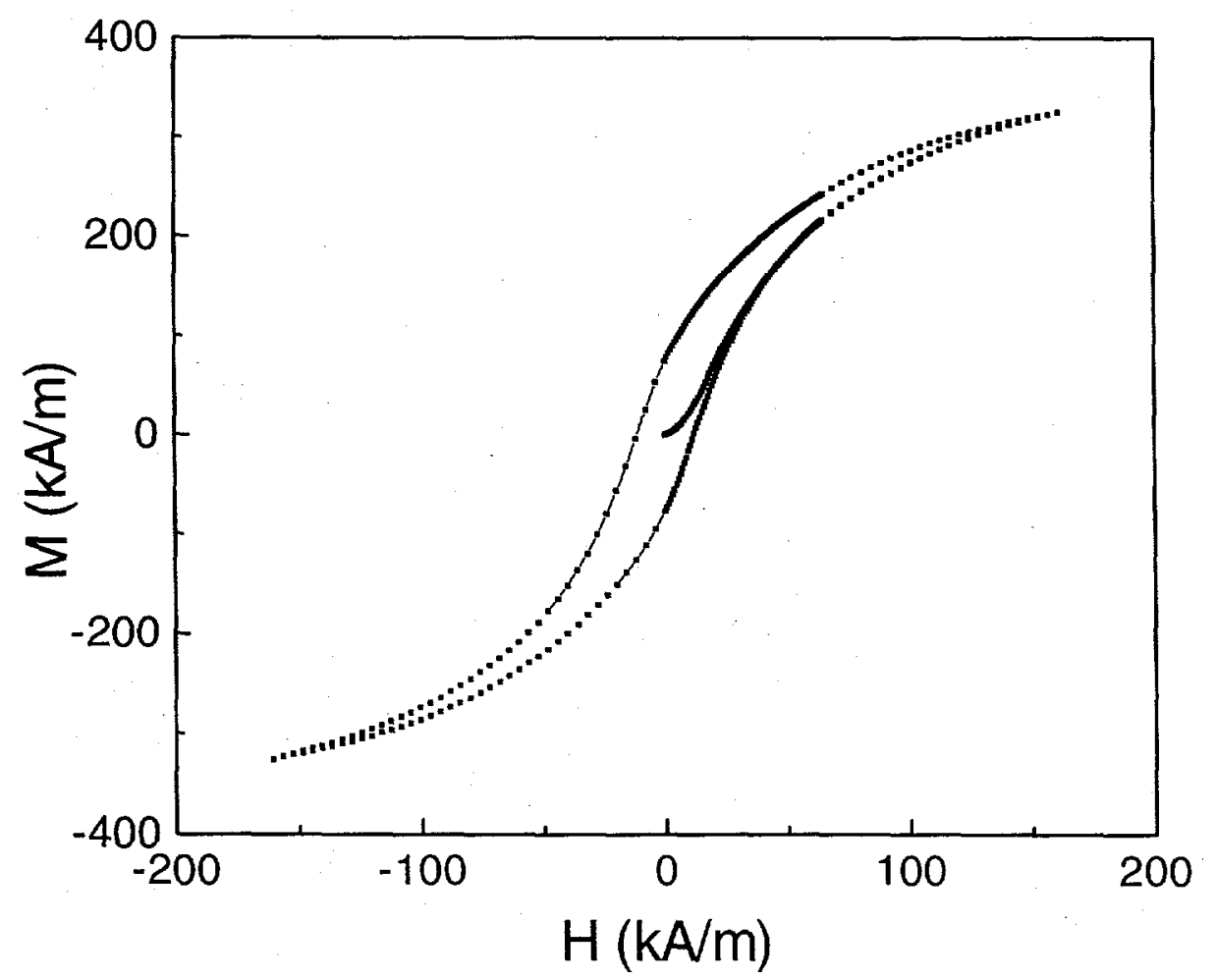

Fig. 7-8 Magnetization curve of the metal-bonded Co ferrite composite

$$
\left(2 / 98 \mathrm{vol} \% \mathrm{Ag}_{0.97} \mathrm{Ni}_{0.03}+\mathrm{CoO} \cdot \mathrm{Fe}_{2} \mathrm{O}_{3}\right)
$$


Using the method described in 7.2.1, the magnetostriction parameters $\left(\gamma_{11} \gamma_{12} \gamma_{21}\right.$, $\gamma_{22}$ ) were approximately chosen $\gamma_{11}=-9 \times 10^{-17}, \gamma_{12}=-3 \times 10^{-24}, \gamma_{21}=2 \times 10^{-28} \gamma_{22}=-9 \times 10^{-36}$. The uniaxial anisotropy used was $10 \mathrm{~kJ} / \mathrm{m}^{3}$. The modeled result showing the change of surface axial field as a function of applied torque is plotted in Fig. 7-9. The model result has a sensitivity of $61 \mathrm{~A} / \mathrm{m}$ per $\mathrm{N} \bullet \mathrm{m}$ and a hysteresis $0.4 \mathrm{~N} \bullet \mathrm{m}$. This compared favorably with the experimental results in which the sensitivity and hysteresis were $65 \mathrm{~A} / \mathrm{m}$ per $\mathrm{N} \cdot \mathrm{m}$ and $0.5 \mathrm{~N} \cdot \mathrm{m}$, respectively. The model therefore gave quantitatively numerical values for the ring sample configurations.

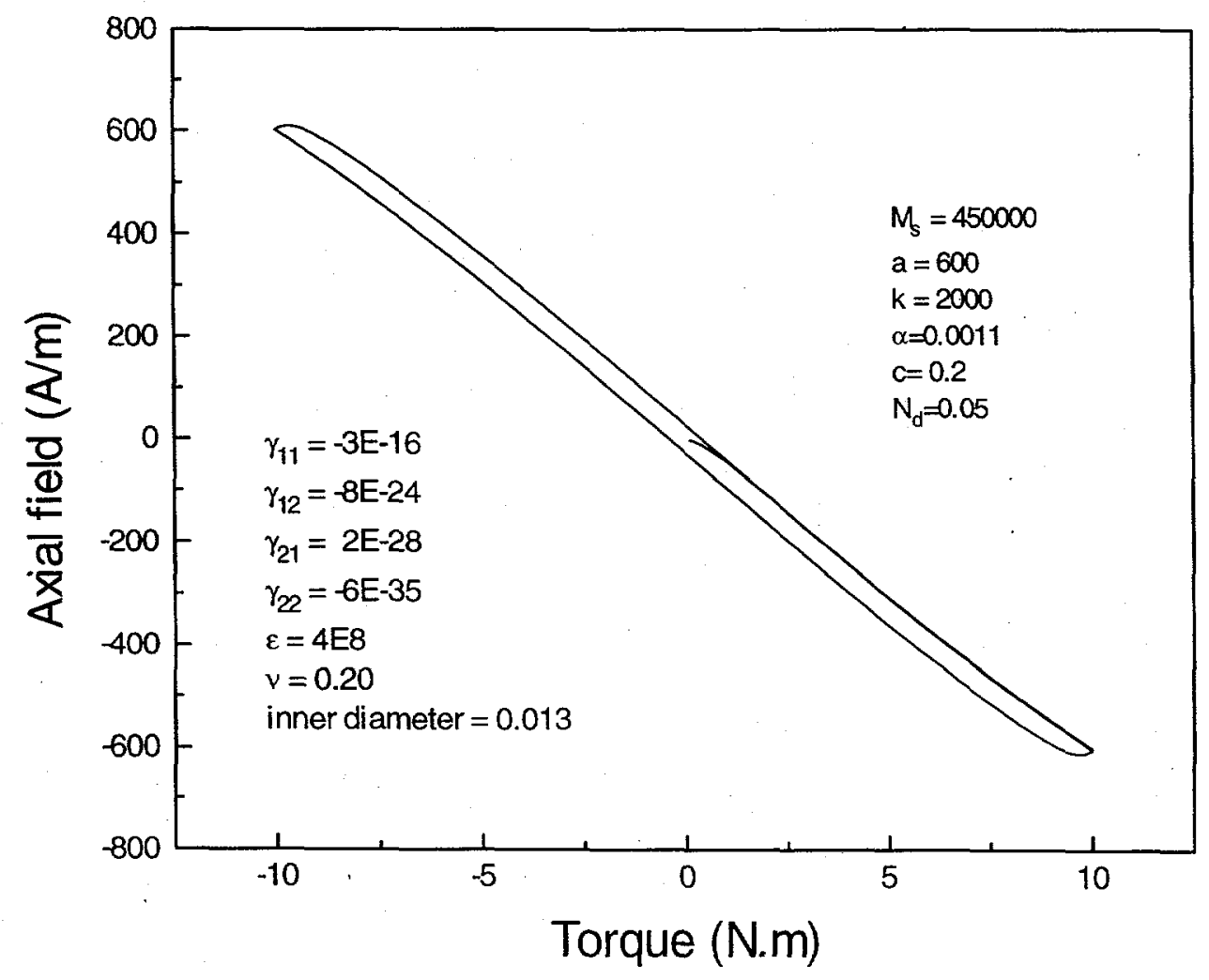

Fig. 7-9 Modeled result of magnetomechanical effect of Co ferrite ring under torque. The sensitivity and hysteresis were $61 \mathrm{~A} / \mathrm{m}$ per $\mathrm{N} \bullet \mathrm{m}$ and $0.4 \mathrm{~N} \bullet \mathrm{m}$, respectively. 


\subsubsection{Summary}

Two critical factors were summarized from the modeling above, $\varepsilon$ and $\mathrm{N}_{\mathrm{d}}$. For soft magnetic materials, (e.g. as-annealed $\mathrm{Ni}$ ), the domain wall pinning is weak, $\varepsilon$ is small, then the magnetization approaches to its anhysteretic state easily and the irreversible components dominate the magnetomechanical processes. Then soft materials are not suitable for the torque sensing applications, especially for the remanent sensing mode used in the present study. For relatively hard magnetic materials, (e.g. Co ferrites), the domain wall pinning is strong and $\varepsilon$ is large and then the remanent magnetization is stable.

The demagnetizing factor has two opposite effects on the performance, specifically on the sensitivity of the prototype sensor. First of all, the change of axial surface magnetic field is proportional to the demagnetizing factor according to equation 7 35. Secondly, the demagnetizing factor impedes the rotation of the magnetization from the circumferential direction, (equation 7-33). It is known, the demagnetizing factor of ring shape is determined by the height/diameter ratio. Therefore, adjusting the height/diameter can optimize the sensitivity of the prototype torque sensor. 


\section{CONCLUSIONS}

The effect of the matrix material on the magnetostriction of composites containing highly magnetostrictive particles was investigated. Experimental results showed that the elastic modulus of the matrix is an important factor determining the bulk magnetostriction of the composite. For a series of composites with the same volume fraction of Terfenol-D particles but different matrix materials, the bulk magnetostriction was found to increase systematically with decreasing elastic modulus of the matrix.

A magnetic torque sensor test bed was developed as part of the present investigation and this was used to make the magnetomechanical measurements under torsional stress. The test bed provided pure applied torque with no associated bending moment, from $-10 \mathrm{~N} \bullet \mathrm{m}$ (counter clockwise, $\mathrm{CCW}$ ) to $+10 \mathrm{~N} \bullet \mathrm{m}$ (clockwise, $\mathrm{CW}$ ) using a computer-controlled servo motor. The control and data acquisition program written in "Labview" can simulate both $\mathrm{H}-\sigma / \tau$ process and $\tau / \sigma-\mathrm{H}$ process. The test bed can measure major loops as well as minor loops. The temperature function of the test bed can vary the system in the temperature range of $-37^{\circ} \mathrm{C}$ to $90^{\circ} \mathrm{C}$.

Magnetomechanical properties of $\mathrm{Fe}$, $\mathrm{Co}$ and $\mathrm{Ni}$ rods were measured in the test bed. The results on Ni rods showed a linear dependence of external circumferential field on torque. The derivative of surface circumferential magnetic field with respect to torque for a Ni rod was $18.9 \mathrm{AN}^{-1} \mathrm{~m}^{-2}$ in the torque range of $\pm 5 \mathrm{~N} \cdot \mathrm{m}$. Cobalt and iron, on the other hand, showed smaller sensitivity to torsional strain with changes of less than $1 \mathrm{AN}^{-1} \mathrm{~m}^{-2}$. The results suggests that a high initial piezomagnetic coefficient (which determines 
sensitivity), in conjunction with a sufficiently high saturation magnetostriction (which determines dynamic range), are the critical factors in selecting materials for magnetic torque sensors.

Co-ferrite and metal-bonded Co-ferrite composites have been fabricated: These have a combination of relatively high saturation magnetostriction in excess of $200 \mathrm{ppm}$ and a high initial $(\partial \lambda \partial \mathrm{H})_{\sigma}$ of $1.3 \times 10^{-9} \mathrm{~A}^{-1} \mathrm{~m}$. This piezomagnetic coefficient is comparable to polycrystalline Terfenol, and much larger than Terfenol-based composites. The magnetomechanical effect in these materials under torsional stress was measured in the magnetic torque sensor test bed. The changes of surface axial magnetic field in response to applied torque were as high as $65 \mathrm{AN}^{-1} \mathrm{~m}^{-2}$, with only a small hysteresis of $+/-0.5 \mathrm{~N} \cdot \mathrm{m}$. These materials therefore seems promising as candidate materials for torque sensors.

The temperature dependence of the magneto-mechanical effect under torque in Co ferrite composite was investigated. The sensitivity changed from $78 \mathrm{AN}^{-1} \mathrm{~m}^{-2}$ at $-37^{\circ} \mathrm{C}$ to $34.2 \mathrm{AN}^{-1} \mathrm{~m}^{-2}$ at $90^{\circ} \mathrm{C}$ while the magnetomechanical hysteresis decreased from $\pm 1.8 \mathrm{~N} \cdot \mathrm{m}$ at $-37^{\circ} \mathrm{C}$ to negligible above $60^{\circ} \mathrm{C}$. The effects of temperature on magnetomechanical effect under torque at remanence can be described in terms of four processes: changing magnetostriction, changing anisotropy, changing spontaneous magnetization and releasing of domain walls from the pinning sites. Metal-bonded Co ferrite composites show capacity for linear magnetomechanical response to torque. In addition, the metal-bonded composites are mechanically robust, corrosion-resistant, and can be brazed. Thus, metalbonded Co-ferrite composites appear to be prime candidates for practical 
magnetomechanical torque sensors, and for other magnetomechanical sensing and actuating applications.

A magnetostriction model has been developed for magnetostrictive composite materials considering the effect of elastic modulus of matrices. The calculated magnetostrictions based on this model are in good agreement with the experimental results. The magnetomechanical effect under torque was modeled with an extension of the existing uniaxial model of the magnetomechanical effect. This model applies similar principles to an earlier model developed for uniaxial stress. However the implementation is more complicated because of the presence of biaxial stress. The modeled result shows similar behavior to the experimental results and gives quantitatively reasonable results. 


\section{APPENDIX}

List of related publications and conference presentations:

[1]. Y.Chen, and D.C. Jiles, "The magnetomechanical effect under torsional stress and the law of approach in a Co ferrite composite", submitted to Intermag'2000, Toronto, Canada, April 9-13, 2000.

[2]. Y. Chen, J. E. Snyder, K. W. Dennis, R. W. McCallum, and D. C. Jiles, "Temperature dependence of the magnetomechanical effect in metal-bonded cobalt ferrite composites under torsional strain", paper DD-10, accepted for 44th Annual Conference on Magnetism and Magnetic Materials, San Jose, November 1999.

[3]. M. J. Sablik, Y. Chen, and D.C. Jiles, "Modified law of approach for the magnetomechanical model". Presented at the Review of Progress in Quantitative Nondestructive Evaluation, Montreal, Canada, July 26-30, 1999.

[4]. Y. Chen, J. E. Snyder, C. R. Schwichtenberg, K. W. Dennis, R. W. McCallum, and D. C. Jiles, "Metal-bonded Co-ferrite composites for magnetostrictive torque sensor applications", IEEE Trans. Magn. 35, 3652 (1999).

[5]. J. E. Snyder, K.W. Dennis, Y. Chen, R.W. McCallum, D.C.Jiles, "A new magnetoelastic torque sensor material for advanced automotive steering systems", invited paper, 18th Annual Conference on Properties and Applications of Magnetic Materials, Chicago, IL, April, 1999.

[6]. J. E. Snyder, K. W. Dennis, Y. Chen, R. W. McCallum, and D. C. Jiles, "Low coercivity magnetostrictive material with giant piezomagnetic d33", American Physics Society, Centennial Meeting, Atlanta, Georgia, March, 1999.

[7]. Y. Chen, J. E. Snyder, C. R. Schwichtenberg, K. W. Dennis, D. K. Falzgraf, R. W. McCallum, and D. C. Jiles, "Effect of the elastic modulus of the matrix on magnetostrictive strain in composites", Appl. Phys. Lett., 74,1159 (1999).

[8]. Y. Chen, J. E. Snyder, C. R. Schwichtenberg, K. W. Dennis, R. W. McCallum, and D. C. Jiles, "Measurement of magnetomechanical effects under torsional strain in iron, cobalt and nickel", paper HF-09, 43rd Annual Conference on Magnetism and Magnetic Materials, Miami, November 1998.

[9]. J. E. Snyder, Y. Chen, D.C. Jiles, R.W. McCallum, K.W. Dennis, C.R. Schwichtenberg, and D.K. Falzgraf, "Composite magnetostrictive materials for advanced automotive sensors", invited paper, 17th Annual Conference on Properties and Applications of Magnetic Materials, Chicago, IL, May, 1998.

[10]. Y. Chen, J. E. Snyder, C. R. Schwichtenberg, K. W. Dennis, D.K. Falzgraf, R. W. McCallum, and D. C. Jiles, "Effect of matrix on magnetostriction of Terfenol based Composites", U16 3, American Physical Society, March Meeting, Los Angeles, March 1998. 


\title{
Effect of the elastic modulus of the matrix on magnetostrictive strain in composites
}

\author{
Y. Chen, J. E. Snyder, C. R. Schwichtenberg, K. W. Dennis, D. K. Falzgraf, \\ R. W. McCallum, and D. C. Jiles \\ Ames Laboratory, lowa State University, Ames, lowa 50011
}

(Received 26 May 1998; accepted for publication 11 December 1998)

\begin{abstract}
The effect of the matrix material on the magnetostriction of composites containing highly magnetostrictive particles has been studied. Experimental results showed that the elastic modulus of the matrix is an important factor determining the bulk magnetostriction of the composite. For a series of composites with the same volume fraction of magnetostrictive particles but different matrix materials, the bulk magnetostriction was found to increase systematically with decreasing elastic modulus of the matrix. A model theory for the magnetostriction of such composites has been developed, based on two limiting assumptions: uniform strain or uniform stress inside the composite. The theory was then used to predict the magnetostriction of the entire material from the volume fractions of the components, their elastic moduli and magnetostrictions. These predictions were in agreement with the experimental results. It is concluded that to obtain a high magnetostriction and adequate mechanical properties of a composite, the elastic moduli of the magnetostrictive phase and the matrix should be as close as possible in value. 1999 American Institute of Physics. [S0003-6951(99)03106-X]
\end{abstract}

There has recently been interest in the development of magnetomechanical torque sensors ${ }^{1-3}$ because of the sensitivity of magnetic properties to torsional stress. Clark $^{4}$ and Greenough ${ }^{5}$ have discussed rare-earth-iron compounds, however the high cost and brittleness of these materials restrict applications. Composites of highly magnetostrictive particles in a different matrix material, as investigated by Clark and Belson ${ }^{6}$ and by Sandlund $e t a L$, ' ' have the potential for maintaining adequate magnetostriction while meeting mechanical performance specifications. Herbst et al. have also studied $\mathrm{SmFe} / \mathrm{Fe}$ and $\mathrm{SmFe}_{2} / \mathrm{Al}$ composites and described a model to predict the magnetostriction. ${ }^{8,9}$ Recently Nan has also proposed a mathematical model. ${ }^{10}$

In preliminary work magnetostrictive composites were fabricated consisting of highly magnetostrictive particles in a low magnetostriction, high permeability magnetic matrix with the objective of using the high permeability matrix to enhance the magnetic flux and hence the magnetostriction in the particles. The magnetostriction of these materials was measured at various field strengths and this produced unexpected low values.

An experimental study on magnetostriction of composites was undertaken using various matrix materials with different elastic moduli. Results indicated that the elastic modulus of the matrix has a significant effect on magnetostriction. The differences in builk magnetostriction were investigated and, as a result of this, a model theory was developed and verified. This model theory was then used to predict the bulk magnetostriction of such composites.

The composites were prepared by blending powders in an argon atmosphere. The particulate magnetostrictive material used in this study was $\mathrm{Tb}_{0.3} \mathrm{Dy}_{0.7} \mathrm{Fe}_{2}$ while the matrix materials consisted of metal and glass, the metals being $\mathrm{Fe}$, $\mathrm{Cu}, \mathrm{Al}$, or $\mathrm{CeFe}_{2}$. Blended powder was poured into a $6 \mathrm{~mm}$ diàmeter die, pressed at $1-3 \mathrm{kN}$ under an inert argon atmosphere and heated at $10^{\circ} \mathrm{C} / \mathrm{min}$ to $300-600^{\circ} \mathrm{C}$ depending on the matrix material. The load was removed and the material cooled to ambient temperature while still under argon atmosphere. Magnetostriction was measured using strain gauges. The magnetic field was applied parallel to the cylindrical axis of the specimens and the strain was measured along the same direction.

The magnetostriction model was based on the limiting assumptions of uniform stress or uniform strain in an inhomogeneous material. Beginning with the elastic properties, the model predicts that the elastic modulus should lie between two bounds based on these limiting assumptions." The equations for the elastic modulus in the two cases are given below. The magnetostriction of the entire material was calculated in both instances, assuming that the stress in the whole material is generated only by the magnetostriction in the particles, scaled in proportion to the volume fraction of the particulate phase.

Assuming uniform strain throughout a composite material, the total stress in the linear regime over which Hooke's law applies, can be found from

$$
\epsilon E_{c}=\epsilon E_{t} V_{t}+\epsilon E_{m} V_{m},
$$

where $\epsilon=$ strain, $V=$ volume fraction, and $E=$ elastic modulus. The subscript $c$ indicates the whole composite, $m$ indicates the matrix, $t$ indicates the particulate magnetostrictive phase. Equating the strain throughout, the elastic modulus of the composite is given by

$$
E_{c}=E_{l} V_{1}+E_{m} V_{m} \text {. }
$$

Assuming that the stress throughout the material is uniform an alternative expression for the elastic modulus of a composite material is obtained. Again in the linear Hooke's law regime, uniform stress implies the product $E \epsilon$ must be the same for each component, therefore

$$
\sigma=E_{l} \epsilon_{t}=E_{m} \epsilon_{m} .
$$

where $\sigma=$ stress. The strain throughout the entire material is given by 
TABLE I. Measured and modeled saturation magnetostrictions of composites. Matrix materials were: $A\left(\mathrm{Fe}+\right.$ glass), $B\left(\mathrm{Cu}+\right.$ glass) $, C(\mathrm{Al}+$ glass $), D\left(\mathrm{CeFe}_{2}+\right.$ glass $), E$ (glass).

\begin{tabular}{|c|c|c|c|c|c|}
\hline \multirow[b]{2}{*}{ Sample } & \multirow[b]{2}{*}{$\begin{array}{c}\text { Vol. } \% \\
\text { components }\end{array}$} & \multirow{2}{*}{$\begin{array}{c}\text { Elastic modulus } \\
\text { of matrix } \\
E_{m}(\mathrm{GPa})\end{array}$} & \multirow{2}{*}{$\begin{array}{c}\text { Measured saturation } \\
\text { magnetostriction } \\
\lambda_{e}\left(10^{-6}\right)\end{array}$} & \multicolumn{2}{|c|}{ Predicted magnetostriction } \\
\hline & & & & $\begin{array}{l}\text { Uniform strain } \\
\lambda_{s e}\left(10^{-6}\right)\end{array}$ & $\begin{array}{c}\text { Uniform stress } \\
\lambda_{s,}\left(10^{-6}\right)\end{array}$ \\
\hline$A$ & $1090 T+$ matrix $A$ & 149 & 15 & 20 & 26 \\
\hline$B$ & $10190 T+$ matrix $B$ & 104 & 25 & 28 & 33 \\
\hline$C$ & $10 / 90 T+$ matrix $C$ & 65 & 55 & 46 & 48 \\
\hline$D$ & $10 / 90 T+$ matrix $D$ & 36 & 90 & 79 & 79 \\
\hline$E \mathbf{I}$ & $1090 T+$ matrix $E$ & 50 & 60 & 58 & 60 \\
\hline$E 2$ & $30 / 70 T+$ matrix $E$ & 50 & 200 & 190 & 201 \\
\hline$E 3$ & $60 / 40 T+$ matrix $E$ & 50 & 462 & 441 & 469 \\
\hline$E 4$ & $80 / 20 T+$ matrix $E$ & 50 & 640 & 656 & 684 \\
\hline$T$ & $\quad \mathrm{~Tb}_{0,3} \mathrm{Dy}_{0,7} \mathrm{Fe}_{2}$ & 30 & 930 & & \\
\hline
\end{tabular}

$$
\epsilon_{c}=\epsilon_{s} V_{1}+\epsilon_{m} V_{m} .
$$

Substituting Eq. (3) into (4) gives

$$
\epsilon_{c}=\frac{\sigma}{E_{c}}=\sigma \frac{V_{t}}{E_{t}}+\sigma \frac{V_{m}}{E_{m}}
$$

and the elastic modulus is

$$
E_{\mathrm{c}}=\frac{1}{\frac{V_{t}}{E_{t}}+\frac{V_{m}}{E_{m}}} .
$$

In the magnetostriction model it is assumed that the stress generated in the composite is caused entirely by the magnetostrictive phase and that the overall stress in the composite when it is magnetically saturated is the product of the volume fraction of magnetostrictive phase and the stress exerted by it. Under these conditions the following equation is obtained

$$
E_{c} \lambda_{s}=E_{t} \lambda_{s t} V_{t},
$$

where $\lambda_{s}$ is the saturation magnetostriction of the entire material and $\lambda_{s t}$ is the saturation magnetostriction of the magnetostrictive phase alone. Substituting the expressions for the elastic modulus from Eqs. (2) and (6) gives limiting values of the saturation magnetostriction. For uniform strain a lower limit of magnetostriction is obtained, while for uniform stress an upper limit is obtained

$$
\begin{aligned}
& \lambda_{s \epsilon}=\frac{E_{t} \lambda_{s t} V_{t}}{E_{t} V_{t}+E_{m} V_{m}}, \\
& \lambda_{s \sigma}=E_{t} \lambda_{s t} V_{t}\left(\frac{V_{t}}{E_{t}}+\frac{V_{m}}{E_{m}}\right) .
\end{aligned}
$$

The saturation magnetostriction of a composite material should fall between the values of $\lambda_{s \epsilon}$ and $\lambda_{s \sigma}$ given by these equations.

Magnetostriction curves of two series of samples were measured in order to test these predictions. The first was a series of specimens with a fixed volume fraction of the same magnetostrictive phase in matrix materials with different elastic moduli. The second was a series of specimens with different volume fractions of the magnetostrictive phase in the same matrix material. This allowed the effects of the elastic modulus of the matrix and volume fraction of the magnetostrictive phase to be studied independently. The saturation magnetostriction and elastic moduli of both series of specimens are given in Table I. Magnetostriction curves of the first series of composite materials are shown in Fig. 1. These results show that the magnetostriction of the material increased with decreasing elastic modulus of the matrix. Results on the second series are shown in Fig. 2. These indicate that the saturation magnetostriction of the material increased with increasing volume fraction of the magnetostrictive phase.

A comparison of experimental measurements with model calculations based on Eqs. (8) and (9) is given in Fig. 3 . The model calculations show the predicted magnetostrictions, as a function of the elastic modulus of the matrix, are in good agreement with measurements. For a two-component matrix (glass +metal), the value for elastic modulus of the matrix was calculated by averaging the limiting cases of the uniform strain value [Eq. (2)] and the uniform stress value [Eq. (6)]. The elastic modulus of the magnetostrictive phase was $30 \mathrm{GPa}$ and that of the glass was $50 \mathrm{GPa}$ as shown in Table I. Literature values were used for the elastic moduli of $\mathrm{Fe}, \mathrm{Cu}, \mathrm{Al}$, and $\mathrm{CeFe}_{2}$. The measured saturation magnetostriction decreased from $90 \times 10^{-6}$ in a matrix with elastic modulus of $36 \mathrm{GPa}$ to a value of $15 \times 10^{-6}$ in a matrix with elastic modulus of $149 \mathrm{GPa}$. When the elastic moduli of the matrix and magnetostrictive phases were similar the pre-

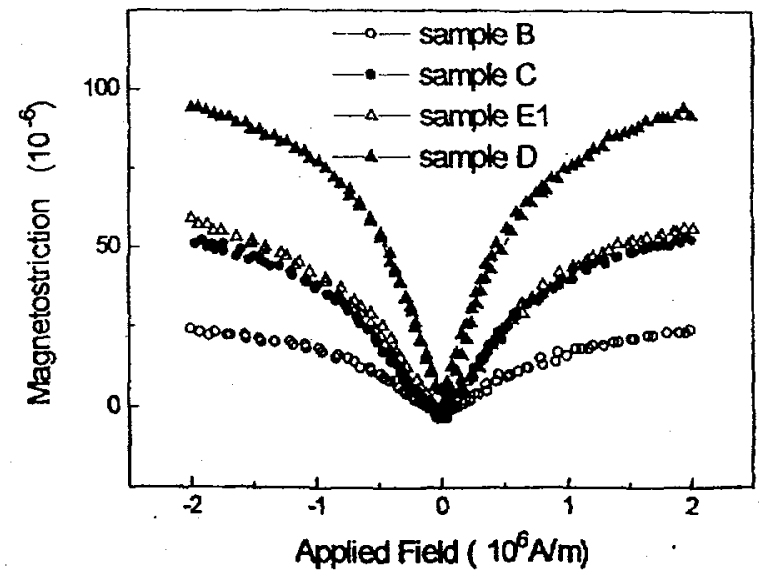

FIG. 1. Magnetostriction curves of several composites with equal volume fractions of magnetostrictive phase particles in different matrix materials. The elastic moduli of the different matrix materials are given in Table $I$. 


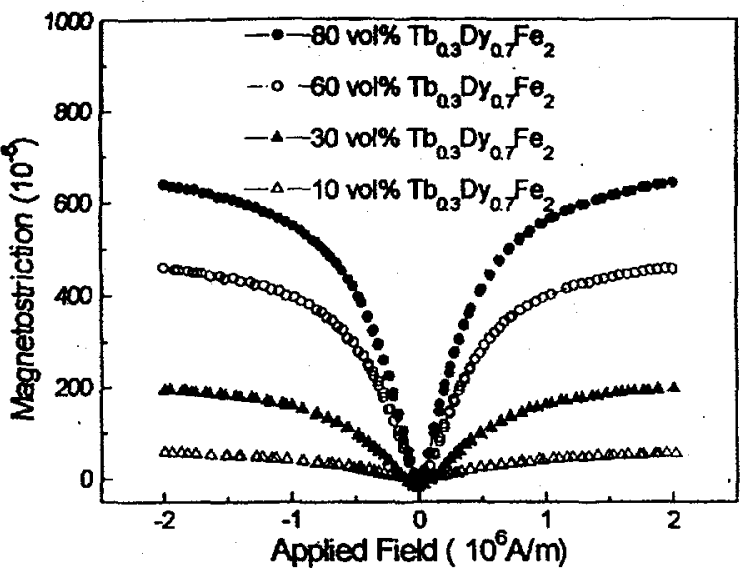

FIG. 2. Magnetostriction curves of several composites with different volume fractions of magnetostrictive phase properties in the same matrix material.

dicted limits for the saturation magnetostriction, $\lambda_{s \epsilon}$ and $\lambda_{s \sigma}$, were close in value, but the difference between these predicted limits increased with increasing difference between the moduli of the matrix and magnetostrictive phases. These results suggest that the elastic modulus of the matrix material has a significant impact on the saturation magnetostriction of composites, with a lower matrix modulus leading to higher bulk magnetostriction.

Model calculations were performed to obtain the magnetostriction as a function of the volume fraction of magnetostrictive phase using the same elastic modulus values of $\mathbf{3 0}$ GPa for the magnetostrictive phase and $50 \mathrm{GPa}$ for the matrix. The results are compared with experimental measurements on a series of materials with the same values of matrix elastic moduli as shown in Fig. 4. In this figure, the solid line represents the modeled results based on the assumption of uniform stress and the dotted line represents the modeled results based on the assumption of uniform strain.

The model developed in the present work predicts that the magnetostriction of these composites should fall between an upper limit (based on uniform stress) and a lower limit

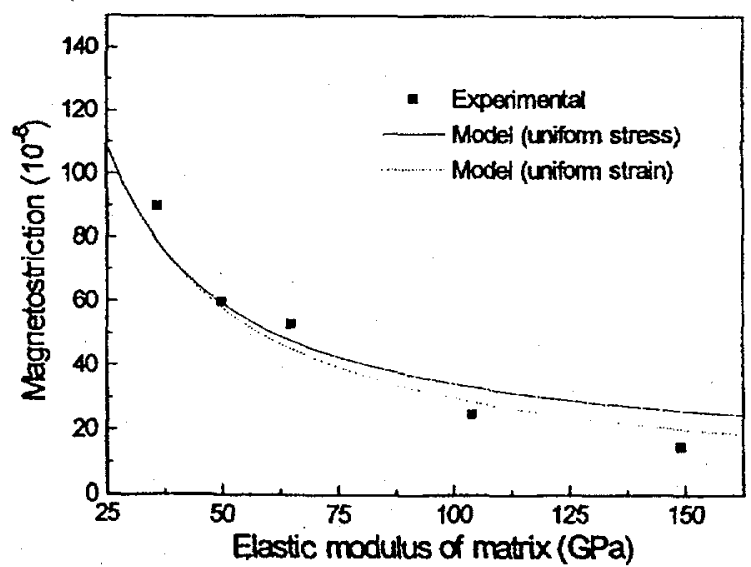

FIG. 3. Measured and modeled results of composites with fixed volume fraction of magnetostrictive phase and different matrix materials. Symbols are experimental measurement results at $H=2 \times 10^{6} \mathrm{~A} / \mathrm{m}$ on materials with given values of matrix elastic modulus. The curves are model predictions for the uniform stress approximation and the uniform strain approximation.

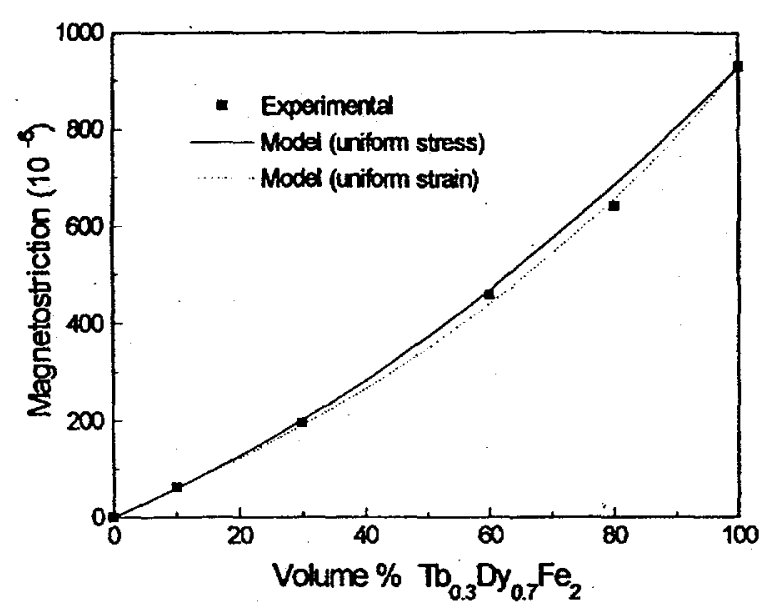

FIG. 4. Measured and modeled results for magnetostriction of composites with a matrix phase of known elastic modulus and different volume fractions of magnetostrictive phase. Measurements were made at $H=2$ $\times 10^{6} \mathrm{~A} / \mathrm{m}$. Model calculations were made using $E_{\mathrm{r}}=30 \mathrm{GPa}$, and $E_{m}$ $=50 \mathrm{GPa}$.

(based on uniform strain). The calculated magnetostrictions obtained on the basis of this model are in good agreement with the experimental results. In both experimental measurements and modeling the elastic modulus of the matrix seems to play a significant role in determining the bulk yagnetostriction of a composite. Specifically the results show that the lower the elastic modulus of the matrix the higher the bulk magnetostriction of the composite. In practical terms the elastic modulus of the matrix cannot be too low without adversely affecting the mechanical performance of the material as a whole. In fact matching the elastic moduli of the matrix material and the magnetostrictive phase is probably the way to optimize this situation and obtain the necessary high bulk magnetostriction together with adequate mechanical properties for a magnetostrictive composite.

Ames Laboratory is operated by the U.S. Department of Energy by Iowa State University - under Contract No. W-7405-ENG-82. This work was supported by the Office of Energy Research, Office of Computational and Technology Research, Advanced Energy Projects Division. The authors would like to thank the referee for calling attention to the work of C.-W. Nan, which was published after this letter was originally submitted.

'W. J. Fleming, Society of Automotive Engineers, 890482 and 890483 , 1989 (unpublished).

${ }^{2}$ J. F. Herbst and F. E. Pinkerton, J. Magn. Magn. Mater. 176, 183 (1997).

3. I. J. Garshelis, IEEE Trans. Magn. 28, 2202 (1992).

'A. E. Clark, Ferromagnetic Materials, Vol. 1, edited by E. P. Wohlfarth (North-Holland, Amsterdam, 1980), pp. 531-589.

${ }^{5}$ R. D. Greenough, Intermetallic Compounds: Vol. 2, Practice, edited by J. H. Westbrook and R. L. Fieischer (Wiley, New York, 1994), pp. 389-405.

${ }^{6}$ A. E. Clark and H. S. Belson, US Patent No. 4,378,258 (March 1983).

'L. Sandlund, M. Fahlander, T. Cedell, A. E. Clark. J. B. Restorf, and M. Wun-Fogle, J, Appl. Phys. 75, 5656 (1994).

${ }^{8}$ F. E. Pinkerton, T. W. Capehart, J. F. Herbst, E. G. Brewer, and C. B. Murphy. Appl. Phys. Lett. 70, 2601 (1997).

${ }^{9} \mathrm{~J}$. F. Herbst, T. W. Capehan, and F. E. Pinkerton, Appl. Phys. Lett. 70. 3041 (1997).

${ }^{10}$ C. W. Nan, Appl. Phys. Lett. 72, 2897 (1998).

"W. D. Callister, in Materials Science and Engineering: An Introduction. 4th ed. (Wiley, New York, 1997), p. 513 


\title{
Metal-bonded Co-Ferrite Composites for Magnetostrictive Torque Sensor Applications
}

\author{
Y. Chen, J. E. Snyder, C. R. Schwichtenberg, K. W. Dennis, R. W. McCallum, D. C. Jiles \\ Ames Laboratory, lowa State University, Ames, lowa 50011
}

\begin{abstract}
A new class of magnetomechanical sensor materials, Co-ferrite ( $\mathrm{CoO} \cdot \mathrm{Fe}_{2} \mathrm{O}_{3}$ ) and metal-bonded $(\mathrm{Ag}, \mathrm{Ni}$, Co) Co-ferrite composites, has been investigated. These materials exhibited magnetostriction in excess of $200 \mathrm{ppm}$ and high $d_{33}(\partial \lambda / \partial H)_{\sigma}$ coefficient, $1.3 \times 10^{-9} A^{-1} \mathrm{~m}$, at low applied field $(<100 \mathrm{kA} / \mathrm{m})$. Selected compositions were formed into test samples in the form of rings brazed to stainless steel through-shafts. Changes of surface axial magnetic field in response to applied torque as high as $64 \mathrm{AN}^{-1} \mathrm{~m}^{-2}$ were observed for a demonstration sample of dimensions $25 \mathrm{~mm}$ $O D, 12.5 \mathrm{~mm} \mathrm{ID}$, and $8 \mathrm{~mm}$ high. A hysteresis of $\pm 0.5 \mathrm{~N} \cdot \mathrm{m}$ was observed. These materials appear to be promising candidates for torque sensors, and other magnetostrictive sensor and actuator applications.
\end{abstract}

Index Terms-Ferrite, torque sensor, magnetostriction, magnetomechanical effect.

\section{INTRODUCTION}

The need for non-contact torque sensors has sparked considerable interest in developing new magnetomechanical sensor materials [1-5]. Highly magnetostrictive materials, such as Terfenol $\left(\mathrm{Tb}_{0.3} \mathrm{Dy}_{0.7} \mathrm{Fe}_{2}\right)[6], \mathrm{SmFe}_{2}$ [7] and other rare-earth-iron compounds have been considered as candidates for such applications. These materials have very high saturation magnetostrictions (1000-3000ppm) [6], and might reasonably be expected to have high sensitivity of magnetization to stress and therefore be suitable for magnetomechanical sensor applications. Composites based on rare-earth compounds have been studied by Pinkerton et al [7], and Chen et al [8]. However, the high anisotropy of these materials causes low sensitivity to stress $[4,9]$ and thus restricts possible applications for magnetomechanical sensors. Maraging steel has been considered an alternative sensor material by Garshelis [2], however the low magnetostriction amplitude of maraging steels can be problematic. In the present work, we propose a new class of magnetomechanical sensor materials: Co-ferrite and metal-bonded $\mathrm{Co}$-ferrite composites. The materials are inexpensive and non-corroding, and the metal-bonded $\mathrm{Co}-$ ferrite composites are mechanically robust and can be attached (e.g. by brazing) to metal parts. They show a steep slope of magnetostriction at low applied fields, $(\mathrm{d} \lambda \mathrm{dH})_{\sigma}$, which contributes to a high sensitivity of magnetic induction to stress, hence giving high signal-tobackground noise ratios in sensor applications.

Manuscript received March 5, 1999.

Y.Chen, 515-294-4140, fax 515-294-8727, yhchen@iastate.edu

\section{EXPERIMENTAL DETAILS}

Co ferrite powder was prepared by blending stoichiometric amounts of fine, high purity cobalt oxide and iron oxide powders in a ball mill then firing at $1100^{\circ} \mathrm{C}$ for 72 hours in flowing dry air to form the compound. The Co ferrite compound powder was then milled to $<38 \mu \mathrm{m}$ and blended with chosen ratios of fine metal powders $(\mathrm{Ag}$, $\mathrm{Ni}, \mathrm{Co}$ ) to establish the metal bonded composite starting powder. These powders were cold pressed into shapes and sintered at up to $1450^{\circ} \mathrm{C}$. The resulting samples were typically cylinders of $4-5 \mathrm{~mm}$ in diameter and $10-15 \mathrm{~mm}$ in length. Magnetostriction was measured, using strain gauges, as a function of applied magnetic. field. The magnetic field was applied along the cylindrical axis of the samples and the strain was measured in the same direction, as the field was cycled in order to change the magnetization from positive saturation, through zero to negative saturation, then back through zero to positive saturation again. Coercivity and saturation magnetization were also measured for one of the samples. A specimen of composition 98 vol\% CoO $\cdot \mathrm{Fe}_{2} \mathrm{O}_{3}+2 \cdot \mathrm{vol} \% \mathrm{Ag}_{0.97} \mathrm{Ni}_{0.03}$, and dimensions $2 \times 2 \times 4.5 \mathrm{~mm}$ was measured along its long axis in a Quantum Design MPMS SQUID magnetometer.

Selected compositions were pressed and sintered in the form of rings that were brazed onto stainless steel shafts. The magnetic response to torque was measured using a specially designed computer controlled magnetic torque sensor test bed. This provided applied torque with no associated bending moment from $-10 \mathrm{~N} \cdot \mathrm{m}$ (counter clockwise) to $+10 \mathrm{~N} \cdot \mathrm{m}$ (clockwise), using a computercontrolled servomotor. All samples were magnetized circumferentially to remanence before being subjected to torque. To achieve this, the ferrite rings were rotated in the fringing field of a narrow-gap electromagnet, while the field was decreased from $160 \mathrm{kA} / \mathrm{m}$ to 0 . The changes of external axial magnetic fields, close to the surface at the midpoint along the axis, were measured as a function of applied torque.

\section{RESULTS AND DISCUSSION}

In the present study, $\mathrm{Ag} / \mathrm{Ni}$ or $\mathrm{Ag} / \mathrm{Co}$ was used as the metallic constituent in order to bind the ferrite together. $\mathrm{Ag}$, which has a low oxygen affinity, has the advantage of not reducing the ferrite. However the addition of other elements such as $\mathrm{Ni}$ or $\mathrm{Co}$ is required, in order to cause the metal to wet the ferrite properly. This improves the mechanical properties of the Co-ferrite by producing a stronger bond. 
The magnetostriction of a typical Co-ferrite composite with a composition of $2 / 98 \mathrm{vol} \% \mathrm{Ag}_{0.97} \mathrm{Ni}_{0.03}+\mathrm{CoO} \cdot \mathrm{Fe}_{2} \mathrm{O}_{3}$ is compared with a Terfenol-based composite in Fig.l. $\mathrm{CoO} \cdot \mathrm{Fe}_{2} \mathrm{O}_{3}$ is known to have a cubic crystal structure with magnetic easy axes along the $\langle 100\rangle$ directions. The magnetostriction constants $\lambda_{100}$ and $\lambda_{111}$ have opposite signs, and the easy axis magnetostriction constant $\lambda_{100}$ is approximately 5 times the magnitude of $\lambda_{111}$ [10]. The measured magnetostriction of the $\mathrm{CoO} \cdot \mathrm{Fe}_{2} \mathrm{O}_{3}$ sample peaks at relatively low field (about $300 \mathrm{kA} / \mathrm{m}$ ), then decreases with increasing field, as the magnetization of the particles or grains is rotated away from easy directions. This maximum at low applied field contrasts with that of Terfenol-based composites, which only peak at very high applied fields $\left(2 \times 10^{6} \mathrm{~A} / \mathrm{m}\right.$ or larger). Co-ferrite and its metal-bonded composites also have strain detivatives $\left(\partial \lambda / \partial H_{\sigma}\right.$ of $1.3 \times 10^{-9} \mathrm{~A}^{-1} \mathrm{~m}$, at low applied fields $(<100 \mathrm{kA} / \mathrm{m})$, which are comparable to those of polycrystalline Terfenol [11], and much larger than those of Terfenol-based composites, which had values of typically $2 \times 10^{-10} \mathrm{~A}^{-1} \mathrm{~m}$. The coercivity of the specimen of composition 98 vol\% $\mathrm{CoO} \cdot \mathrm{Fe}_{2} \mathrm{O}_{3}+2$ vol\% $\mathrm{Ag}_{0.97} \mathrm{Ni}_{0.03}$ was measured to be $6.8 \mathrm{kA} / \mathrm{m}$, and its saturation magnetization $78 \mathrm{Am}^{2} / \mathrm{kg}$.

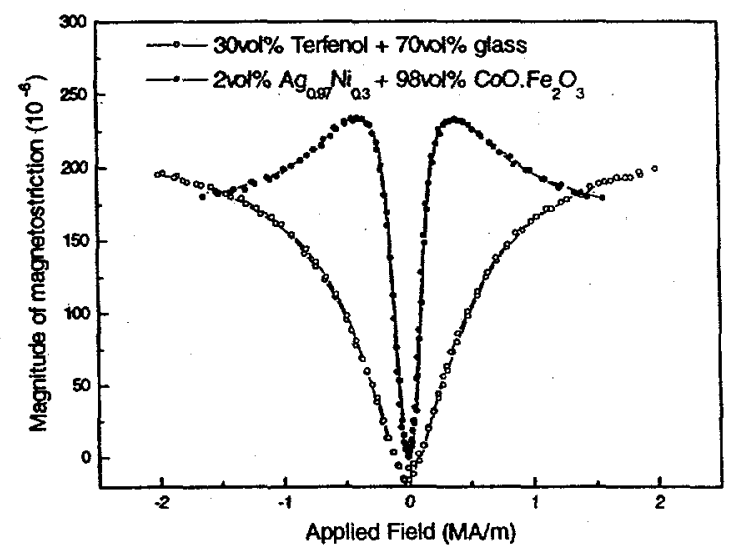

Fig. I Comparison of magnetostrictions of Co-ferrite composite and Terfenol composite

The maximum magnetostrictions of pure sintered $\mathrm{Co}$ ferrite $\left(\mathrm{CoO} \cdot \mathrm{Fe}_{2} \mathrm{O}_{3}\right)$ and some examples of metal-bonded composites are shown in Table I. Although there is very little single crystal Co-ferrite data reported in the literature, a value of $-250 \times 10^{-6}$ is reported for $\lambda_{100}$ for a sample of composition $\mathrm{Co}_{1.1} \mathrm{Fe}_{1.9} \mathrm{O}_{4}$ [10]. While some of the metal-bonded composites have lower peak magnetostriction than pure $\mathrm{CoO} \cdot \mathrm{Fe}_{2} \mathrm{O}_{3}$, they generally offer better mechanical properties and higher slope of magnetostriction $(\partial \lambda / \partial \mathrm{H})_{\sigma}$ at low applied field $(<100$ $\mathrm{kA} / \mathrm{m}$ ), and are therefore more suitable for sensor and actuator applications.

The torque response of a demonstration Co-ferrite ring sample brazed to a stainless steel shaft is shown in Fig.2. The sample had a composition of 98 vol\%
$\mathrm{CoO} \cdot \mathrm{Fe}_{2} \mathrm{O}_{3}+2$ vol\% $\mathrm{Ag}_{0.97} \mathrm{Ni}_{0,03}$, and dimensions $25 \mathrm{~mm}$ $\mathrm{OD}, 12.5 \mathrm{~mm} \mathrm{ID}$, and $8 \mathrm{~mm}$ high. The magnetomechanical sensitivity to applied torque was $64 \mathrm{~A} / \mathrm{m}$ per $\mathrm{N} \bullet \mathrm{m}$ with a hysteresis of $+/-0.5 \mathrm{~N} \bullet \mathrm{m}$.

TABLE I

Magnetostrictions of $\mathrm{CoO} \cdot \mathrm{Fe}_{2} \mathrm{O}_{3}$ and various composites bonded with metals

\begin{tabular}{ll}
\hline Composition & Magnetostriction \\
\hline $100 \% \mathrm{CoO} \cdot \mathrm{Fe}_{2} \mathrm{O}_{3}$ & $-225 \mu$ \\
$2 / 98 \mathrm{vol} \% \mathrm{Ag}_{0.5} \mathrm{Ni}_{0.5}+\mathrm{CoO} \cdot \mathrm{Fe}_{2} \mathrm{O}_{3}$ & $-195 \mu$ \\
$2 / 98 \mathrm{vol} \% \mathrm{Ag}_{0.8} \mathrm{Ni}_{0.2}+\mathrm{CoO} \cdot \mathrm{Fe}_{2} \mathrm{O}_{3}$ & $-210 \mu$ \\
$2 / 98 \mathrm{vol} \% \mathrm{Ag}_{0.97} \mathrm{Ni}_{0.03}+\mathrm{CoO} \cdot \mathrm{Fe}_{2} \mathrm{O}_{3}$ & $-225 \mu$ \\
$2 / 98 \mathrm{vol} \% \mathrm{Ag}_{0.98} \mathrm{Ni}_{0.02}+\mathrm{CoO} \cdot \mathrm{Fe}_{2} \mathrm{O}_{3}$ & $-205 \mu$ \\
\hline
\end{tabular}

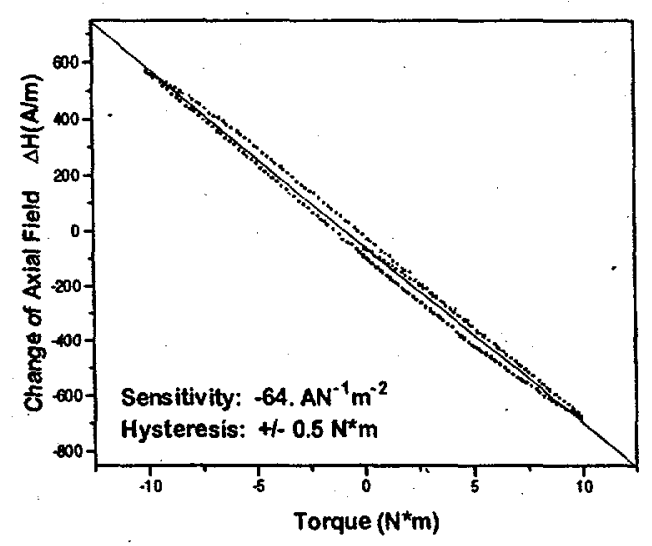

Fig.2. Torque test of metal-bonded $\mathrm{Co}$-ferrite composite ring Composition: $2 / 98 \mathrm{vol} \% \mathrm{Ag}_{0.97} \mathrm{Ni}_{\mathrm{n} .33}+\mathrm{CoO} \cdot \mathrm{Fe}_{2} \mathrm{O}_{3}$

The magnetomechanical effect can be explained in terms of domain processes in magnetostrictive materials. It is known that torsional stresses on a cylinder can be considered to be biaxial stresses, in which the two stress axes are perpendicular to each other and at $45^{\circ}$ to the cylinder axis. The stresses along these two axes are equal in magnitude but opposite in sign [5]. Due to negative magnetostriction, the net magnetization of Co-ferrite composites tends to rotate towards the compressive stress direction [2]. The stresses have two effects on domain wall motion. From a thermodynamic viewpoint, the effect of the stresses can be considered as an effective magnetic field [9], which induces pressure on $90^{\circ}$ domain walls in magnetostrictive materials and leads to domain wall motion. Domain wall motion can be either reversible or irreversible, depending on the strength of the domain wall pinning. If a domain wall is strongly pinned at some points but relatively free to move in-between, application of stress can cause it to bow, much like an elastic membrane. When the stress is removed, it can return to its original position, thus giving reversible behavior. It is the reversible part of the magnetoelastic coupling that determines the sensitivity of the magnetomechanical 
sensor material. However, if this effective field is strong enough to release a domain wall from a pinning site and move it ahead to another location, the domain wall will not return when the stress is removed, thus causing irreversible changes in magnetization, and hysteresis in the magnetomechanical response. Magnetic anisotropy and local variations of magnetic properties contribute to this hysteretic behavior also.

Under reversible conditions the magnetoelastic properties can be discussed in terms of Le Chatelier's principle. For small reversible changes of magnetization, a thermodynamic relation exists, namely

$$
\left(\frac{\partial \lambda}{\partial H}\right)_{\sigma}=\left(\frac{\partial B}{\partial \sigma}\right)_{H}
$$

Thus a relationship between the derivative of magnetostriction with respect to field and the derivative of magnetic induction with respect to stress exists. Note that the sensitivity of magnetization to small applied torsional stress (for example less than $10 \mathrm{~N} \cdot \mathrm{m}$ ) depends principally on the piezomagnetic coefficient $d_{33}=(\partial \lambda / \partial)_{\sigma}$, rather than the saturation magnetostriction $\lambda_{s}$. In view of this, a high piezomagnetic coefficient (which determines sensitivity), in conjunction with a sufficiently high saturation magnetostriction (which determines dynamic range), are the critical factors in selecting materials for magnetic torque sensors. Previously reported experimental results support this conclusion. The measured magnetomechanical sensitivities of rods of $\mathrm{Ni}, \mathrm{Fe}$, and $\mathrm{Co}$ correlated much better with the slopes of magnetostriction $\mathrm{d} \lambda / \mathrm{dH}$ at low field, than with their saturation magnetostriction values [4].

\section{CONCLUSIONS}

Co-ferrite and metal-bonded Co-ferrite composites have been fabricated. These have a combination of relatively high saturation magnetostriction in excess of $200 \mathrm{ppm}$ and low coercivity, which together give a high $(\partial \lambda / \partial)_{\sigma}$ of $1.3 \times 10^{-9} \mathrm{~A}^{-1} \mathrm{~m}$. This is comparable to polycrystalline Terfenol [11], and much larger than Terfenol-based composites, which had derivative values of typically $2 \times 10^{-10} \mathrm{~A}^{-1} \mathrm{~m}$. The magnetomechanical effect under torsional stress was measured in a magnetic torque sensor test bed developed as part of this project. The changes of surface axial magnetic field in response to applied torque were up to $64 \mathrm{AN}^{-1} \mathrm{~m}^{-2}$, with only a small hysteresis of $+/-0.5 \mathrm{~N} . \mathrm{m}$. In addition, the metal-bonded composites are mechanically robust, corrosion-resistant, and can be brazed to shafts. Thus metal-bonded Co-ferrite composites appear to be prime candidates for practical magnetomechanical torque sensors in the range $\pm 10 \mathrm{~N} \cdot \mathrm{m}$, and for other magnetomechanical sensing and actuating applications.

\section{ACKNOWLEDGMENT}

This work was supported by the US Department of Energy, Office of Energy Research, Office of. Computational and Technology Research, Advanced Energy Projects Division. The Ames laboratory is operated by the U.S. Department of Energy by Iowa State University under contract W-7405-ENG-82

\section{REFERENCES}

[1] W.J. Fleming, Society of Automotive Engineers, 890482, 1989.

[2] I. J. Garshelis, IEEE Trans. Magn. 28, 2202 (1992).

[3] I. J. Garshelis, C. R. Conto, J. Appl. Phys., 79, 4756 (1996).

[4] Y. Chen, J. E. Snyder, C. R. Schwichtenberg, K. W. Dennis, R. W. McCallum, and D. C. Jiles, Paper HF-09, $43^{\text {rd }}$ Annual Conference on Magnetism and Magnetic Materials, Nov. 1998.

[5] M.J. Sablik and D.C. Jiles, IEEE Trans. Magn. 35, 498 (1999).

[6] A. E. Clark, in Ferromagnetic Materials, Vol. 1, edited by E. P. Wohlfarth (North-Holland, Amsterdam, 1980), pp531-89.

[7] F. E. Pinkerton, T. W. Capehart, J. F. Herbst, E. G. Brewer; and C. B. Murphy, Appl. Phys. Lett. 70, 2601 (1997).

[8] Y. Chen, J. E. Snyder, C. R. Schwichtenberg, K. W. Dennis, D. K. Falzgraf, R. W. MoCallum, and D. C. Jiles, Appl. Phys. Lett., 74, 1159 (1999).

[9] D. C. Jiles, J. Phys. D: Appl. Phys, 28 (1995) 1537-1546.

[10] R. M. Bozorth, E. F. Tilden, and A. J. Williams, Phys. Rev., 99 $1788,(1955)$.

[11] P. P. Pulvirenti, D. C. Jiles, R. D .Greenough and I. M. Reed, J. Appl. Phys., 79, 6219 (1996). 


\title{
MAGNETO-MECHANICAL EFFECTS UNDER TORSIONAL STRAIN IN IRON, COBALT AND NICKEL
}

Presented in MMM'98 Conference in Miami, Florida, November 1998;

Submitted for publication in J. of Magn. And Magn. Mater, 1999.

Y. Chen, J. E. Snyder, K. W. Dennis, R. W. McCallum, D. C. Jiles

Ames Laboratory, Iowa State University, Ames, Iowa 50011

\begin{abstract}
In the present study, the change of magnetization as a function of applied torque has been investigated for $\mathrm{Fe}, \mathrm{Co}$ and $\mathrm{Ni}$ rods, using torque with no associated bending moment up to $\pm 5 \mathrm{~N} \cdot \mathrm{m}$. The magnetic field near the sample surface was measured using a Hall effect sensor at the remanence point (i.e. zero applied magnetic field). The results showed a linear dependence of this field on torque, which could be advantageous for sensor applications. The derivative of surface circumferential magnetic field with respect to torque for a $\mathrm{Ni}$ rod was $18.9 \mathrm{AN}^{-1} \mathrm{~m}^{-2}$. Cobalt and iron, on the other hand, showed smaller sensitivity to torsional strain with changes of less than $1 \mathrm{AN}^{-1} \mathrm{~m}^{-2}$. The relationship between the magneto-mechanical effect and magnetostriction has also been investigated. It is proposed that the piezomagnetic coefficient (i.e. the slope of the magnetostriction curve) at low magnetic field is a more important figure of merit than saturation magnetostriction in determining sensitivity of magnetization to torque.
\end{abstract}

PACS: $75.80+9,75.60-$ d.

Key words: magnetostriction, magnetomechanical effect, torque, sensor, piezomagnetic coefficient 


\section{Introduction}

The purpose of the present work is to develop better understanding of the magnetomechanical effect under torsional strain and its relationship with magnetostriction in order to develop new materials and sensor geometries for non-contact automotive torque sensor applications.

The magneto-mechanical effect [1], that is the change of magnetization of a magnetic material resulting from the application of stress, has attracted attention recently particularly in the case of torsional stress because of its potential application as a torque sensor [2-8]. Such a torque sensor could make it possible for electromechanical steering systems to replace the costly and fuel inefficient hydraulic power steering systems currently in use. Beth and Meeks [2] first investigated magnetic measurement of torque in a rotating shaft. Non-contacting magnetic torque sensors have been considered to be perhaps the most suitable means of transferring information between computers and mechanical systems such as robotic systems and automobile applications [7].

Magnetic torque sensors can be divided into two categories. The first measures small angles or displacements resulting from torque, using a magnetic circuit [8]. This requires considerable space and is mechanically complicated. The second employs the effect of torque on magnetization in magnetic materials. Most conventional magnetic torque transducers sense the change of permeability or hysteresis parameters with excitation coils under different torques $[2,4,5,7]$. The excitation field makes the detected signal complicated because of the relationship between applied field and magnetization. Garshelis [2] proposed a simple magnetized ring sensor, which did not require external excitation. The surface magnetic field from such a sensor measured by Hall probe was found to be proportional to applied torque. However, the performance of the ring depends on the contact compressive stress with the shaft and the transfer of applied torque from the shaft to the ring. Both of these diminish from a maximum at the ring/shaft interface to zero at the outside surface of the ring. 


\section{Experimental}

A magnetic torque sensor test bed was developed as part of the present investigation and this was used to make the measurements. The test bed provided pure applied torque with no associated bending moment from $-5 \mathrm{~N} \cdot \mathrm{m}$ (counter clockwise, $\mathrm{CCW}$ ) to $+5 \mathrm{~N} \cdot \mathrm{m}$ (clockwise, $\mathrm{CW}$ ) using a computer-controlled servo motor. The precision of the applied torque was $0.05 \mathrm{~N} \cdot \mathrm{m}$. The external magnetic field near the sample surface was measured using a Hall effect sensor with a precision of $0.8 \mathrm{~A} / \mathrm{m}$. The control and data acquisition program was written using "Labview" software.

The dimensions of the $\mathrm{Fe}, \mathrm{Co}$ and $\mathrm{Ni}$ rods are shown in Fig.1. Two square ends were used to fasten the sample to the shaft of the test bed and transfer the torque to the sample. All the rods were magnetized to remanence, i.e., the rods had been saturated axially in an applied field of $53 \mathrm{kA} / \mathrm{m}$ before the magnetomechanical response was measured. The changes of external magnetic fields, including axial field $\left(\mathrm{H}_{\mathrm{a}}\right)$, and circumferential field $\left(\mathrm{H}_{\mathrm{cm}}\right)$, close to the surface at the center of the rods were measured as a function of applied torque. The distance between the Hall sensor and the surface was 0.6mm. For each sample, 10-100 torque cycles were tested. In each cycle, the applied torque was changed from 0 to $5 \mathrm{~N} \cdot \mathrm{m}(\mathrm{CW})$, then reversed to $-5 \mathrm{~N} \cdot \mathrm{m}(\mathrm{CCW})$ and then back to zero. For reference purpose magnetomechanical response was also measured in the demagnetized state.

\section{Results}

In the remanent magnetization state, it was observed that the amplitude of the axial surface field was not a single valued function of the applied torque, but rather depended on the history of torsional strain. The result for the Ni rod is shown in Fig. 2. The squares represent the axial field strength at $-5 \mathrm{~N} \cdot \mathrm{m}$ torque; the stars represent the field strength at 0 torque; and the cycles represent the axial field strength at $5 \mathrm{~N} \cdot \mathrm{m}$. The axial surface field was proportional to the leakage magnetic flux density as well as the axial magnetization of the sample. As the number of torque cycles was increased, the amplitude of the axial field, as well as the magnetization, decreased approximately exponentially and the results 
became more reversible. However, there were still some residual irreversible magnetization processes even after 12 torque cycles as shown in Fig. 3(a). These results also show that there is a small hysteresis in the torque cycle.

Lower sensitivity but also less hysteresis were obtained by using the circumferential field from nickel after several torque cycles. The changes in circumferential field with torque after 12 cycles are shown in Fig. 3 (b). The change of surface circumferential field was $18.9 \mathrm{AN}^{-1} \mathrm{~m}^{-2}$. From to the directions of the axial and circumferential fields, shown in Fig. 3, it was also found that, the direction of total surface field rotated towards the compressive axis under torsional stress.

Cobalt and iron showed smaller sensitivity to torsional strain. The change of circumferential fields of $\mathrm{Fe}$ and Co rods with applied torque were about 0.7 and $0.2 \mathrm{AN}^{-1} \mathrm{~m}^{-2}$.

All of the samples showed no sensitivity to applied torque when measured in the demagnetized state.

\section{Discussion}

All samples were measured in the "as-machined" state, which produces high residual surface stresses. It is these stresses which ensure remanence. (So, applied torsional stresses could be thought of as biased stresses.)

Domain wall motion occurs in response to the torsional stress. Torsional stresses can be considered to be a biaxial stresses in the surface of the material in which the two stress axes are perpendicular to each other, and the stresses along these axes are equal in magnitude but opposite in sign [12]. The stresses have two effects on the domain wall motion $[1,12]$. In view of energy, the first effect of the stresses could be considered as an effective magnetic field, which induces pressure on $90^{\circ}$ domain walls and leads to domain wall motion. It is thermodynamically reversible. Due to negative magnetostriction, the magnetization of nickel would rotate towards to the compressive stress direction [11], while the magnetization in iron, cobalt will rotate towards the tensile axis $[6,12]$. The second effect is that the applied stress changes in the domain wall pinning energies and releases domain walls to move so that the magnetization approaches its anhysteretic state. It is irreversible. Once a domain wall crosses a residual stress peak, which implies rich 
domain wall pinning sites, irreversible magnetization has occurred, so that removal of the stress will not cause the magnetization to revert to its original value. After several torque cycles at a given stress amplitude, most weak domain wall pinning will have been overcome, and the domain walls moved, while domain walls between strong pinning sites could be considered as elastic membranes [10], as shown in Fig. 4. The processes therefore become more and more reversible.

Cullity [9] has discussed the magnetomechanical effect terms of Le Chatelier's principle assuming the process to be reversible. For small reversible changes of magnetization, a thermodynamic relation exists, namely [9]

$$
\left(\frac{\partial \lambda}{\partial H}\right)_{\sigma}=\left(\frac{\partial B}{\partial \sigma}\right)_{H}
$$

The magnetostrictions of polycrystalline iron, cobalt and nickel are shown in Fig.5. Comparison the current results of $\mathrm{Ni}$ with those of $\mathrm{Co}$ and $\mathrm{Fe}$ with their magnetostriction properties are given in Table 1. A relationship between magnetostriction and magnetomechanical effect in response to torsional stress is proposed, which has some similarities to the thermodynamic relation. The sensitivity of magnetization to small applied torsional stress (less than $10 \mathrm{~N} \cdot \mathrm{m}$ ) depends on the piezomagnetic coefficient $d_{33}=\frac{\partial \lambda}{\partial H}$ rather than the saturated magnetostriction at lower magnetic field $(<1000)$. In view of this, a high $d$ coefficient, rather than a high saturation magnetostriction is the critical factor in selecting materials for magnetic torque sensors.

In the demagnetized state there was no apparent surface magnetic field response to applied torque. It could be considered that due to the random distribution of domains inside the sample, the effect of torsional stresses could not lead to any preferred orientation of domain magnetizations.

\section{Conclusions}

Surface magnetic field has been measured as a function of applied torque for rods of pure $\mathrm{Ni}, \mathrm{Fe}$ and $\mathrm{Co}$. The $\mathrm{Ni}$ rod showed substantial changes in surface field even while operating at the remanence. The amplitude of surface axial fields was not a single function 
of the applied torque but depended on the history of torsional strain. The resulting performance can be explained on the basis of stress effects on domain wall motion and pinning. As the number of torque cycles increased, the results became more reversible because by that time domain walls have already been removed from the weak pinning sites, leaving only those attached to stronger sites for which the torque amplitude was unable to remove them.

The surface axial magnetic field of the Ni rod changed approximate linearly with applied torque with a small hysteresis. The sensitivity was $29.7 \mathrm{AN}^{-1} \mathrm{~m}^{-2}$. The response of surface circumferential magnetic field was $18.9 \mathrm{AN}^{-1} \mathrm{~m}^{-2}$. The $\mathrm{Ni}$ rod can thus be thought of as a simple magnetic torque sensor, in which surface magnetic field can be used as a measure of applied torque. No applied field is needed while the sensor is operating. High magnetostriction to applied magnetic field ratio at low field (that is a high $d_{33}$ ) rather than high saturated magnetostriction is recommended as the figure of merit for magnetic torque sensor materials.

\section{Acknowledgements}

This work was supported by the US Department of Energy, Office of Energy Research, Office of Computational and Technology Research, Advanced Energy Projects

Division. The Ames laboratory is operated by the U.S. Department of Energy (DOE) by Iowa State University under contract No. W-74050ENG-82

\section{References}

1. D. C. Jiles, J. Phys. D: Appl. Phys. 28 (1995) 1537-1546.

2. R. A. Beth, W. W. Meeks, Rev. Sci. Instr. 25 (1954), 603.

3. I. J. Garshelis, IEEE Trans. Magn., 28 (1992), 2202.

4. F.Koga and I. Sasada, IEEE Trans. Magn., 31(1995), 3143.

5. H. Hase, R. Shoji, US patent 5442966, 1994.

6. J. F. Herbst, F.E. Pinkerton, J. Magn. and Magn. Mater. 176(1997), 183-196.

7. W. J. Fleming, SAE paper 890482.

8. V. Lemarquand, J. Magn. and Magn. Mater.104-107, pt 2(1992), 1109 
9. B. D. Cullity, Introduction to Magnetic Materials (Addison Wesley, Reading, MA, 1972) p.268.

10. D. C. Jiles, Introduction to Magnetism and Magnetic Materials, Second Edition (Chapman, \& Hall, New York, 1998), p127, p187, 127.

11. I. Garshelis, IEEE Trans. Magn., 2(1974) , 344.

12. M.J. Sablik, D.C. Jiles, IEEE Trans. Magn., 35(1999), 498. 


\section{Figure Captions}

Fig.1 The geometry of the samples tested in the test bed. The torque was applied to the square ends. The surface magnetic fields were measured using a Hall effect sensor.

Fig.2 The amplitude of the axial surface field of a $\mathrm{Ni}$ rod as a function of number of cycles. The boxes represent for the field strength at $-5 \mathrm{~N} \cdot \mathrm{m}$ torque; the stars represent for the field strength at 0 torque; and the dots represent for the field strength at $5 \mathrm{~N} \cdot \mathrm{m}$, at different cycles, respectively. The amplitude of the axial field decreased approximately exponentially with a decay constant time $t 1$ about 3.3 cycles.

Fig.3 (a) The variation of axial surface magnetic field $\mathrm{H}_{\mathrm{a}}$ of a typical $\mathrm{Ni}$ rod changes with applied torque after 12 torque cycles. A small hysteresis can be observed. The sensitivity is $29.7 \mathrm{AN}^{-1} \mathrm{~m}^{-2}$.

(b) The variation of circumferential surface magnetic field $\mathrm{H}_{\mathrm{cm}}$ of the Ni rod changes with applied torque after 12 torque cycles. The sensitivity is $18.9 \mathrm{AN}^{-1} \mathrm{~m}^{-2}$.

Fig.4 Reversible bowing of domain walls.

Fig.5 Bulk magnetostrictions of polycrystalline Iron, Cobalt and Nickel Vs applied magnetic field (after B. D. Cullity [9]). 
Table 1 Comparison of magnetostrictions and MM sensitivities of iron, cobalt and nickel

\begin{tabular}{cccc}
\hline & $\mathrm{Fe}$ & $\mathrm{Co}$ & $\mathrm{Ni}$ \\
\hline $\begin{array}{c}\text { MM Sensitivity } \\
\text { (A/m per N.m) }\end{array}$ & 0.7 & 0.2 & 18.9 \\
$\lambda_{s} \quad\left(10^{-6}\right)$ & 10 & -15 & -35 \\
Piezomagnetic coefficient & Medium & Low & High \\
\hline
\end{tabular}




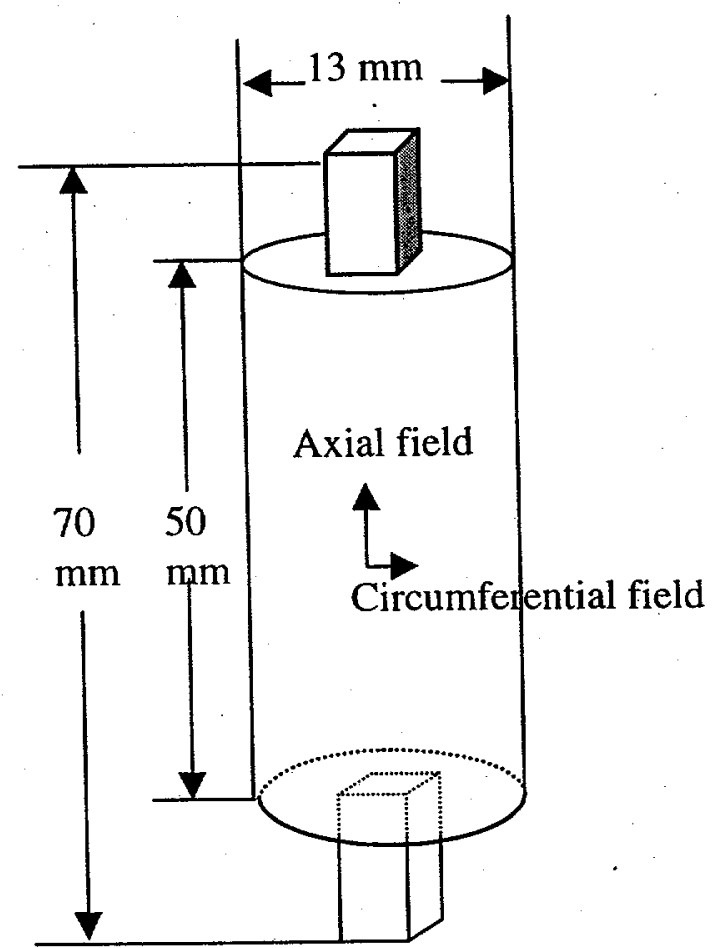

Fig. 1 


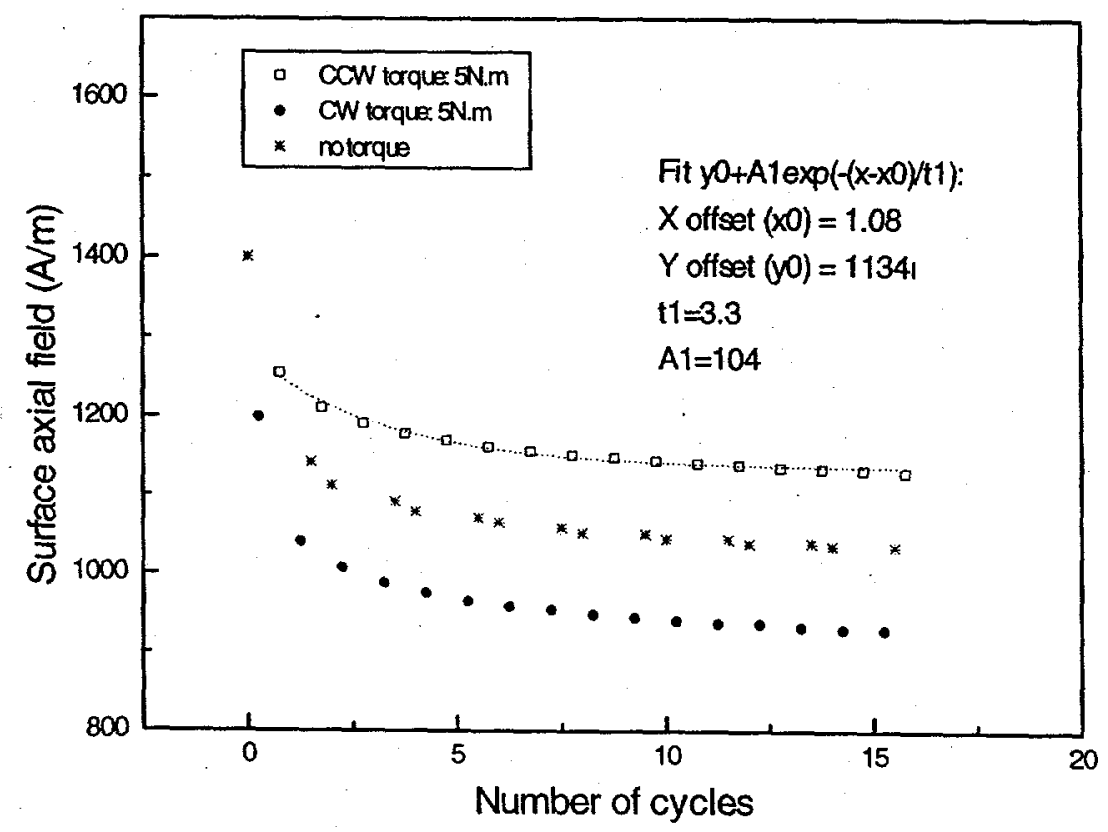

Fig 2 


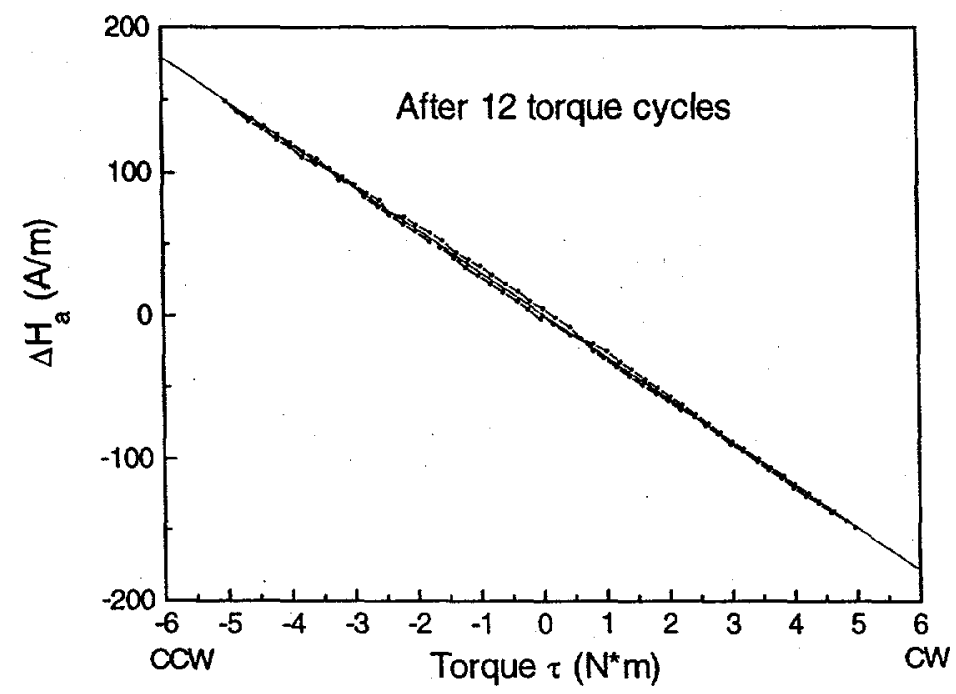

(a)

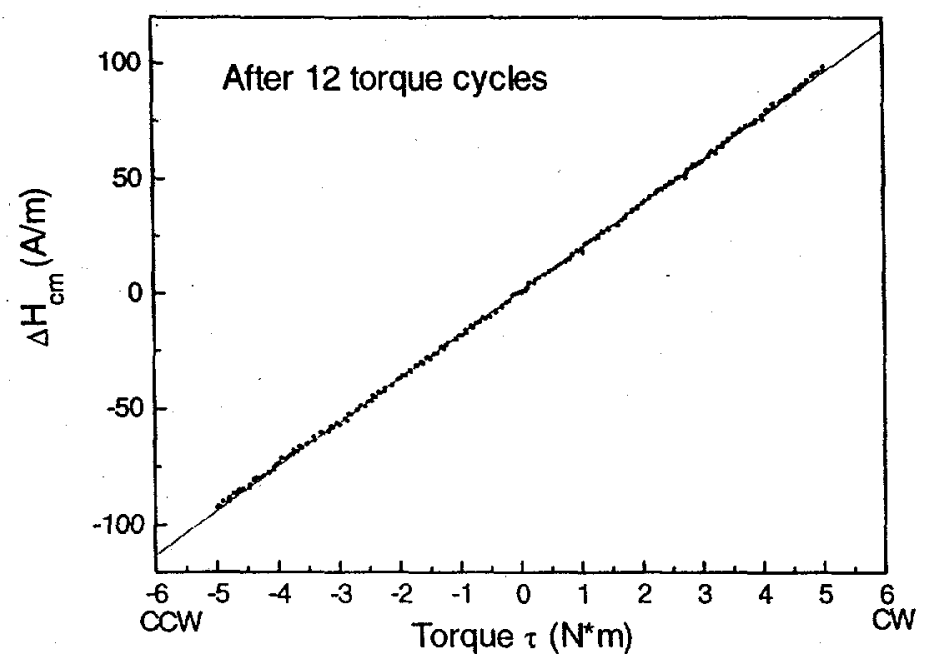

(b)

Fig 3 


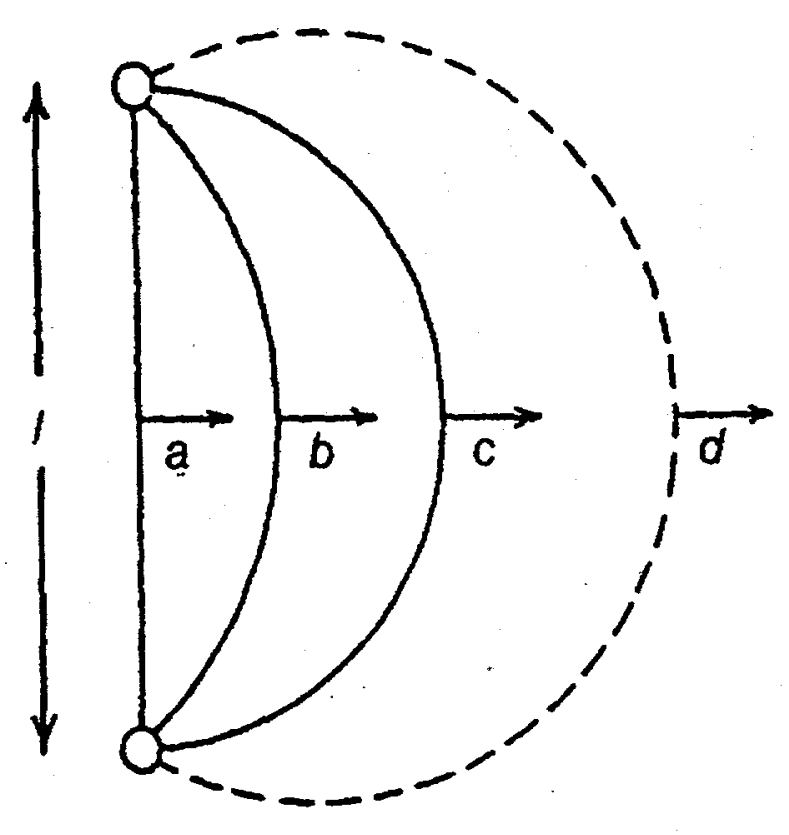

Fig 4 


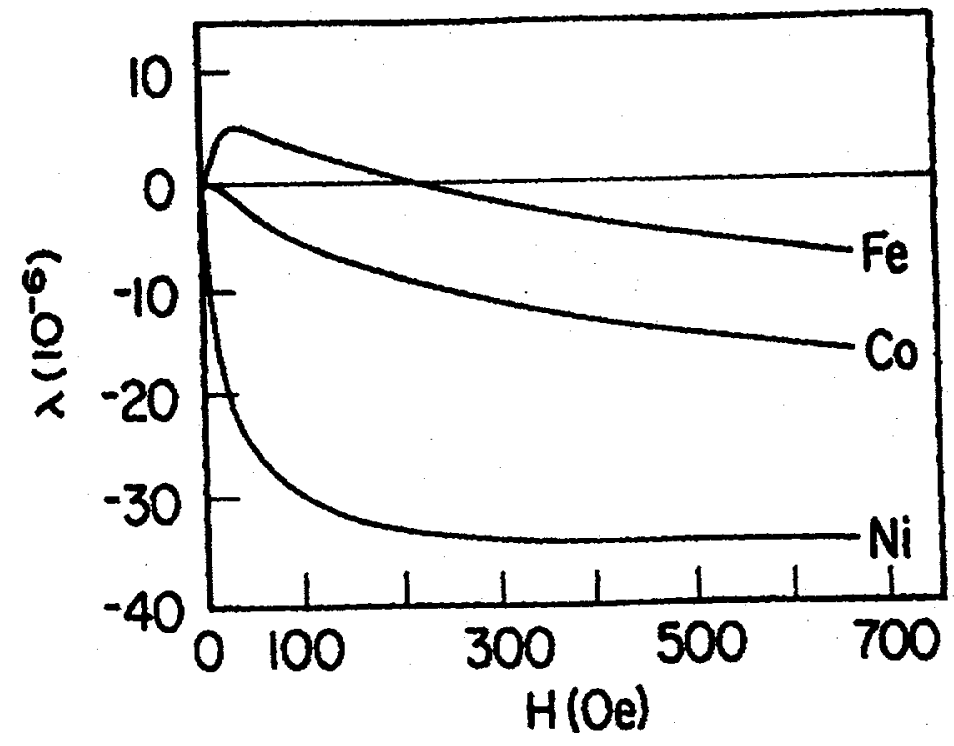

Fig 5 


\title{
Temperature dependence of the magnetomechanical effect in metal- bonded cobalt ferrite composites under torsional strain
}

\author{
Accepted for publication in J. Appl. Phys. \\ Y. Chen ${ }^{1,2}$, J. E. Snyder ${ }^{1}$ K. W. Dennis ${ }^{1}$, R. W. McCallum ${ }^{1,2}$, and D. C. Jiles ${ }^{1,2}$ \\ ${ }^{1}$ Ames Laboratory, ${ }^{2}$ Department of Materials Science \& Engineering, Iowa State University, \\ Ames, Iowa 50011
}

\begin{abstract}
Metal-bonded cobalt ferrite composites are promising candidates for torque sensors and other magnetostrictive sensing and actuating applications. In the present study, the temperature dependence of the magnetomechanical effect in a ring-shape cobalt ferrite composite under torsional strain has been investigated in the temperature range of $-37^{\circ} \mathrm{C}$ to $90^{\circ} \mathrm{C}$. The changes of external axial magnetic field were measured as a function of applied torque. Magnetomechanical sensitivity (mms) of $\Delta \mathrm{H}_{\text {ext }} / \Delta \tau=65 \mathrm{~A} \mathrm{~N}^{-1} \cdot \mathrm{m}^{-2}$ was observed with a magnetomechanical hysteresis $(\mathrm{mmh})$ of $\Delta \tau= \pm 0.62 \mathrm{~N} \cdot \mathrm{m}$ at room temperature $\left(22^{\circ} \mathrm{C}\right)$. These were then measured as a function of temperature. Both decreased as the temperature increased throughout the entire range. The magnetomechanical hysteresis became negligible at temperatures higher than $60^{\circ} \mathrm{C}$, above which there was a linear change in magnetic field with applied torque. These temperature dependences are explained by the changes of magnetostriction, anisotropy, spontaneous magnetization and pinning of domain walls caused by the availability of increased thermal energy.
\end{abstract}

PACS: $75.80,75.60$

Key words: magnetostriction, torsional stress, composites 


\section{Introduction}

Non-contact magnetostrictive torque sensors are candidates for steering sensors for electromechanical steering systems. These will replace the costly and fuel inefficient hydraulic power steering systems currently in use [1-3]. The requirements for the magnetostrictive materials are a high magnetoelastic response, low magnetomechanical hysteresis (mmh), adequate mechanical strength to sustain overtorque of $150 \mathrm{~N} . \mathrm{m}$, and sufficient temperature stability of the magnetomechanical response to allow it to operate over a temperature range of $-40^{\circ} \mathrm{C}$ to $85^{\circ} \mathrm{C}$. Metal-bonded cobalt ferrite composites have been shown to have sufficient magnetoelastic response for torque sensors and other magnetostrictive sensing and actuating applications [4]. These materials have magnetostriction $\lambda_{\mathrm{s}}>200 \times 10^{-6}$ with strain derivative $(\mathrm{d} \lambda / \mathrm{dH})_{\max } \approx 1.3 \times 10^{-9} \mathrm{~A}^{-1} \mathrm{~m}$, and average sensitivity $\Delta \mathrm{H}_{\mathrm{ex}} / \Delta \tau=64 \mathrm{AN}^{-1} \cdot \mathrm{m}^{-2}$ at room temperature $\left(22^{\circ} \mathrm{C}\right)$. In addition, the materials have adequate mechanical properties and corrosion resistance for the application. However, very little is known about the temperature dependence of the magnetomechanical response in these materials. Only a few studies have reported any results on the temperature dependence of magnetostriction or anisotropy of cobalt ferrites [5-7]. Due to the fact that the magnetizations of the sublattices of ferrites have opposite signs and different temperature dependence [8], the temperature dependence of the magnetic properties of ferrites, such as saturation magnetization and anisotropy can be complicated [9]. Metalbonded cobalt ferrite composites are even more complex systems due to the metal additives and different processing from pure cobalt ferrite $[9,10]$. In this study, we report the first results on the temperature dependence of the magnetomechanical response of a cobalt ferrite composite due to torque. 


\section{Experimental Details}

A metal-bonded cobalt ferrite composite $\left(98\right.$ vol\% $\mathrm{CoO} \cdot \mathrm{Fe}_{2} \mathrm{O}_{3}+2$ vol\% $\left.\mathrm{Ag}_{0.97} \mathrm{Ni}_{0.03}\right)$ was pressed and sintered in the form of a ring, then brazed onto a stainless steel shaft. The sample was first magnetized circumferentially to remanence, a configuration that has been used by Garshelis et al. [11-13] In the present study this was achieved using a narrow gap electromagnet. The changes of external axial magnetic field with torque were then measured using a specially designed magnetic torque sensor test bed [14]. The coercivity, remanence, and saturation magnetization were also measured in the temperature range from $-40^{\circ} \mathrm{C}$ to $100^{\circ} \mathrm{C}$, using a Quantum Design MPMS SQUID magnetometer.

Measurements below ambient temperature were performed with the sample immersed in a refrigerated heat-transfer fluid. Ethanol water solution (75 vol\% ethanol + $25 \mathrm{vol} \%$ water) was used as the cooling medium. The torque response was measured at a variety of temperatures from $22^{\circ} \mathrm{C}$ down to $-37^{\circ} \mathrm{C}$. Measurements above ambient temperature were performed with the sample immersed in water which was heated in a separate tank, and circulated using a centrifugal pump. The torque response was measured at each preset point before it was heated to the next higher temperature. During each measurement, the temperature was kept constant to within range of \pm 0.2 deg.C. Finally, the torque response was measured after the sample had cooled back down to $22^{\circ} \mathrm{C}$.

\section{Results}

In this study, magnetomechanical sensitivity $\Delta H_{\text {ext }} / \Delta \tau$, is defined as the slope of the linear portion of the axial field vs. torque plot and the magnetomechanical hysteresis (mmh) 
as the width $\Delta \tau$ in N.m of the magnetomechanical hysteresis loop when $\mathrm{H}_{\mathrm{ext}}$ is zero. The result of torque tests at $-37^{\circ} \mathrm{C}, 22^{\circ} \mathrm{C}$ and $80^{\circ} \mathrm{C}$ are shown in Fig. 1(a-c). The temperature dependence of the magnetomechanical sensitivity and mmh over the range $-37^{\circ} \mathrm{C}$ to $90^{\circ} \mathrm{C}$ are plotted in Fig. 2.

The magnetomechanical sensitivity, $\Delta \mathrm{H}_{\mathrm{ex}} / \Delta \tau$, decreased from $78 \mathrm{AN}^{-1} \mathrm{~m}^{-2}$ at $-37^{\circ} \mathrm{C}$ to $65 \mathrm{AN}^{-1} \mathrm{~m}^{-2}$ at $22^{\circ} \mathrm{C}$ and to $34 \mathrm{AN}^{-1} \mathrm{~m}^{-2}$ at $90^{\circ} \mathrm{C}$. It decreased approximately linearly with increasing temperature as shown in Fig. 2. The decrease in sensitivity was $56 \%$ (on average $0.4 \%$ per deg.C). The $\mathrm{mmh}$ also decreased from $\pm 1.8 \mathrm{~N} \cdot \mathrm{m}$ to $\pm 0.62 \mathrm{~N} \cdot \mathrm{m}$ as the temperature increased from $-37^{\circ} \mathrm{C}$ to $22^{\circ} \mathrm{C}$ and to less than $\pm 0.1 \mathrm{~N}$.m at $60^{\circ} \mathrm{C}$. Over the range $-37^{\circ} \mathrm{C}$ to $60^{\circ} \mathrm{C}$ the hysteresis decreased linearly with temperature giving a decrease of $0.02 \mathrm{~N} . \mathrm{m}$ per deg.C (on average $1 \%$ per deg.C). Interestingly, the mmh remained close to zero as the temperature increased beyond $60^{\circ} \mathrm{C}$, giving a magnetomechanical response $\Delta \mathrm{H}_{\mathrm{ex}} / \Delta \tau$ that was linear and reversible.

The torque response was also measured after the sample had been heated to $90^{\circ} \mathrm{C}$ and cooled back to $22^{\circ} \mathrm{C}$. The sensitivity and $\mathrm{mmh}$ were $48 \mathrm{AN}^{-1} \mathrm{~m}^{-2}$ and $\pm 0.6 \mathrm{~N} \cdot \mathrm{m}$ respectively. As a result of thermal cycling the sensitivity had decreased by about $26 \%$, 'while the mmh returned to its earlier value. These results showed some significant thermal hysteresis in the sensitivity, while the mmh appeared to be unaffected by thermal cycling.

$\mathrm{M}-\mathrm{H}$ hysteresis loops were measured over a similar temperature range as the magnetomechanical response. The coercivity, remanence, and saturation magnetization decreased with temperature in the range from $-40^{\circ} \mathrm{C}$ up to $100^{\circ} \mathrm{C}$, as shown in Fig. 3. The coercivity changed from 14.4 to $4.4 \mathrm{kA} / \mathrm{m}$ (a total of $69 \%$, or $0.5 \%$ per deg.C), the remanent 
magnetization changed from 70 to $26.5 \mathrm{kA} / \mathrm{m}$ (a total of $62 \%$, or $0.44 \%$ per deg.C), and the saturation magnetization changed from 445 to $387 \mathrm{kA} / \mathrm{m}$ (a total of $13 \%$, or $0.09 \%$ per. deg.C)

\section{Discussion}

It is known that the-sensitivity of the magnetomechanical effect is related to magnetostriction [15]. The temperature dependence of magnetostriction has been investigated by Guillaud [7] where it was reported that cobalt ferrite exhibited magnetostrictions of $-270 \times 10^{-6},-190 \times 10^{-6}$, and $-110 \times 10^{-6}$ at $-196,-80$ and $20^{\circ} \mathrm{C}$, respectively. The magnitude of magnetostriction decreased approximately linearly from $-196^{\circ} \mathrm{C}$ to room temperature with an average decrease of magnetostriction of $0.4 \%$ per deg.C. The change of magnetostriction plays an important role in the temperature dependence of the magnetomechanical effect. A decrease in magnetostriction with increasing temperature indicates a decreasing magnetomechanical coupling strength, which could cause a decrease in magnetomechanical response with increasing temperature.

Although the Curie temperature of cobalt ferrite is $520^{\circ} \mathrm{C}$, the saturation magnetization decreases significantly as the temperature increases in the temperature range of $-40^{\circ} \mathrm{C}$ to $100^{\circ} \mathrm{C}$, from about 490 to $427 \mathrm{kA} / \mathrm{m}(85$ to $75 \mathrm{emu} / \mathrm{g}$ ) [9]. Our experimental results confirmed that the metal bonded cobalt ferrite composite exhibits the same behavior. This effect caused the remanent magnetization of the ring-shape ferrite composite to decrease with temperature. This effect was reversible. 
The temperature had another effect on the remanent magnetization when the temperature was raised: specifically it caused partial thermal demagnetization of the sample through release of domain walls from pinning sites. This effect was irreversible. The component of remanence decrease due to the reversible processes at higher temperatures was regained as the sample was cooled back to $22^{\circ} \mathrm{C}$; the component of remanence due to the irreversible process was not regained. Therefore, the thermal energy has only a reversible effect on the measurements at temperatures lower than ambient temperature while having both reversible and irreversible effects on the measurements at temperatures higher than ambient temperature. Since the magnetomechanical effect depends on $M_{R}$, the sensitivity will be dependent on the thermal history from when it was last magnetized.

It is known that torsional stresses on a rod or a ring can be considered to be biaxial stresses, in which the two stress axes are perpendicular to each other and at $45^{\circ}$ to the axis of torsion [16]. The change of axial field is caused by the magnetization of the cobalt ferrite ring rotating towards the compressive stress direction [4]. Therefore, the mmh mainly comes from the anisotropy of the ferrite composite. From the existing literature [9], it was found that the anisotropy of pure $\mathrm{CoO} \cdot \mathrm{Fe}_{2} \mathrm{O}_{3}$ decreased as the temperature increases. The coefficient $\mathrm{K}_{1}$ is $90 \mathrm{KJ} / \mathrm{m}^{3}$ at $90^{\circ} \mathrm{C}, 6.6 \mathrm{KJ} / \mathrm{m}^{3}$ at $200^{\circ} \mathrm{C}$, and is negligible at $280^{\circ} \mathrm{C}$. The coercivity of the metal bonded cobalt ferrite composite of the present study, which would depend strongly on anisotropy, was likewise observed to decrease linearly as the temperature increased from -40 to $100^{\circ} \mathrm{C}$. Thus the temperature dependence of the $\mathrm{mmh}$ is caused principally by the temperature dependence of the anisotropy. The metal additives $(\mathrm{Ag} / \mathrm{Ni})$ and the bonding treatment of the cobalt ferrite composite could also change the 
anisotropy and the temperature dependence of anisotropy. The coercivity $(\sim 8 \mathrm{kA} / \mathrm{m})$ of the present metal-bonded cobalt ferrite composite is much lower than that reported for cobalt ferrite at $22{ }^{\circ} \mathrm{C}(\sim 160 \mathrm{kA} / \mathrm{m})[7]$.

It is interesting to note that the mmh effectively disappears above $60^{\circ} \mathrm{C}$ while a sufficient sensitivity still exists at that temperature to give a good signal/noise ratio (about $50 \mathrm{AN}^{-1} \mathrm{~m}^{-2}$ ). Therefore, it is should be possible to adjust the temperature dependent anisotropy of cobalt ferrite composites by adjusting the levels of metallic additives $(\mathrm{Ag} / \mathrm{Ni} / \mathrm{Co})$ in order to obtain linear torque response at room temperature.

\section{Conclusions}

The temperature dependence of the magnetomechanical effect under torsional strain in metal-bonded cobalt ferrite composite was investigated. The sensitivity $\Delta \mathrm{H}_{\text {ext }} / \Delta \tau$ changed from $78 \mathrm{AN}^{-1} \mathrm{~m}^{-2}$ at $-37^{\circ} \mathrm{C}$ to $34.2 \mathrm{AN}^{-1} \mathrm{~m}^{-2}$ at $90^{\circ} \mathrm{C}$ while the magnetomechanical hysteresis $\Delta \tau$ decreased from $\pm 1.8 \mathrm{~N} \cdot \mathrm{m}$ at $-37^{\circ} \mathrm{C}$ to negligible above $60^{\circ} \mathrm{C}$. The effects of temperature on magnetomechanical effect under torque at remanence can be described in terms of changes in four properties of the material: magnetostriction, anisotropy, spontaneous magnetization and pinning of domain walls. Metal-bonded cobalt ferrite composites showed linear magnetomechanical response to torque at high temperatures. This should be achievable at lower temperatures by changing the temperature dependence of the anisotropy of the material through the use of metallic additives. 


\section{References}

1. R.A. Beth, and W.W. Meeks, Rev. Sci. Instr. 25, 603 (1954).

2. W.J. Fleming, Society of Automotive Engineers, Paper No. 900264, 1990.

3. F.E. Pinkerton, T.W. Capehart, J.F. Herbst, E.G. Brewer, C. B. Murphy, Appl. Phys. Lett. 70, 2601 (1997).

4. Y.Chen, J. E. Snyder, C.R. Schwichtenberg, K. W. Dennis, R. W. McCallum, and D. C. Jiles, IEEE Trans. Mag., 35, 3652, 1999.

5. R.M. Bozorth, E.F. Tilden, and A.J. Williams, Phys. Rev., 99 1788, (1955).

6. P.E. Tannenwald, Phys. Rev. 99, 463-464 (1955).

7. C. Guillaud, Rev. Modern. Phys., 25, 64, (1953).

8. E. Du T. de Lacheisserie, Magnetostriction, p267, CRC Press, New York, 1993.

9. J. Smit, and H.P.J. Wijn, Ferrites, John Wiley \& Sons, New York, 1959.

10. R. Valenzuela, Magnetic Ceramics, Cambridge University Press, 1994.

11. I.J.Garshelis, IEEE Trans., Mag. 28, 2202, (1992).

12. I.J.Garshelis and C.R.Conto, J.Appl. Phys., 79, 4756, (1996).

13. I.J.Garsheis and C.A.Jones, J.Appl.Phys., 85, 5468, (1999).

14. Y.Chen, J.E.Snyder, C.R.Schwichtenberg, K.W.Dennis, R.W.McCallum and D.C.Jiles. Paper HF-09, 43rd Annual Conference on Magnetism and Magnetic Materials, Miami, November 1998.

15. D.C.Jiles, J.Phys.D (Appl.Phys.), 28, 1537, (1995).

16. M.J. Sablik, and D.C. Jiles, IEEE Trans. Magn., 35, 498, (1999). 


\section{Figure captions}

Fig. 1 The magnetomechanical torque responses at three typical temperatures. In this study, the magnetomechanical sensitivity (mms) is defined as the slope of the linear portion of the external axial field vs torque plot $\mathrm{dH}_{\mathrm{ex}} / \mathrm{d} \tau$. The magnetomechanical hysteresis (mmh) is defined as the width of the field versus torque loop at zero field. (a) $-37^{\circ} \mathrm{C}: \mathrm{mms}=78 \mathrm{AN}^{-}$ ${ }^{1} \mathrm{~m}^{-2} ; \mathrm{mmh}= \pm 1.8 \mathrm{~N} \cdot \mathrm{m} \cdot$ (b) $22^{\circ} \mathrm{C}: \mathrm{mms}=65 \mathrm{AN}^{-1} \mathrm{~m}^{-2}, \mathrm{mmh}= \pm 0.62 \mathrm{~N} \cdot \mathrm{m} \cdot$ (c) $80^{\circ} \mathrm{C}: \mathrm{mms}=$ $38.4 \mathrm{AN}^{-1} \mathrm{~m}^{-2}, \mathrm{mmh}$ is negligible.

Fig. 2 The temperature dependence of the magnetomechanical effect of the ring-shape cobalt ferrite composite under torsional strain in the temperature range of $-37^{\circ} \mathrm{C}$ to $90^{\circ} \mathrm{C}$. The empty squares represent magnetomechanical sensitivity and the filled squares represent magnetomechanical hysteresis.

Fig. 3 The temperature dependence of coercivity, remanent magnetization and saturation magnetization of metal-bonded cobalt ferrite composite. All three properties are normalized by the values at $-40^{\circ} \mathrm{C}$. 

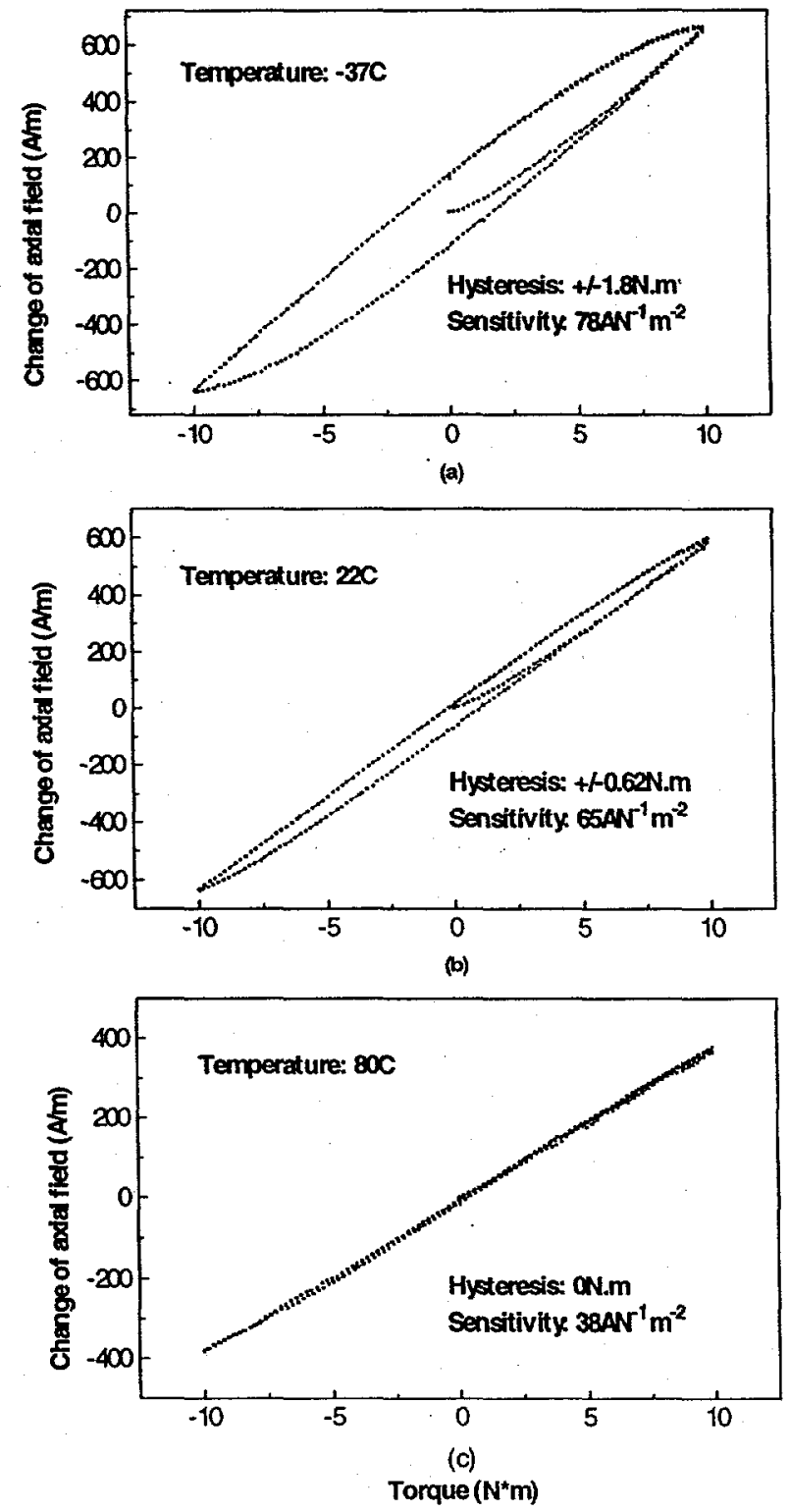

Fig.1 


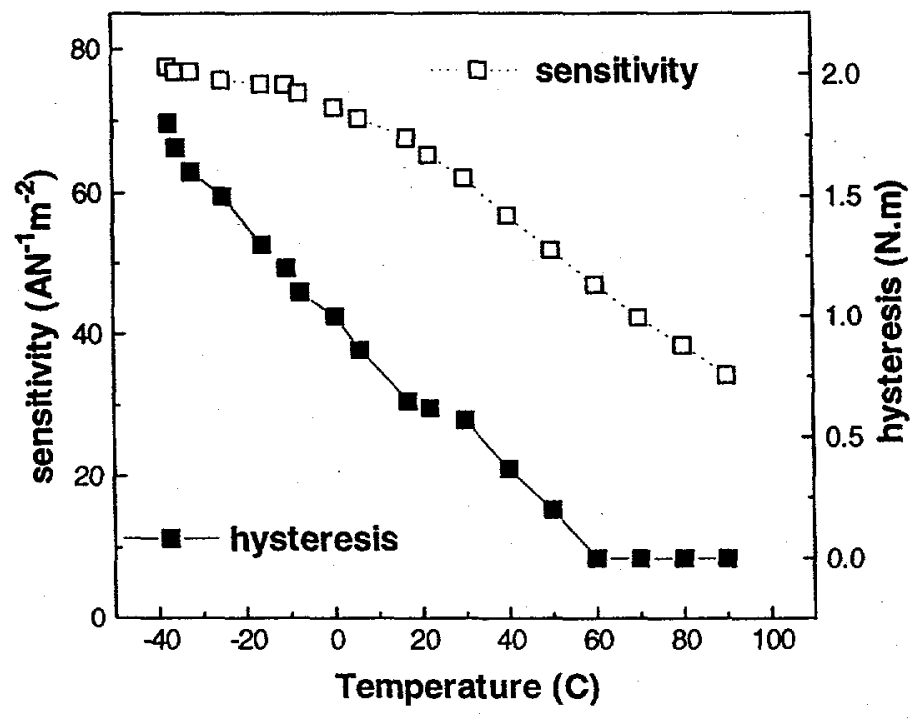

Fig. 2 


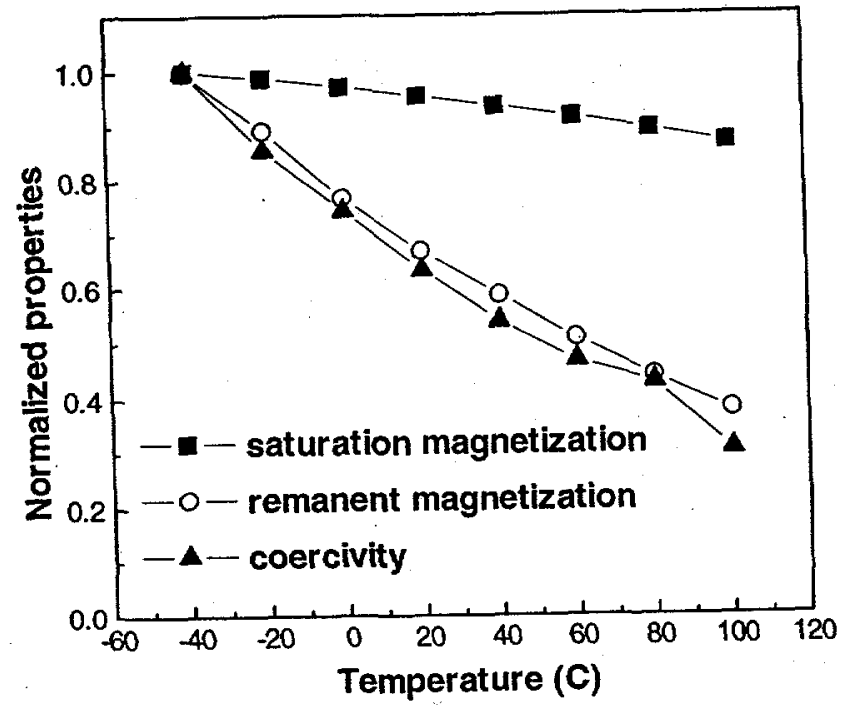

Fig. 3 
MODIFIED LAW OF APPROACH FOR THE MAGNETOMECHANICAL MODEL

Presented at the Review of Progress in Quantitative Nondestructive Evaluation, Montreal, Canada, July 26-30, 1999.

\author{
M.J. Sablik \\ Southwest Research Institute \\ P.O. Drawer 28510 \\ San Antonio, TX 78228-0510 \\ Y. Chen and D.C. Jiles \\ Ames Laboratory \\ Iowa State University \\ Ames, IA 50011
}

\title{
INTRODUCTION
}

Computer modeling and simulation of materials behavior is becoming increasingly important in NDE. This work is concerned with improving the modeling of magnetization changes under constant magnetic field and varying stress, ie. magnetization changes known as $\mathrm{H}-\sigma$ processes. Viable modeling of these processes can allow us to predict the behavior of magnetic materials under stress.

\section{ORIGINAL MAGNETOMECHANICAL MODEL OF JILES}

The original model is based on what is termed the law of approach, which says that during $\mathrm{H} \sigma$ processes, the rate of change, $\mathrm{dM}_{\mathrm{i}} / \mathrm{dW}$, of the irreversible magnetization with respect to change in elastic energy density as a result of varying stress $\sigma$ is proportional to the deviation of the irreversible magnetization $M_{i}$ from the anhysteretic magnetization $M_{a}$. Thus, algebraically, the law of approach states that

$$
d M_{i} / d W=(1 / \xi)\left(M_{a}-M_{i}\right),
$$

where $\xi$ is a constant which is a property of the material and is a kind of "relaxation" coefficient. The elastic energy density is taken as $\mathrm{W}=\sigma^{2} / 2 \mathrm{E}$, where $\mathrm{E}$ is the elastic modulus, and hence

$$
d W=(\sigma / E) d \sigma
$$

Notice that eq. (1) says that the irreversible magnetization changes significantly when the deviation $\left(\mathbf{M}_{\mathbf{a}}-\mathbf{M}_{\mathbf{i}}\right)$ is large, and less when the deviation is small. Since the general experimental behavior [3] is such that the magnetization changes in such a way as to 
approach the anhysteretic during $\mathrm{H} \sigma$ processes, the above law of approach seems to be a quite reasonable interpretation of the data.

The rest of the model [1] can be written out as follows

$$
M=M_{i}+c\left(M_{a}-M_{i}\right)
$$

where $M$ is total magnetization. The first term on the right hand side, the irreversible magnetization, is due to magnetization ensuing from domain wall pinning and unpinning. The second term is due to domain wall bowing, where $c$ is a material constant [4]. It then follows that

$$
\frac{d M}{d W}=\left(\frac{d M_{i}}{d W}\right)(1-c)+c \frac{d M_{a}}{d W}
$$

and using eq. (1) and (2),

$$
\frac{d M}{d \sigma}=\frac{\sigma(1-c)}{\varepsilon^{2}}\left(M_{a}-M_{i}\right)+c \frac{d M_{a}}{d \sigma},
$$

where the constant $\epsilon$ is defined as

$$
\varepsilon=(E \xi)^{1 / 2}
$$

Similarly,

$$
\frac{d M_{i}}{d \sigma}=\frac{\sigma}{\varepsilon^{2}}\left(M_{a}-M_{i}\right)
$$

To determine the curve followed by the magnetization from $\sigma_{1}$ to $\sigma_{2}$ in steps of $d \sigma$, one obtains $d M_{i}$ and $d M$ from eqs. (5) and (7) at each step $d \sigma$ and $\mathrm{dM}_{\mathrm{a}}$ from

$$
M_{a}=M_{s} \mathfrak{E}\left(H_{c}(M) / a\right)
$$

where $M_{s}$ is the saturation magnetization, $a$ is the effective field scaling constant, the function _ $(x)=\operatorname{coth} x-1 / x$ is the Langevin function, and $H_{e}(M)$ is the effective field given by

$$
H_{\varepsilon}(M)=H+\alpha M+\left(3 \sigma / 2 \mu_{0}\right)(d \lambda / d M)
$$

where $\alpha$ is the domain coupling constant and where the third term was added by Sablik et al. $[5,6]$ to represent the coupling of stress to the effective field, where $\lambda$ is the bulk 
magnetostriction and $\mu_{0}$ is the permeability of free space. In his magnetomechanical model [1], Jiles writes the following for the magnetostriction

$$
\lambda=\gamma_{1}(\sigma) M^{2}-\gamma_{2}(\sigma) M^{4}+\ldots \ldots
$$

where

$$
\begin{aligned}
& \gamma_{1}(\sigma)=\gamma_{11}+\gamma_{12} \sigma+\ldots \ldots \\
& \gamma_{2}(\sigma)=\gamma_{21}+\gamma_{22} \sigma+\ldots \ldots
\end{aligned}
$$

The magnetostriction is represented in even powers of the magnetization because of the tendency of plots of $\lambda$ vs $M$ to exhibit reflection symmetry about the ordinate axis. $[5,6]$

Two alternative ways of writing $M_{a}$ are

$$
M_{a}=M_{s} £\left(H_{c}\left(M_{a}\right) / a\right)
$$

or

$$
M_{a}=M_{s} \mathfrak{f}\left(H_{c}\left(M_{i}\right) / a\right)
$$

In the original magnetomechanical model of Jiles [1], eq. (12b) is used instead of eq. (8). However, it is our experience that regardless of which $M$ one uses for $H_{e}(M)$, the equations lead to magnetization curves of essentially the same behavior, but with the behavior being the same only if $\epsilon$ is set to different ranges of $\in$ for each of the types of $M$. That is, the equations can be recast in terms of either $M_{i}$ or $M_{a}$, but this leads to different values of $\in$.

\section{THE PROBLEM}

The model was tried on a process in which one follows the sequence: (1) vary $\mathrm{H}$ from zero to $H$; (2) vary $\sigma$ from zero to $+\sigma_{\max }$; (3) vary $\sigma$ from $+\sigma_{\max }$ to $-\sigma_{\max }$; (4) vary $\sigma$ from $-\sigma_{\max }$ to $+\sigma_{\max } ;(5)$ repeat steps (3) and (4) over and over; in our case, five times in all. 


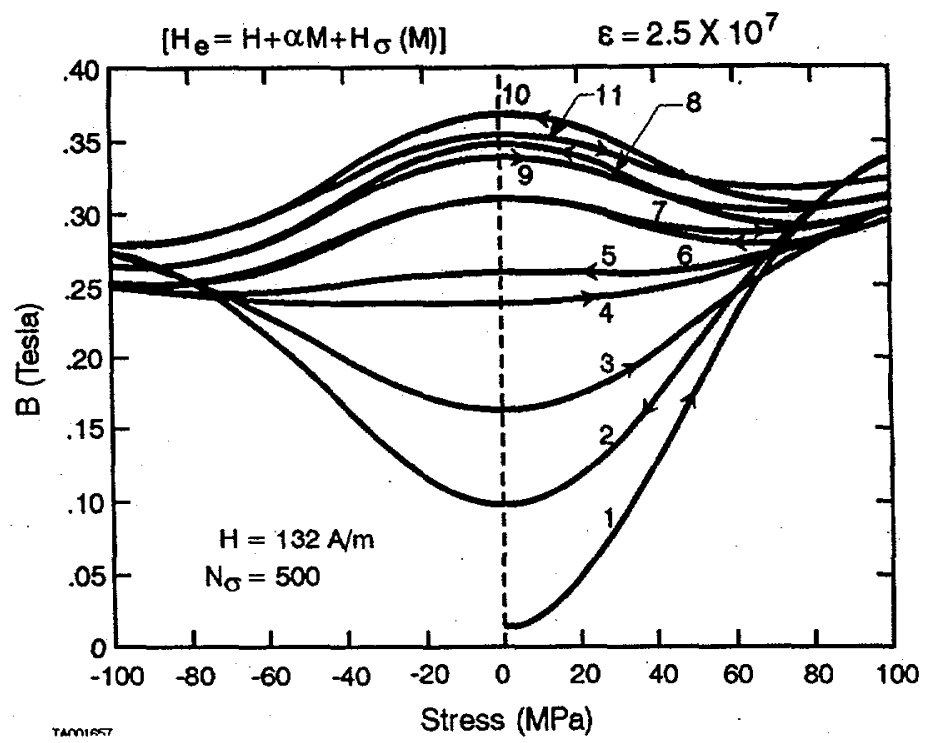

Fig. 1. Variation of the magnetization according to the original magnetomechanical model [1] after repeated stress cycling at constant field $\mathrm{H}$. The numbers represent the magnetization curve obtained during each succeeding leg of the stress variation.

From Fig. 1, it is seen that the computed magnetization near zero stress appears to increase in unlimited fashion rather than ultimately tending to a limiting hysteretic pattern, as seen experimentally. [7-9] In the computation shown in Fig. $1, \epsilon=2.5 \times 10^{7} \mathrm{~Pa}, \mathrm{M}_{\mathrm{s}}=$ $1.71 \times 10^{6} \mathrm{~A} / \mathrm{m}, \alpha=0.0011, \mathrm{a}=900 \mathrm{~A} / \mathrm{m}, \mathrm{c}=0.1$, and $\mathrm{k}=2000 \mathrm{~A} / \mathrm{m}$, where the pinning constant $\mathrm{k}$ is used in computing the magnetization in step (1) of the process when field is varying [4]. Also, $\gamma_{11}=0.5 \times 10^{-18} \mathrm{~m}^{2} / \mathrm{A}^{2}, \gamma_{21}=-2.0 \times 10^{-30} \mathrm{~m}^{4} / \mathrm{A}^{4}, \gamma_{12}=1.0 \times 10^{-26}$ $\left(\mathrm{m}^{2} / \mathrm{A}^{2}\right) / \mathrm{Pa}$, and $\gamma_{22}=-6.5 \times 10^{-38}\left(\mathrm{~m}^{4} / \mathrm{A}^{4}\right) / \mathrm{Pa}$. Curves similar to Fig. 1 are obtained if eqs. (12a) or (12b) are used instead of eq. (8). If eq. (12b) is used, a suggested value of $\in$ for seeing similar behavior is $\epsilon=2.0 \times 10^{7} \mathrm{~Pa}$, whereas if eq. (12a) is used, $\epsilon=2.5 \times 10^{7} \mathrm{~Pa}$ also produces behavior similar to Fig. 1 except that increase in magnetization about $\sigma=0$ is a little more pronounced in successive processes.

\section{MODIFICATION OF MODEL}

Two possible approaches to generalizing the model seem reasonable, as given below:

\section{$\underline{\text { Remedy \#1 }}$}

It is proposed to use two different relaxation constants. $\xi_{a}$ and $\xi_{r}$, with $\xi_{r}>\xi_{a}$ (i.e., $\in_{a}$ and $\epsilon_{\mathrm{r}}$, with $\epsilon_{\mathrm{r}}>\epsilon_{\mathrm{a}}$ ).

The physical concept for this is that stress application (a) is different from stress release (r). Stress application (i.e. increasing stress) essentially "reshapes" domain boundaries, causing some irreversible wall movement and some bowing of domain walls. 
Of the walls that move, some get so strongly pinned that they do not move on stress release (i.e. decreasing stress). Wall reshaping is therefore different on stress release, implying a different relaxation constant, $\xi$ or $\in$, for the release process.

Fig. 2 shows different variations of the magnetization after stress cycling, using this revised model and different sets of $\epsilon_{\mathrm{a}}$ and $\epsilon_{\mathrm{r}}$. In some cases, the limiting stress hysteresis loop for magnetization is approached very quickly. In others, it is approached more slowly or not quite reached. For the calculations of Fig. 2, eq. (8) is used and the same constant parameters are used as previously, except for the choice of $\epsilon_{\mathrm{a}}$ and $\epsilon_{\mathrm{r}}$ in place of $\epsilon$.

Clearly, the behavior seen experimentally can now be produced by the revised version of the model, at least qualitatively.
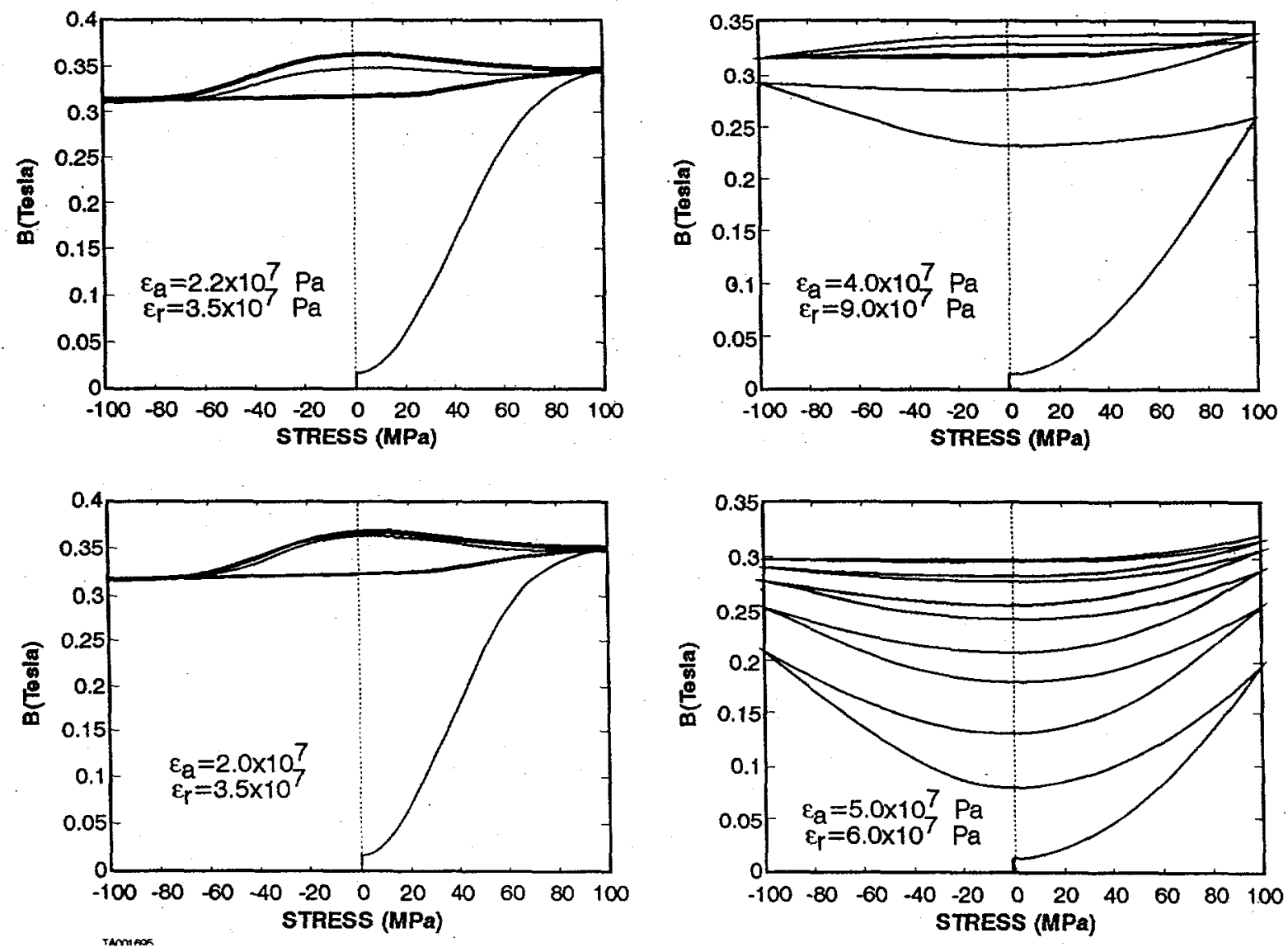

Fig. 2. Variation of the computed magnetization with stress cycling using revised model \#1 and different sets of $\epsilon_{\mathrm{a}}$ and $\epsilon_{\mathrm{r}}$, as indicated. 
In this case, the law of approach is rewritten as

$$
\frac{d M_{i}}{d \sigma}=\frac{\sigma-\sigma_{t}}{\varepsilon^{2}}\left(M_{a}-M_{i}\right),
$$

where $\sigma_{t}$ is a "turning point stress", i.e. it is the stress that existed the last time the sign of the changing stress was changed. Hence, referring to our process under consideration, the following obtains: (1) for stress varying from zero to $+\sigma_{\max }, \sigma_{t}=0$; (2) for stress varying from $+\sigma_{\max }$ to $-\sigma_{\max }, \sigma_{t}=+\sigma_{\max }$; (3) for stress varying from $-\sigma_{\max }$ to $+\sigma_{\max }, \sigma_{t}=\sigma_{\max }$. Eq. (13) is formulated in a manner similar to the way in which minor loops for $\mathrm{M}$ vs $\mathrm{H}$ variations are formulated. [10] One problem with its formulation is that it would imply that

$$
d W=\left[\left(\sigma-\sigma_{t}\right) / \varepsilon\right] d \sigma,
$$

which seems hard to justify physically. However, our experience is that hysteretic physical processes always need to take into account the history of application of control variable, including reversal points. In this case, the control variable is asserted to be the stress.

The rationale for using eq. (13) came from the following observation of the original model behavior. With

$$
d M_{i}=\frac{\sigma d \sigma}{\varepsilon^{2}}\left(M_{a}-M_{i}\right),
$$

then if $\sigma$ is decreasing (i.e. $d \sigma<0)$ and $\sigma$ is positive $(\sigma>0)$, then if $M_{a}>M_{i}$, it follows that $\mathrm{dM}_{\mathrm{i}}$ is negative and $\mathrm{M}_{\mathrm{i}}$ decreases and moves further from $\mathrm{M}_{\mathrm{a}}$, which was not what was intended for the law of approach. With the turning point inserted as in eq. (13), then

$$
d M_{i}=\frac{\left(\sigma-\sigma_{i}\right) d \sigma}{\varepsilon^{2}}\left(M_{a}-M_{i}\right),
$$

and if $\sigma$ is decreasing $(\mathrm{d} \sigma<0)$ from $\sigma_{\max }\left(\mathrm{viz}, \sigma_{\mathrm{t}}\right)$., then with $\sigma>0$ and $\mathrm{M}_{\mathrm{a}}>\mathrm{M}_{\mathrm{i}}$, it follows that $\mathrm{dM}_{\mathrm{i}}$ is positive and $\mathrm{M}_{\mathrm{i}}$ continues to approach $\mathrm{M}_{\mathrm{a}}$. The reformulation sounds like the "right" law of approach despite it being hard to physically justify eq. (14).

Numerically, we find the behavior seen in Fig. 3, where this time different cases are chosen for $\epsilon$. 

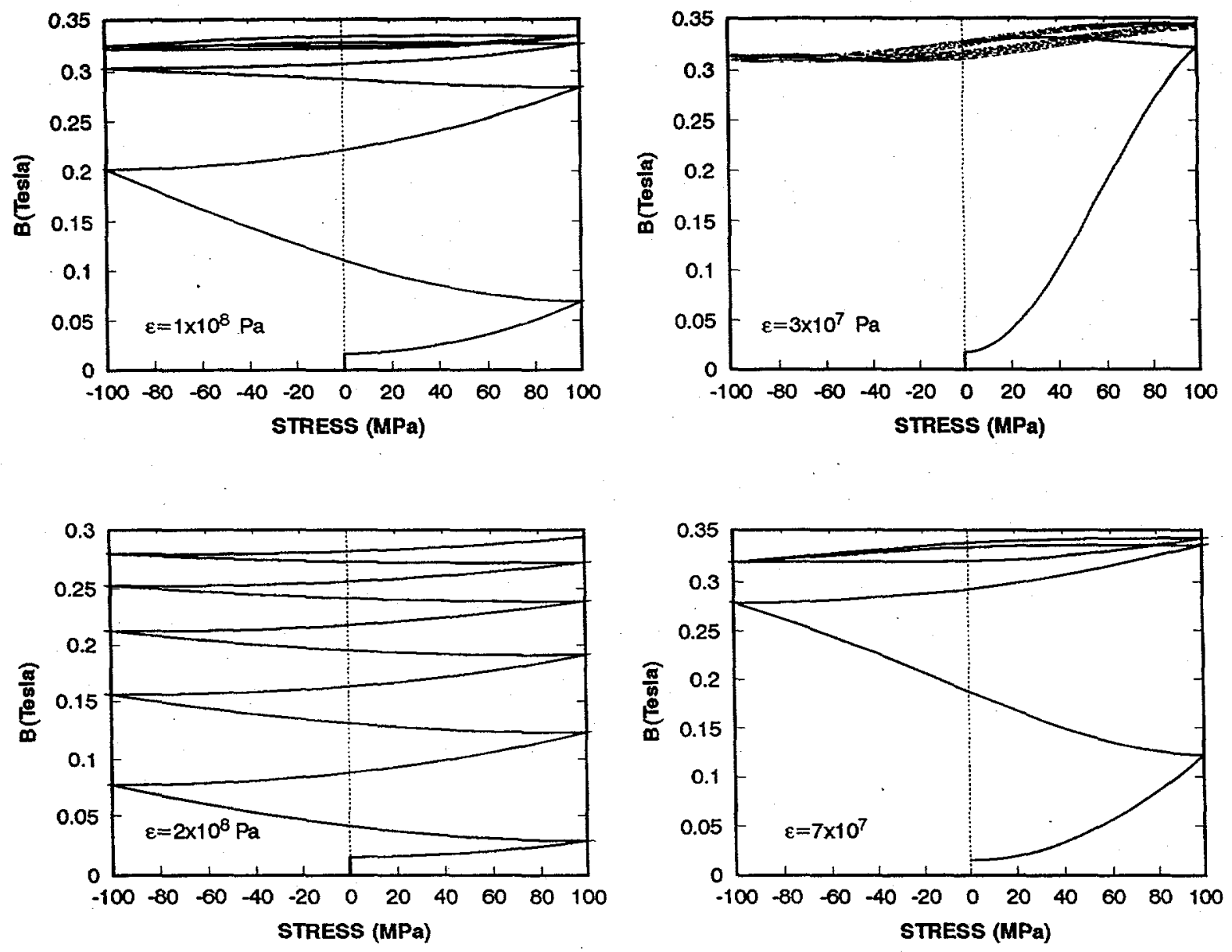

Fig. 3. Various cases of the computed magnetization with stress cycling using revised model $\# 2$ and different values for $\epsilon$.

We note that a limiting stress hysteresis loop is again approached using this second revised model. The limiting loop does not show quite as much hysteresis as the first revised model. However, it is still possible to produce cases where the magnetization quickly goes to the limiting behavior, more slowly approaches the limiting behavior, or does not quite reach the limiting behavior after 5 cycles. Thus, qualitatively, the second model also exhibits agreement with behavior seen experimentally.

\section{CONCLUSIONS}

We have found two reformulations of the magnetomechanical model that appear to give the "correct" physical behavior for magnetization variation with stress at constant field, but have not yet found a way to select one over the other. Thus, this is a work in progress. 
Further combinations of experimental work and computer simulation should enable us to decide which of these two approaches is correct.

\section{ACKNOWLEDGMENTS}

We acknowledge the funding support of the U.S. Department to Energy, Office of Energy Research, Office of Computational and Technology Research, Advanced Energy Project Division.

\section{REFERENCES}

1. D.C. Jiles, J. Phys. D 28, 1537 (1995).

2. D.C. Jiles, in Review of Progress in QNDE, vol. 16, eds. D.O. Thompson and D.E. Chimenti (Plenum, N.Y., 1997), p. 1739.

3. D.C. Jiles and D.L. Atherton, J. Phys. D 17, 1267 (1984).

4. D.C. Jiles and D.L. Atherton, J. Magn. Mater. 61, 48 (1986).

5. M.J. Sablik and D.C. Jiles, IEEE Trans. Magn. 29, 2113 (1993).

6. M.J. Sablik, G.L. Burkhardt, H. Kwun, and D.C. Jiles, J. Appl. Phys. 63, 3930 (1988).

7. D.P. Craik and R.J. Fairholme, J. de Physique Colloq. C1, 32, 681 (1971).

8. D.L. Atherton and J.A. Szpunar, IEEE Trans. Magn. 22, 514 (1986).

9. I.M. Robertson, IEEE Trans. Magn. 29, 2078 (1993).

10. D.C. Jiles, IEEE Trans. Magn. 28, 2602 (1992). 


\title{
THE MAGNETOMECHANICAL EFFECT UNDER TORSIONAL STRESS AND THE LAW OF APPROACH IN A CO FERRITE COMPOSITE
}

\author{
Submitted to Intermag'2000 Conference, Toronto, Canada, April 9-13, 2000. \\ Y. Chen and D.C. Jiles \\ Ames Laboratory, and Department of Materials Science and Engineering \\ Iowa State University, Ames, Iowa 50011
}

\section{Introduction}

Non-contact magnetic torque sensors are considered suitable means of transferring information between computers/processors and mechanical systems such as robotic systems and automobile applications. The magnetomechanical effect under torsional stress, also called the inverse Wiedemann effect, is now receiving increased attention because of its relevance to magnetic torque sensors [1-3]. Metal bonded Co ferrite composites exhibit promising performance as a torque sensor material [3]. In the present work, the law of approach is used to model the inverse Wiedemann effect. The modeled results are in good agreement with the experimental results, shown in Fig.1.

\section{Experimental}

A Co ferrite composite was sintered in the form of rings that were brazed onto a stainless steel shaft. The sample was magnetized circumferentially to remanence before being subjected to torque. The changes of external axial magnetic fields were measured as a function of applied torque in the range of $\pm 10 \mathrm{~N} \cdot \mathrm{m}$. The experimental result is shown in Fig.l with a sensitivity of $65 \mathrm{~A} / \mathrm{m}$ per $\mathrm{N} \cdot \mathrm{m}$ and a hysteresis $\pm 0.5 \mathrm{~N} \cdot \mathrm{m}$.

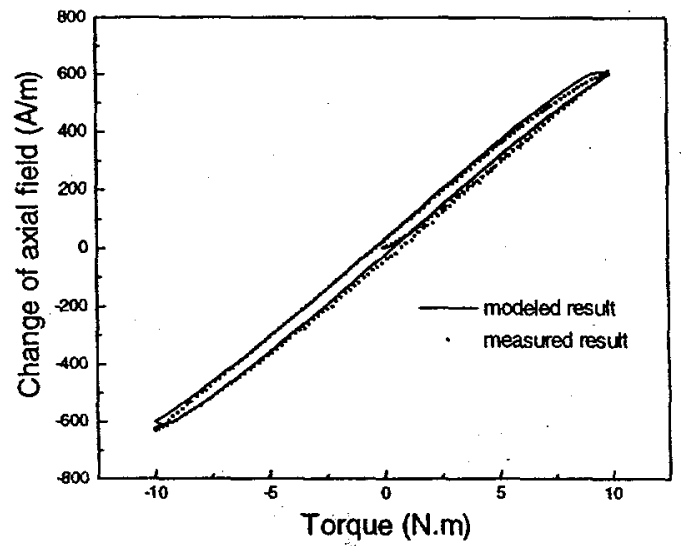

Fig. 1 Comparison of modeled and measured result

Modeling

The law of approach to the anhysteretic magnetization was extensively used to model the magnetomechanical effect $[2,4,5]$. Applying the law of approach to the magnetomechanical effect, there are three factors that determine the change of the magnetization of material [4].

1. How far the magnetization is above or below the anhysteretic state

2. How sensitive this displacement is to stress (the rate of approach)

3. How the anhysteretic magnetization changes with.stress

The third factor was considered using an effective field representation of stress. The effective field of uniaxial stress is expressed by

$$
H_{\sigma}=\frac{1}{\mu_{0}} \frac{\partial E_{o}}{\partial M}=\frac{3}{2} \frac{\sigma}{\mu_{0}}\left(\frac{\partial \lambda}{\partial M}\right)_{\sigma}\left(\cos ^{2} \theta-v \sin ^{2} \theta\right)
$$


where $\lambda=$ the magnetostriction, $\sigma=$ applied stress, $\theta=$ the angle between the stress and magnetization, and $v=$ Poisson's ratio. Applying the law of approach, the change of magnetization with stress is given by

$$
\mathrm{dM}=\frac{\sigma}{\varepsilon^{2}}(1-\mathrm{c})\left(\mathrm{M}_{\mathrm{an}}-\mathrm{M}_{\mathrm{in}}\right) \mathrm{d} \sigma+\mathrm{c} \cdot \mathrm{d} \mathrm{M}_{\mathrm{an}}
$$

where $\mathrm{c}$ is the reversibility and $\varepsilon$ reflects the rate of approach, the anhysteretic function $\mathrm{M}_{\mathrm{an}}$ is represented by the Langevin function using the total effective field.

If a rod (or a ring) is subjected to torque $\tau$ in the plane normal to its axis, the torsion can be considered as creating two orthogonal stresses at $\pm 45^{\circ}$ from the axis. The stress can be calculated by

$$
\sigma=2 \tau \mathrm{r} / \pi \mathrm{R}^{4}
$$

where $\mathrm{R}$ is the outer radius of the rod/ring and $\mathrm{r}$ the distance from the axis. In this case, the effective field due to stress can be obtained [2]

$$
H_{\sigma}=\frac{3 \sigma}{2 \mu_{0}}\left(\left(\frac{\partial \lambda(\sigma)}{\partial \mathrm{M}}\right)\left(\cos ^{2} \theta-v \sin ^{2} \theta\right)-\left(\frac{\partial \lambda(-\sigma)}{\partial \mathrm{M}}\right)\left(\sin ^{2} \theta-v \cos ^{2} \theta\right)\right)
$$

where $\lambda(\sigma), \lambda(-\sigma)$ are the magnetostriction under tension and compressive stress respectively, and $\theta$ is the angle between the magnetization and the tensile stress. Applying the law of approach (Eq. 2), the change of magnitude of magnetization was modeled.

Besides changing its amplitude, the magnetization of the material rotates with respect to torque as well. Garshelis [1] and Sablik [2] derived formulae to predict the rotation of magnetization under the action of a torque assuming thermodynamic equilibrium, i.e. energy minima. In the present work, considering the stress dependence of magnetostriction, the equilibrium deviation of magnetization from its initial direction is expressed by the angle

$$
\phi_{c}=\frac{1}{2} \tan ^{-1} \frac{\frac{3}{2} \sigma \lambda(\sigma)\left(\operatorname{Cos}^{2} \theta-v \operatorname{Sin}^{2} \theta\right)-\frac{3}{2} \sigma \lambda(-\sigma)\left(\operatorname{Sin}^{2} \theta-v \operatorname{Cos}^{2} \theta\right)}{\mathrm{K}_{\mathrm{u}}+\mu_{0} \mathrm{~N}_{\mathrm{d}} \mathrm{M}^{2} / 2}
$$

where $K_{u}$ is the anisotropy and $N_{d}$ is the demagnetizing factor. However, the magnetomechanical effect is a kinetic process other than an equilibrium process. Therefore, the law of approach of magnetization can also be extended to the rotation of magnetization.

$$
\mathrm{d} \phi=\frac{1}{1-\mathrm{c}}\left[\mathrm{cd}\left(\phi_{\mathrm{e}}-\phi\right)+\frac{\sigma}{\varepsilon^{2}}\left(\phi_{\mathrm{e}}-\phi\right) \mathrm{d} \sigma\right]
$$

The model was applied to model the ring-shape Co ferrite composites. The model result has a sensitivity of $61 \mathrm{~A} / \mathrm{m}$ per $\mathrm{N} \bullet \mathrm{m}$ and a hysteresis $\pm 0.4 \mathrm{~N} \bullet \mathrm{m}$, which gave a prediction comparable with the experimental result.

\section{References:}

1. I. J. Garshelis, IEEE Trans. Magn., 28, 2202 (1992).

2. M.J. Sablik, D.C. Jiles, IEEE Trans. Magn., 35, 498 (1999).

3. Y. Chen, J. E. Snyder, C. R. Schwichtenberg, K. W. Dennis, R. W. McCallum, and D. C. Jiles, IEEE Trans. Magn.(in press).

4. D. C. Jiles, J. Phys. D: Appl. Phys., 281537 (1995). 


\section{REFERENCES}

1. J. P. Joule, Ann. Electr. Magn. Chem., 8, 219 (1842).

2. E. du T. de Lacheisserie, Magnetostriction: theory and applications of magnetoelasticity, CRC Press, Ann Arbor, 1993.

3. T. Kobayosi, Repts of the Aeronautical Inst. Of Tokyo Univ., 52, (1929).

4. A. E. Clark, in Ferromagnetic Materials, Vol. 1, edited by E. P. Wohlfarth, NorthHolland, Amsterdam, 1980, pp531-89.

5. R. Abachi, Sensors, p.82, April 1996.

6. V. Lemarquand, J. Magn. and Magn. Mater., 104-107, pt 2, 1109 (1992).

7. R. A. Beth, W. W. Meeks, Rev. Sci. Instr., 25, 603 (1954).

8. F.Koga and I. Sasada, IEEE Trans. Magn., 313, 143 (1995).

9. H. Hase, R. Shoji, US patent 5442966, 1994.

10. A. Hossain and M. Rashid, IEEE Trans. Magn., 26, 1158 (1990).

11. I. Sasada, A. Hiroike, K. Harada, IEEE Trans. Magn., MAG-20, 951 (1984).

12. K. Mohri, IEEE Trans. Magn., MAG-20, 924 (1984).

13. M. Liniers, V. madurga, M. Vazquez, A. Hernando, Phys. Rev. B, 31, 4425 (1985).

14. I. J. Garshelis, IEEE Trans. Magn., 28, 2202 (1992).

15. D. C. Jiles, Introduction to Magnetism and Magnetic Materials, Second Edition Chapman, \& Hall, New York, 1998.

16. R. M. Bozorth, Ferromagnetism, D. Van Nostrand Compnay, Inc. New York, 1978. 
17. S. Chikazumi, Physics of Magnetism, Chapter 8, John Wiley \& Sons Inc, New York, 1964.

18. R. D. Greenough and M.P. Schulze, Intermetallic Compounds: Vol. 2, Practice, Chapter 17, Edited by J.H. Westbrook and R.L. Fleischer, John Wiley \& Sons ltd., New York, 1994.

19. B. D. Cullity, Introduction to Magnetic Materials Addison Wesley, Reading, MA, 1972 p.268.

20. J. Smit, and H.P.J. Wijn, Ferrites, John Wiley \& Sons Inc., New York, 1959.

21. A. E. Clark, AIP Conf. Proc., No. 18, 1015 (1974).

22. A. E. Clark, H. S. Belson, and R.E. Strakna, J. Appl. Phys., 44, 2913 (1973).

23. A. E. Clark, T.P. O.D. McMasters, J. Appl. Phys., 63, 3910 (1988).

24. W. Mei, T. Okane, T. Umeda, and S. Zhou, J. Alloys and Compounds, 248, 151 (1997).

25. K.R.Dhilsha, G. Markandeyulu and K.V.S. Rama Rao, J. Appl. Phys., 70, 4450 (1991).

26. D.C.Jiles, and J.B. Thoelke, J. Magn. and Magn. Mater., 134. 143 (1994).

27. A. E. Clark and H. S. Belson, US Patent No. 4,378,258 (March 1983).

28. L Sandlund, M. Fahlander, T. Cedell, A. E. Clark, J. B. Restorff, and M. Wun-Fogle, J. Appl. Phys., 75, 5656 (1994).

29. F. E. Pinkerton, T. W. Capehart, J. F. Herbst, E.G. Brewer, and C.B. Murphy, Appl. Phys. Lett., 70, 2601 (1997).

30. A. Goldman, Modern Ferrite Technology, Van Nostrand Reinhold, New York ,1990 p215.

31. N. M. Gumen, Sov. Phys. J.E.T.P., 22, 251(1966). 
32. K.P. Belov, A.N. Goryaga, A.N. Lyamzin, Soviet Phys. Solid State, 27, 1101 (1985).

33. R. M. Bozorth, E.F. Tilden, A.J. Williams, Phys. Rev., 99, 1788 (1955).

34. C. Guillaud, Rev. Modern. Phys., 25, 64, (1953).

35. R. Valenzuela, Magnetic ceramics, Cambridge University Press, 1994.

36. V. Blaskov, V. Petkov, V. Rusanov, L.M. Martinez, B. Martinez, J.S. Munoz, M. Mikhov, J. Magn. and Magn. Mater., 162. 331 (1996).

37. C. S. Kim, Y.S. Yi, K. T. Park, H. Namgung, and J. G. Lee, J. Appl. Phys., 85, 5223 (1999).

38. D. C. Jiles, J. Phys. D: Appl. Phys., 281537 (1995).

39. R. R. Birss, C.A. Faunce, and E. D. Isaac, J. Phys. D: Appl. Phys., 4, 1040 (1971).

40. M. Arib, J.L. Ille, Int. J. Appl. Electromagn. and Mech., 8, 329 (1997).

41. M.K. Devine, D.C. Jiles, J. Appl. Phys., 79, 5493 (1996).

42. J.M. Makar and D.L. Atherton, IEEE Trans. Magn., 31, 877 (1995).

43. M.G. Maylin, and P. T. Squire, IEEE Trans. Magn., 29, 3449 (1993).

44. M. Roberston, IEEE Trans. Magn., 29, 2077 (1993).

45. D.C. Jiles, and D.L. Atherton, J. Phys. D: Appl. Phys., 17, 1265 (1984).

46. D.J. Craik, and M.J. Wood, J. Phys. D: Appl. Phys., 4, 1009 (1971).

47. I. J. Garshelis, and C.A. Jones, J. Appl. Phys., 85, 5468 (1999).

48. W. J. Fleming, IEEE Trans. Vehi. Tech., 38, 159 (1989).

49. M.J. Sablik, D.C. Jiles, IEEE Trans. Magn., 35, 498 (1999).

50. M.J. Sablik, D.C. Jiles, J. Phys. D: Appl. Phys., 32, 1971 (1999).

51. W. J. Fleming, Society of Automotive Engineers, 890482 and 890483, 1989. 
52. I. J. Garshelis, IEEE Trans. Magn., 29, 3201 (1993).

53. I. J. Garshelis, and C. R. Conto, IEEE Trans. Magn., 30, 4629 (1994).

54. I. J. Garshelis, IEEE Trans. Magn., 32, 4812 (1996).

55. V. Masheva, M. Grigorova, D. Nihtianova, J.E. Schmidt, and M. Mikhov, J. Phys. D: Appl. Phys., 32, 1595 (1999).

56. Y. Chen, J. E. Snyder, C. R. Schwichtenberg, K. W. Dennis, D. K. Falzgraf, R. W. McCallum, and D. C. Jiles, Appl. Phys. Lett., 74,1159 (1999).

57. C.W. Allen, and W.H. Kao, Technical report (THEMIS-UND-70-11) prepared by the Magnetomechanical group at the University of Notre Dame, 1970.

58. Y. Chen, J. E. Snyder, C. R. Schwichtenberg, K. W. Dennis, R. W. McCallum, and D. C. Jiles, paper EF-06, Intermag'99, Kyongju, Korea, May 1999, to be published in IEEE Trans. Magn., Nov., 1999.

59. J. G. Lee, J Y. Park, and C. S. Kim, J. Mater. Sci., 33, 3965 (1998).

60. S.I. Hirano, J.I. Watanabe, and S. Naka, Advances in Ceramics, ICF4, 15, 65 (1984).

61. P.P. Pulvirenti, D.C. Jiles, R. D. Greenough, and I.M. Reed, J. Appl. Phys., 79, 6219 (1996).

62. I. J. Garshelis, IEEE Trans. Magn., 2 , 344 (1974).

63. P.E. Tannenwald, Phys. Rev., 99, 463 (1955).

64. G.E. Dieter, Mechanical Metallurgy, SI Metric Edition, McGraw-Hill Book Company, New York, 1988.

65. F. Preisach, Z. Phys., 94, 277 (1935).

66. E.C. Stoner and E.P. Wohlforth, Phil. Trans. Roy. Soc., A240, 599 (1948). 
67. D. C. Jiles and D.L. Atherton, J. Magn. And Magn. Mater., 61, 48 (1986).

68. M.J. Sablik, and D. C. Jiles, IEEE Trans. Magn., 29 , 2113 (1993).

69. P.A. Voltairas, D.I. Fotiadis and C.V. Massalas, J. Magn. And Magn. Mater. 204. 135 (1999).

70. J. F. Herbst, T. W. Capehart, F. E. Pinkerton, Appl. Phys. Lett., 70, 3041 (1997).

71. W. D. Callister, "Materials Science and Engineering: An Introduction", 4th edition, John Wiley \& Sons Inc., New York, 1997, p513.

72. C.W. Nan, Appl. Phys. Lett., 72, 2897 (1998).

73. G. W. Kaye, T. H. Laby, Tables of Physical and Chemical Constants, John Wiley \& Sonc Inc., New York, 1995.

74. A.E. Clark, M. Wun-Fogle, J. P. Teter, J. B. Restorff, S.F. Cheng, J. Appl. Phys., 76, 7009 (1994).

75. M.J. Sablik, S.W. Rubin, L.A. Riley, D.C. Jiles, D.A. Kaminski, and S. B. Biner, J. Appl. Phys., 74, 480 (1993).

76. M.J. Sablik, G.L. Burkhardt, H. Kwun, and D.C. Jiles, J. Appl. Phys., 63, 3930 (1988).

77. D.A. Kaminski, D.C. Jiles, and M.J. Sablik, J. Magn. and Magn. Mater., 140-144, 382 (1992).

78. J.F. Herbst, F.E. Pinkerton, J. Magn. and Magn. Mater., 176, 183 (1997).

79. W. D. Armstrong, J. Appl. Phys., 81, 2321 (1997).

80. İ. J. Garshelis and C.R. Conto, J. Appl. Phys., 79, 4756 (1996).

81. D.C. Jiles, J.B. Thoelke, M.K. Devine, IEEE Trans. Magn., 28, 27 (1992). 


\section{ACKNOWLEDGEMENTS}

This work was supported by the US Department of Energy, Office of Energy Research, Office of Computational and Technology Research, Advanced Energy Projects Division. Ames Laboratory is operated by the U.S. Department of Energy by Iowa State University under contract W-7405-ENG-82. The United States government has assigned the DOE Report number IS-T 1899 to this thesis.

First, I would like to express my sincere appreciation to my major professor Dr. David C. Jiles for his supervision, encouragement, support and advice throughout my study and life in the last two years. The influence of his professional guidance was far beyond this dissertation and will be beneficial to my future. Also, I would like to thank Dr. Miller, Dr. Otaigbe, Dr. Trivedi, Dr. Udpa and Dr. Weber for dedicating time to serve on the committee of my program of study and for their patience.

I would also like to thank Dr. J. E. Snyder, Dr. M. J. Sablik, Dr. R. W. McCallum, Dr. K. W. Dennis, Dr. C. C. H. Lo, Dr. M. J. Johnson, and Dr. Y. Bi, for their helpful support and suggestion. Special thanks to all members of Magnetics Group, Ames Laboratory.

I would like to dedicate this dissertation to my grandmother and my parents for their guidance, love, and sacrifice in the course of my life. Finally, a hearty thanks goes to my wife, Ruoqi, for her inspiration, encouragement, and support. She made my study and life at Iowa State University enjoyable and productive and a wonderful memory. 